\title{
Hydrophobic Pockets of HPMC Enable Extremely Short Reaction Times in Water
}

Desislava Petkova, ${ }^{a}$ Niginia Borlinghaus, ${ }^{a}$ Sudripet Sharma, ${ }^{b}$ Johannes Kaschel,,${ }^{a}$ Tanja Lindner, ${ }^{a}$ Johanna Klee, ${ }^{a}$ Anais Jolit, ${ }^{a}$ Vanessa Haller, ${ }^{\text {a }}$ Stephanie Heitz, ${ }^{a}$ Katarina Britze, ${ }^{a}$ Justin Dietrich, ${ }^{c}$ Wilfried M. Braje, ${ }^{a *}$ Sachin Handa*b

${ }^{a}$ Neuroscience Discovery Research, AbbVie Deutschland GmbH \& Co. KG, Knollstraße, 67061 Ludwigshafen, Germany

${ }^{b}$ Department of Chemistry, University of Louisville, 2320 S Brook St. Louisville, KY 40292, USA

${ }^{\mathrm{c}}$ Research and Development, AbbVie Inc, 1400 Sheridan Rd, North Chicago, Illinois, USA

*Correspondence Authors: wilfried.braje@abbvie.com; sachin.handa@louisville.edu

Supporting information includes:

Total number of pages: 113

Total number of figures: 18

Total number of tables: 4

1. General Experimental Details $\quad$ S2

2. Preparation of Aqueous HPMC Solutions $\quad$ S3

3. General Procedures for Buchwald-Hartwig Amination Reactions S4

4. Optimization of Buchwald-Hartwig Aminations for Scale-up S5-S6

5. Characterization of Active Catalyst S6-11

6. Control Reactions in Water for Buchwald-Hartwig Aminations S12-S13

7. Synthesis and Characterization of Amination Products S14-31

8. General Procedure for Amide Couplings S31-33

9. Amide Couplings: Optimizations Studies S34-38

10. Synthesis and Characterization of Amide Coupling Products S39-51

11. Control Reactions in Water for Amide Couplings S52

12. Synthesis and Characterization of Four Step One-Pot Sequence S53-56

13. Spectral Data of Buchwald-Hartwig Amination Products S57-91

14. Spectral Data of Amide Coupling Products S92-110

15. Spectral Data Four Step One-Pot Sequence S1110-112

$\begin{array}{ll}\text { 16. References } & \text { S113 }\end{array}$ 


\section{General Experimental Details}

All reactions were carried out in microwave vials from Biotage. All reagents were purchased from commercial suppliers and used without further purification. Concentrating the organic solvent was performed under reduced pressure on a Büchi Rotavapor R-100 with heating bath B-100.

The progress of the reactions was monitored by LC-MS Agilent 1200 series instruments controlled by Agilent ChemStation Software, GC-MS using a Thermo Scientific Trace 1300 Gas Chromatograph coupled with a Thermo Scientific ISQ-QD Single Quadrupole, and/or thin-layer chromatography (TLC) on Silica gel 60 F254 aluminium plates (Merck). Chromatograms were visualized by UV light at $254 \mathrm{~nm}$. Telos bulk sorbent (diatomaceous earth) was purchased from Kinesis.

Automated flash column chromatography was performed on CombiFlash $\mathrm{R}_{\mathrm{f}}+$ Teledyne Isco, using High Performance RediSepR $R_{f}$ Gold silica gel columns (4 Gram Flash Column; CV $4.8 \mathrm{~mL}-18 \mathrm{~mL} / \mathrm{min}$; max. pressure 600 psi (41 bar); $20 \mathrm{mg}-400 \mathrm{mg}$ sample).

Preparative SFC separations were carried out on a Waters Prep 100q SFC System, controlled by Waters MassLynx Software. The columns were $150 \mathrm{~mm}$ in length, $30 \mathrm{~mm}$ in diameter and packed with $5 \mu$ m material. A mixture of liquefied $\mathrm{CO}_{2}$ and methanol $+0.2 \%$ $\mathrm{NH}_{4} \mathrm{OH}$ was used as mobile phase.

${ }^{1} \mathrm{H}$ and ${ }^{13} \mathrm{C}$ NMR spectra were recorded at $23{ }^{\circ} \mathrm{C}$ on Bruker AV-600 or AV-500 or Varian MR-400 or Varian Unity INOVA 500 spectrometers. Chemical shifts $(\delta)$ values are presented in parts per million (ppm), referenced to the solvent peak $\left(\mathrm{CDCl}_{3}\right.$, defined at $\delta 7.26$ ppm for ${ }^{1} \mathrm{H}, \delta 77.0 \mathrm{ppm}$ for ${ }^{13} \mathrm{C}$; $\mathrm{CD}_{3} \mathrm{OD}: \delta 3.31 \mathrm{ppm}$ for ${ }^{1} \mathrm{H}, \delta 49.0 \mathrm{ppm}$ for ${ }^{13} \mathrm{C}$; $\left(\mathrm{CD}_{3}\right)_{2} \mathrm{SO}: \delta 2.50 \mathrm{ppm}$ for ${ }^{1} \mathrm{H}, \delta 39.5 \mathrm{ppm}$ for ${ }^{13} \mathrm{C} .{ }^{19} \mathrm{~F}$ chemical shifts are reported in ppm relative to $\mathrm{CFCl}_{3}(0 \mathrm{ppm})$. Data are reported as follows: chemical shift $(\delta)$, multiplicity $(\mathrm{s}=$ singlet, $\mathrm{d}=$ doublet, $\mathrm{q}=$ quartet, quint = quintet, $\mathrm{m}=$ multiplet, $\mathrm{bs}=$ broad singlet, $\mathrm{bd}=$ broad doublet, $\mathrm{dd}=$ doublet of doublet, $\mathrm{dt}=$ doublet of triplets $)$, coupling constants $(\mathrm{J}, \mathrm{Hz})$ and integration.

High resolution mass spectra were obtained on a Bruker Daltonics micro TOF-Q. All masses are given in atomic units/elementary charge $(\mathrm{m} / \mathrm{z})$ and reported in percentage relative to the basic peak.

Infrared spectra were recorded with a Perkin-Elmer Spectrum 100 FT-IR spectrometer. 


\section{Preparation of Aqueous HPMC Solutions}

In order to have reproducible conditions and exclude any influence of traces of metals or metal ions present in common distilled water, Milli- $Q^{\circledR}$ water was used. This 'ultrapure' water was obtained with an EMD Millipore Milli-Q ${ }^{\mathrm{TM}}$ Advantage A10 water purification system from EMD Millipore Z00Q0V0US. Herein this water is termed 'Millipore water'. For the preparation of the aq. HPMC (hydroxypropylmethyl cellulose) solutions commercially available Mantrocel ${ }^{\circledR}$ E5 HPMC (4-6 cps $\left.=\mathrm{mPa} \cdot \mathrm{s}\right)$ was used. In the present study Mantroce ${ }^{\circledR}$ E5 HPMC from Gustav Parmentier GmbH, Frankfurt, Germany was used. We would like to thank Gustav Parmentier GmbH for this generous gift.

\subsection{Procedure for the preparation of a $2 \mathrm{wt} \% \mathrm{HPMC}$ water solution (per $100 \mathrm{~mL}$ ):}

$66 \mathrm{~mL}$ Millipore water was heated to $70^{\circ} \mathrm{C}$ under stirring in a reaction flask. $2 \mathrm{~g}$ of HPMC were added. The mixture formed a cloudy solution. Subsequently $34 \mathrm{~mL}$ of Millipore water were added, and the mixture was allowed to cool to room temperature under stirring to form a clear solution. The solution was purged with Argon for $30 \mathrm{~min}$.

\subsection{Procedure for the preparation of a $0.5 \mathrm{wt} \% \mathrm{HPMC} /$ water solution (per $100 \mathrm{~mL}$ ):}

$66 \mathrm{~mL}$ Millipore water was heated to $70{ }^{\circ} \mathrm{C}$ under stirring in a reaction flask. $500 \mathrm{mg}$ of HPMC were added. The mixture formed a cloudy solution. Subsequently $34 \mathrm{~mL}$ of Millipore water were added, and the mixture was allowed to cool to room temperature under stirring to form a clear solution. The solution was purged with Argon for $30 \mathrm{~min}$.

\subsection{Procedure for the preparation of a $0.1 \mathrm{wt} \% \mathrm{HPMC} /$ water solution (per $100 \mathrm{~mL}$ ):}

$66 \mathrm{~mL}$ Millipore water was heated to $70{ }^{\circ} \mathrm{C}$ under stirring in a reaction flask. $100 \mathrm{mg}$ of HPMC were added. The mixture formed a cloudy solution. Subsequently $34 \mathrm{~mL}$ of Millipore water were added, and the mixture was allowed to cool to room temperature under stirring to form a clear solution. The solution was purged with Argon for $30 \mathrm{~min}$. 


\section{General Procedures for Buchwald-Hartwig Amination Reactions}

\subsection{General procedure for Buchwald-Hartwig amination reactions (I)}

Under an inert atmosphere (glovebox), sodium tert-butoxide (1.5 equiv.), bis[cinnamyl palladium(II)chloride] (1.1 mol\%), 2-di-tert-butylphosphino-2', 4',6'triisopropylbiphenyl (4.4 mol\%), aryl bromide (1.0 equiv., $0.5 \mathrm{mmol})$, and the corresponding amine/amide (1.25 equiv.) were added into a $5 \mathrm{~mL}$ microwave vial containing a PTFE-coated magnetic stir bar and a rubber septum. 1.5 mL of HPMC solution ( $2 \mathrm{wt} \%$ Mantrocel E5 in degassed Millipore water) was added and the reaction mixture was stirred at $50{ }^{\circ} \mathrm{C}$ with $1200 \mathrm{rpm}$ for the indicated time. Ethyl acetate and sat. aq. $\mathrm{Na}_{2} \mathrm{SO}_{4}$ solution (same amount as HPMC/water solution) were added to the reaction mixture and the mixture was transferred to a separatory funnel. The microwave vial was washed a few times with EtOAc and water and contents were transferred to the separatory funnel. The aq. phase was washed 3x with EtOAc. Note: If an emulsion is forming during the extraction (originating from crashed out HPMC), extensive extraction with EtOAc is needed in order to obtain high yields. The combined organic layers were concentrated in vacuo and the residue was further purified by automated flash column chromatography (silica gel, gradient $0-100 \%$ ethyl acetate/heptane).

\subsection{General procedure for Buchwald-Hartwig amination reactions (II)}

Under an inert atmosphere (glovebox), sodium tert-butoxide (1.5 equiv.), bis[cinnamyl palladium(II)chloride $] \quad(1.1 \quad$ mol\%), 2-di-tert-butylphosphino-2', 4',6'triisopropylbiphenyl (4.4 mol\%), aryl bromide (1.0 equiv., $0.5 \mathrm{mmol})$, and the corresponding amine/amide (1.25 equiv.) were added into a $5 \mathrm{~mL}$ microwave vial containing a PTFE-coated magnetic stir bar and a rubber septum. HMPC solution (2 wt\% Mantrocel E5 in degassed Millipore water) was added and the reaction mixture was stirred at $50{ }^{\circ} \mathrm{C}$ with $1200 \mathrm{rpm}$ for the indicated time. $1 \mathrm{~mL} \mathrm{CH}_{2} \mathrm{Cl}_{2}$ was added to the reaction mixture and the mixture was transferred to a round bottom flask. The microwave vial was washed $3 \mathrm{x} \mathrm{CH}_{2} \mathrm{Cl}_{2}$ (in total ca. $15 \mathrm{~mL}$ ). Telos bulk sorbent was added, and the mixture was concentrated in vacuo. After drying an automated flash column chromatography was performed (silica gel, gradient $0-100 \%$ ethyl acetate/heptane). 


\section{Optimization of Buchwald-Hartwig Aminations for Scale-up}

Optimization of the reaction conditions was performed for the purpose of utilizing this transformation on larger scale (up to grams). The experiments shown in Table S1 were performed on $0.5 \mathrm{mmol}$ scale following general procedure (I).

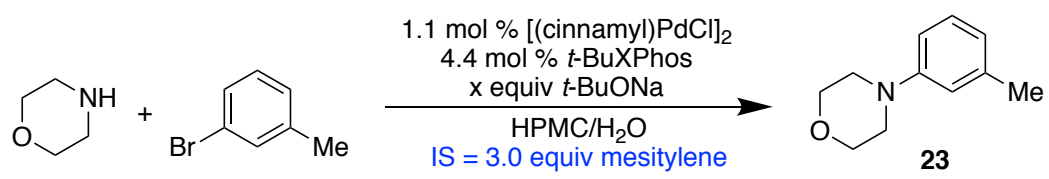

\begin{tabular}{llllccc}
\hline Entry $^{\mathbf{a}}$ & $\begin{array}{l}\text { Morpholine } \\
\text { [equiv.] }\end{array}$ & $\begin{array}{l}\mathbf{N a O}^{t} \mathbf{B u} \\
\text { [equiv.] }\end{array}$ & $\begin{array}{l}\mathbf{T} \\
{\left[{ }^{\circ} \mathbf{C}\right]}\end{array}$ & $\begin{array}{c}\text { time } \\
{[\mathbf{m i n}]}\end{array}$ & $\begin{array}{c}\text { HPMC } \\
{[\mathbf{w t} \%]}\end{array}$ & $\begin{array}{c}\text { IS yield }^{\mathbf{b}} \\
{[\%]}\end{array}$ \\
\hline 1 & 1.25 & 1.5 & 50 & 5 & 2 & quantitative, $91^{\mathrm{c}}$ \\
2 & 1.25 & 1.5 & $\mathrm{rt}$ & 30 & 2 & quantitative \\
3 & 1.0 & 1.5 & 50 & 5 & 2 & quantitative \\
4 & 1.0 & 1.0 & 50 & 5 & 2 & quantitative \\
5 & 1.0 & 1.0 & 50 & 10 & 0.1 & quantitative \\
$6^{\text {d }}$ & 1.0 & 1.0 & 50 & 10 & 0.1 & quantitative, 83 \\
7 & 1.0 & $1.0^{\mathrm{e}}$ & 50 & 10 & 0.1 & quantitative, $92^{\mathrm{c}}$ \\
\hline
\end{tabular}

Table S1. Optimization of reaction conditions for scale up experiment. ${ }^{\text {a }}$ Reactions were performed on $0.5 \mathrm{mmol}$ scale at $2.5 \mathrm{M}$ concentration following the general procedure (II) for Buchwald-Hartwig aminations. ${ }^{b}$ IS: internal standard yield determined using 0.3 equiv. mesitylene; ${ }^{\mathrm{c}}$ isolated yield; ${ }^{\mathrm{d}}$ Reaction was performed on $3 \mathrm{~g}(17.4 \mathrm{mmol}) \mathrm{scale} .{ }^{\mathrm{e}} 1.0 \mathrm{KOH}$ and 2.0 equiv. ${ }^{t} \mathrm{BuOH}$ was used instead of $\mathrm{NaO}^{t} \mathrm{Bu}$.

\subsection{Multigram synthetic procedure for 9,33 , and 34}

Under argon atmosphere, $\mathrm{KOH}$

palladium(II)chloride] (1.1 mol\%),

(1.5 equiv.), bis[cinnamyl 2-di-tert-butylphosphino-2', 4',6'triisopropylbiphenyl (4.4 mol\%), aryl bromide (1.0 equiv., $0.5 \mathrm{mmol}$ ), the corresponding amine/amide (1.25 equiv.), and ${ }^{t} \mathrm{BuOH}$ (2.0 equiv.) were added into a $100-200 \mathrm{~mL}$ roundbottomed flask containing a PTFE-coated magnetic stir bar and a rubber septum. Aqueous HMPC solution (0.2 wt\% Mantrocel E5 in degassed Millipore water) was added (1 M) and the reaction mixture was stirred at $50{ }^{\circ} \mathrm{C}$ with $1200 \mathrm{rpm}$ for the indicated time. $40 \mathrm{~mL}$ $\mathrm{CH}_{2} \mathrm{Cl}_{2}$ was added to the reaction mixture and the mixture was stirred for 5 minutes. Aqueous layer was separated and further washed with $(2 \times 10 \mathrm{~mL}) \mathrm{CH}_{2} \mathrm{Cl}_{2}$. Combined 
organic layers were dried over anhydrous $\mathrm{MgSO}_{4}$. Volatiles were removed under reduced pressure to obtain crude product. Crude product was purified by automated flash column chromatography over silica gel, gradient $0-100 \%$ ethyl acetate/heptane.

\section{Characterization of Active Catalyst}

\subsection{HRTEM analysis.}

Sample preparation: Under argon atmosphere, bis[cinnamyl palladium(II)chloride] ( $2.9 \mathrm{mg}, 1.1 \mathrm{~mol} \%$ calculated for $0.5 \mathrm{mmol} \mathrm{scale} \mathrm{catalytic} \mathrm{reaction),}$ 2-di-tert-butylphosphino-2',4',6'-triisopropylbiphenyl (9.3 mg, $4.4 \mathrm{~mol} \%$ calculated for $0.5 \mathrm{mmol}$ scale catalytic reaction), and sodium tert-butoxide $(72 \mathrm{mg}$ ) or $\mathrm{KOH}(42 \mathrm{mg}$ ), were added to $4 \mathrm{~mL}$ reaction vial containing PTFE-coated magnetic stir bar. Reaction vial was closed with a rubber septum. Reaction mixture was evacuated and backfilled with argon. $1 \mathrm{~mL}$ of aqueous solution of HMPC ( $0.2 \mathrm{wt} \%$ Mantrocel E5 in degassed Millipore water) was added and the reaction mixture was stirred at $50{ }^{\circ} \mathrm{C}$ with $1200 \mathrm{rpm}$ for 5 minutes. Reaction mixture was cooled to rt and immediately analyzed for analysis.

HRTEM analysis were conducted using a $200-\mathrm{kV}$ field emission gun FEI Tecnai F20 microscope and specimens prepare by drop casting catalyst dispersions onto commercial copper grid-supported amorphous holey carbon films.
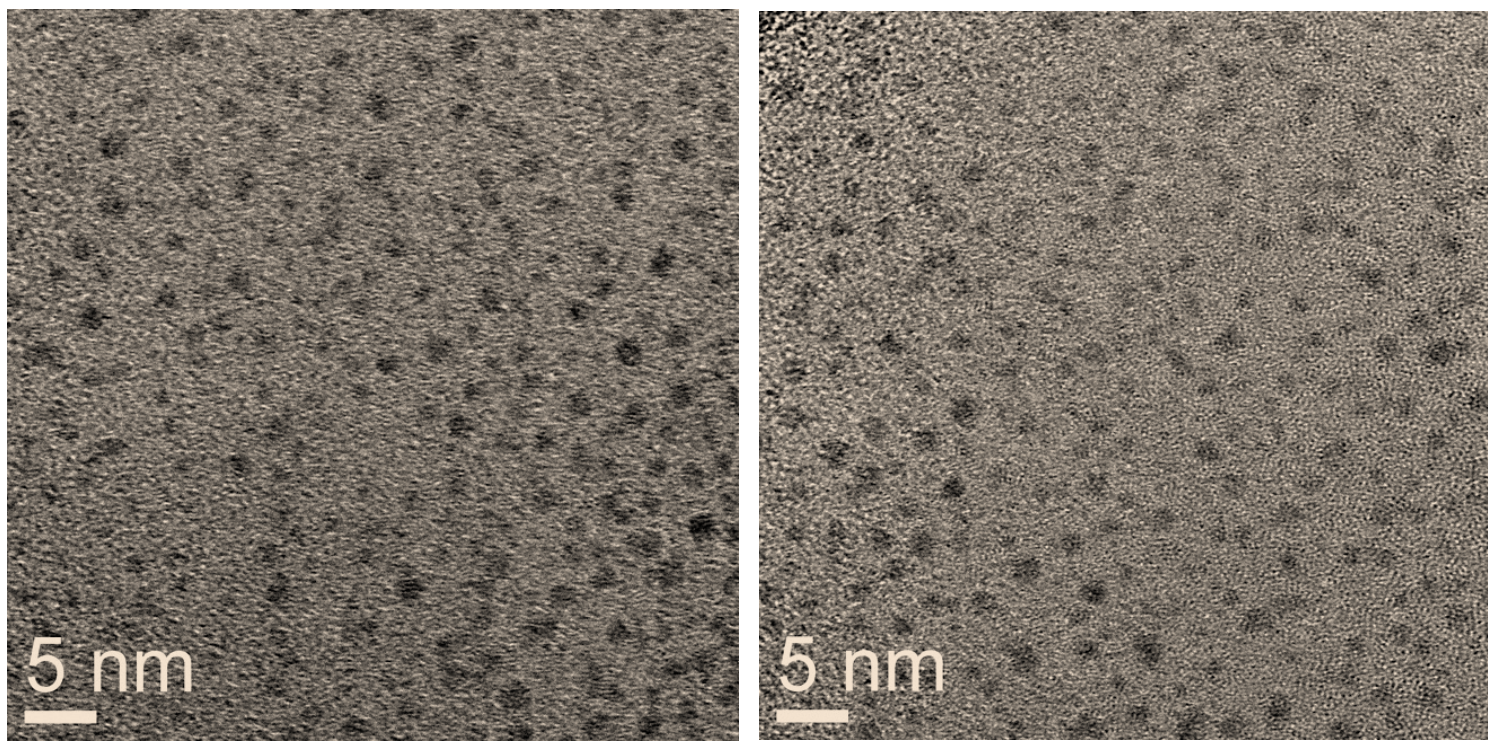


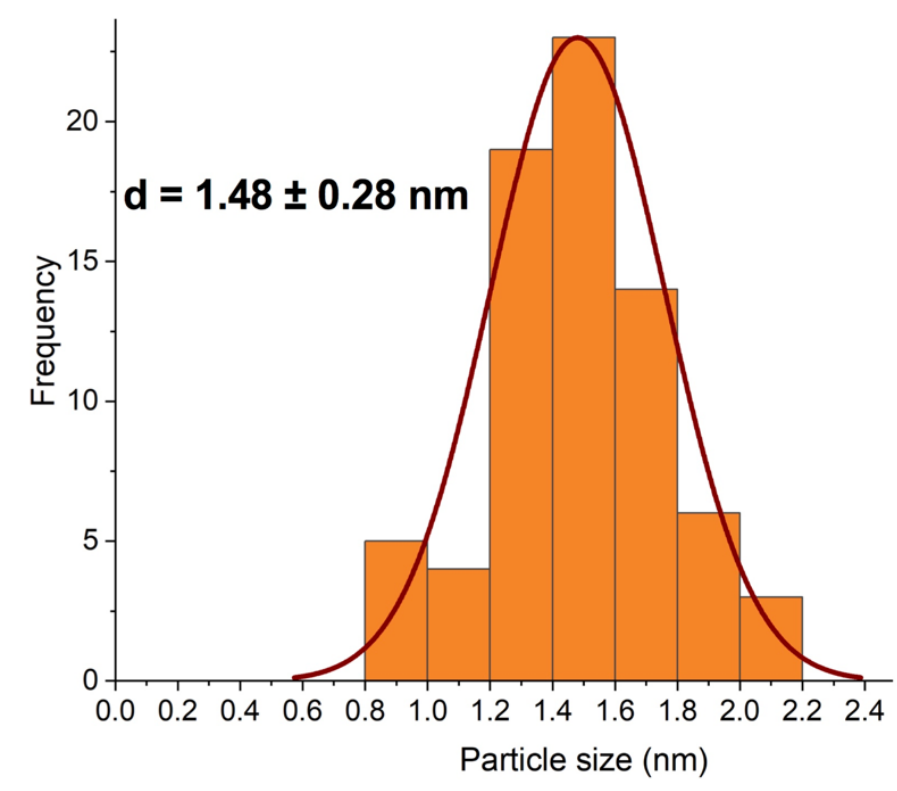

Figure S1. HRTEM analysis of in-situ formed palladium nanoparticles.

\subsection{EDAX analysis of the catalyst}

The aqueous solution containing palladium nanoparticles was also analyzed for EDAX analysis. Analysis and mapping confirmed the presence of phosphorus from $t$-BuXPhos, oxygen atoms from HPMC, palladium from the nanocatalyst. The relative atomic concentrations of $\mathrm{P}, \mathrm{O}$, and $\mathrm{Pd}$, estimated based on EDAX spectra are $12 \%, 28 \%$, and $4 \%$ respectively. On the other hand, the omission of $\mathrm{C}$ from the elemental quantification was due to the issue of the overlap between Pd M zeta and C K alpha. The Pd M zeta line is at $282 \mathrm{eV}$ while the $\mathrm{C} \mathrm{K}$ alpha is at $277 \mathrm{eV}$, giving a separation of $4 \mathrm{eV}$ only, which is significantly below the energy resolution of EDAX. 

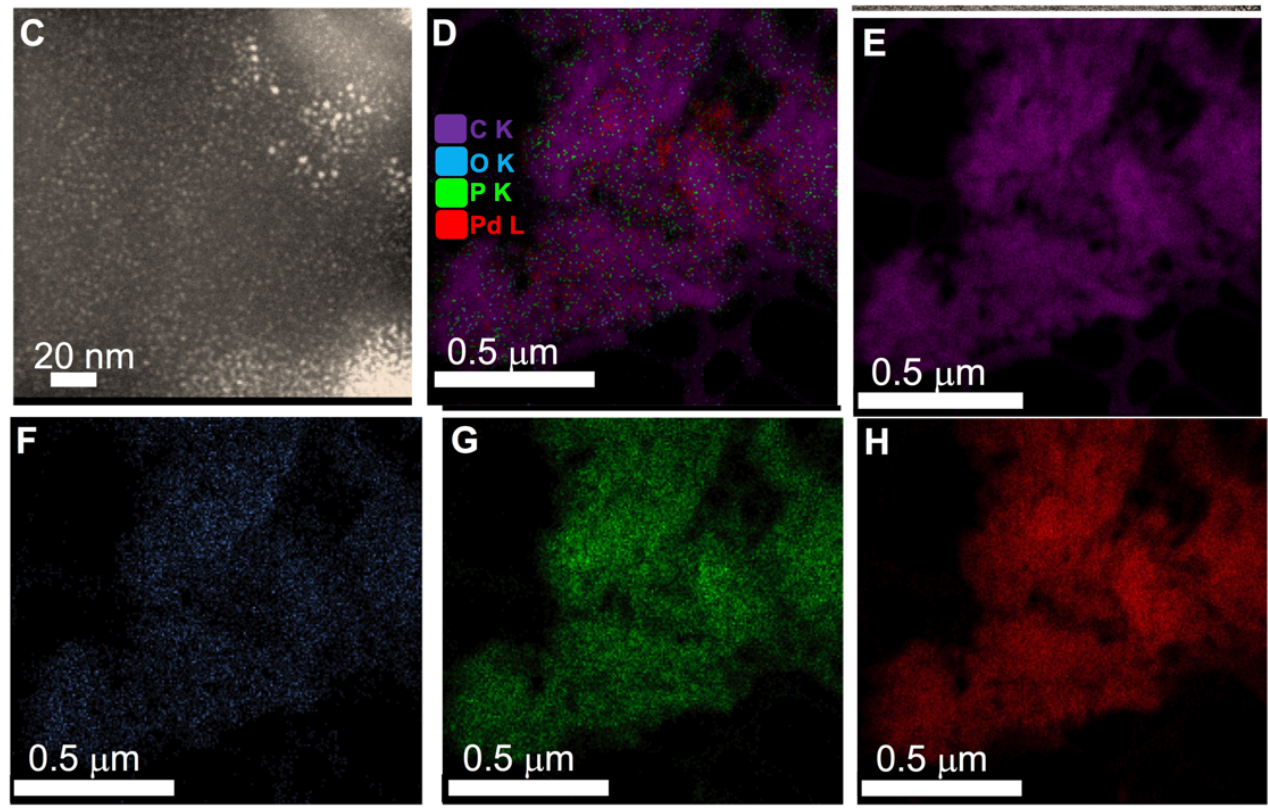

$0.5 \mu \mathrm{m}$

$0.5 \mu \mathrm{m}$

$0.5 \mu \mathrm{m}$

eZAF Smart Quant Results

\begin{tabular}{ccc}
\hline element & atomic \% & error \% \\
\hline O K & 28.36 & 6.47 \\
P K & 9.30 & 7.23 \\
Pd L & 4.07 & 4.62 \\
\hline
\end{tabular}

Figure S2. EDAX analysis of nanoparticles.

\subsection{Catalytic reaction with nanoparticles}<smiles>Brc1cccc2ccccc12</smiles><smiles>Cc1ccc(N)cc1</smiles>

\section{nanoparticles} containing base<smiles>Cc1ccc(Nc2cccc3ccccc23)cc1</smiles>

Only $0.5 \mathrm{~mL}$ sample was consumed for HRTEM and EDAX analysis. The remaining half solution was explored for catalytic activity.

To the aqueous solution containing palladium nanoparticles and base, 1-bromonaphthalene $(0.25 \mathrm{mmol})$ and $p$-toluidine $(0.26 \mathrm{mmol})$ were added under argon atmosphere. Reaction mixture was stirred at $50{ }^{\circ} \mathrm{C}$ for 5 minutes. After 5 minutes, stirring was stopped and 
reaction mixture was cooled to rt. $2 \mathrm{~mL} \mathrm{CH}_{2} \mathrm{Cl}_{2}$ was added to the mixture and mixture was stirred for a minute. Organic layer was separated from the aqueous and then dried over anhydrous $\mathrm{MgSO}_{4}$. Volatiles were removed under reduced pressure to obtain crude product, which was purified by flash chromatography using EtOAc/hexanes (1:9, 1:4). Pure product was obtained as colorless oil, $93 \%$ yield (55 mg).

\subsection{Analysis by ${ }^{31}$ P NMR spectroscopy}

The nanoparticles were suspended in $0.2 \mathrm{wt} \% \mathrm{HPMC}$ in $\mathrm{D}_{2} \mathrm{O}$ were prepared according to the procedure provided in section 9.1. $0.15 \mathrm{~mL}$ aliquot withdrawn from the nanoparticle stock solution was dissolved in $0.30 \mathrm{~mL} \mathrm{DMSO}-\mathrm{d}_{6}$ and was analyzed with ${ }^{31} \mathrm{P} \mathrm{NMR}$ spectroscopy using Varian Innova $400 \mathrm{MHz}$ NMR spectrophotometer. (Note: The internal standard was prepared in a capillary tube by dissolving $P P_{3}$ in $D M S O\left(d_{6}\right)$, and tip of capillary tube was permanently sealed).

\begin{tabular}{|c|c|}
\hline Sample ID & Constituent \\
\hline Sample 1 & $t$-BuXPhos in DMSO- $\mathrm{d}_{6}(0.03 \mathrm{M})$ \\
\hline Sample 2 & $\begin{array}{c}t \text {-BuXPhos }+ \text { bis }[\text { cinnamyl palladium }(\mathrm{II}) \text { chloride }](4: 1) \text { in } \\
\text { mL of DMSO- } \mathrm{d}_{6}(0.03 \mathrm{M})\end{array}$ \\
\hline Sample 3 & $\begin{array}{l}t \text {-BuXPhos }+ \text { bis[cinnamyl palladium(II)chloride] }(2: 1) \text { in } \\
\text { mL of DMSO- } \mathrm{d}_{6}(0.05 \mathrm{M})\end{array}$ \\
\hline Sample 4 & Analyte (nanoparticles) in $\mathrm{D}_{2} \mathrm{O} / \mathrm{DMSO}-\mathrm{d}_{6}(1: 2,0.02 \mathrm{M})$ \\
\hline
\end{tabular}




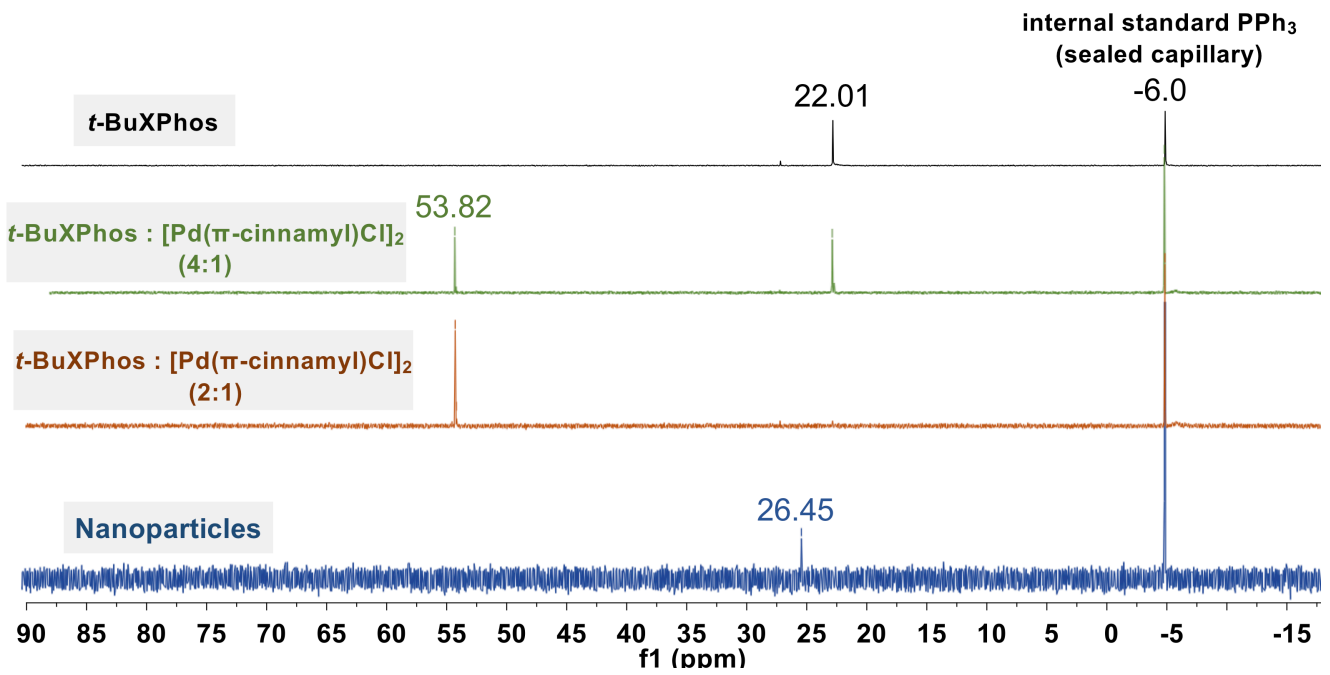

Figure S3. ${ }^{31} \mathrm{P}$ NMR spectra of NPs (bottom) compared to $t$-BuXPhos and organometallic molecular complex $\left(\mathrm{PPh}_{3}\right.$ as internal std).

\subsection{Catalytic activity of fresh and aged catalyst}

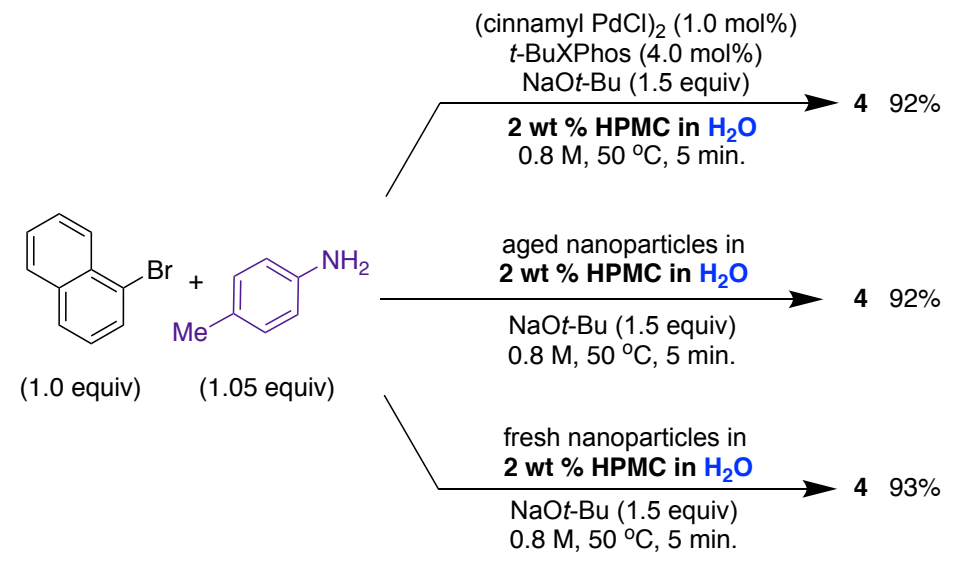

Catalytic reaction with fresh nanoparticles: Under argon atmosphere, bis[cinnamyl palladium(II)chloride] $\quad(2.8 \mathrm{mg}, \quad 1$ mol\%), 2-di-tert-butylphosphino-2',4',6'triisopropylbiphenyl (9 mg, $4.0 \mathrm{~mol} \%$ ), and sodium tert-butoxide (72 mg) was added to the $4 \mathrm{~mL}$ microwave reaction vial containing PTFE-coated magnetic stir bar. Reaction vial was closed with a rubber septum. Reaction vial was closed and backfilled with argon. To the reaction vial, $1 \mathrm{~mL}$ of aqueous solution of HMPC (2 wt\% Mantrocel E5 in degassed Millipore water) was added and the reaction mixture was stirred at $50{ }^{\circ} \mathrm{C}$ with $1200 \mathrm{rpm}$ for 5 minutes which generates the active nanoparticle catalyst. To the catalyst solution, 1bromonaphthalene $(0.5 \mathrm{mmol})$ and $p$-toluidine $(0.52 \mathrm{mmol})$ were added and mixture was 
heated at $50^{\circ} \mathrm{C}$ for five minutes. After 5 minutes, stirring was stopped and reaction mixture was cooled to rt. $3 \mathrm{~mL} \mathrm{CH}_{2} \mathrm{Cl}_{2}$ was added to the mixture and mixture was stirred at rt for a minute. Organic layer was separated from the aqueous and then dried over anhydrous $\mathrm{MgSO}_{4}$. Volatiles were removed under reduced pressure to obtain crude product, which was purified by flash chromatography using EtOAc/hexanes $(1: 9,1: 4)$. Pure product was obtained as colorless oil, 93\% yield (110 mg).

Catalytic reaction with aged nanoparticles. Under argon atmosphere, bis[cinnamyl palladium(II)chloride] $\quad(2.8 \mathrm{mg}, \quad 1$ mol\%), 2-di-tert-butylphosphino-2',4',6'triisopropylbiphenyl ( $9 \mathrm{mg}, 4.0 \mathrm{~mol} \%$ ), and sodium tert-butoxide (72 $\mathrm{mg}$ ) was added to the $4 \mathrm{~mL}$ microwave reaction vial containing PTFE-coated magnetic stir bar. Reaction vial was closed with a rubber septum. Reaction vial was closed and backfilled with argon. To the reaction vial, $1 \mathrm{~mL}$ of aqueous solution of HMPC ( $2 \mathrm{wt} \%$ Mantrocel E5 in degassed Millipore water) was added and the reaction mixture was stirred at $50{ }^{\circ} \mathrm{C}$ with $1200 \mathrm{rpm}$ for 5 minutes which generates the active nanoparticle catalyst. These nanoparticles were stored at $\mathrm{rt}$ for next 6 hours.

After $6 \mathrm{~h}, 1$-bromonaphthalene $(0.5 \mathrm{mmol})$ and $p$-toluidine $(0.52 \mathrm{mmol})$ were added to the vial containing above nanoparticle solution. Under argon atmosphere, mixture was heated at $50{ }^{\circ} \mathrm{C}$ for five minutes. After 5 minutes, stirring was stopped and reaction mixture was cooled to rt. $3 \mathrm{~mL} \mathrm{CH}_{2} \mathrm{Cl}_{2}$ was added to the mixture and mixture was stirred at $\mathrm{rt}$ for a minute. Organic layer was separated from the aqueous and then dried over anhydrous $\mathrm{MgSO}_{4}$. Volatiles were removed under reduced pressure to obtain crude product, which was purified by flash chromatography using EtOAc/hexanes (1:9, 1:4). Pure product was obtained as colorless oil, 92\% yield (108 mg).

Catalytic reaction with in-situ generated nanoparticles. Standard general procedure was used for this reaction. Pure product was obtained after standard purification procedure as a colorless oil, 92\% yield (108 mg). 


\section{Control Reactions in Water for Buchwald-Hartwig Aminations}

In order to validate whether HPMC has a beneficial impact on different Buchwald-Hartwig aminations, control reactions using water as the reaction solvent were conducted. Two different Buchwald-Hartwig aminations were performed in the presence and absence of HPMC in the reaction solvent (see Fig. S4). Reaction in water took prolonged time for completion. Nevertheless, the pictures of the reaction mixtures after $1 \mathrm{~h}$ reveal the formation of clumps if water was used as reaction solvent (see Fig. S5, A2 and B2). This could cause severe problems if the reaction is carried out on a larger scale. In contrast, stable suspensions were formed if HPMC/water was employed as reaction solvent (see Fig. S5, A1 and B1). Therefore, the use of HPMC as a solubilizer has two advantages: first, the homogeneity of the reaction mixture can be greatly improved which is important for scale up at the latest and second, no prior optimization is necessary for unknown substrates since the reaction is more robust for a broad range of substrates if HPMC is employed. Moreover, the analysis of the palladium catalyst revealed that nanoparticles are formed if HPMC is used which accelerates the reaction rate and prevents catalyst aggregation for example. 
A

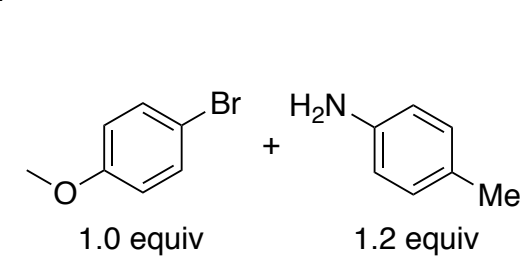

B<smiles>Brc1cccc2ccccc12</smiles>

1.0 equiv
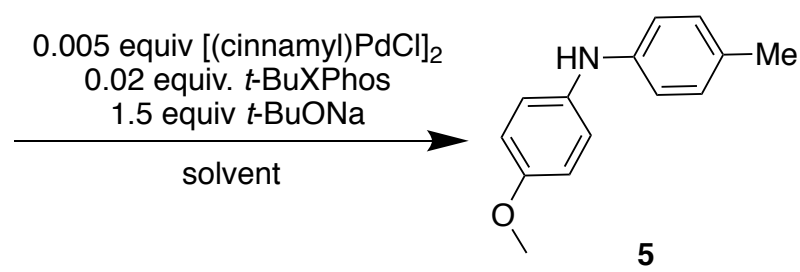

$86 \%$ (solvent $=2 \mathrm{wt} \%$ aq. $\mathrm{HPMC}, 15 \mathrm{~min})$ $80 \%$ (solvent $\left.=\mathrm{H}_{2} \mathrm{O}, 1 \mathrm{~h}\right)$
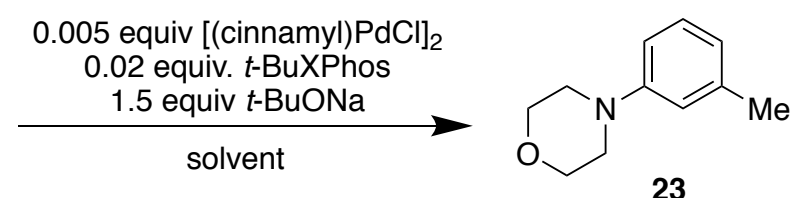

$88 \%$ (solvent $=2$ wt $\%$ aq. $\mathrm{HPMC}, 5 \mathrm{~min}$ ) $86 \%\left(\right.$ solvent $\left.=\mathrm{H}_{2} \mathrm{O}, 1 \mathrm{~h}\right)$

Figure S4. Comparison between $\mathrm{HPMC} / \mathrm{H}_{2} \mathrm{O}$ and pure $\mathrm{H}_{2} \mathrm{O}$ as reaction solvents for Buchwald-Hartwig aminations. General procedure (I) was followed using the catalyst, ligand and base stated above the reaction arrow.
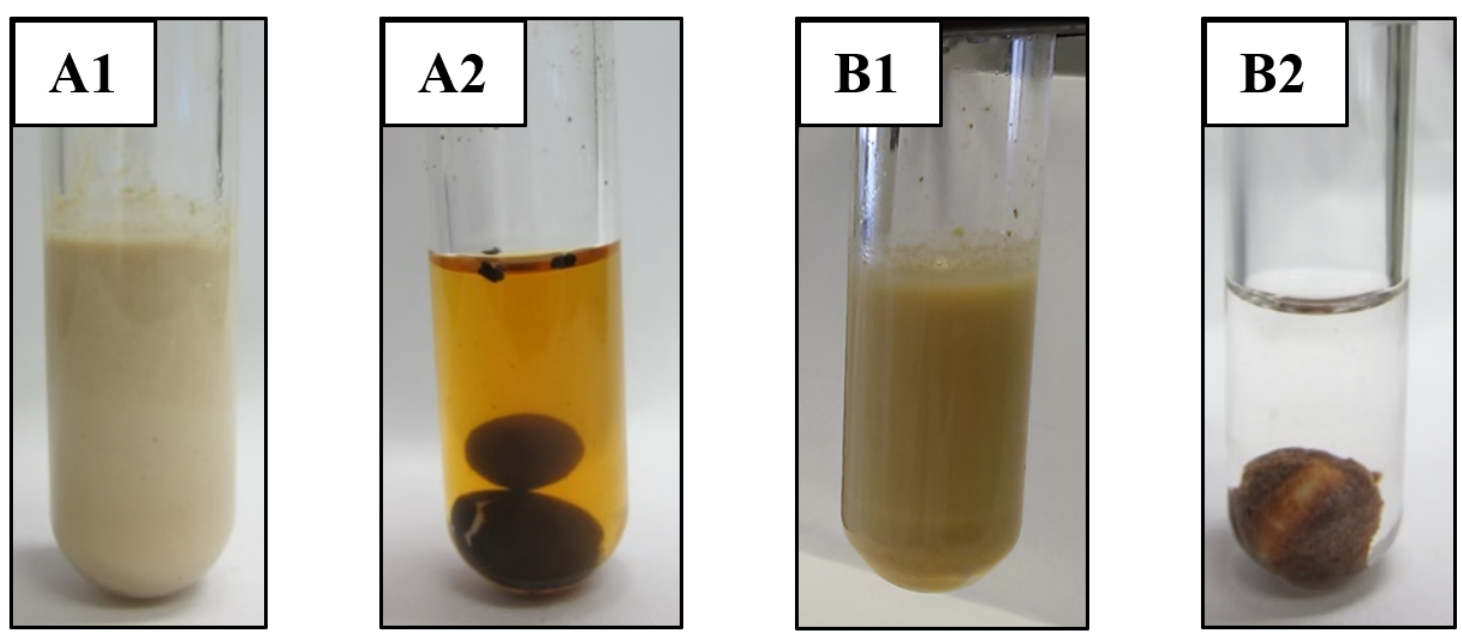

Figure S5. Comparison between $\mathrm{HPMC} / \mathrm{H}_{2} \mathrm{O}$ and pure $\mathrm{H}_{2} \mathrm{O}$ as reaction solvents for Buchwald-Hartwig aminations. Pictures of the reaction mixtures in water were taken after $1 \mathrm{~h}$. A1: reaction A (see Fig. S4) in $2 \mathrm{wt} \% \mathrm{HPMC} / \mathrm{H}_{2} \mathrm{O}$. A2: reaction A (see Fig. S4) in $\mathrm{H}_{2} \mathrm{O}$. B1: reaction B (see Fig. S4) in $2 \mathrm{wt} \% \mathrm{HPMC} / \mathrm{H}_{2} \mathrm{O}$. B2: reaction B (see Fig. S4) in $\mathrm{H}_{2} \mathrm{O}$. 


\section{Synthesis and Characterization of Amination Products}

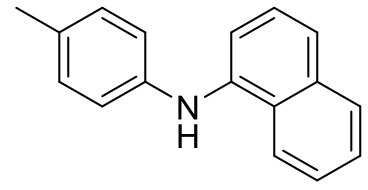

$N$-(p-tolyl)naphthalen-1-amine (4): The general procedure (I) was followed using sodium tert-butoxide $(0.75 \mathrm{mmol}, 72.1 \mathrm{mg}, 1.5$ equiv.), 2-di-tert-butylphosphino-2',4',6'-triisopropylbiphenyl ('BuXPhos) ligand $\quad(0.022 \mathrm{mmol}, \quad 9.3 \mathrm{mg}, \quad 4.4 \mathrm{~mol} \%), \quad$ bis[cinnamyl palladium(II)chloride] (0.005 mmol, $2.9 \mathrm{mg}, 1.1 \mathrm{~mol} \%)$, 1-bromonaphtalene (0.5 mmol, $104 \mathrm{mg}, 1.0$ equiv.) and $p$-toluidine ( $0.6 \mathrm{mmol}, 67.0 \mathrm{mg}, 1.25$ equiv.). The reaction was quenched (by addition of sat. aq. sodium sulfate solution) after 5 min and submitted to work-up and further purified via preparative HPLC (column: x-Bridge C8, 19x150 mm, eluents: water $\left.+0.1 \% \mathrm{NH}_{4} \mathrm{OH}(\mathrm{A}), \mathrm{MeCN}+0.1 \% \mathrm{NH}_{4} \mathrm{OH}(\mathrm{B})\right)$. The desired product was obtained in $94 \%$ isolated yield (115 mg) as a colorless oil. The spectral data are consistent with those reported in the literature (1).

${ }^{1}$ H NMR $\left(600 \mathrm{MHz}, \mathrm{CDCl}_{3}\right) \delta 8.02-8.00(\mathrm{~m}, 1 \mathrm{H}), 7.87-7.83(\mathrm{~m}, 1 \mathrm{H}), 7.53-7.44(\mathrm{~m}, 3 \mathrm{H})$, $7.36(\mathrm{t}, J=8.2 \mathrm{~Hz} 1 \mathrm{H}), 7.28(\mathrm{dd}, J=7.4,1.1 \mathrm{~Hz}, 2 \mathrm{H}), 7.11-7.06(\mathrm{~m}, 2 \mathrm{H}), 6.98-6.92$ (m, 2H), 5.89 (bs, 1H, NH), 2.31 (s, 3H) ppm.

${ }^{13}$ C NMR $\left(126 \mathrm{MHz}, \mathrm{CDCl}_{3}\right) \delta 141.8,139.7,134.7,130.5,129.9,128.65,127.1,126.2$, $126.1,125.6,122.1,121.6,118.6,114.2,20.7 \mathrm{ppm}$.<smiles>COc1ccc(Nc2ccc(C)cc2)cc1</smiles>

biphenyl ( $\left.{ }^{t} \mathrm{BuXPhos}\right)$ ligand (0.022 mmol, $\left.9.4 \mathrm{mg}, 4.4 \mathrm{~mol} \%\right)$, bis[cinnamyl palladium(II)chloride] (0.005 mmol, $2.9 \mathrm{mg}, 1.1 \mathrm{~mol} \%$ ), 4-bromoanisol (0.5 mmol, 94.0 $\mathrm{mg}, 1.0$ equiv.) and $p$-toluidine ( $0.6 \mathrm{mmol}, 67.3 \mathrm{mg}, 1.25$ equiv.) in $0.2 \mathrm{~mL} 2 \mathrm{wt} \% \mathrm{HPMC}$ solution. The reaction was quenched (by addition of sat. aq. sodium sulfate solution) after 15 min and submitted to work-up. The desired product was obtained in $97 \%$ isolated yield (104.1 mg) as pale-yellow solid. The spectral data are consistent with those reported in the literature (2). 
${ }^{1}$ H NMR (500 MHz, $\left.\mathrm{CDCl}_{3}\right)$ 8 7.08-6.96 (m, 4H), 6.89-6.77 (m, 4H), 5.37 (s, 1H), 3.77 (s, $3 \mathrm{H}), 2.27(\mathrm{~s}, 3 \mathrm{H}) \mathrm{ppm}$.

${ }^{13} \mathrm{C}$ NMR $\left(126 \mathrm{MHz}, \mathrm{CDCl}_{3}\right): \delta 154.8,142.4,136.7,129.8,129.3,121.1,116.6,114.7$, $55.6,20.6 \mathrm{ppm}$.<smiles>Cc1ccc(Nc2ccc(C#N)cc2)cc1</smiles>

4-(p-Tolylamino)benzonitrile (6): The general procedure (II) was followed using sodium tert-butoxide $(0.75 \mathrm{mmol}, 72.1 \mathrm{mg}$, 1.5 equiv.), 2-di-tert-butylphosphino-2',4',6'-triisopropyl biphenyl ('BuXPhos) ligand (0.022 mmol, $9.3 \mathrm{mg}, 4.4 \mathrm{~mol} \%)$, bis[cinnamyl palladium(II)chloride] ( $0.005 \mathrm{mmol}, 2.9 \mathrm{mg}, 1.1 \mathrm{~mol} \%)$, 4-bromobenzonitrile $(0.5 \mathrm{mmol}$, $91 \mathrm{mg}, \quad 1.0$ equiv. $)$ and $p$-toluidine $(0.6 \mathrm{mmol}, 67.0 \mathrm{mg}, 1.25$ equiv. $)$ in $0.2 \mathrm{~mL} 2 \mathrm{wt} \%$ HPMC solution. The reaction was quenched (by addition of sat. aq. sodium sulfate solution) after $5 \mathrm{~min}$ and submitted to work-up. The desired product was obtained in $86 \%$ isolated yield $(89.7 \mathrm{mg})$ as a yellow solid. The spectral data are consistent with those reported in the literature (3).

${ }^{1} \mathbf{H}$ NMR $\left(500 \mathrm{MHz}, \mathrm{CDCl}_{3}\right) \delta 7.52-7.39(\mathrm{~m}, 2 \mathrm{H}), 7.22-7.11(\mathrm{~m}, 2 \mathrm{H}), 7.12-7.02(\mathrm{~m}, 2 \mathrm{H})$, 6.94-6.81 (m, 2H), $5.98(\mathrm{~s}, 1 \mathrm{H}), 2.35$ (s, 3H) ppm.

${ }^{13}$ C NMR (126 MHz, $\left.\mathrm{CDCl}_{3}\right): 148.7,137.2,134.1,133.7,130.2,122.1,120.0,114.4$, 100.9, $20.9 \mathrm{ppm}$.<smiles>Cc1ccc(Nc2ccc(C(F)(F)F)cc2)cc1</smiles>

4-Methyl- $N$-(4-(trifluoromethyl)phenyl)aniline $\quad$ (7): The general procedure (II) was followed using sodium tert-butoxide (0.75 mmol, $72.4 \mathrm{mg}, 1.5$ equiv.), 2-di-tert-butylphosphino2',4',6'-triisopropylbiphenyl ('BuXPhos) ligand (0.022 mmol, $9.4 \mathrm{mg}, 4.4 \mathrm{~mol} \%$ ), bis[cinnamyl palladium(II)chloride] $\quad(0.005$ mmol, $\quad 2.9 \quad \mathrm{mg}, \quad 1.1 \quad \mathrm{~mol} \%), \quad 4-$ bromobenzotrifluoride ( $0.5 \mathrm{mmol}, 113 \mathrm{mg}, 1.0$ equiv.) and $p$-toluidine $(0.6 \mathrm{mmol}, 67.3 \mathrm{mg}$, 1.25 equiv.) in $0.2 \mathrm{~mL} 2 \mathrm{wt} \%$ HPMC solution. The reaction was quenched (by addition of sat. aq. sodium sulfate solution) after 5 min and submitted to work-up. The desired product was obtained in $95 \%$ isolated yield $(120 \mathrm{mg})$ as a white solid. The spectral data are consistent with those reported in the literature (3). 
${ }^{1} \mathbf{H}$ NMR $\left(500 \mathrm{MHz}, \mathrm{CDCl}_{3}\right) \delta 7.44(\mathrm{~d}, J=8.5 \mathrm{~Hz}, 2 \mathrm{H}), 7.15(\mathrm{~d}, J=8.1 \mathrm{~Hz}, 2 \mathrm{H}), 7.10$ $7.01(\mathrm{~m}, 2 \mathrm{H}), 6.97$ (d, $J=8.5 \mathrm{~Hz}, 2 \mathrm{H}), 5.82(\mathrm{bs}, 1 \mathrm{H}, \mathrm{NH}), 2.34$ (s, 3H) ppm.

${ }^{13}$ C NMR (126 MHz, $\left.\mathrm{CDCl}_{3}\right): 147.5,138.3,133.0,130.1,126.7$ (q, $\left.J=3.8 \mathrm{~Hz}\right), 121.1$, 120.9, 114.6, $20.81 \mathrm{ppm}$.

${ }^{19}$ F NMR $\left(471 \mathrm{MHz}, \mathrm{CDCl}_{3}\right) \delta-61.37(\mathrm{~s}, 3 \mathrm{~F}) \mathrm{ppm}$.<smiles>O=[N+]([O-])c1ccc(Nc2ccc(I)cc2)cc1</smiles>

4-Methyl- $N$-(4-nitrophenyl)aniline (8): The general procedure (I) was followed using sodium tertbutoxide (0.75 mmol, $72.1 \mathrm{mg}, \quad 1.5$ equiv.), 2-di-tertbutylphosphino-2',4',6'-triisopropylbiphenyl ('BuXPhos) ligand (0.022 mmol, $9.3 \mathrm{mg}, 4.4$ mol\%), bis[cinnamyl palladium(II)chloride] (0.005 mmol, $2.9 \mathrm{mg}, 1.1 \mathrm{~mol} \%$ ), 1-bromo-4nitrobenzene ( $0.5 \mathrm{mmol}, 101 \mathrm{mg}, 1.0$ equiv.) and $p$-toluidine $(0.6 \mathrm{mmol}, 67.0 \mathrm{mg}, 1.25$ equiv.). The reaction was quenched (by addition of sat. aq. sodium sulfate solution) after 3 min and submitted to work-up. The desired product was obtained in $83 \%$ isolated yield $(94.5 \mathrm{mg})$ as an orange solid. The spectral data are consistent with those reported in the literature (4).

${ }^{1} \mathbf{H}$ NMR $\left(500 \mathrm{MHz}, \mathrm{CDCl}_{3}\right) \delta 8.13-8.05(\mathrm{~m}, 2 \mathrm{H}), 7.22-7.17(\mathrm{~m}, 2 \mathrm{H}), 7.13-7.08(\mathrm{~m}, 2 \mathrm{H})$, 6.89-6.83 (m, 2H), 6.24 (bs, 1H, NH), 2.36 (s, 3H) ppm.

${ }^{13}$ C NMR (126 MHz, $\mathrm{CDCl}_{3}$ ): 150.8, 139.4, 136.7, 134.8, 130.3, 126.3, 122.7, 113.2, 20.9 ppm.

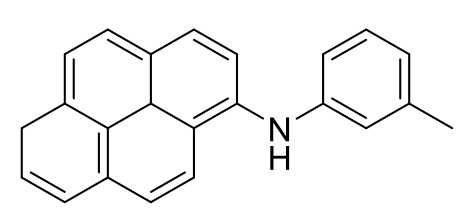

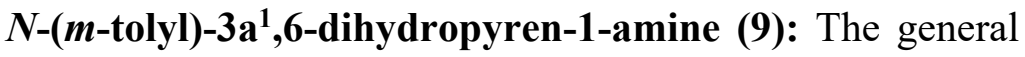
procedure (I) was followed using sodium tert-butoxide ( 0.75 mmol, $72.5 \mathrm{mg}, 1.5$ equiv.), 2-di-tert-butylphosphino2',4',6'-triisopropylbiphenyl ('BuXPhos) ligand (0.022 mmol, $9.4 \mathrm{mg}, 4.4 \mathrm{~mol} \%$ ), bis[cinnamyl palladium(II)chloride] (0.005 mmol, $2.9 \mathrm{mg}, 1.1$ mol\%), 3-bromotoluene ( $0.5 \mathrm{mmol}, 86 \mathrm{mg}, 61 \mu \mathrm{L}, 1.0$ equiv.) and 1-aminopyrene (0.6 mmol, $137 \mathrm{mg}, 1.25$ equiv.). The reaction was quenched (by addition of sat. aq. sodium sulfate solution) after $15 \mathrm{~min}$ and submitted to work-up. The desired product was obtained in $93 \%$ isolated yield $(160 \mathrm{mg})$ as a yellow solid. 
${ }^{1} \mathbf{H}$ NMR $\left(500 \mathrm{MHz}, \mathrm{CDCl}_{3}\right) \delta 8.22-8.03(\mathrm{~m}, 4 \mathrm{H}), 8.01-7.89(\mathrm{~m}, 5 \mathrm{H}), 7.16(\mathrm{t}, J=7.4 \mathrm{~Hz}$, 1H), 6.88-6.83 (m, 2H), 6.76 (d, J=7.8 Hz, 1H), 6.18 (bs, 1H, NH), 2.29 (s, 3H) ppm.

${ }^{13}$ C NMR $\left(126 \mathrm{MHz}, \mathrm{CDCl}_{3}\right) \delta 144.9,139.4,136.8,131.8,131.4,129.3,127.4,127.2$, 127.0, 126.1, 126.0, 125.6, 125.6, 125.2, 124.8, 124.4, 123.2, 121.6, 121.3, 119.1, 117.7, $114.2,21.6 \mathrm{ppm}$.

HRMS (ESI) $\mathrm{m} / \mathrm{z}$ calcd. for $\mathrm{C}_{23} \mathrm{H}_{18} \mathrm{~N}\left([\mathrm{M}+\mathrm{H}]^{+}\right) 308.1434$, found 308.1420 .

IR (film) vmax 3417, 2360, 1597, 1515, 1481, 1313, 1267, 1163, 842, 826, 763, 710, 689, $678 \mathrm{~cm}^{-1}$.

Melting point $128.1^{\circ} \mathrm{C}$

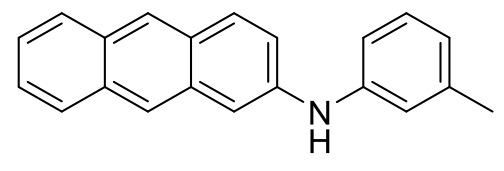

$N$-(m-tolyl)anthracen-2-amine (10): The general procedure (II) was followed using sodium tertbutoxide (0.75 mmol, $72.5 \mathrm{mg}, 1.5$ equiv.), 2-di-tertbutylphosphino-2',4',6'-triisopropylbiphenyl ('BuXPhos) ligand (0.022 mmol, $9.4 \mathrm{mg}, 4.4$ mol\%), bis[cinnamyl palladium(II)chloride] (0.005 mmol, $2.9 \mathrm{mg}, 1.1 \mathrm{~mol} \%), 3-$ bromotoluene ( $0.5 \mathrm{mmol}, 86 \mathrm{mg}, 61 \mu \mathrm{L}, 1.0$ equiv.) and 2-aminoanthracene $(0.6 \mathrm{mmol}$, $121 \mathrm{mg}, 1.25$ equiv.). The reaction was quenched (by addition of sat. aq. sodium sulfate solution) after $15 \mathrm{~min}$ and submitted to work-up. The desired product was obtained in $94 \%$ isolated yield (149.2 $\mathrm{mg}$ ) as an orange-brown solid.

${ }^{1} \mathbf{H}$ NMR $\left(500 \mathrm{MHz}, \mathrm{CDCl}_{3}\right) \delta 8.31(\mathrm{~s}, 1 \mathrm{H}), 8.17(\mathrm{~s}, 1 \mathrm{H}), 7.98-7.87(\mathrm{~m}, 3 \mathrm{H}), 7.55-7.52(\mathrm{~m}$, 1H), $7.41(\mathrm{~m}, 2 \mathrm{H}), 7.25-7.20(\mathrm{~m}, 2 \mathrm{H}), 7.07-7.02(\mathrm{~m}, 2 \mathrm{H}), 6.87-6.83(\mathrm{~m}, 1 \mathrm{H}), 5.89$ (bs, $1 \mathrm{H}, \mathrm{NH}), 2.37$ (s, 3H) ppm.

${ }^{13}$ C NMR $\left(126 \mathrm{MHz}, \mathrm{CDCl}_{3}\right) \delta 142.5,140.3,139.4,132.9,132.4,130.3,129.6,129.3$, $128.4,128.3,127.6,126.2,125.5,124.3,123.6,122.7,121.4,119.5,115.9,108.9$, $21.6 \mathrm{ppm}$.

HRMS (ESI) $\mathrm{m} / \mathrm{z}$ calcd. for $\mathrm{C}_{21} \mathrm{H}_{18} \mathrm{~N}\left([\mathrm{M}+\mathrm{H}]^{+}\right)$284.1434, found 284.1431 .

IR (film) vmax 3408, 1630, 1582, 1482, 1308, 1166, 956, 890, 767, 737, 697, $639 \mathrm{~cm}^{-1}$.

Melting point $169.5^{\circ} \mathrm{C}$ 


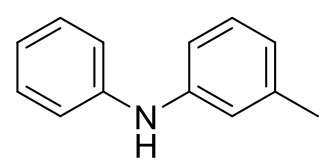

3-Methyl- $N$-phenylaniline (11): The general procedure (II) was followed using sodium tert-butoxide $(0.75 \mathrm{mmol}, 72.5 \mathrm{mg}$, 1.5 equiv.), 2-di-tert-butylphosphino-2',4',6'-triisopropylbiphenyl ('BuXPhos) ligand $\quad(0.022 \mathrm{mmol}, \quad 9.4 \mathrm{mg}, \quad 4.4 \quad \mathrm{~mol} \%)$, bis[cinnamyl palladium(II)chloride] (0.005 mmol, $2.9 \mathrm{mg}, 1.1 \mathrm{~mol} \%)$, 3-bromotoluene $(0.5 \mathrm{mmol}$, $86 \mathrm{mg}, 61 \mu \mathrm{L}, 1.0$ equiv.) and aniline (0.6 mmol, $59 \mathrm{mg}, 57 \mu \mathrm{L}, 1.25$ equiv.) in $0.4 \mathrm{~mL} 2 \mathrm{wt} \%$ HPMC solution. The reaction was quenched (by addition of sat. aq. sodium sulfate solution) after $5 \mathrm{~min}$ and submitted to work-up. The desired product was obtained in $89 \%$ isolated yield $(81.6 \mathrm{mg}$ ) as colorless crystals. The spectral data are consistent with those reported in the literature (5).

${ }^{1} \mathbf{H}$ NMR $\left(500 \mathrm{MHz}, \mathrm{CDCl}_{3}\right) \delta$ 7.29-7.20 (m, 2H), 7.18-7.11 (m, 1H), 7.09-7.02 (m, 2H), 6.94-6.84 (m, 3H), $6.74(\mathrm{~d}, J=9.0 \mathrm{~Hz}, 1 \mathrm{H}), 5.61$ (s, 1H, NH), 2.30 (s, 3H) ppm.

${ }^{13} \mathbf{C}$ NMR $\left(126 \mathrm{MHz}, \mathrm{CDCl}_{3}\right) \delta 143.3,143.1,139.3,129.4,129.2,121.9,120.9,118.6$, $117.9,114.9,21.5 \mathrm{ppm}$.

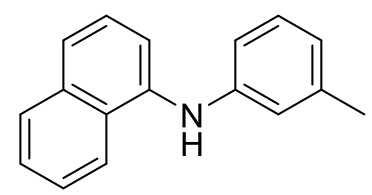

$N$-(m-tolyl)naphthalen-1-amine (12): The general procedure (I) was followed using sodium tert-butoxide $(0.75 \mathrm{mmol}, 72.5 \mathrm{mg}, 1.5$ equiv.), 2-di-tert-butylphosphino-2',4',6'-triisopropylbiphenyl ('BuXPhos) ligand (0.022 mmol, $9.4 \mathrm{mg}, 4.4 \mathrm{~mol} \%$ ), bis[cinnamyl palladium(II)chloride] (0.005 mmol, $2.9 \mathrm{mg}, 1.1 \mathrm{~mol} \%$ ), 3-bromotoluene ( $0.5 \mathrm{mmol}, 86 \mathrm{mg}, 61 \mu \mathrm{L}, 1.0$ equiv.) and 1-aminonaphthalene ( $0.6 \mathrm{mmol}, 90 \mathrm{mg}, 1.25$ equiv.). The reaction was quenched (by addition of sat. aq. sodium sulfate solution) after $15 \mathrm{~min}$ and submitted to work-up. The desired product was obtained in $96 \%$ isolated yield $(113 \mathrm{mg})$ as colorless oil. The spectral data are consistent with those reported in the literature (6).

${ }^{1} \mathbf{H}$ NMR $\left(600 \mathrm{MHz}, \mathrm{CDCl}_{3}-d\right) \delta 8.01(\mathrm{~d}, J=8.0 \mathrm{~Hz}, 1 \mathrm{H}), 7.86(\mathrm{~d}, J=7.0 \mathrm{~Hz}, 1 \mathrm{H}), 7.56(\mathrm{~d}$, $J=8.0 \mathrm{~Hz}, 1 \mathrm{H}), 7.52-7.44(\mathrm{~m}, 2 \mathrm{H}), 7.42-7.35(\mathrm{~m}, 2 \mathrm{H}), 7.14(\mathrm{td}, J=8.0,1.4 \mathrm{~Hz}, 1 \mathrm{H})$, 6.85-6.78 (m, 2H), 6.74 (d, $J=7.0 \mathrm{~Hz}, 1 \mathrm{H}), 5.89$ (bs, 1H, NH), 2.30 (s, 3H) ppm.

${ }^{13}$ C NMR (126 MHz, $\left.\mathrm{CDCl}_{3}\right) \delta 144.6,139.2,138.8,134.7,129.2,128.5,127.7,126.1$, $126.0,125.7,122.9,121.8,121.5,118.2,115.9,114.6,21.5 \mathrm{ppm}$. 


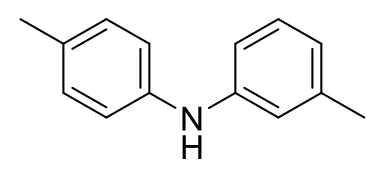

3-Methyl- $N$-(p-tolyl)aniline (13): The general procedure (I) was followed using sodium tert-butoxide $(0.75 \mathrm{mmol}, 72.5 \mathrm{mg}$, 1.5 equiv.), 2-di-tert-butylphosphino-2',4',6'-triisopropylbiphenyl ('BuXPhos) ligand $(0.022 \mathrm{mmol}, \quad 9.4 \mathrm{mg}, \quad 4.4 \quad \mathrm{~mol} \%)$, bis[cinnamyl palladium(II)chloride] (0.005 mmol, $2.9 \mathrm{mg}, 1.1 \mathrm{~mol} \%)$, 3-bromotoluene $(0.5 \mathrm{mmol}$, $86 \mathrm{mg}, 61 \mu \mathrm{L}, 1.0$ equiv.) and $p$-toluidine ( $0.6 \mathrm{mmol}, 67 \mathrm{mg}, 1.25$ equiv.). The reaction was quenched (by addition of sat. aq. sodium sulfate solution) after 5 min and submitted to work-up. The desired product was obtained in $91 \%$ isolated yield $(90 \mathrm{mg}$ ) as colorless oil. The spectral data are consistent with those reported in the literature (7).

${ }^{1} \mathbf{H}$ NMR $\left(500 \mathrm{MHz}, \mathrm{CDCl}_{3}\right) \delta$ 7.16-7.05 (m, 3H), 7.03-6.95 (m, 2H), $6.82(\mathrm{~m}, 2 \mathrm{H})$, $6.70(\mathrm{~d}, J=7.4 \mathrm{~Hz}, 1 \mathrm{H}), 5.54$ (bs, 1H, NH), 2.30 (s, 3H), 2.29 (s, 3H) ppm.

${ }^{13} \mathrm{C}$ NMR $\left(126 \mathrm{MHz}, \mathrm{CDCl}_{3}\right) \delta 143.9,140.4,139.2,130.8,129.8,129.1,121.2,118.9$, $117.5,114.0,21.5,20.7 \mathrm{ppm}$.

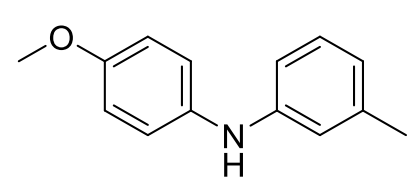

$N$-(4-methoxyphenyl)-3-methylaniline (14): The general procedure (I) was followed using sodium tert-butoxide (0.75 mmol, $72.5 \mathrm{mg}, 1.5$ equiv.), 2-di-tert-butylphosphino2',4',6'-triisopropylbiphenyl ('BuXPhos) ligand (0.022 mmol, $9.4 \mathrm{mg}, 4.4 \mathrm{~mol} \%$ ), bis[cinnamyl palladium(II)chloride] $\quad(0.005$ mmol, $2.9 \quad \mathrm{mg}, \quad 1.1 \mathrm{~mol} \%), \quad 3-$ bromotoluene ( $0.5 \mathrm{mmol}, 86 \mathrm{mg}, 61 \mu \mathrm{L}, 1.0$ equiv.) and $p$-anisidine $(0.6 \mathrm{mmol}, 77 \mathrm{mg}$, 1.25 equiv.). The reaction was quenched (by addition of sat. aq. sodium sulfate solution) after 5 min and submitted to work-up. The desired product was obtained in $93 \%$ isolated yield $(100 \mathrm{mg})$ as white solid. The spectral data are consistent with those reported in the literature (7).

${ }^{1} \mathbf{H}$ NMR $\left(500 \mathrm{MHz}, \mathrm{CDCl}_{3}\right) \delta$ 7.19-7.04 (m, 3H), 6.94-6.85 (m, 2H), 6.77-6.73 (m, 2H), 6.69 (d, $J=7.2 \mathrm{~Hz}, 1 \mathrm{H}), 5.46$ (bs, 1H, NH), 3.83 (s, 3H), 2.32 (s, 3H) ppm.

${ }^{13} \mathrm{C}$ NMR $\left(126 \mathrm{MHz}, \mathrm{CDCl}_{3}\right) \delta 155.2,145.2,139.2,135.9,129.2,122.2,120.5,116.4$, $114.7,112.8,55.6,21.6 \mathrm{ppm}$. 
$\mathrm{F}_{3} \mathrm{C}$<smiles>Cc1ccc(Nc2cccc(C)c2)cc1</smiles>
3-Methyl- $N$-(4-(trifluoromethyl)phenyl)aniline (15): The general procedure (II) was followed using sodium tert-butoxide (0.75 mmol, $72.5 \mathrm{mg}, 1.5$ equiv.), 2-di-tert-butylphosphino2',4',6'-triisopropylbiphenyl ('BuXPhos) ligand (0.022 mmol, $9.4 \mathrm{mg}, 4.4 \mathrm{~mol} \%$ ), bis[cinnamyl palladium(II)chloride] (0.005 mmol, $2.9 \mathrm{mg}, 1.1 \mathrm{~mol} \%)$, 3-bromotoluene (0.5 mmol, $86 \mathrm{mg}, 61 \mu \mathrm{L}, 1.0$ equiv.) and 4-aminobenzotrifluoride (0.6 mmol, $101 \mathrm{mg}, 1.25$ equiv.) in $0.2 \mathrm{~mL} 2 \mathrm{wt} \%$ HPMC solution. The reaction was quenched (by addition of sat. aq. sodium sulfate solution) after $5 \mathrm{~min}$ and submitted to work-up. The desired product was obtained in $92 \%$ isolated yield $(116 \mathrm{mg})$ as colorless oil. The spectral data are consistent with those reported in the literature (8).

${ }^{1}$ H NMR $\left(500 \mathrm{MHz}, \mathrm{CDCl}_{3}\right) \delta 7.45(\mathrm{~d}, J=8.5 \mathrm{~Hz}, 2 \mathrm{H}), 7.23-7.18(\mathrm{~m}, 1 \mathrm{H}), 7.01(\mathrm{~d}, J=8.5$ $\mathrm{Hz}, 2 \mathrm{H}), 6.97-6.92(\mathrm{~m}, 2 \mathrm{H}), 6.88-6.85$ (m, 1H), 5.84 (bs, 1H, NH), 2.33 (s, 3H) ppm.

${ }^{13}$ C NMR $\left(126 \mathrm{MHz}, \mathrm{CDCl}_{3}\right) \delta 146.9,141.1,139.5,129.3,126.7$ (q, $\left.J=3.8 \mathrm{~Hz}\right), 123.8$, $120.8,117.1,115.3,21.5 \mathrm{ppm}$.

${ }^{19}$ F NMR $\left(471 \mathrm{MHz}, \mathrm{CDCl}_{3}\right) \delta-61.35(\mathrm{~s}, 3 \mathrm{~F}) \mathrm{ppm}$.<smiles>Cc1cccc(Nc2ccc(C#N)cc2)c1</smiles>

4-(m-Tolylamino)benzonitrile (16): The general procedure (I) was followed using sodium tert-butoxide $(0.75 \mathrm{mmol}, 72.5 \mathrm{mg}$, 1.5 equiv.), 2-di-tert-butylphosphino-2', 4',6'triisopropylbiphenyl ( $\left.{ }^{t} \mathrm{BuXPhos}\right)$ ligand (0.022 mmol, $9.4 \mathrm{mg}, 4.4 \mathrm{~mol} \%$ ), bis[cinnamyl palladium(II)chloride] (0.005 mmol, $2.9 \mathrm{mg}, 1.1 \mathrm{~mol} \%$ ), 3-bromotoluene (0.5 mmol, $86 \mathrm{mg}, 61 \mu \mathrm{L}, 1.0$ equiv.) and 4-aminobenzonitrile ( $0.6 \mathrm{mmol}, 74.3 \mathrm{mg}, 1.25$ equiv.). The reaction was quenched (by addition of sat. aq. sodium sulfate solution) after $15 \mathrm{~min}$ and submitted to work-up. The desired product was obtained in $88 \%$ isolated yield $(92.4 \mathrm{mg})$ as a white solid.

${ }^{1}$ H NMR (600 MHz, CDCl $) \delta$ 7.53-7.47 (m, 2H), 7.28-7.25 (m, 1H), 7.02-6.94 (m, 5H), $5.98(\mathrm{bs}, 1 \mathrm{H}), 2.38(\mathrm{~s}, 3 \mathrm{H}) \mathrm{ppm}$.

${ }^{13}$ C NMR (126 MHz, $\left.\mathrm{CDCl}_{3}\right) \delta$ 148.2, 139.9, 139.6, 133.7, 129.4, 124.8, 121.9, 119.9, $118.3,114.9,101.3,21.5 \mathrm{ppm}$. 
IR (film) vmax 3327, 2216, 1605, 1590, 1531, 1491, 1337, 1165, 827, 771, $692 \mathrm{~cm}^{-1}$.

HRMS (ESI) m/z calcd. for $\mathrm{C}_{14} \mathrm{H}_{13} \mathrm{~N}_{2}\left([\mathrm{M}+\mathrm{H}]^{+}\right)$209.1078, found 209.1073 .

Melting point $79.5^{\circ} \mathrm{C}$

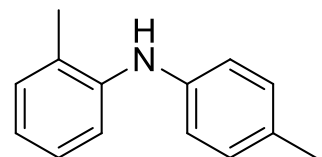

2-Methyl- $N$-(p-tolyl)aniline (17): The general procedure (II) was followed using $p$-toludine $(67.2 \mathrm{mg}, 0.652 \mathrm{mmol}, 1.25$ equiv), $[(\text { cinnamyl }) \mathrm{PdCl}]_{2}(2.9 \quad \mathrm{mg}, \quad 5.5 \mu \mathrm{mol}, \quad 0.011 \quad$ equiv. $)$, ${ }^{t} \mathrm{BuXPhos}$ (9.3 mg, $0.022 \mathrm{mmol}, 0.044$ equiv.), $\mathrm{NaO}{ }^{t} \mathrm{Bu}$ (72 mg, $0.75 \mathrm{mmol}, 1.5$ equiv.) and 2-bromotoluene ( $61 \mu \mathrm{L}, 0.5 \mathrm{mmol}, 1.0$ equiv.) in $0.2 \mathrm{~mL} 2 \mathrm{wt} \% \mathrm{HPMC}$ solution. The reaction was quenched (by addition of sat. aq. sodium sulfate solution) after $20 \mathrm{~min}$ and submitted to work-up. The column was eluted with $0-15 \%$ EtOAc in heptane $(30 \mathrm{~mL} / \mathrm{min})$. The product was obtained in $81 \%$ yield $(80 \mathrm{mg})$ as a clear oil. The spectral data are consistent with those reported in the literature (9).

${ }^{1} \mathbf{H}$ NMR $\left(500 \mathrm{MHz}, \mathrm{CDCl}_{3}\right) \delta$ 7.20-7.14 (m, 2H), 7.13-7.05 (m, 3H), 6.94-6.85 (m, 3H), $5.30(\mathrm{br} \mathrm{s}, 1 \mathrm{H}), 2.30(\mathrm{~s}, 3 \mathrm{H}), 2.25(\mathrm{~s}, 3 \mathrm{H}) \mathrm{ppm}$.

${ }^{13} \mathrm{C}$ NMR $\left(126 \mathrm{MHz}, \mathrm{CDCl}_{3}\right) \delta 142.1,141.2,130.9,130.5,129.9,127.1,126.8,121.1$, 118.7, 117.3, 20.7, $17.9 \mathrm{ppm}$.

2-Methoxy- $\mathrm{N}$-( $\boldsymbol{p}$-tolyl)aniline (18): The general procedure (II) was
followed using $p$-toludine $(67.2 \mathrm{mg}, 0.652 \mathrm{mmol}, 1.25$ equiv),
$\left[(\text { cinnamyl)PdCl}]_{2}(2.9 \quad \mathrm{mg}, \quad 5.5 \mu \mathrm{mol}, \quad 0.011 \quad\right.$ equiv.), ${ }^{t} \mathrm{BuXPhos}$ (9.3 mg, $0.022 \mathrm{mmol}, 0.044$ equiv.), $\mathrm{NaO}{ }^{t} \mathrm{Bu}$ (72 mg, $0.75 \mathrm{mmol}, 1.5$ equiv.) and 1-bromo-2-methoxybenzene ( $63 \mu \mathrm{L}, 0.5 \mathrm{mmol}, 1.0$ equiv.) in $0.2 \mathrm{~mL} 2 \mathrm{wt} \% \mathrm{HPMC}$ solution. The reaction was quenched (by addition of sat. aq. sodium sulfate solution) after 20 min and submitted to work-up. The column was eluted with 0-30\% EtOAc in heptane $(18 \mathrm{~mL} / \mathrm{min})$. The product was obtained in $97 \%$ yield $(108 \mathrm{mg})$ as a pale-pink oil. The spectral data are consistent with those reported in the literature (10).

${ }^{1} \mathbf{H}$ NMR $\left(500 \mathrm{MHz}, \mathrm{CDCl}_{3}\right) \delta 7.21(\mathrm{dd}, J=7.7 \mathrm{~Hz}, 1.7 \mathrm{~Hz}, 1 \mathrm{H}), 7.13-7.04(\mathrm{~m}, 4 \mathrm{H}), 6.90-$ $6.78(\mathrm{~m}, 3 \mathrm{H}), 6.06$ (br s, 1H), 3.89 (s, 3H), 2.31 (s, 3H) ppm. 
${ }^{13}$ C NMR (126 MHz, $\left.\mathrm{CDCl}_{3}\right) \delta 148.0,140.1,134.0,131.1,129.9,121.0,119.7,119.3$, $113.8,110.5,77.4,76.9,55.7,20.8 \mathrm{ppm}$.<smiles>COc1cc(C)c(Nc2ccc(C)cc2)c(C)c1</smiles>

4-Methoxy-2,6-dimethyl- $N$-(p-tolyl)aniline (19): The general procedure (II) was followed using $p$-toludine $(67.2 \mathrm{mg}$, $0.652 \mathrm{mmol}, \quad 1.25$ equiv $), \quad[(\text { cinnamyl }) \mathrm{PdCl}]_{2}(13.0 \mathrm{mg}$, $0.025 \mathrm{mmol}, 0.05$ equiv.), ${ }^{t} \mathrm{BuXPhos}$ (21.3 mg, $0.05 \mathrm{mmol}, 0.1$ equiv.), $\mathrm{NaO}{ }^{t} \mathrm{Bu}$ (72 mg, $0.75 \mathrm{mmol}, 1.5$ equiv.) and 2-bromo-5-methoxy-1,3-dimethylbenzene ( $83 \mu \mathrm{L}, 0.5 \mathrm{mmol}$, 1.0 equiv.) in $0.2 \mathrm{~mL} 2 \mathrm{wt} \%$ HPMC solution. The reaction was quenched (by addition of sat. aq. sodium sulfate solution) after $3 \mathrm{~h}$ and submitted to work-up. The column was eluted with $0-30 \%$ EtOAc in heptane $(18 \mathrm{~mL} / \mathrm{min})$. The product was obtained in $82 \%$ yield (99 $\mathrm{mg})$ as a white solid.

${ }^{1} \mathbf{H}$ NMR $\left(500 \mathrm{MHz}, \mathrm{CDCl}_{3}\right) \delta$ 7.01-6.91 (m, 2H), $6.66(\mathrm{~s}, 2 \mathrm{H}), 6.42-6.35(\mathrm{~m}, 2 \mathrm{H})$, 4.93 (br s, 1H), $3.80(\mathrm{~s}, 3 \mathrm{H}), 2.23(\mathrm{~s}, 3 \mathrm{H}), 2.18(\mathrm{~s}, 6 \mathrm{H}) \mathrm{ppm}$.

${ }^{13} \mathrm{C}$ NMR $\left(126 \mathrm{MHz}, \mathrm{CDCl}_{3}\right) \delta 157.3,144.9,137.9,131.7,129.9,127.0,113.6,113.1$, $55.4,20.5,18.7$ ppm.

HRMS (ESI) m/z calcd. for $\mathrm{C}_{16} \mathrm{H}_{20} \mathrm{NO}\left([\mathrm{M}+\mathrm{H}]^{+}\right)$242.1539, found 242.1543.

IR (film) vmax 3382, 1607, 1512, 1297, 1253, 1154, 1066, 992, 887, 846, $804 \mathrm{~cm}^{-1}$.

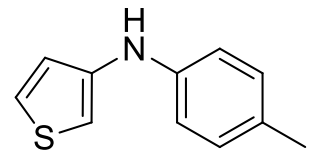

$N$-(p-tolyl)thiophen-3-amine (20): The general procedure (II) was followed using $p$-toludine $(67.2 \mathrm{mg}, \quad 0.652 \mathrm{mmol}, \quad 1.25$ equiv $)$, $[(\text { cinnamyl }) \mathrm{PdCl}]_{2}(2.90 \mathrm{mg}, \quad 5.5 \mu \mathrm{mol}$, 0.011 equiv.), ${ }^{t} \mathrm{BuXPhos}$ (9.3 mg, $0.022 \mathrm{mmol}, 0.044$ equiv.), $\mathrm{NaO}{ }^{t} \mathrm{Bu}$ (72 mg, $0.75 \mathrm{mmol}, 1.5$ equiv.) and 3-bromothiophene ( $47 \mu \mathrm{L}, 0.5 \mathrm{mmol}, 1.0$ equiv.) in $0.2 \mathrm{~mL} 2 \mathrm{wt} \% \mathrm{HPMC}$ solution. The reaction was quenched (by addition of sat. aq. sodium sulfate solution) after 20 min and submitted to work-up. The column was eluted with 0-30\% EtOAc in heptane $(18 \mathrm{~mL} / \mathrm{min})$. The product was obtained in $97 \%$ yield $(92 \mathrm{mg})$ as a white solid. The spectral data are consistent with those reported in the literature (11).

${ }^{1}$ H NMR $\left(500 \mathrm{MHz}, \mathrm{CDCl}_{3}\right) \delta 7.24(\mathrm{dd}, J=5.1 \mathrm{~Hz}, 3.1 \mathrm{~Hz}, 1 \mathrm{H}), 7.09-7.03$ (m, 2H), 6.93$6.85(\mathrm{~m}, 3 \mathrm{H}), 6.66$ (dd, $J=3.1 \mathrm{~Hz}, 1.5 \mathrm{~Hz}, 1 \mathrm{H}), 5.63$ (br s, 1H), 2.28 (s, 3H) ppm. 
${ }^{13} \mathbf{C}$ NMR $\left(126 \mathrm{MHz}, \mathrm{CDCl}_{3}\right) \delta 142.5,142.2,130.0,129.7,125.2,122.6,116.4,105.1,20.7$ ppm.<smiles>Cc1cccc(Nc2ncccn2)c1</smiles>
$N$-(m-tolyl)pyrimidin-2-amine (21): The general procedure (II) was followed using sodium tert-butoxide $(0.75 \mathrm{mmol}, 72.5 \mathrm{mg}, 1.5$ equiv.), 2-di-tert-butylphosphino-2', 4',6'-triisopropylbiphenyl $\left({ }^{t} \mathrm{BuXPhos}\right) \quad$ ligand $(0.022 \mathrm{mmol}, \quad 9.4 \mathrm{mg}, \quad 4.4 \quad \mathrm{~mol} \%)$, bis[cinnamyl palladium(II)chloride] (0.005 mmol, $2.9 \mathrm{mg}, 1.1 \mathrm{~mol} \%)$, 3-bromotoluene (0.5 mmol, $86 \mathrm{mg}, 61 \mu \mathrm{L}, 1.0$ equiv.) and 2-aminopyrimidine ( $0.5 \mathrm{mmol}, 48 \mathrm{mg}, 1.00$ equiv.) in $0.2 \mathrm{~mL}$ $2 \mathrm{wt} \%$ HPMC solution. The reaction was quenched (by addition of sat. aq. sodium sulfate solution) after $15 \mathrm{~h}$ and submitted to work-up. The desired product was obtained in $76 \%$ isolated yield $(71 \mathrm{mg})$ as a yellow oil. The spectral data are consistent with those reported in the literature (8).

${ }^{1} \mathbf{H}$ NMR $\left(600 \mathrm{MHz}, \mathrm{CDCl}_{3}\right) \delta 8.41(\mathrm{~d}, J=5.0 \mathrm{~Hz}, 2 \mathrm{H}), 7.44-7.41(\mathrm{~m}, 2 \mathrm{H}), 7.23(\mathrm{t}, J=7.9$ $\mathrm{Hz}, 1 \mathrm{H}), 6.89-6.87(\mathrm{~m}, 1 \mathrm{H}), 6.71(\mathrm{t}, J=5.0 \mathrm{~Hz}, 1 \mathrm{H}), 2.37(\mathrm{~s}, 3 \mathrm{H}) \mathrm{ppm}$.

${ }^{13} \mathrm{C}$ NMR $\left(126 \mathrm{MHz}, \mathrm{CDCl}_{3}\right) \delta 160.3,158.0,139.3,138.8,128.8,123.7,120.3,116.8$, 112.4, $21.6 \mathrm{ppm}$.

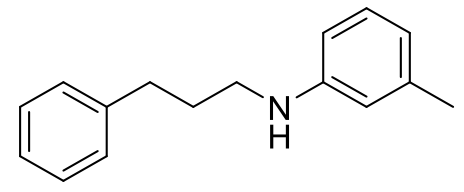

3-Methyl- $N$-(3-phenylpropyl)aniline (22): The general procedure (II) was followed using sodium tert-butoxide $(0.75$ mmol, $72.1 \mathrm{mg}, 1.5$ equiv.), 2-di-tert-butylphosphino2',4',6'-triisopropylbiphenyl

('BuXPhos)

ligand (0.022 mmol, $9.4 \mathrm{mg}, 4.4 \mathrm{~mol} \%$ ), bis[cinnamyl palladium(II)chloride] (0.005 mmol, $2.9 \mathrm{mg}, 1.1 \mathrm{~mol} \%$ ), 3-bromotoluene (0.5 mmol, $86 \mathrm{mg}, 61 \mu \mathrm{L}, 1.0$ equiv.) and 3-phenylpropylamine ( $0.6 \mathrm{mmol}, 85 \mathrm{mg}, 89 \mu \mathrm{L}, 1.25$ equiv.) in $0.2 \mathrm{~mL} 2 \mathrm{wt} \% \mathrm{HPMC}$ solution. The reaction was quenched (by addition of sat. aq. sodium sulfate solution) after 40 min and submitted to work-up. The desired product was obtained in $90 \%$ isolated yield (101 mg) as a yellow oil. The spectral data are consistent with those reported in the literature (12). 
${ }^{1} \mathbf{H}$ NMR $\left(500 \mathrm{MHz}, \mathrm{CDCl}_{3}\right) \delta$ 7.32-7.28 (m, 2H), 7.22-7.19 (m, 3H), 7.08-7.05 (m, 1H), 6.5 (d, $J=7.5 \mathrm{~Hz}, 1 \mathrm{H}), 6.43-6.39$ (m, 2H), 3.87 (bs, 1H, NH), 3.15 (t, $J=7.0 \mathrm{~Hz}, 2 \mathrm{H}$ ), $2.74(\mathrm{t}, J=7.5 \mathrm{~Hz}, 2 \mathrm{H}), 2.27$ (s, 3H), $1.96(\mathrm{dq}, J=8.8,7.2 \mathrm{~Hz}, 2 \mathrm{H}) \mathrm{ppm}$.

${ }^{13} \mathbf{C}$ NMR $\left(126 \mathrm{MHz}, \mathrm{CDCl}_{3}\right) \delta 148.4,141.7,138.9,129.1,128.4,125.9,118.1,113.5$, $109.9,43.4,33.4,31.1,21.6 \mathrm{ppm}$.

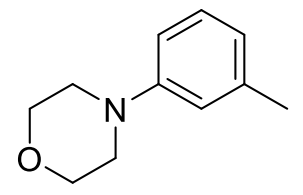

4-(m-Tolyl)morpholine (23): The general procedure (II) was followed using sodium tert-butoxide (1.5 mmol, $144 \mathrm{mg}, 1.5$ equiv.), 2-di-tertbutylphosphino-2',4',6'-triisopropylbiphenyl ('BuXPhos) ligand (0.044 mmol, $18.7 \mathrm{mg}, 4.4 \mathrm{~mol} \%$ ), bis[cinnamyl palladium(II)chloride] (0.011 mmol, 5.7 $\mathrm{mg}, 1.1 \mathrm{~mol} \%$ ), 3-bromotoluene ( $1.0 \mathrm{mmol}, 171 \mathrm{mg}, 121 \mu \mathrm{L}, 1.0$ equiv.) and morpholine (1.25 mmol, $109 \mathrm{mg}, 108 \mu \mathrm{L}, 1.25$ equiv.) in $0.2 \mathrm{~mL} 2 \mathrm{wt} \% \mathrm{HPMC}$ solution. The reaction was quenched after 5 min through the addition of sat. aq. sodium sulfate solution (same amount as HPMC/water solution) and the reaction mixture was stirred at $50^{\circ} \mathrm{C}$ for $5 \mathrm{~min}$. The precipitate was then filtered off and washed with EtOAc (5x). The filtrate was concentrated in vacuo and the crude was then purified by automated by automated flash column chromatography (gradiently $0-100 \%$ EtOAc in heptane). The desired product was obtained in $91 \%$ isolated yield $(161 \mathrm{mg})$ as a yellow oil. The spectral data are consistent with those reported in the literature (13).

${ }^{1} \mathbf{H}$ NMR $\left(500 \mathrm{MHz}, \mathrm{CDCl}_{3}\right) \delta 7.17(\mathrm{t}, J=7.5 \mathrm{~Hz}, 1 \mathrm{H}), 6.79-6.46(\mathrm{~m}, 3 \mathrm{H}), 3.86(\mathrm{t}, J=4.8$ $\mathrm{Hz}, 4 \mathrm{H}), 3.15$ (t, $J=4.8 \mathrm{~Hz}, 4 \mathrm{H}), 2.33$ (s, 3H) ppm.

${ }^{13} \mathbf{C}$ NMR $\left(126 \mathrm{MHz}, \mathrm{CDCl}_{3}\right) \delta 151.4,138.9,129.0,120.9,116.6,112.9,67.0,49.5$, $21.8 \mathrm{ppm}$.

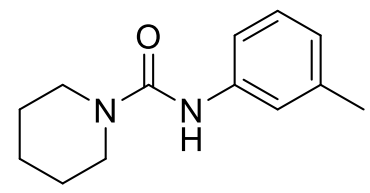

$N$-(m-tolyl)piperidine-1-carboxamide (24): The general procedure (II) was followed using sodium tert-butoxide (1.5 mmol, $144 \mathrm{mg}, \quad 1.5$ equiv.), 2-di-tert-Butylphosphino-2',4',6'triisopropylbiphenyl ( ${ }^{t}$ BuXPhos) ligand (0.044 mmol, $\left.18.7 \mathrm{mg}, 4.4 \mathrm{~mol} \%\right)$, bis[cinnamyl palladium(II)chloride] ( $0.011 \mathrm{mmol}, 5.7 \mathrm{mg}, 1.1 \mathrm{~mol} \%)$, 3-bromotoluene (1.0 mmol, 171 $\mathrm{mg}, 121 \mu \mathrm{L}, 1.0$ equiv.) and piperidine-1-carboxylic acid amide (1.25 mmol, $160 \mathrm{mg}, 1.25$ 
equiv.) in $0.2 \mathrm{~mL} 2 \mathrm{wt} \%$ HPMC solution. The reaction was quenched after $5 \mathrm{~min}$ through the addition of sat. aq. sodium sulfate solution (same amount as HPMC/water solution) and the reaction mixture was stirred at $50{ }^{\circ} \mathrm{C}$ for $5 \mathrm{~min}$. The precipitate was then filtered off and washed with EtOAc (5x). The filtrate was concentrated in vacuo and the crude was then purified by automated by automated flash column chromatography (0-100\% EtOAc in heptane). The desired product was obtained in $85 \%$ isolated yield $(186.5 \mathrm{mg})$ as a white solid.

${ }^{1} \mathbf{H}$ NMR (500 MHz, CDCl $)$ ) $8.24(\mathrm{~s}, 1 \mathrm{H}), 7.17-7.08(\mathrm{~m}, 2 \mathrm{H}), 6.86-6.79(\mathrm{~m}, 1 \mathrm{H}), 6.37(\mathrm{~s}$, 1H, NH), 3.53-3.31 (m, 4H), 2.31 (s, 3H), 1.77-1.47 (m, 6H) ppm.

${ }^{13}$ C NMR (126 MHz, $\left.\mathbf{C D C l}_{3}\right) \delta 155.0,139.2,138.7,128.6,123.6,120.5,116.8,45.3,25.7$, 24.4, $21.5 \mathrm{ppm}$.

HRMS (ESI) $\mathrm{m} / \mathrm{z}$ calcd. for $\mathrm{C}_{13} \mathrm{H}_{19} \mathrm{~N}_{2} \mathrm{O}\left([\mathrm{M}+\mathrm{H}]^{+}\right)$219.1492, found 219.1497 .

IR (film) vmax 3293, 2930, 2854, 2360, 1633, 1589, 1540, 1424, 1346, 1291, 1249, 1227, $1167,1023,982,860,785,751,691 \mathrm{~cm}^{-1}$.

\section{Melting point $121.7^{\circ} \mathrm{C}$}

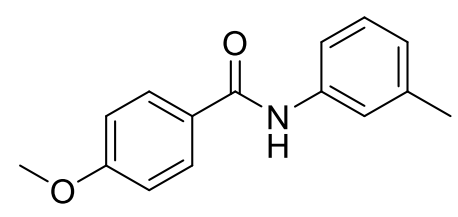

ligand $(0.022 \mathrm{mmol}$, 4-Methoxy- $N$-(m-tolyl)benzamide (25): The general procedure (I) was followed using sodium tert-butoxide $(0.75$ mmol, $72.5 \mathrm{mg}, 1.5$ equiv.), 2-di-tert-butylphosphino2', 4', 6'-triisopropylbiphenyl

( $\left.{ }^{t} \mathrm{BuXPhos}\right)$ palladium(II)chloride] (0.005 mmol, $2.9 \mathrm{mg}, 1.1 \mathrm{~mol} \%)$, 3-bromotoluene (0.5 mmol, $86 \mathrm{mg}, 61 \mu \mathrm{L}, 1.0$ equiv.) and 4-methoxybenzamide ( $0.6 \mathrm{mmol}, 95 \mathrm{mg}, 1.25$ equiv.). The reaction was quenched (by addition of sat. aq. sodium sulfate solution) after $30 \mathrm{~min}$ and submitted to work-up. The desired product was obtained in $99 \%$ isolated yield (120 mg) as a pale-yellow solid. The spectral data are consistent with those reported in the literature (12).

${ }^{1} \mathbf{H}$ NMR $\left(600 \mathrm{MHz}, \mathrm{CDCl}_{3}\right) \delta$ 7.87-7.80 (m, 2H), $7.69(\mathrm{~s}, 1 \mathrm{H}, \mathrm{NH}), 7.50(\mathrm{~s}, 1 \mathrm{H}), 7.40(\mathrm{~d}$, $J=8.0 \mathrm{~Hz}, 1 \mathrm{H}), 7.29-7.19$ (m, 1H), 7.00-6.93 (m, 3H), 3.88 (s, 3H), 2.37 (s, 3H) ppm. 
${ }^{13}$ C NMR (126 MHz, $\left.\mathrm{CDCl}_{3}\right) \delta 165.2,162.4,138.9,138.0,128.9,128.8,127.2,125.1$, $120.8,117.3,113.9,55.5,21.5 \mathrm{ppm}$.

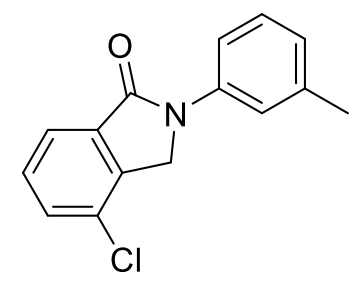

bromotoluene ( $0.5 \mathrm{mmol}, 86 \mathrm{mg}, 61 \mu \mathrm{L}, 1.0$ equiv.) and 4-chloroisoindolin-1one ( $0.6 \mathrm{mmol}, 105 \mathrm{mg}, 1.25$ equiv.). The reaction was quenched after 30 minutes with sat. aq. sodium sulfate solution and ethyl acetate, the mixture was filtered through a DryDisk separation membrane with GORE Microfiltration Media (Horizon technology, Lot\# B02400833323, Qty 50,65 mm). Upon filtration, only the organic phase passes through the filter. The filtrate was then concentrated in vacuo and purified by flash column chromatography on silica gel $(0-100 \%$ ethyl acetate/heptane). The desired product was obtained in $91 \%$ isolated yield $(117 \mathrm{mg})$ as a white solid.

${ }^{1} \mathbf{H}$ NMR $\left(500 \mathrm{MHz}, \mathrm{CDCl}_{3}\right) \delta 7.85(\mathrm{dd}, J=7.5,0.9 \mathrm{~Hz}, 1 \mathrm{H}), 7.72(\mathrm{~s}, 1 \mathrm{H}), 7.67(\mathrm{dd}, J=8.1$, $2.2 \mathrm{~Hz}, 1 \mathrm{H}), 7.58$ (dd, $J=7.9,0.9 \mathrm{~Hz}, 1 \mathrm{H}), 7.50$ (t, $J=7.4 \mathrm{~Hz}, 1 \mathrm{H}), 7.34$ (t, $J=7.9 \mathrm{~Hz}$, 1H), $7.07-7.02$ (m, 1H), 4.84 (s, 2H), 2.44 (s, 3H) ppm.

${ }^{13}$ C NMR $\left(126 \mathrm{MHz}, \mathrm{CDCl}_{3}\right) \delta 166.4,139.2,139.0,138.4,135.3,131.9,130.1,129.1$, $125.7,122.5,120.4,116.8,49.9,21.7 \mathrm{ppm}$.

HRMS (ESI) $\mathrm{m} / \mathrm{z}$ calcd. for $\mathrm{C}_{15} \mathrm{H}_{13} \mathrm{ClNO}\left([\mathrm{M}+\mathrm{H}]^{+}\right)$258.0680, found 258.0685 .

IR (film) vmax 1693, 1606, 1450, 1373, 1336, 1263, 1154, 850, 784, 746, $688 \mathrm{~cm}^{-1}$.

Melting point $126.9^{\circ} \mathrm{C}$

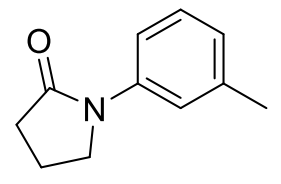

1-(m-Tolyl)pyrrolidin-2-one (27): The general procedure (I) was followed using sodium tert-butoxide $(0.75 \mathrm{mmol}, 73.0 \mathrm{mg}, 1.5 \mathrm{equiv}$.), 2-di-tert-butylphosphino-2',4',6'-triisopropylbiphenyl

( $\left.{ }^{t} \mathrm{BuXPhos}\right)$ ligand (0.022 mmol, $9.4 \mathrm{mg}, 4.4 \mathrm{~mol} \%$ ), bis[cinnamyl palladium(II)chloride] (0.005 mmol, $2.9 \mathrm{mg}, 1.1 \mathrm{~mol} \%)$, 3-bromotoluene ( $0.5 \mathrm{mmol}, 86 \mathrm{mg}, 61 \mu \mathrm{L}, 1.0$ equiv.) and 2- 
pyrrolidone ( $0.6 \mathrm{mmol}, 54 \mathrm{mg}, 48 \mu \mathrm{L}, 1.25$ equiv.). The reaction was quenched (by addition of sat. aq. sodium sulfate solution) after 5 minutes and submitted to work-up. The desired product was obtained in $97 \%$ isolated yield $(86 \mathrm{mg})$ as white crystals. The spectral data are consistent with those reported in the literature (14).

${ }^{1} \mathbf{H}$ NMR $\left(500 \mathrm{MHz}, \mathrm{CDCl}_{3}\right) \delta 7.45(\mathrm{~s}, 1 \mathrm{H}), 7.40-7.34(\mathrm{~m}, 1 \mathrm{H}), 7.26-7.22(\mathrm{~m}, 1 \mathrm{H})$, $6.96(\mathrm{ddt}, J=7.6,1.8,0.9 \mathrm{~Hz}, 1 \mathrm{H}), 3.85$ (t, $J=7.4 \mathrm{~Hz}, 2 \mathrm{H}), 2.60(\mathrm{t}, J=8.0 \mathrm{~Hz}, 2 \mathrm{H}), 2.36$ (s, $3 \mathrm{H}), 2.22-2.04(\mathrm{~m}, 2 \mathrm{H}) \mathrm{ppm}$.

${ }^{13}$ C NMR $\left(126 \mathrm{MHz} \mathrm{CDCl}_{3}\right) \delta 174.1,139.4,138.6,128.6,125.4,120.8,117.2,48.9,32.8$, 21.6, $18.1 \mathrm{ppm}$.

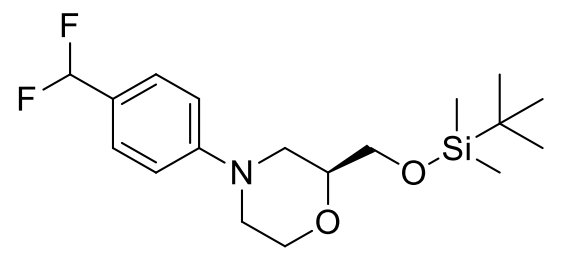

(S)-2-((tert-butyldimethylsilyl)oxy)methyl)-4-(4(difluoromethyl)phenyl)morpholine (28): $\quad$ The general procedure (II) was followed using [(cinnamyl)PdCl $]_{2}(2.90 \mathrm{mg}, 5.5 \mu \mathrm{mol}, 0.011$ equiv.),

${ }^{t} \mathrm{BuXPhos}$ (9.3 mg, $0.022 \mathrm{mmol}, 0.044$ equiv.), $\mathrm{NaO}{ }^{t} \mathrm{Bu}$ (72 mg, $0.75 \mathrm{mmol}, 1.5$ equiv.), 1 bromo-4-(difluoromethyl)benzene (109 mg, $0.5 \mathrm{mmol}, 1.0$ equiv.) and (S)-2-((tertbutyldimethylsilyl)oxy)methyl)morpholine (145 mg, $0.625 \mathrm{mmol}, \quad 1.25$ equiv.) in $0.2 \mathrm{~mL} 2 \mathrm{wt} \%$ HPMC solution. The reaction was quenched (by addition of sat. aq. sodium sulfate solution) after 5 min and submitted to work-up. The column was eluted with 0 $15 \%$ EtOAc in heptane $(18 \mathrm{~mL} / \mathrm{min})$. The product was obtained in $77 \%$ yield $(137 \mathrm{mg})$ as a clear oil.

${ }^{1}$ H NMR $\left(500 \mathrm{MHz}, \mathrm{CDCl}_{3}\right) \delta$ 7.45-7.37 (m, 2H), 6.98-6.90 (m, 2H), $6.58(\mathrm{t}, J=56.9 \mathrm{~Hz}$, $1 \mathrm{H}), 4.07-3.98(\mathrm{~m}, 1 \mathrm{H}), 3.82-3.60(\mathrm{~m}, 5 \mathrm{H}), 3.52-3.44(\mathrm{~m}, 1 \mathrm{H}), 2.90(\mathrm{td}, J=11.8 \mathrm{~Hz}$, $3.5 \mathrm{~Hz}, 1 \mathrm{H}), 2.63(\mathrm{dd}, J=12.2 \mathrm{~Hz}, 10.5 \mathrm{~Hz}, 1 \mathrm{H}), 0.92$ (s, 9H), 0.11-0.08 (m, 6H) ppm.

${ }^{13}$ C NMR $\left(126 \mathrm{MHz} \mathrm{CDCl}_{3}\right) \delta 153.0,127.0,126.9,126.9,125.5,125.3,125.1,117.1$, $115.3,115.0,113.4,76.1,66.6,64.5,51.0,48.3,26.1,18.5,-5.1,-5.2 \mathrm{ppm}$.

${ }^{19}$ F NMR (471 MHz, CDCl3) $\delta-108.08(\mathrm{~d}, J=56.9 \mathrm{~Hz}, 2 \mathrm{~F}) \mathrm{ppm}$.

IR (film) $v \max 1655,1589,1557,1518,1368,1221,1169,1079,816,656 \mathrm{~cm}^{-1}$. 


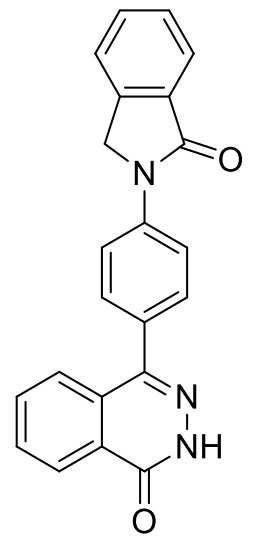

4-(4-(1-Oxoidoindolin-2-yl)phenyl)phthalazin-1(2H)-one (29): The general procedure (II) was followed using 4-(4-bromophenyl)phthalazin1(2H)-one (150 mg, $0.5 \mathrm{mmol}, \quad 1.0$ equiv.), isoindolin-1-one ( $80 \mathrm{mg}$, $0.6 \mathrm{mmol}, \quad 1.2$ equiv. $), \quad[(\text { cinnamyl }) \mathrm{PdCl}]_{2}(2.9 \mathrm{mg}, \quad 5.5 \mu \mathrm{mol}$, 0.011 equiv.), cBRIDP $(9.4 \mathrm{mg}, \quad 0.022 \mathrm{mmol}, \quad 0.044$ equiv.) and $\mathrm{KO}^{\prime} \mathrm{Bu}(169 \mathrm{mg}, 1.5 \mathrm{mmol} 3.0$ equiv.) in $0.4 \mathrm{~mL} 2 \mathrm{wt} \%$ HPMC solution. The reaction was quenched (by addition of sat. aq. sodium sulfate solution) after $12 \mathrm{~h}$ and submitted to work-up. The purification was perfomed by using a CombiFlash ${ }^{\circledR}$ system by $I S C O, 0-100 \% \mathrm{MeOH}$ in DCM. The product was obtained in $96 \%$ yield $(170 \mathrm{mg})$ as a brown solid.

${ }^{1} \mathbf{H}$ NMR $\left(500 \mathrm{MHz}, \mathrm{DMSO}-d_{6}\right) \delta 12.85(\mathrm{~s}, 1 \mathrm{H}), 8.41-8.30(\mathrm{~m}, 1 \mathrm{H}), 8.19-8.07(\mathrm{~m}, 2 \mathrm{H})$, 7.98-7.87 (m, 2H), 7.86-7.80 (m, 1H), 7.79-7.75 (m, 1H), 7.74-7.63 (m, 4H), 7.62$7.52(\mathrm{~m}, 1 \mathrm{H}), 5.15(\mathrm{~s}, 2 \mathrm{H}) \mathrm{ppm}$.

${ }^{13}$ C NMR (126 MHz, DMSO- $\left.d_{6}\right) \delta 166.9,159.2,145.9,141.1,140.0,133.6,132.5,132.3$, 131.6, 130.6, 130.0, 129.0, 127.9, 126.6, 126.1, 123.4, 123.4, 118.9, 50.4 ppm.

HRMS (ESI) $\mathrm{m} / \mathrm{z}$ calcd. for $\mathrm{C}_{22} \mathrm{H}_{16} \mathrm{~N}_{3} \mathrm{O}_{2}\left([\mathrm{M}+\mathrm{H}]^{+}\right) 354.1237$, found 354.1235 .

IR (film) vmax 1668, 1519, 1375, 1303, 1219, 1149, 835, 794, 729, 682, $549 \mathrm{~cm}^{-1}$.

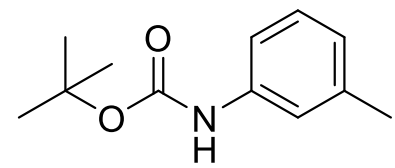

$N$-(m-tolyl)pivalamide (30): The general procedure (II) was followed using sodium tert-butoxide $(0.75 \mathrm{mmol}, 72.5 \mathrm{mg}$, 1.5 equiv.), 2-di-tert-butylphosphino-2',4',6'-triisopropyl biphenyl ('BuXPhos) ligand (0.022 mmol, $9.4 \mathrm{mg}, 4.4 \mathrm{~mol} \%)$, bis[cinnamyl palladium(II)chloride] (0.005 mmol, $2.9 \mathrm{mg}, 1.1 \mathrm{~mol} \%)$, 3-bromotoluene $(0.5 \mathrm{mmol}$, $86 \mathrm{mg}, 61 \mu \mathrm{L}, 1.0$ equiv.) and $t$-butyl carbamate ( $0.6 \mathrm{mmol}, 74 \mathrm{mg}, 1.25$ equiv.) in $0.2 \mathrm{~mL}$ $2 \mathrm{wt} \%$ HPMC solution. The reaction was quenched after $5 \mathrm{~min}$ through the addition of sat. aq. sodium sulfate solution (same amount as HPMC/water solution) and the reaction mixture was stirred at $50{ }^{\circ} \mathrm{C}$ for $5 \mathrm{~min}$. The precipitate was then filtered off and washed with EtOAc (5x). The filtrate was concentrated in vacuo and the crude was then purified by automated by automated flash column chromatography (gradiently $0-100 \%$ EtOAc in 
heptane). The desired product was obtained in $96 \%$ isolated yield $(100 \mathrm{mg})$ as a white solid. The spectral data are consistent with those reported in the literature (15).

${ }^{1}$ H NMR $\left(500 \mathrm{MHz}, \mathrm{CDCl}_{3}\right) \delta 7.26$ (brs, $\left.1 \mathrm{H}\right), 7.16$ (t, $\left.J=7.8 \mathrm{~Hz}, 1 \mathrm{H}\right), 7.12-7.06(\mathrm{~m}, 1 \mathrm{H})$, $6.85(\mathrm{~d}, J=7.5 \mathrm{~Hz}, 1 \mathrm{H}), 6.45(\mathrm{~s}, 1 \mathrm{H}, \mathrm{NH}), 2.33(\mathrm{~s}, 3 \mathrm{H}), 1.52(\mathrm{~s}, 9 \mathrm{H})$.

${ }^{13}$ C NMR (126 MHz, $\left.\mathrm{CDCl}_{3}\right) \delta 152.8,138.9,138.3,128.8,123.8,119.1,115.6,80.4,28.4$, 21.5.

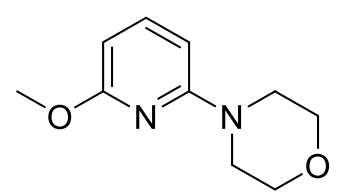

4-(6-Methoxypyridin-2-yl)morpholine (31): The general procedure (II) was followed and the desired product was obtained as a colorless liquid, $89 \%$ yield (42 $\mathrm{mg}), \mathrm{R}_{f}=0.3$ (9:1, hexanes/ethyl acetate). The spectral data are consistent with those reported in the literature (16).

${ }^{1} \mathbf{H}$ NMR $\left(400 \mathrm{MHz}, \mathrm{CDCl}_{3}\right) \delta 7.41(\mathrm{t}, J=7.9 \mathrm{~Hz}, 1 \mathrm{H}), 6.24-5.98(\mathrm{~m}, 2 \mathrm{H}), 3.86(\mathrm{~s}, 3 \mathrm{H})$, $3.82(\mathrm{t}, J=5.2 \mathrm{~Hz}, 4 \mathrm{H}), 3.48(\mathrm{t}, J=4.4 \mathrm{~Hz}, 4 \mathrm{H}) \mathrm{ppm}$.

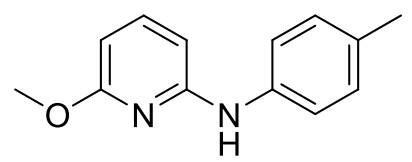

6-Methoxy- $N$-(p-tolyl)pyridin-2-amine (32): The general procedure (II) was followed and the desired product was obtained as a colorless liquid, $91 \%$ yield (48.2 $\mathrm{mg})(91 \%)$, $\mathrm{R}_{f}=0.4$ (4:1, hexanes/ethyl acetate). The spectral data are consistent with those reported in the literature (17).

${ }^{1} \mathbf{H}$ NMR $\left(400 \mathrm{MHz}, \mathrm{CDCl}_{3}\right) \delta 7.68(\mathrm{t}, J=7.9 \mathrm{~Hz}, 1 \mathrm{H}), 7.55(\mathrm{~d}, J=8.4 \mathrm{~Hz}, 2 \mathrm{H}), 7.43(\mathrm{~d}$, $J=8.1 \mathrm{~Hz}, 2 \mathrm{H}), 6.64(\mathrm{~d}, J=7.9 \mathrm{~Hz}, 1 \mathrm{H}), 6.60(\mathrm{~s}, 1 \mathrm{H}), 6.47$ (d, $J=8 \mathrm{~Hz}, 1 \mathrm{H}), 4.20(\mathrm{~s}, 3 \mathrm{H})$, $2.63(\mathrm{~s}, 3 \mathrm{H}) \mathrm{ppm}$.

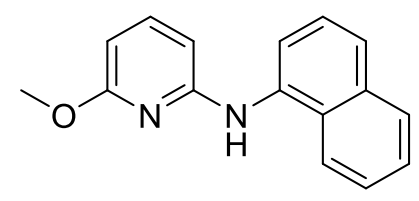

6-Methoxy- $N$-(naphthalen-1-yl)pyridin-2-amine (33): The general procedure (II) was followed and the desired product was obtained as an oil, $90 \%$ yield (56.2 mg), $\mathrm{R}_{f}=0.4 \quad(4: 1$, hexanes/ethyl acetate).

${ }^{1} \mathbf{H}$ NMR (400 MHz, $\left.\mathrm{CDCl}_{3}\right) \delta$ 8.19-7.97 (m, 1H), $7.89(\mathrm{~m}, 1 \mathrm{H}), 7.69(\mathrm{~d}, J=8 \mathrm{~Hz}, 1 \mathrm{H})$, $7.62(\mathrm{~d}, J=7.4 \mathrm{~Hz}, 1 \mathrm{H}), 7.56-7.40(\mathrm{~m}, 3 \mathrm{H}), 7.35$ (t, $J=8 \mathrm{~Hz}, 1 \mathrm{H}), 6.61$ (s, 1H), 6.27-6.08 (m, 2H), $3.93(\mathrm{~s}, 3 \mathrm{H}) \mathrm{ppm}$. 
${ }^{13}$ C NMR (100 MHz, $\left.\mathrm{CDCl}_{3}\right) \delta 163.9,156.2,140.4,136.2,134.7,129.0,128.6,126.3$, $126.1,126.0,125.0,122.3,120.2,99.9,99.3,53.5 \mathrm{ppm}$.

IR $v=3384(\mathrm{~m}), 3049(\mathrm{~m}), 2944(\mathrm{~m}), 1572(\mathrm{~s}) \mathrm{cm}^{-1}$.

HRMS (ESI) $\mathrm{m} / \mathrm{z}$ calcd. for $\mathrm{C}_{16} \mathrm{H}_{15} \mathrm{~N}_{2} \mathrm{O}\left([\mathrm{M}+\mathrm{H}]^{+}\right)$251.1179, found 251.1179 .

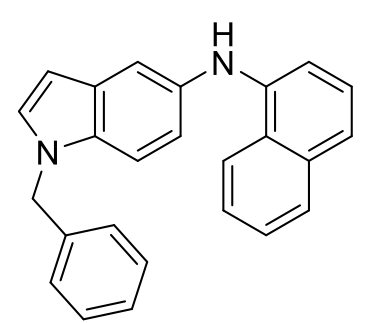

1-Benzyl- $N$-(naphthalen-1-yl)-1H-indol-5-amine (34): The general procedure (II) was followed and the desired product was obtained as a light brown solid, $89 \%$ yield $(77 \mathrm{mg}), \mathrm{R}_{f}=0.2(4: 1$, hexanes/ethyl acetate).

${ }^{1}$ H NMR $\left(400 \mathrm{MHz}, \mathrm{CDCl}_{3}\right) \delta 8.10-8.01(\mathrm{~m}, 1 \mathrm{H}), 7.88-7.83(\mathrm{~m}$, 1H), 7.52-7.47 (m, 2H), 7.43-7.39 (m, 2H), 7.37-7.22 (m, 6H), 7.16 (t, $J=5.3 \mathrm{~Hz}, 2 \mathrm{H})$, $7.12(\mathrm{~d}, J=7.5 \mathrm{~Hz}, 1 \mathrm{H}), 7.06(\mathrm{dd}, J=8.6,2.1 \mathrm{~Hz}, 1 \mathrm{H}), 6.48(\mathrm{~d}, J=3.1 \mathrm{~Hz}, 1 \mathrm{H}), 6.04$ (s, 1H), 5.35 (s, 2H) ppm.

${ }^{13} \mathrm{C}$ NMR $\left(100 \mathrm{MHz}, \mathrm{CDCl}_{3}\right) \delta 142.1,137.6,136.0,134.7,133.3,129.6,129.1,128.9$, $128.7,127.8,126.9,126.4,126.0,125.6,125.3,121.1,120.2,117.8,113.1,110.8,110.6$, $101.4,50.4 \mathrm{ppm}$.

IR $v=3385(\mathrm{~m}), 2978(\mathrm{w}), 2947(\mathrm{~m}), 1570(\mathrm{~s}) \mathrm{cm}^{-1}$.

HRMS (ESI) $\mathrm{m} / \mathrm{z}$ calcd. for $\mathrm{C}_{25} \mathrm{H}_{21} \mathrm{~N}_{2}\left([\mathrm{M}+\mathrm{H}]^{+}\right) 349.1698$, found 349.1699 .

Melting point: $98^{\circ} \mathrm{C}$

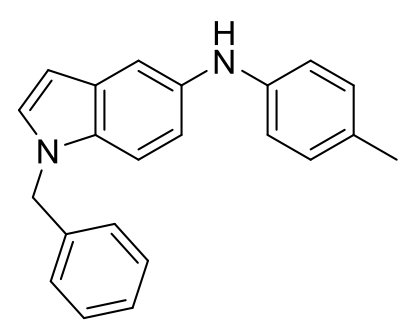

1-Benzyl- $N$-( $p$-tolyl)-1H-indol-5-amine (35): The general procedure (II) was followed and the desired product was obtained as a yellow liquid, $87 \%$ yield $(68 \mathrm{mg}), \mathrm{R}_{f}=0.2 \quad(4: 1$, hexanes/ethyl acetate).

${ }^{1} \mathbf{H}$ NMR $\left(400 \mathrm{MHz}, \mathrm{CDCl}_{3}\right) \delta 7.39(\mathrm{~s}, 1 \mathrm{H}), 7.36-7.24(\mathrm{~m}, 3 \mathrm{H})$, $7.20(\mathrm{~d}, J=8.6 \mathrm{~Hz}, 1 \mathrm{H}), 7.17-7.10(\mathrm{~m}, 3 \mathrm{H}), 7.04$ (d, $J=8 \mathrm{~Hz}, 2 \mathrm{H}), 6.97$ (d, $J=8.6 \mathrm{~Hz}$, $1 \mathrm{H}), 6.89(\mathrm{~d}, J=8 \mathrm{~Hz}, 2 \mathrm{H}), 6.46(\mathrm{~d}, J=2 \mathrm{~Hz}, 1 \mathrm{H}), 5.51(\mathrm{~s}, 1 \mathrm{H}), 5.31$ (s, 2H), 2.29 (s, 3H) ppm. 
${ }^{13} \mathrm{C}$ NMR $\left(100 \mathrm{MHz}, \mathrm{CDCl}_{3}\right) \delta 143.6,137.7,136.0,133.1,129.8,129.5,129.0,128.9$, $128.8,127.7,126.9,117.3,116.3,112.1,110.4,101.3,50.4,20.7$ ppm.

IR $v=3389(\mathrm{~m}), 3028(\mathrm{~m}), 2917(\mathrm{~m}), 1612(\mathrm{~s}) \mathrm{cm}^{-1}$.

HRMS (ESI) $\mathrm{m} / \mathrm{z}$ calcd. for $\mathrm{C}_{22} \mathrm{H}_{21} \mathrm{~N}_{2}\left([\mathrm{M}+\mathrm{H}]^{+}\right) 313.1699$, found m/z 313.1699.

\section{General Procedure for Amide Couplings (III):}

The corresponding acid (1.1 equiv.) was weighed into a $5 \mathrm{~mL}$ microwave vial containing a magnetic stirring bar. $2 \mathrm{wt} \%$ HPMC solution (in degassed Millipore water) was added, followed by the addition of 2,6-lutidine (3.1 equiv.). The reaction mixture was vigorously stirred at room temperature under open air atmosphere until full solubilization of the acid (3-10 min). Initially the acid-HPMC mixture is milky white, which upon addition of 2,6lutidine and stirring turns into a clear solution. This process can be shortened significantly if the reaction mixture is sonicated for a few seconds. Subsequently, the corresponding amine (1.0 equiv.) followed by COMU (1.1 equiv.) were added. The reaction mixture turns yellow and a suspension is formed. The yellow color is an indicator for the release of the free-base form of the oxyme activating agent. The reaction mixture was stirred at room temperature until full consumption of the amine starting material was observed, monitored by TLC $\left(\mathrm{CH}_{2} \mathrm{Cl}_{2}: \mathrm{CH}_{3} \mathrm{OH}(5 \%), \mathrm{UV}\right.$ and/or $\mathrm{KMnO}_{4}$ and Schlitters reagent for staining) and/or LCMS.

Upon full consumption of the amine starting material, the reaction was quenched with a few drops of $\mathrm{CH}_{2} \mathrm{Cl}_{2}$ and sat. aq. $\mathrm{Na}_{2} \mathrm{SO}_{4}$ solution (same amount as the $2 \mathrm{wt} \% \mathrm{HPMC} /$ water solution). This was followed by the addition of EtOAc and the mixture was stirred at room temperature for $5 \mathrm{~min}$. HPMC crashes out once sat. aq. $\mathrm{Na}_{2} \mathrm{SO}_{4}$ solution is added. The precipitate was then filtered off and washed intensively with EtOAc. The filtrate was then washed with $1 \mathrm{M} \mathrm{HCl}(3 \mathrm{x})$ and sat. aq. $\mathrm{Na}_{2} \mathrm{CO}_{3}$ solution in water $(4 \mathrm{x}$; aq. layer turns yellow; washing with $\mathrm{Na}_{2} \mathrm{CO}_{3}$ solution until the aq. phase does not show any yellow color). The organic phase was dried over $\mathrm{MgSO}_{4}$, filtered and concentrated in vacuo. Almost all crude products were spectroscopically and analytically clean, therefore avoiding any additional purification. The reported yields refer to crude products. However, for a few examples an aliquot was purified by preparative SFC for comparative reasons. 

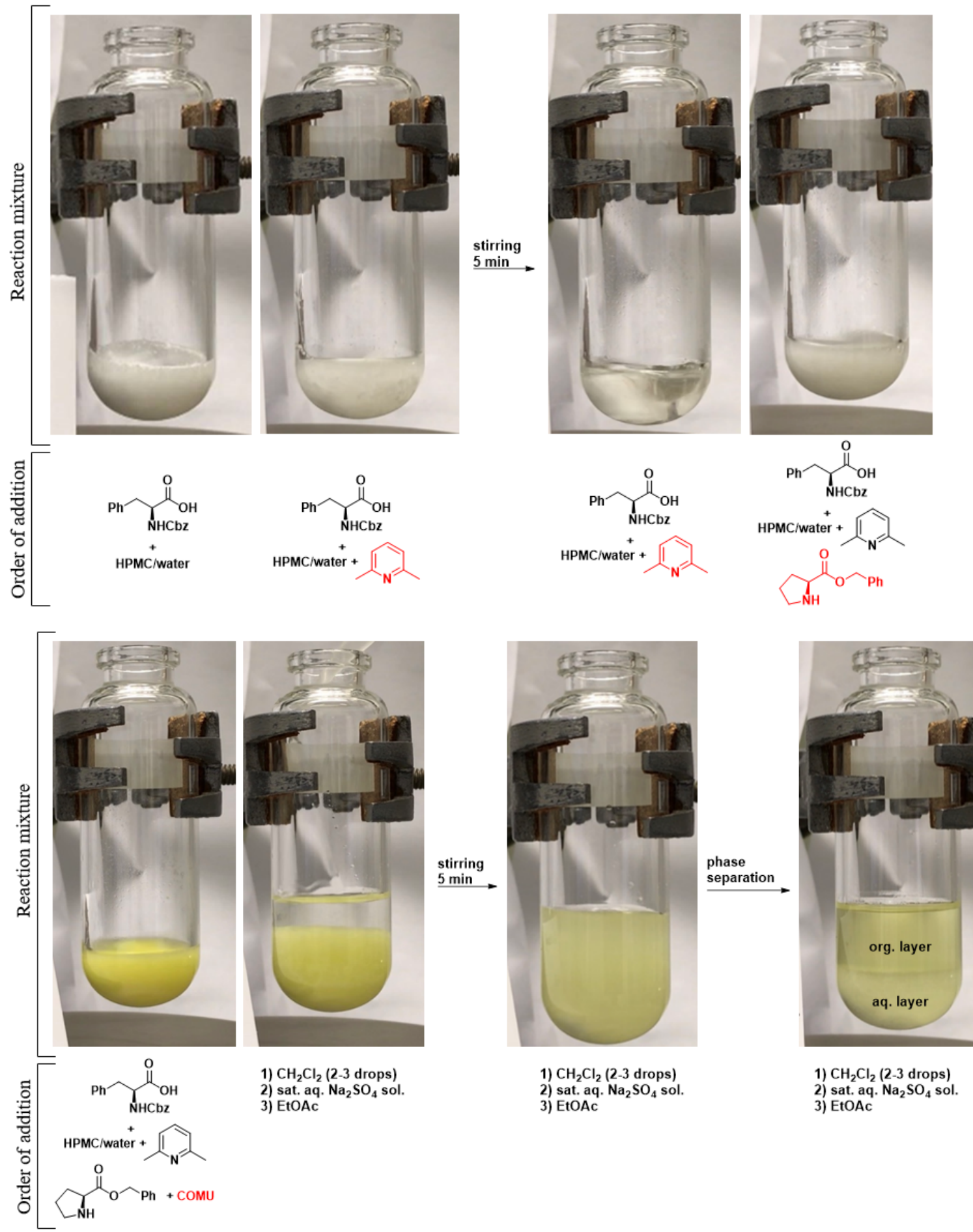

Figure S6. General procedure for the amide coupling in HPMC/water. 


\subsection{0-gram scale synthesis of compound 43:}

In $2 \mathrm{~L}$ round bottom flask containing a PTFE-coated magnetic stir bar, phenylacetic acid (100 g, 0.73 mol, 1.0 equiv.) was taken. Freshly prepared $0.1 \mathrm{wt} \%$ HPMC solution in water $(918 \mathrm{~mL})$ was added to the reaction mixture. Reaction mixture was stirred for 5 minutes at rt. 2,6-Lutidine (255 mL, $2.20 \mathrm{~mol}, 3.0$ equiv.) was added to the reaction mixture and mixture was stirred for two minutes at rt. Addition of 2,6-lutidine converts the reaction mixture into a clear solution. COMU (339 g, 97\% purity, 0.77 mol, 1.05 equiv.) and aryl piperazine (218 g, $0.73 \mathrm{~mol}, 1.0$ equiv.) were added to the reaction mixture. Reaction mixture was stirred for one minute at rt. After one minute, stirring was stopped and reaction mixture was cooled to $1{ }^{\circ} \mathrm{C}$. Cooling results in product precipitation. Solid product was filtered under vacuum filtration. Solid product was washed with $500 \mathrm{~mL}$ water and then with $1 \% \mathrm{Et}_{2} \mathrm{O}$ in cyclohexane $(200 \mathrm{~mL})$ to obtain pure product. The original aqueous filtrate was kept at $1{ }^{\circ} \mathrm{C}$ for 1 hour, which results in further product precipitation. Solid was

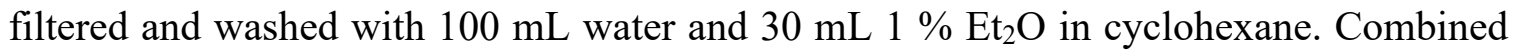
solids were dried under reduced pressure for $24 \mathrm{~h}$. Pure product was obtained as white solid, $307 \mathrm{~g},>99 \%$ yield.

\subsection{0-gram scale synthesis of compound 47:}

In $2 \mathrm{~L}$ round bottom flask containing a PTFE-coated magnetic stir bar, ((benzyloxy)carbonyl)-L-phenylalanine (100 g, 0.334 mol, 1.0 equiv.) was taken. Freshly prepared $0.1 \mathrm{wt} \%$ HPMC solution in water $(418 \mathrm{~mL})$ was added to the reaction mixture. Reaction mixture was stirred for 5 minutes at rt. 2,6-Lutidine $(116 \mathrm{~mL}, 1.002 \mathrm{~mol}$, 3.0 equiv.) was added to the reaction mixture and mixture was stirred for two minutes at rt. Addition of 2,6-lutidine converts the reaction mixture to clear solution. COMU (155 g, 97\% purity, $0.351 \mathrm{~mol}, 1.05$ equiv) and ethyl L-leucinate (53.2 g, 0.334 mol, 1.0 equiv.) were added to the reaction mixture. Reaction mixture was stirred for one minute at rt. After one minute, stirring was stopped and reaction mixture was cooled to $0{ }^{\circ} \mathrm{C}$ and $400 \mathrm{~mL} 3 \mathrm{M}$ cold $\mathrm{HCl}$ were added to the reaction mixture over the period of 10 minutes. Cooling and addition of $\mathrm{HCl}$ results in product precipitation. Solid product was filtered under vacuum filtration. Solid product was washed with additional $500 \mathrm{~mL}$ cold water Solid was dried under reduced pressure for $24 \mathrm{~h}$ at $45^{\circ} \mathrm{C}$. Pure product was obtained as white solid, 128.5 g, $96 \%$ yield. 


\section{Amide Couplings: Optimization Studies}

\subsection{Screening of different coupling reagents}

In order to evaluate different coupling reagents general procedure (III) was followed using a different purification method since not all by-products of the individual coupling reagents can be removed by aq. washing steps. Therefore, the reactions were quenched after $2 \mathrm{~h}$ using a few drops $\mathrm{CH}_{2} \mathrm{Cl}_{2}$, sat. aq. $\mathrm{Na}_{2} \mathrm{SO}_{4}$ solution and EtOAc and stirred for $5 \mathrm{~min}$ at $\mathrm{rt}$. The mixture was filtered and intensively washed with EtOAc. The filtrate was washed with $1 \mathrm{M} \mathrm{HCl}(3 \mathrm{x})$ and the org. layer was dried over $\mathrm{Na}_{2} \mathrm{SO}_{4}$ and concentrated under vacuo. The crude product was dissolved in $\mathrm{MeOH}(1.8 \mathrm{~mL})$ and submitted to preparative HPLC for purification (column: Waters Xselect CSH C18, 150x30mm, $5 \mu \mathrm{m}$; eluents: Millipore water $+0.1 \% \mathrm{FA}(\mathrm{A}), \mathrm{MeCN}+0.1 \% \mathrm{FA}(\mathrm{B})$; gradient: $45-60 \% \mathrm{~B}$ in $20 \mathrm{~min}, 50 \mathrm{~mL} / \mathrm{min}$ ).

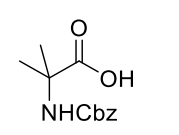

(1.1 equiv.)

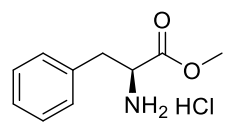

(1.0 equiv.)

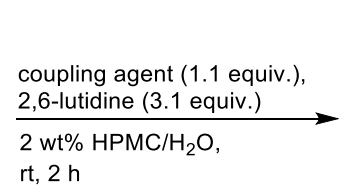

rt, 2 h

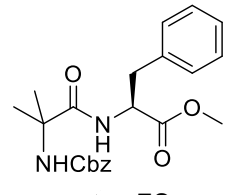

52

\begin{tabular}{cccc}
\hline Entry $^{\mathbf{a}}$ & Couping agent & Yield [\%] $^{\mathbf{b}}$ & ee [\%] \\
\hline 1 & COMU & 79 & $>99.9$ \\
2 & EDC $^{\mathrm{c}}$ & 84 & $>99.9$ \\
3 & EDC & 68 & $>99.9$ \\
4 & CDI & 0 & - \\
5 & T3P & \\
6 & CDMT & 11 & $>99.9$ \\
7 & DMT-MM & $0^{\mathrm{e}}$ & - \\
8 & TBTU & 78 & $>99.9$ \\
9 & PyBOP & 53 & $>99.9$ \\
10 & DMC & 37 & $>99.9$ \\
\hline
\end{tabular}

Table S2. Screening of different coupling agents. ${ }^{a}$ Reactions were performed on $0.5 \mathrm{mmol}$ scale at $0.8 \mathrm{M}$ concentration following the general procedure (I) for amide couplings. ${ }^{b}$ isolated yields, purification via HPLC for comparable results. ${ }^{\mathrm{c}}$ use of 1.1 equiv. HOBt as additive. ${ }^{\mathrm{d}}$ T3P as a solution in EtOAc (50 wt\%). ${ }^{e}$ only $31 \%$ of $\mathrm{S}_{\mathrm{N}} \mathrm{Ar}$ triazine side product observed. \%ee determined by chiral SFC. 


\subsection{Epimerization Study}

In order to investigate the occurrence of epimerization both stereoisomers of the dipeptides 51 and 52 were synthesized (Table S3) according to general procedure (III) with the additional purification described in section 9.1. A mixture of 51a and 51b as well as of 52a and 52b was prepared and analyzed by chiral SFC (column: Phenomenex LUX Cellulose2, 110x4.6 mm, $5 \mu$ m; eluents: $\mathrm{CO}_{2}(\mathrm{~A}), \mathrm{MeOH}+0.2 \% \mathrm{NH}_{4} \mathrm{OH}$ (B), gradient: $5-50 \% \mathrm{~B}$ in $3 \mathrm{~min}$, then isocratic at $50 \% \mathrm{~B}$ for $1 \mathrm{~min}, 3.5 \mathrm{~mL} / \mathrm{min}$ ) to obtain reference chromatograms for both mixtures (Fig. S7 and Fig. S10). Afterwards, the individual reaction products 51a-b and 52a-b were analyzed by chiral SFC (Fig. S8-S9 and Fig. S11-S12) to determine the enantiomeric excess (\% ee).

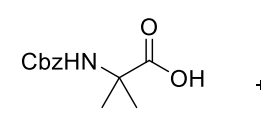

(1.1 equiv.)

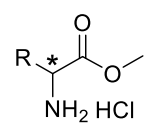

(1.0 equiv.)

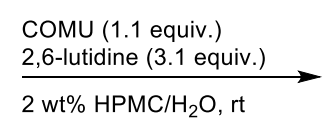

2 wt $\% \mathrm{HPMC} / \mathrm{H}_{2} \mathrm{O}$, rt

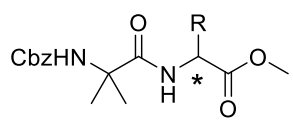

(S), $R=P h 51 a \quad(S), R=B n$ 52a

\begin{tabular}{ccccc}
\hline Entry $^{\mathbf{a}}$ & Amine & Product & Yield [\%] $^{\mathbf{b}}$ & ee [\%] \\
\hline 1 & L-Phe-OMe & Z-AIB-L-Phe-OMe (52a) & 75 & $>99.9$ \\
2 & D-Phe-OMe & Z-AIB-D-Phe-OMe (52b) & 81 & $>99.9$ \\
3 & L-PhG-OMe & Z-AIB-L-PhG-OMe (51a) & 80 & $>99.9$ \\
4 & D-PhG-OMe & Z-AIB-D-PhG-OMe (51b) & 76 & $>99.9$
\end{tabular}

Table S3. Epimerization study with COMU as coupling agent. ${ }^{\text {a }}$ Reactions were performed on $0.5 \mathrm{mmol}$ scale at $0.8 \mathrm{M}$ concentration following the general procedure (III) for amide couplings. ${ }^{b}$ isolated yields, purification via HPLC. ee determined by chiral SFC. 


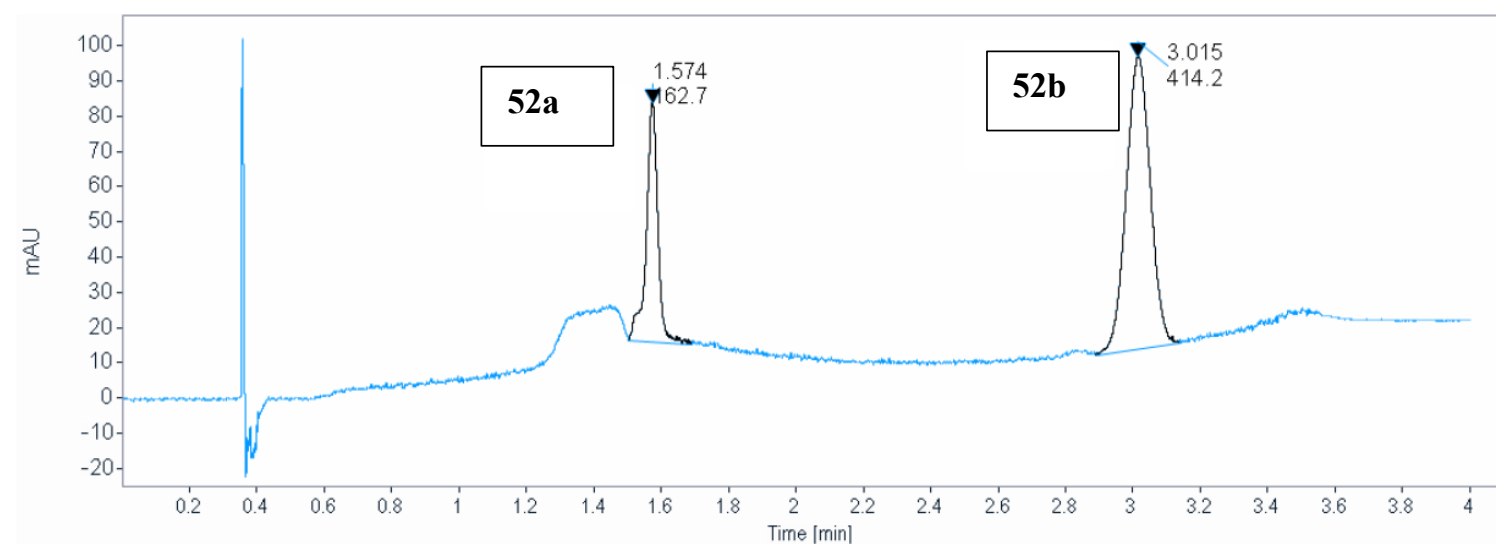

Figure S7. SFC chromatogram of a mixture of dipeptides 52a and 52b.

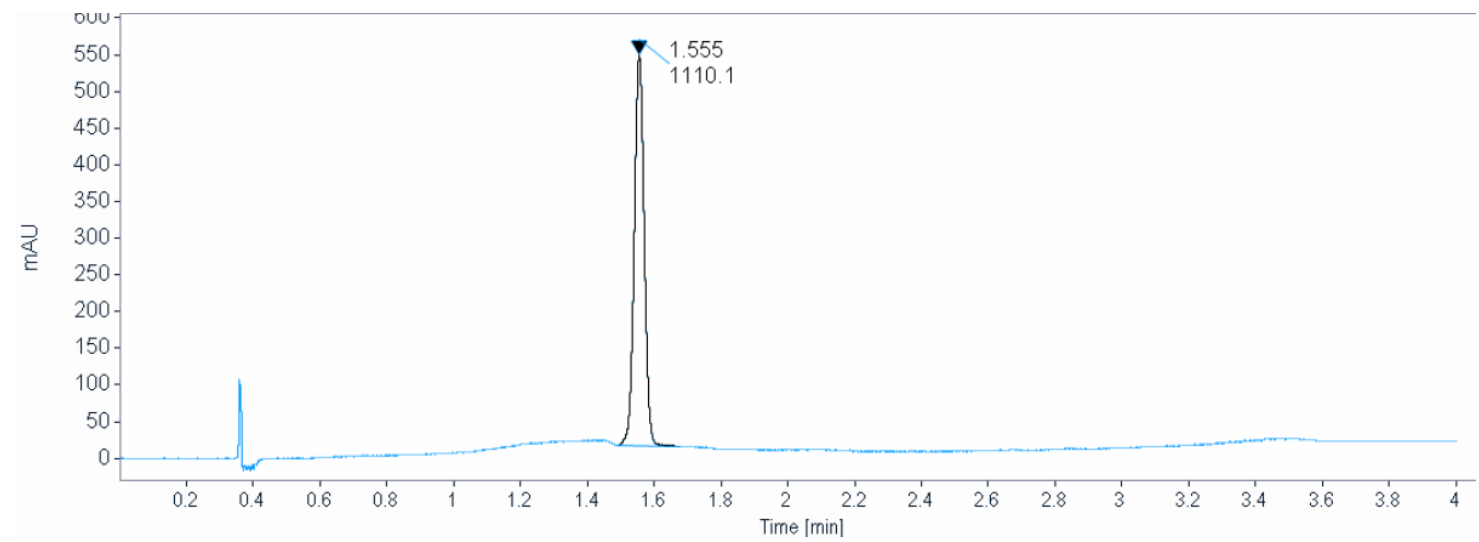

Figure S8. SFC chromatogram of dipeptide 52a.

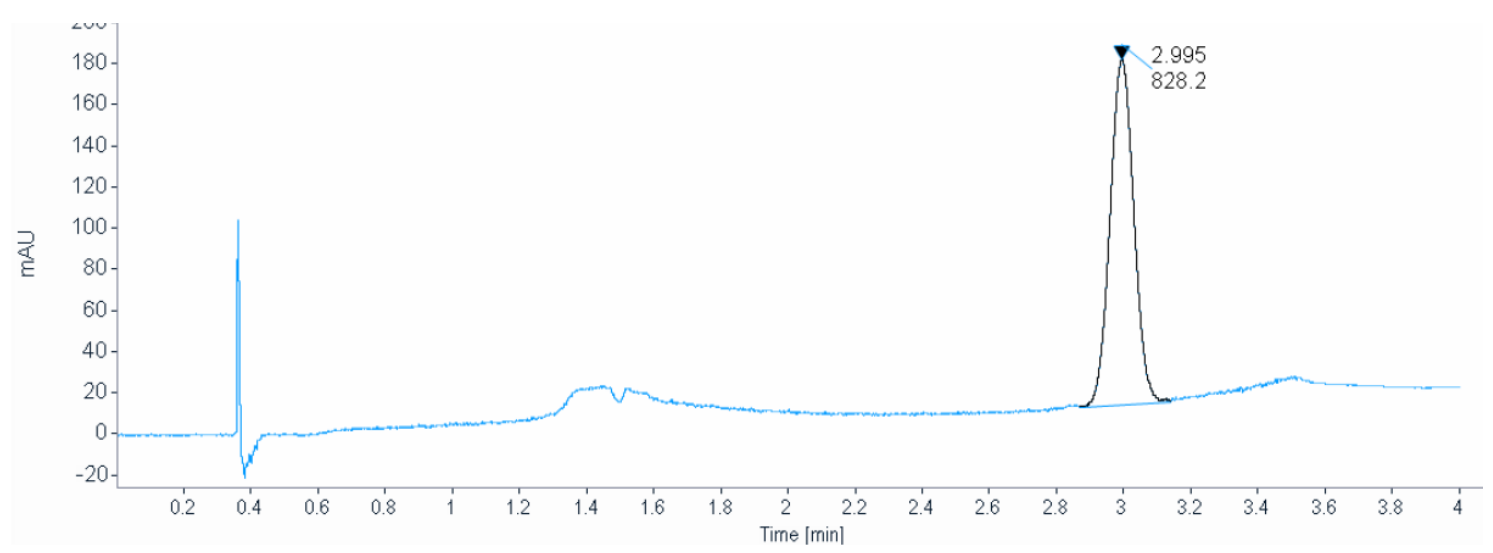

Figure S9. SFC chromatogram of dipeptide $52 \mathrm{~b}$. 


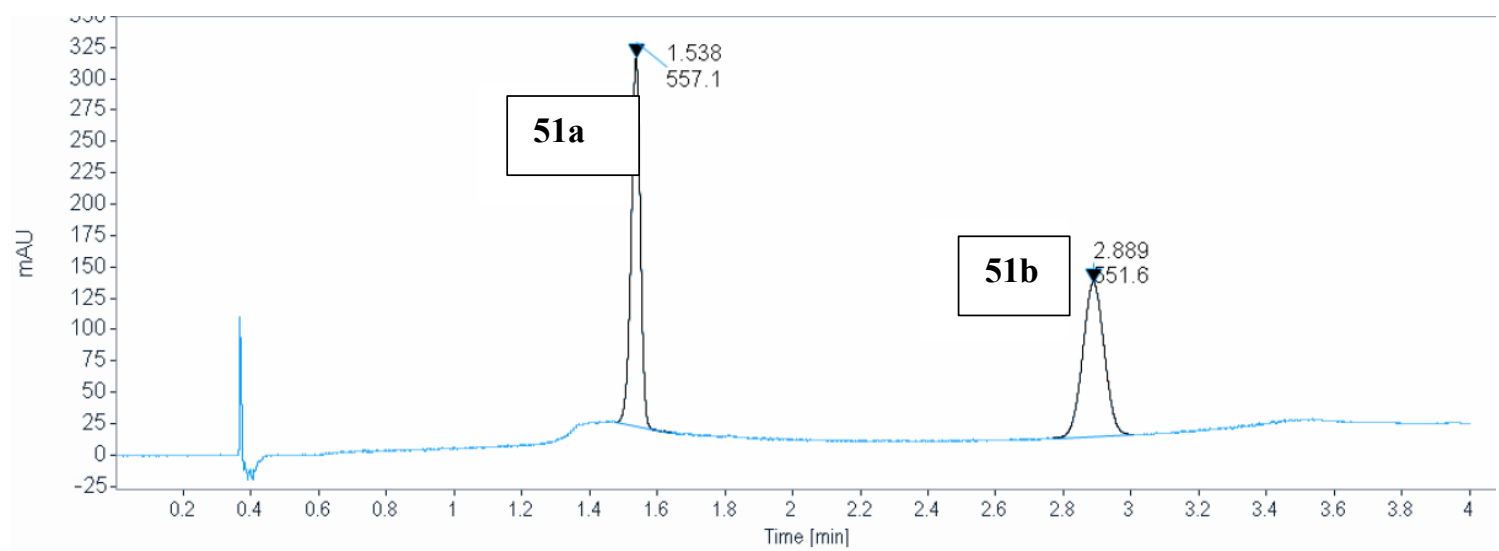

Figure S10. SFC chromatogram of a mixture of dipeptides 51a and 51b.

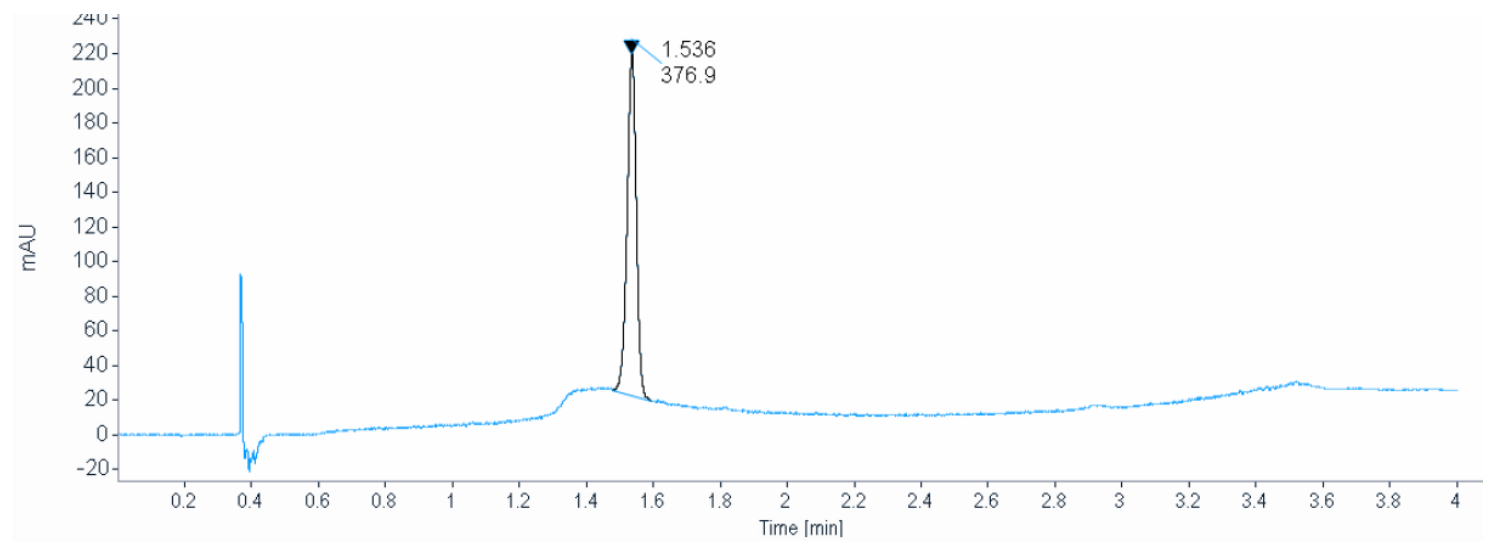

Figure S11. SFC chromatogram of dipeptide 51a.

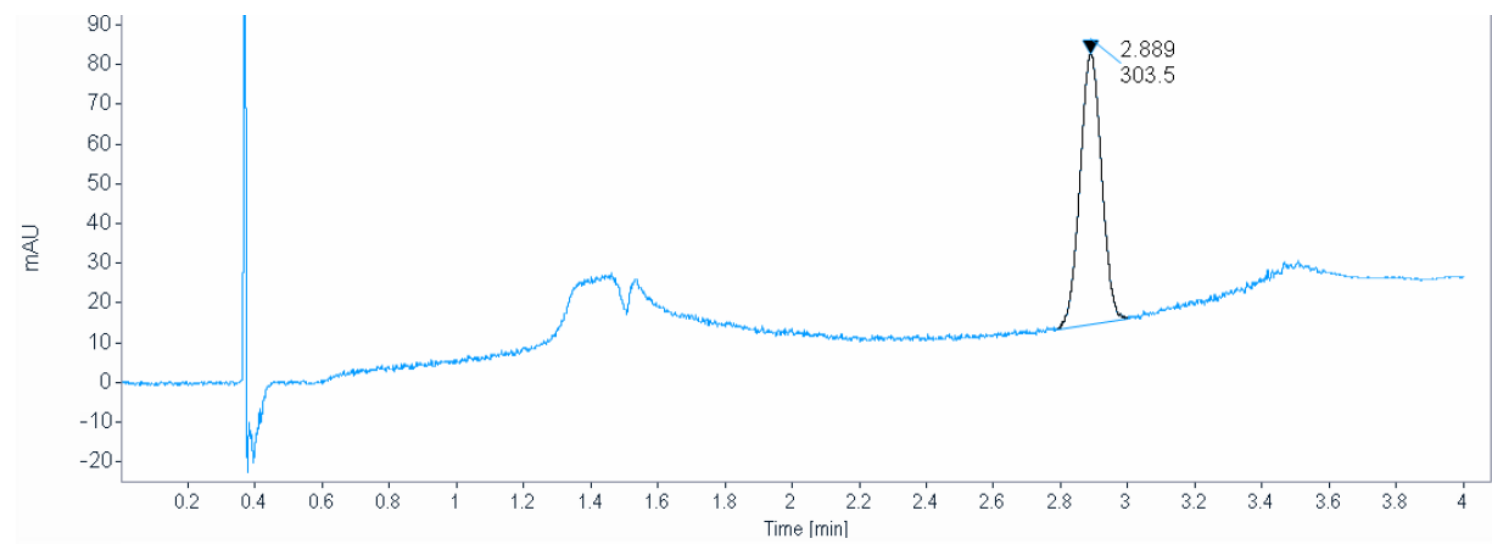

Figure S12. SFC chromatogram of dipeptide 51b. 


\subsection{Optimization of the reaction conditions for scale-up}

Optimization of the reaction conditions was performed for the purpose of utilizing this transformation on larger scale (up to grams). The experiments shown in Table S4 were performed on $1 \mathrm{mmol}$ scale following general procedure (III). The products were isolated using the extraction conditions described in general procedure. No further purification was necessary since the crude products were spectroscopically and analytically clean (unless otherwise stated).
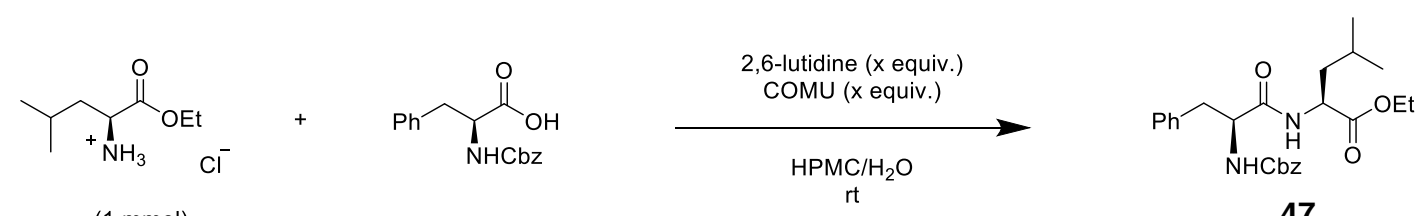

\begin{tabular}{lllllllll}
\hline \hline Entry & $\begin{array}{l}\text { Amine } \\
\text { [equiv.] }\end{array}$ & $\begin{array}{l}\text { Acid } \\
\text { [equiv.] }\end{array}$ & $\begin{array}{l}\text { Base } \\
\text { [equiv.] }\end{array}$ & $\begin{array}{l}\text { COMU } \\
\text { [equiv.] }\end{array}$ & Time $^{\text {a }}$ & $\begin{array}{l}\text { HPMC } \\
\text { [wt\%] }\end{array}$ & $\begin{array}{l}\text { Conc. } \\
{[\mathbf{M}]}\end{array}$ & $\begin{array}{l}\text { Yield }^{\mathbf{b}} \\
{[\%]}\end{array}$ \\
\hline 1 & 1.0 & 1.1 & 3.1 & 1.1 & $1 \mathrm{~min}$ & 2 & 0.8 & 95 \\
$2^{\mathrm{c}}$ & 1.0 & 1.1 & 3.1 & 1.1 & $5 \mathrm{sec}$ & 2 & 0.8 & 96 \\
3 & 1.0 & 1.1 & 2.0 & 1.1 & $1 \mathrm{~min}$ & 2 & 0.8 & 97 \\
$4^{\mathrm{d}}$ & 1.0 & 1.1 & 1.0 & 1.1 & $1 \mathrm{~min}$ & 2 & 0.8 & 61 \\
$5^{\mathrm{d}}$ & 1.0 & 1.1 & - & 1.1 & $1 \mathrm{~min}$ & 2 & 0.8 & 31 \\
6 & 1.0 & 1.0 & 2.0 & 1.0 & $1 \mathrm{~min}$ & 2 & 0.8 & 95 \\
7 & 1.0 & 1.0 & 1.5 & 1.0 & $1 \mathrm{~min}$ & 2 & 0.8 & 76 \\
8 & 1.0 & 1.0 & 1.0 & 1.0 & $1 \mathrm{~min}$ & 2 & 0.8 & 58 \\
9 & 1.0 & 1.1 & 3.1 & 1.1 & $1 \mathrm{~min}$ & 0.5 & 0.8 & 95 \\
10 & 1.0 & 1.1 & 3.1 & 1.1 & $1 \mathrm{~min}$ & 0.1 & 0.8 & 99 \\
$11^{\mathrm{e}}$ & 1.0 & 1.1 & 3.1 & 1.1 & $1 \mathrm{~min}$ & 0.1 & 0.8 & 99 \\
12 & 1.0 & 1.1 & 2.0 & 1.1 & $1 \mathrm{~min}$ & 0.1 & 0.8 & 88 \\
13 & 1.0 & 1.1 & 3.1 & 1.1 & $1 \mathrm{~min}$ & 2 & 0.5 & 92 \\
14 & 1.0 & 1.1 & 3.1 & 1.1 & $1 \mathrm{~min}$ & 2 & 0.1 & 99 \\
\hline
\end{tabular}

Table S4. Optimization of reaction conditions for scale up experiment. ${ }^{a}$ Reactions were performed on $1 \mathrm{mmol}$ scale following the general procedure (III) for amide coupling reactions. ${ }^{\mathrm{b}}$ Isolated yields of the crude products (spectroscopically clean, no need for further purification).

${ }^{\mathrm{c}}$ The reaction was quenched immediately after the addition of COMU and submitted to work-up. ${ }^{\mathrm{d}}$ Incomplete conversion after $1 \mathrm{~min} .{ }^{\mathrm{e}}$ Reaction was performed on $3 \mathrm{~g}$ (15.3 mmol) scale. 


\section{Synthesis and Characterization of Amide Coupling Products}<smiles>COc1ccc(CCNC(=O)Cc2ccccc2)cc1OC</smiles>

\section{$N$-(3,4-dimethoxyphenethyl)-2-phenylacetamide}

(36): General procedure (III) was followed using phenyl acetic acid (1.1 mmol, $150 \mathrm{mg}, 1.1$ equiv.), $1.25 \mathrm{~mL}$ of a $2 \mathrm{wt} \% \mathrm{HPMC}$ in degassed Millipore water solution, 2,6-lutidine $(3.1 \mathrm{mmol}, 360 \mu \mathrm{L}$, 3.1 equiv.), 3,4-dimethoxyphenethylamine (1.0 mmol, $181 \mathrm{mg}, 1.0$ equiv.) and COMU (1.1 mmol, $471 \mathrm{mg}, 1.1$ equiv.). The reaction was quenched after $1 \mathrm{~min}$ by the addition of the sat. aq. sodium sulfate solution and submitted to work-up. The desired product was obtained in $80 \%$ yield $(239 \mathrm{mg}$ ) as a white solid. The spectral data are consistent with those reported in the literature (18).

${ }^{1} \mathbf{H}$ NMR $\left(500 \mathrm{MHz}, \mathrm{CDCl}_{3}\right) \delta$ 7.33-7.26 (m, 3H), 7.19-7.13 (m, 2H), $6.72(\mathrm{~d}, J=8.1 \mathrm{~Hz}$, $1 \mathrm{H}), 6.60(\mathrm{~d}, J=2.0 \mathrm{~Hz}, 1 \mathrm{H}), 6.55(\mathrm{dd}, J=8.1,2.0 \mathrm{~Hz}, 1 \mathrm{H}), 5.35(\mathrm{~s}, 1 \mathrm{H}, \mathrm{NH}), 3.86(\mathrm{~s}, 3 \mathrm{H})$, $3.82(\mathrm{~s}, 3 \mathrm{H}), 3.53(\mathrm{~s}, 2 \mathrm{H}), 3.45(\mathrm{td}, J=6.9,5.7 \mathrm{~Hz}, 2 \mathrm{H}), 2.68(\mathrm{t}, J=6.9 \mathrm{~Hz}, 2 \mathrm{H}) \mathrm{ppm}$.

${ }^{13}$ C NMR $\left(126 \mathrm{MHz}, \mathrm{CDCl}_{3}\right) \delta 170.8,149.0,147.6,134.8,131.2,129.4,128.9,127.3$, $120.6,111.8,111.3,55.9,55.8,43.9,40.7,35.0$ ppm.

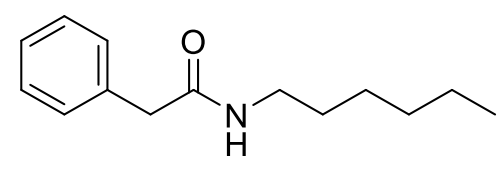

N-hexyl-2-phenylacetamide (37): General procedure (III) was followed using phenylacetic acid $(1.1 \mathrm{mmol}, 150 \mathrm{mg}$, 1.1 equiv.), $1.25 \mathrm{~mL}$ of a $2 \mathrm{wt} \%$ HPMC in degassed Millipore water solution, 2,6-lutidine (3.1 mmol, $360 \mu \mathrm{L}, 3.1$ equiv.), hexylamine (1.0 mmol, $132 \mu \mathrm{L}, 1.0$ equiv.), and COMU (1.1 mmol, $471 \mathrm{mg}, 1.1$ equiv.). The reaction was quenched after $1 \mathrm{~min}$ by the addition of the sat. aq. sodium sulfate solution and submitted to work-up. The desired product was obtained in $98 \%$ yield (214.2 mg) as a pale-yellow solid. The spectral data are consistent with those reported in the literature (18).

${ }^{1} \mathbf{H}$ NMR $\left(500 \mathrm{MHz}, \mathrm{CDCl}_{3}\right) \delta$ 7.35-7.28 (m, 2H), 7.28-7.21 (m, 3H), 5.67 (brs, 1H, NH), $3.52(\mathrm{~s}, 2 \mathrm{H}), 3.16(\mathrm{q}, J=6.7 \mathrm{~Hz}, 2 \mathrm{H}), 1.43-1.34(\mathrm{~m}, 2 \mathrm{H}), 1.29-1.15(\mathrm{~m}, 6 \mathrm{H}), 0.83(\mathrm{t}, J=$ $6.9 \mathrm{~Hz}, 3 \mathrm{H})$ ppm. 
${ }^{13} \mathbf{C}$ NMR $\left(126 \mathrm{MHz}, \mathrm{CDCl}_{3}\right) \delta 170.9,135.2,129.4,128.9,127.2,43.83,39.69,31.39$, $29.4,26.4,22.5,13.9$ ppm.

4-bromo- $N$-methoxy- $N$-methylbenzamide (39): General procedure (III) was
of a $2 \mathrm{wt} \% \mathrm{HPMC}$ in degassed Millipore water solution, 2,6-
lutidine $(3.1 \mathrm{mmol}, 360 \mu \mathrm{L}, 3.1 \quad$ equiv. $), \quad N, O$-dimethylhydroxyamine 1.1 equiv.). The reaction was quenched after $1 \mathrm{~min}$ by the addition of the sat. aq. $\mathrm{Na}_{2} \mathrm{SO}_{4}$ solution and submitted to work-up. Since the reaction was not complete after $1 \mathrm{~min}$ an additional purification had to be performed using column chromatography $(0-100 \%$ EtOAc in heptane). The desired product was obtained in $63 \%$ yield (155 mg). The spectral data are consistent with those reported in the literature (19).

${ }^{1}$ H NMR $\left(500 \mathrm{MHz}, \mathrm{CDCl}_{3}\right) \delta$ 7.62-7.46 (m, 4H), 3.52 (s, 3H), 3.34 (s, 3H) ppm.

${ }^{13}$ C NMR $\left(126 \mathrm{MHz}, \mathrm{CDCl}_{3}\right) \delta 168.9,132.8,131.3,130.1,125.3,61.2,33.6$ ppm.

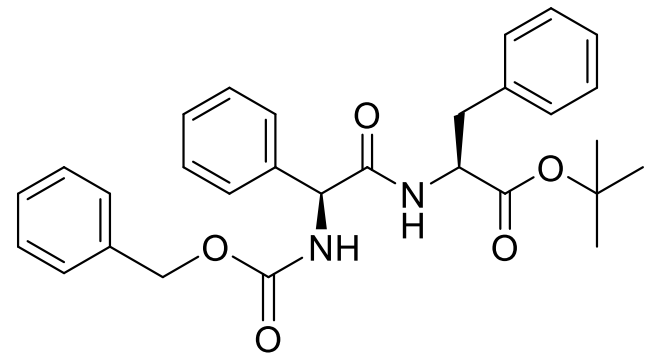

tert-Butyl (2-(((benzyloxy)carbonyl)amino)-2phenylacetyl)-L-phenylalaninate (40): General procedure (III) was followed using Z-PhG$\mathrm{OH}$ (1.1 mmol, $314 \mathrm{mg}, 1.1$ equiv.), $2.25 \mathrm{~mL}$ of a $2 \mathrm{wt} \%$ HPMC in degassed Millipore water solution, 2,6-lutidine $(3.1 \mathrm{mmol}, 360 \mu \mathrm{L}$, 3.1 equiv.), $\mathrm{H}-\mathrm{Phe}-\mathrm{O} t \mathrm{Bu}$ hydrochloride $(1.0 \mathrm{mmol}, 258 \mathrm{mg}, 1.0$ equiv.) and COMU (1.1 mmol, $471 \mathrm{mg}, 1.1$ equiv.). The reaction was quenched after $1 \mathrm{~min}$ by the addition of the sat. aq. Sodium sulfate solution and submitted to work-up. The desired product was obtained in $90 \%$ yield $(441.6 \mathrm{mg})$ as a pale yellow solid. The spectral data are consistent with those reported in the literature (1).

${ }^{1}$ H NMR $\left(500 \mathrm{MHz}, \mathrm{CDCl}_{3}\right) \delta 7.38-7.20(\mathrm{~m}, 13 \mathrm{H}), 7.10(\mathrm{~d}, J=7.0 \mathrm{~Hz}, 2 \mathrm{H}), 6.30(\mathrm{~d}$, $J=7.4 \mathrm{~Hz}, 1 \mathrm{H}), 6.10(\mathrm{~d}, J=6.6 \mathrm{~Hz}, 1 \mathrm{H}), 5.27-5.15(\mathrm{~m}, 1 \mathrm{H}), 5.12-5.00(\mathrm{~m}, 2 \mathrm{H}), 4.66(\mathrm{dt}$, $J=7.4,6.0 \mathrm{~Hz}, 1 \mathrm{H}), 3.12-3.00(\mathrm{~m}, 2 \mathrm{H}), 1.29$ (s, 9H) ppm. 
${ }^{13}$ C NMR (126 MHz, $\left.\mathrm{CDCl}_{3}\right)$ 169.7, 169.2, 155.7, 137.7, 136.3, 135.9, 129.5, 129.1, 128.5, $128.4,128.14,128.09,127.2,127.0,82.5,67.1,58.9,54.0,37.9,27.8$ ppm.

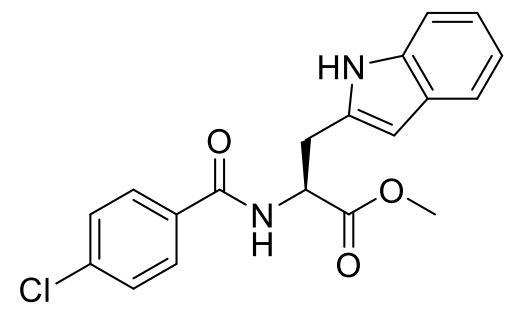

Methyl (4-chlorobenzoyl)-L-tryptophanate (41):

General procedure (III) was followed using 4chlorobenzoic acid (1.1 mmol, $172 \mathrm{mg}, 1.1$ equiv.), $1.25 \mathrm{~mL}$ of a $2 \mathrm{wt} \%$ HPMC in degassed Millipore water solution, 2,6-lutidine ( $3.1 \mathrm{mmol}, 360 \mu \mathrm{L}, 3.1$ equiv.), LTrp-OMe hydrochloride (1.0 mmol, $255 \mathrm{mg}, 1.0$ equiv.) and COMU (1.1 mmol, $471 \mathrm{mg}$, 1.1 equiv.). The reaction mixture was quenched after $1 \mathrm{~min}$ by the addition of sat. aq. $\mathrm{Na}_{2} \mathrm{SO}_{4}$ solution and submitted to work-up. Since the reaction was not complete after $1 \mathrm{~min}$ an additional purification had to be performed using column chromatography (0-100 \% EtOAc in heptane). The desired product was obtained in $66 \%$ yield $(236 \mathrm{mg})$ as a yellow solid. The spectral data are consistent with those reported in the literature (18).

${ }^{1} \mathbf{H}$ NMR $\left(500 \mathrm{MHz}, \mathrm{CDCl}_{3}\right) \delta 8.46-8.30(\mathrm{~m}, 1 \mathrm{H}), 7.61-7.56(\mathrm{~m}, 2 \mathrm{H}), 7.55-7.50(\mathrm{~m}, 1 \mathrm{H})$, 7.36-7.28 (m, 3H), 7.21-7.15 (m, 1H), 7.11-7.04 (m, 1H), 7.00-6.94 (m, 1H), 6.74$6.64(\mathrm{~m}, 1 \mathrm{H}), 5.12$ (dt, $J=7.7 \mathrm{~Hz}, 5.2 \mathrm{~Hz}, 1 \mathrm{H}), 3.73$ (s, 3H), 3.50-3.37 (m, 2H) ppm.

${ }^{13} \mathrm{C}$ NMR $\left(126 \mathrm{MHz}, \mathrm{CDCl}_{3}\right) \delta 172.4,166.2,138.0,136.3,132.1,128.8,128.6,127.6$, 123.0, 122.3, 119.7, 118.5, 111.6, 109.6, 53.8, 52.6, $27.6 \mathrm{ppm}$.

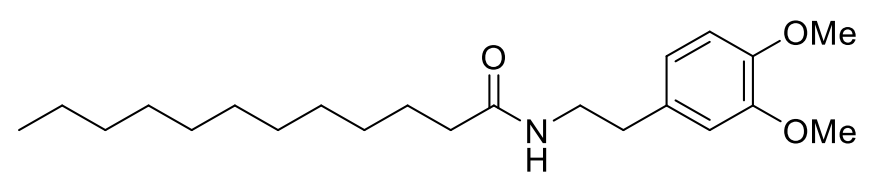

$N$-(3,4-dimethoxyphenethyl)dodecanamide (42): General procedure (III) was followed using lauric acid (1.1 mmol, $220 \mathrm{mg}, 1.1$ equiv.), $1.25 \mathrm{~mL}$ of a $2 \mathrm{wt} \% \mathrm{HPMC}$ in degassed Millipore water solution, 2,6-lutidine (3.1 mmol, $360 \mu \mathrm{L}, 3.1$ equiv.), 3,4dimethoxyphenethylamine (1.0 mmol, $181 \mathrm{mg}, 1.0$ equiv.), and COMU (1.1 mmol, $471 \mathrm{mg}, 1.1$ equiv.). The reaction was quenched after $1 \mathrm{~min}$ by the addition of the sat. aq. $\mathrm{Na}_{2} \mathrm{SO}_{4}$ solution and submitted to work-up. The desired product was obtained in $79 \%$ yield (288 $\mathrm{mg}$ ) as a yellow solid. The spectral data are consistent with those reported in the literature (18). 
${ }^{1} \mathbf{H}$ NMR $\left(500 \mathrm{MHz}, \mathrm{CDCl}_{3}\right) \delta$ 6.82-6.76 (m, 1H), 6.73-6.68 (m, 2H), $5.54(\mathrm{~s}, 1 \mathrm{H}, \mathrm{NH})$, $3.85(2 \mathrm{~s}, 6 \mathrm{H}), 3.48(\mathrm{q}, J=6.8 \mathrm{~Hz}, 2 \mathrm{H}), 2.74(\mathrm{t}, J=7.0 \mathrm{~Hz}, 2 \mathrm{H}), 2.11(\mathrm{t}, J=7.6 \mathrm{~Hz}, 2 \mathrm{H})$, 1.58 (h, $J=6.8 \mathrm{~Hz}, 2 \mathrm{H}), 1.29-1.22(\mathrm{~m}, 16 \mathrm{H}), 0.86$ (t, $J=6.8 \mathrm{~Hz}, 3 \mathrm{H}) \mathrm{ppm}$.

${ }^{13}$ C NMR (126 MHz, $\left.\mathrm{CDCl}_{3}\right) \delta 173.3,149.1,147.7,131.4,120.6,111.9,111.4,55.9,55.8$, 40.6, 36.9, 35.3, 31.9, 29.64, 29.62, 29.5, 29.4, 29.34, 29.31, 25.8, 22.7, 14.1 ppm.<smiles>O=C(Cc1ccccc1)N1CCN(c2cc(C(F)(F)F)cc(C(F)(F)F)c2)CC1</smiles>

\section{1-(4-(3,5-bis(trifluoromethyl)phenyl)piperazin-1-} yl)-2-phenylethan-1-one (43): General procedure (III) was followed using phenylacetic acid (1.1 mmol, $150 \mathrm{mg}, 1.1$ equiv.), $1.25 \mathrm{~mL}$ of a $2 \mathrm{wt} \%$ HPMC in degassed Millipore water solution, 2,6lutidine (3.1 mmol, $360 \mu \mathrm{L}, 3.1$ equiv.), 1-[3,5bis(trifluoromethyl)phenyl]piperazine (1.0 mmol, $298 \mathrm{mg}, 1.0$ equiv.) and COMU (1.1 mmol, $471 \mathrm{mg}, 1.1$ equiv.). The reaction was quenched after $1 \mathrm{~min}$ by the addition of the sat. aq. sodium sulfate solution and submitted to work-up. The desired product was obtained in quantitative yield $(416 \mathrm{mg})$ as a white solid.

${ }^{1} \mathbf{H}$ NMR $\left(500 \mathrm{MHz}, \mathrm{CDCl}_{3}\right) \delta$ 7.37-7.30 (m, 3H), 7.29-7.24 (m, 3H), $7.19(\mathrm{~s}, 2 \mathrm{H}), 3.83(\mathrm{t}$, $J=5.3 \mathrm{~Hz}, 2 \mathrm{H}), 3.80(\mathrm{~s}, 2 \mathrm{H}), 3.63(\mathrm{t}, J=5.2 \mathrm{~Hz}, 2 \mathrm{H}), 3.26(\mathrm{t}, J=5.3 \mathrm{~Hz}, 2 \mathrm{H}), 3.08$ (t, $J=$ $5.2 \mathrm{~Hz}, 2 \mathrm{H}) \mathrm{ppm}$.

${ }^{13}$ C NMR (126 MHz, $\left.\mathrm{CDCl}_{3}\right) \delta 169.6,151.2,134.7,132.6(\mathrm{q}, J=33.2 \mathrm{~Hz}), 128.9,128.5$, $127.1,124.5,122.3,120.2,115.1,112.7,48.3,48.1,45.5,41.3,41.1 \mathrm{ppm}$.

${ }^{19} \mathbf{F}$ NMR $\left(471 \mathrm{MHz}, \mathrm{CDCl}_{3}\right) \delta-61.07(\mathrm{~s}, 6 \mathrm{~F}) \mathrm{ppm}$.

HRMS (ESI) $\mathrm{m} / \mathrm{z}$ calcd. for $\mathrm{C}_{20} \mathrm{H}_{19} \mathrm{~F}_{6} \mathrm{~N}_{2} \mathrm{O}\left([\mathrm{M}+\mathrm{H}]^{+}\right)$417.1402, found 417.1398.

IR (film) vmax 1645, 1618, 1404, 1271, 1236, 1219, 1120, 1106, 992, 963, 849, 736, 693, $680 \mathrm{~cm}^{-1}$.

Melting point $155.5^{\circ} \mathrm{C}$ 
$N$-hexyl-3-phenylpropiolamide (44): General procedure

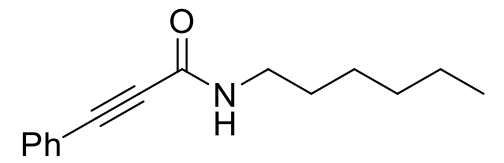

(III) was followed using phenylpropiolic acid $(1.1 \mathrm{mmol}$,

Millipore water solution, 2,6-lutidine ( $3.1 \mathrm{mmol}, 360 \mu \mathrm{L}, 3.1$ equiv.), hexylamine (1.0 mmol, $132 \mu \mathrm{L}, 1.0$ equiv.) and COMU (1.1 mmol, $471 \mathrm{mg}, 1.1$ equiv.). The reaction was quenched after $1 \mathrm{~min}$ by the addition of the sat. aq. sodium sulfate solution and submitted to work-up. The desired product was obtained in $70 \%$ yield $(160.5 \mathrm{mg})$ as a colorless oil. The spectral data are consistent with those reported in the literature (18).

An aliquot was further purified by preparative SFC for obtaining ${ }^{1} \mathrm{H}$ - and ${ }^{13} \mathrm{C}-\mathrm{NMR}$ spectra. For comparison ${ }^{1} \mathrm{H}$-NMR spectra of the crude and the purified sample are shown in Fig. S13.

${ }^{1} \mathbf{H}$ NMR $\left(500 \mathrm{MHz}, \mathrm{CDCl}_{3}\right) \delta$ 7.57-7.49 (m, 2H), 7.45-7.32 (m, 3H), $6.05(\mathrm{~s}, 1 \mathrm{H}, \mathrm{NH})$, $3.34(\mathrm{td}, J=7.2,5.9 \mathrm{~Hz}, 2 \mathrm{H}), 1.64-1.50(\mathrm{~m}, 2 \mathrm{H}), 1.39-1.23(\mathrm{~m}, 6 \mathrm{H}), 0.96-0.80(\mathrm{~m}$, 3H) $\mathrm{ppm}$.

${ }^{13} \mathrm{C}$ NMR $\left(126 \mathrm{MHz}, \mathrm{CDCl}_{3}\right) \delta 153.4,132.5,129.9,128.5,120.3,84.4,83.2,40.0,31.4$, 29.3, 26.6, 22.5, $14.0 \mathrm{ppm}$. 


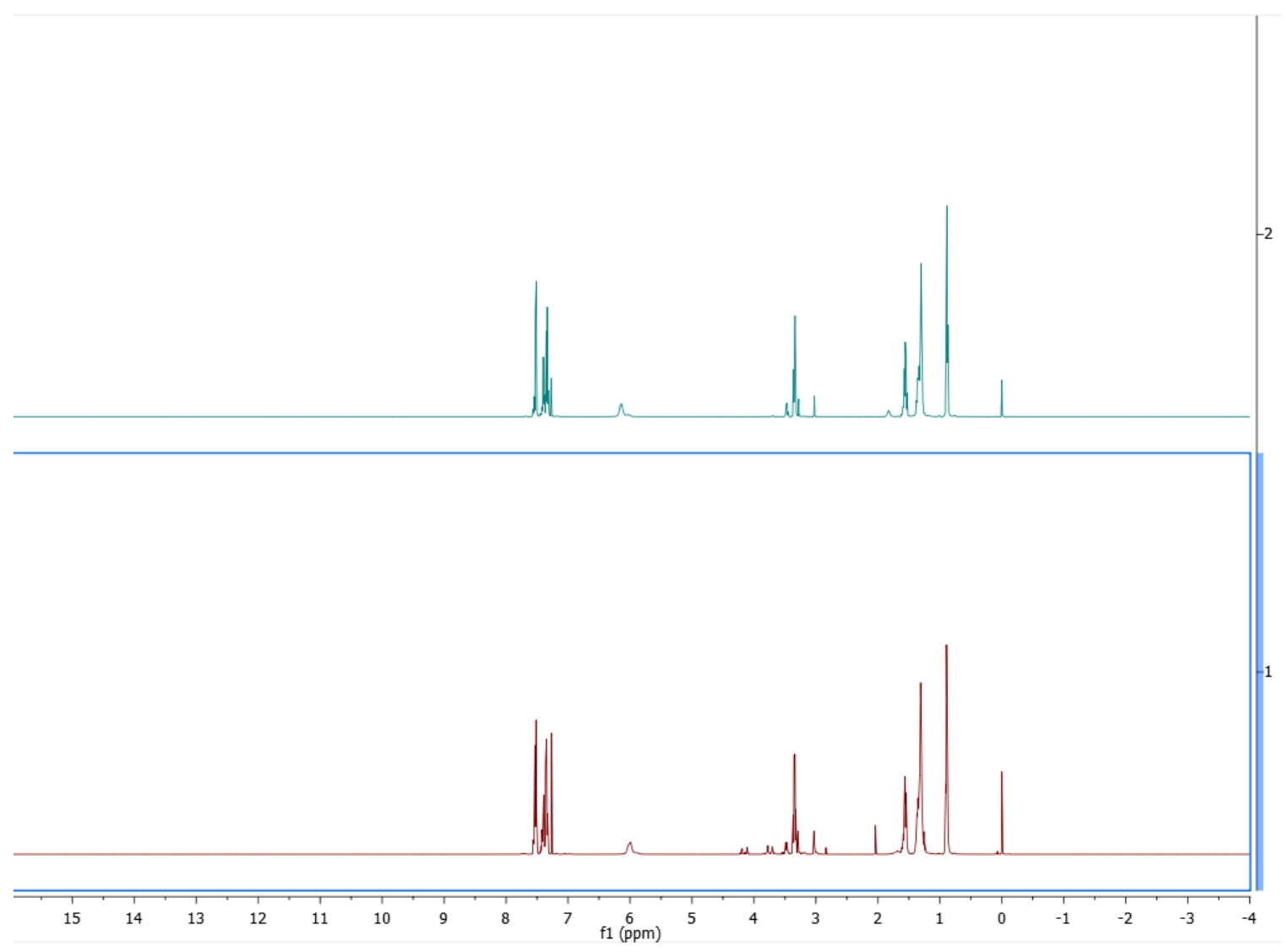

Figure S13. Comparison of ${ }^{1} \mathrm{H}-\mathrm{NMR}$ spectra of the crude (bottom red spectrum) and a purified aliquot (upper green spectrum).

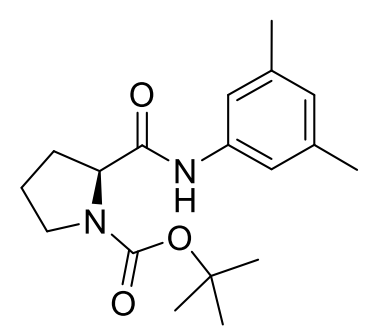

(S)-tert-butyl 2-((3,5-dimethylphenyl)carbamoyl)pyrrolidine-1carboxylate (45): General procedure (III) was followed using BocPro-OH (0.55 mmol, $118 \mathrm{mg}, 1.1$ equiv.), $625 \mu \mathrm{L}$ of a $2 \mathrm{wt} \%$ HPMC in degassed Millipore water solution, 2,6lutidine (1.55 mmol, $\quad 181 \mu \mathrm{L}, \quad 3.1 \quad$ equiv.), 3,5dimethylaniline ( $0.5 \mathrm{mmol}, 62 \mu \mathrm{L}, 1.0$ equiv.) and $\mathrm{COMU}(0.55 \mathrm{mmol}, 236 \mathrm{mg}$, 1.1 equiv.). The reaction mixture was quenched after $1 \mathrm{~min}$ by the addition of sat. aq. $\mathrm{Na}_{2} \mathrm{SO}_{4}$ solution and submitted to work-up. The desired product was obtained in $78 \%$ yield $(128 \mathrm{mg})$ as a white solid. Due to hinderance of the rotation around the carbamate bond two rotamers were obtained which lead to a doubled set of signals in the NMR spectra. 
${ }^{1}$ H NMR (500 MHz, DMSO-d $\left.d_{6}\right) \delta 9.79(\mathrm{~s}, 1 \mathrm{H}), 7.22(\mathrm{~s}, 2 \mathrm{H}), 6.68(\mathrm{~s}, 1 \mathrm{H}), 4.28-4.12(\mathrm{~m}$, $1 \mathrm{H}), 3.45-3.37(\mathrm{~m}, 1 \mathrm{H}), 3.32-3.26(\mathrm{~m}, 1 \mathrm{H}), 2.22(\mathrm{~s}, 6 \mathrm{H}), 2.20-2.07(\mathrm{~m}, 1 \mathrm{H}), 1.97-$ $1.71(\mathrm{~m}, 3 \mathrm{H}), 1.39(\mathrm{~s}, 3 \mathrm{H}), 1.27(\mathrm{~s}, 6 \mathrm{H}) \mathrm{ppm}$.

${ }^{13}$ C NMR $\left(126\right.$ MHz, DMSO- $\left.d_{6}\right) \delta 171.4,170.9,153.6,153.2,139.0,137.6,124.7,124.6$, $117.0,116.9,78.6,78.4,60.3,60.0,46.7,46.5,31.0,30.2,28.2,28.0,23.9,23.3,21.1$ ppm.

HRMS (ESI) m/z calcd. for $\mathrm{C}_{18} \mathrm{H}_{27} \mathrm{~N}_{2} \mathrm{O}_{3}\left([\mathrm{M}+\mathrm{H}]^{+}\right)$319.2016, found 319.2014.

IR (film) vmax 1694, 1670, 1617, 1560, 1365, 1161, 1126, 845, 690, $532 \mathrm{~cm}^{-1}$.

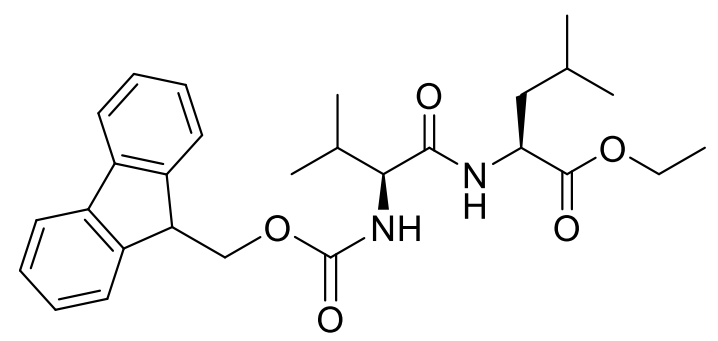

Ethyl (((9H-fluoren-9-yl)methoxy)carbonyl)valyl-L-leucinate (46): General procedure (III) was followed using FmocVal-OH (1.1 mmol, $373 \mathrm{mg}, 1.1$ equiv.), 1.25 $\mathrm{mL}$ of a $2 \mathrm{wt} \%$ HPMC in degassed Millipore water solution, 2,6-lutidine (3.1 mmol, $360 \mu \mathrm{L}, 3.1$ equiv.), L-leucine ethyl ester hydrochloride (1.0 mmol, $196 \mathrm{mg}, 1.0$ equiv.) and COMU (1.1 mmol, $471 \mathrm{mg}, 1.1$ equiv.) The reaction was quenched after $1 \mathrm{~min}$ by the addition of the sat. aq. sodium sulfate solution and submitted to work-up. The desired product was obtained in $90 \%$ yield (431.2 mg) as a white solid. The spectral data are consistent with those reported in the literature (18).

${ }^{1}$ H NMR $\left(500 \mathrm{MHz}, \mathrm{DMSO}-d_{6}\right) \delta 8.24(\mathrm{~d}, J=7.4 \mathrm{~Hz}, 1 \mathrm{H}), 7.89(\mathrm{~d}, J=7.4 \mathrm{~Hz}, 2 \mathrm{H}), 7.75$ (t, $J=6.7 \mathrm{~Hz}, 2 \mathrm{H}), 7.45-7.36(\mathrm{~m}, 3 \mathrm{H}), 7.34-7.29$ (m, 2H), 4.33-4.19 (m, 4H), 4.12-4.00 (m, 2H), $3.92(\mathrm{dd}, J=9.1,7.4 \mathrm{~Hz}, 1 \mathrm{H}), 1.99(\mathrm{~h}, J=6.8 \mathrm{~Hz}, 1 \mathrm{H}), 1.71-1.45(\mathrm{~m}, 3 \mathrm{H}), 1.16(\mathrm{t}$, $J=7.1 \mathrm{~Hz}, 3 \mathrm{H}), 0.93-0.86(\mathrm{~m}, 9 \mathrm{H}), 0.83(\mathrm{~d}, J=6.5 \mathrm{~Hz}, 3 \mathrm{H}) \mathrm{ppm}$.

${ }^{13}$ C NMR (126 MHz, DMSO-d $) \delta$ 172.7, 171.8, 156.5, 144.4, 144.2, 141.2, 128.1, 127.5, $125.85,125.82,120.5,66.1,60.84,60.3,50.8,47.1,30.9,24.6,23.2,21.7,19.6,18.7,14.5$ ppm. 


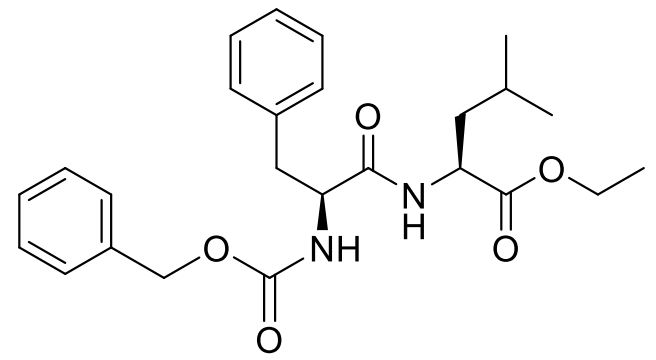

Ethyl ((benzyloxy)carbonyl)phenylalanyl-Lleucinate (47): General procedure (III) was followed using Z-Phe-OH (1.1 mmol, $329 \mathrm{mg}$, 1.1 equiv.), $1.25 \mathrm{~mL}$ of a $2 \mathrm{wt} \% \mathrm{HPMC}$ in degassed Millipore water solution, 2,6lutidine ( $3.1 \mathrm{mmol}, 360 \mu \mathrm{L}, 3.1$ equiv.), L-leucine ethyl ester hydrochloride (1.0 mmol, $196 \mathrm{mg}, 1.0$ equiv.) and COMU (1.1 mmol, $471 \mathrm{mg}$, 1.1 equiv.). The reaction was quenched after $1 \mathrm{~min}$ by the addition of the sat. aq. sodium sulfate solution and submitted to work-up. The desired product was obtained in $95 \%$ yield $(420.2 \mathrm{mg})$ as a white solid. The spectral data are consistent with those reported in the literature (18).

${ }^{1}$ H NMR $\left(500 \mathrm{MHz}, \mathrm{DMSO}-d_{6}\right) \delta 8.37(\mathrm{~d}, J=7.7 \mathrm{~Hz}, 1 \mathrm{H}), 7.47(\mathrm{~d}, J=8.8 \mathrm{~Hz}, 1 \mathrm{H}), 7.37-$ 7.15 (m, 10H), 4.94 (s, 2H), 4.36-4.22 (m, 2H), 4.15-4.00 (m, 2H), 3.01 (dd, $J=13.9,3.8$ $\mathrm{Hz}, 1 \mathrm{H}), 2.74$ (dd, $J=13.9,10.8 \mathrm{~Hz}, 1 \mathrm{H}), 1.72-1.46(\mathrm{~m}, 3 \mathrm{H}), 1.17$ (t, $J=7.1 \mathrm{~Hz}, 3 \mathrm{H}), 0.92$ (d, $J=6.5 \mathrm{~Hz}, 3 \mathrm{H}), 0.86(\mathrm{~d}, J=6.5 \mathrm{~Hz}, 3 \mathrm{H}) \mathrm{ppm}$.

${ }^{13}$ C NMR (126 MHz, DMSO- $\left.d_{6}\right) \delta 172.8,172.3,156.3,138.5,137.5,129.7,128.7,128.5$, $128.1,127.9,126.7,65.6,60.9,56.3,50.9,37.8,24.7,23.2,21.8,14.5$ ppm.

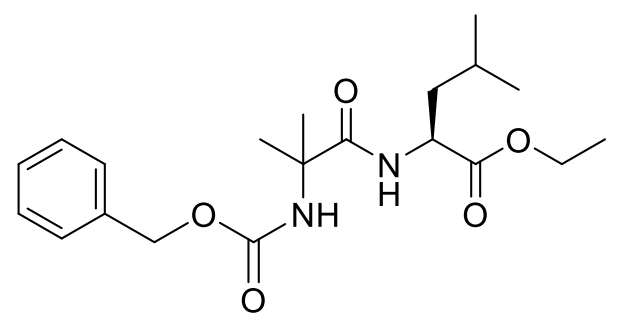

Ethyl (2-(((benzyloxy)carbonyl)amino)-2methylpropanoyl)-L-leucinate (48): General procedure (III) was followed using Z-Aib$\mathrm{OH}$ (1.1 mmol, $261 \mathrm{mg}, 1.1$ equiv.), $1.25 \mathrm{~mL}$ of a $2 \mathrm{wt} \%$ HPMC in degassed Millipore water solution, 2,6-lutidine (3.1 mmol, $360 \mu \mathrm{L}, 3.1$ equiv.), L-leucine ethyl ester hydrochloride (1.0 mmol, $196 \mathrm{mg}, 1.0$ equiv.) and COMU (1.1 mmol, $471 \mathrm{mg}, 1.1$ equiv.). The reaction was quenched after $1 \mathrm{~min}$ by the addition of the sat. aq. sodium sulfate solution and submitted to work-up. The desired product was obtained in $95 \%$ yield $(358.2 \mathrm{mg})$ as a pale-yellow solid. The spectral data are consistent with those reported in the literature (18). 
${ }^{1}$ H NMR (500 MHz, DMSO-d $\left.d_{6}\right) 7.73(\mathrm{~d}, J=8.0 \mathrm{~Hz}, 1 \mathrm{H}), 7.41-7.27(\mathrm{~m}, 5 \mathrm{H}), 7.22(\mathrm{~s}$, $1 \mathrm{H}), 4.99(\mathrm{q}, J=12.7 \mathrm{~Hz}, 2 \mathrm{H}), 4.27-4.19(\mathrm{~m}, 1 \mathrm{H}), 4.12-4.00(\mathrm{~m}, 2 \mathrm{H}), 1.67-1.55(\mathrm{~m}, 2 \mathrm{H})$, $1.49-1.40(\mathrm{~m}, 1 \mathrm{H}), 1.35(\mathrm{~s}, 6 \mathrm{H}), 1.16(\mathrm{t}, J=7.1 \mathrm{~Hz}, 3 \mathrm{H}), 0.85(\mathrm{~d}, J=6.2 \mathrm{~Hz}, 3 \mathrm{H}), 0.81$ (d, $J=6.2 \mathrm{~Hz}, 3 \mathrm{H}) \mathrm{ppm}$.

${ }^{13}$ C NMR (126 MHz, DMSO- $\left.d_{6}\right) \delta 174.6,172.9,155.0,137.5,128.7,128.2,128.1,65.5$, $60.7,56.3,50.9,39.9,25.8,25.0,24.6,23.3,21.6,14.5 \mathrm{ppm}$.

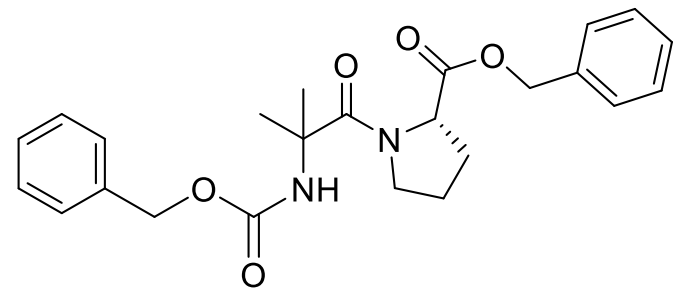

Benzyl (2-(((benzyloxy)carbonyl)amino)-2methylpropanoyl)-L-prolinate (49):

General procedure (III) was followed using ZAib-OH (1.1 mmol, $261 \mathrm{mg}, 1.1$ equiv.), $1.25 \mathrm{~mL}$ of a $2 \mathrm{wt} \%$ HPMC in degassed Millipore water solution, 2,6-lutidine ( $3.1 \mathrm{mmol}, 360 \mu \mathrm{L}, 3.1$ equiv.), L-proline benzyl ester hydrochloride (1.0 mmol, $242 \mathrm{mg}, 1.0$ equiv.) and COMU (1.1 mmol, $471 \mathrm{mg}, 1.1$ equiv.) The reaction was quenched after $1 \mathrm{~min}$ by the addition of the sat. aq. sodium sulfate solution and submitted to work-up. The desired product was obtained in $82 \%$ yield $(337.7 \mathrm{mg})$ as a pale-yellow solid. The spectral data are consistent with those reported in the literature (18).

An aliquot was further purified by preparative SFC for obtaining ${ }^{1} \mathrm{H}$ - and ${ }^{13} \mathrm{C}-\mathrm{NMR}$ spectra. For comparison ${ }^{1} \mathrm{H}$-NMR spectra of the crude and the purified sample are shown in Fig. S14.

${ }^{1} \mathbf{H}$ NMR $\left(500 \mathrm{MHz}\right.$, DMSO- $\left.d_{6}\right) \delta 7.75(\mathrm{~s}, 1 \mathrm{H}), 7.40-7.23(\mathrm{~m}, 10 \mathrm{H}), 5.13-4.93(\mathrm{~m}, 4 \mathrm{H})$, 4.31 (dd, $J=9.1,3.1 \mathrm{~Hz}, 1 \mathrm{H}), 3.56-3.58(\mathrm{~m}, 1 \mathrm{H}), 3.32-3.23$ (m, 1H), 2.00-1.89 (m, 1H), $1.87-1.66(\mathrm{~m}, 3 \mathrm{H}), 1.35(\mathrm{~s}, 3 \mathrm{H}), 1.24(\mathrm{~s}, 3 \mathrm{H}) \mathrm{ppm}$.

${ }^{13}$ C NMR (126 MHz, DMSO- $\left.d_{6}\right) \delta 172.4,172.1,154.7,137.5,136.6,128.8,128.7,128.5$, $128.4,128.4,128.2,66.1,65.7,60.7,56.2,47.7,27.8,26.1,25.8,24.8 \mathrm{ppm}$. 


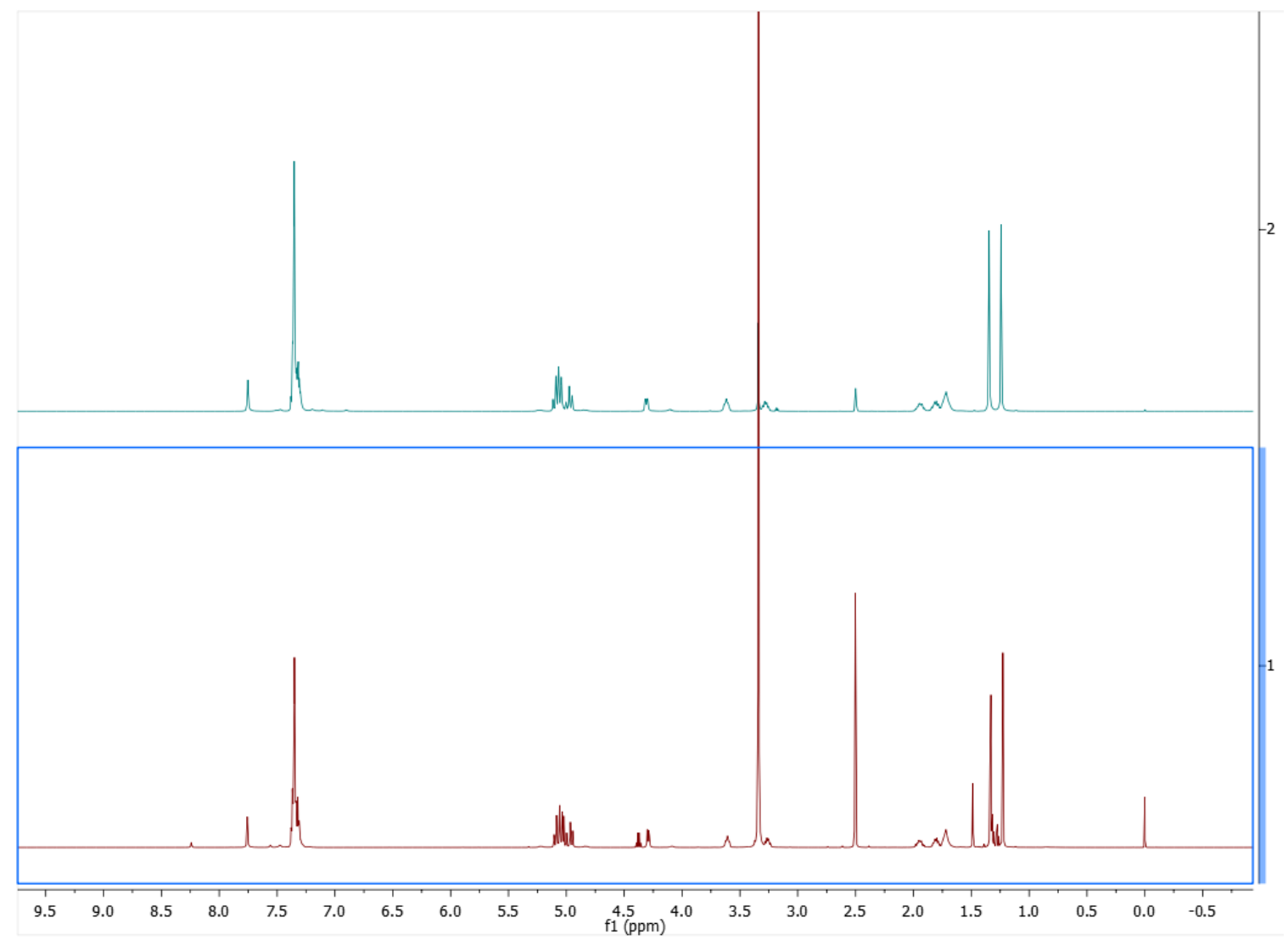

Figure S14. Comparison of ${ }^{1} \mathrm{H}-\mathrm{NMR}$ spectra of the crude (bottom red spectrum) and a purified aliquot (top green spectrum).<smiles>O=C(N[C@@H](Cc1ccccc1)C(=O)N1CCC[C@H]1C(=O)OCc1ccccc1)OCc1ccccc1</smiles>

Benzyl ((benzyloxy)carbonyl)phenylalanyl-Lprolinate (50):

General procedure (III) was followed using ZPhe-OH (1.1 mmol, $329 \mathrm{mg}, 1.1$ equiv.), $1.25 \mathrm{~mL}$ of a $2 \mathrm{wt} \%$ HPMC in degassed Millipore water solution, 2,6-lutidine ( $3.1 \mathrm{mmol}, 360 \mu \mathrm{L}, 3.1$ equiv.), L-proline benzyl ester hydrochloride (1.0 mmol, $242 \mathrm{mg}, 1.0$ equiv.) and COMU (1.1 mmol, $471 \mathrm{mg}, 1.1$ equiv.). The reaction was quenched after $1 \mathrm{~min}$ by the addition of the sat. aq. sodium sulfate solution and submitted to work-up. The desired product was obtained in $92 \%$ yield (445.7 $\mathrm{mg}$ ) as a pale-yellow oil. The spectral data are consistent with those reported in the literature (18). 
An aliquot was further purified by preparative SFC for obtaining ${ }^{1} \mathrm{H}$ - and ${ }^{13} \mathrm{C}-\mathrm{NMR}$ spectra. For comparison ${ }^{1} \mathrm{H}$-NMR spectra of the crude and the purified sample are shown in Fig. S15.

${ }^{1}$ H NMR (500 MHz, DMSO-d $d_{6} \delta 7.72(\mathrm{~d}, J=8.4 \mathrm{~Hz}, 1 \mathrm{H}), 7.41-7.17(\mathrm{~m}, 15 \mathrm{H}), 5.14(\mathrm{~s}$, 2H), 4.95 (s, 2H), 4.49-4.40 (m, 2H), 3.76-3.68 (m, 1H), 3.59-3.51 (m, 1H), 2.88 (dd, $J=14.1,4.2 \mathrm{~Hz}, 1 \mathrm{H}), 2.76(\mathrm{dd}, J=14.1,10.0 \mathrm{~Hz}, 1 \mathrm{H}), 2.24-2.14$ (m, 1H), 1.97-1.81 (m, 3H) ppm.

${ }^{13}$ C NMR (126 MHz, DMSO- $\left.d_{6}\right) \delta$ 172.0, 170.7, 156.38, 138.2, 137.4, 136.4, 129.7, 128.9, $128.8,128.6,128.5,128.24,128.20,128.0,127.9,126.8,66.3,65.8,59.2,54.7,46.9,36.8$, 29.0, $25.2 \mathrm{ppm}$.

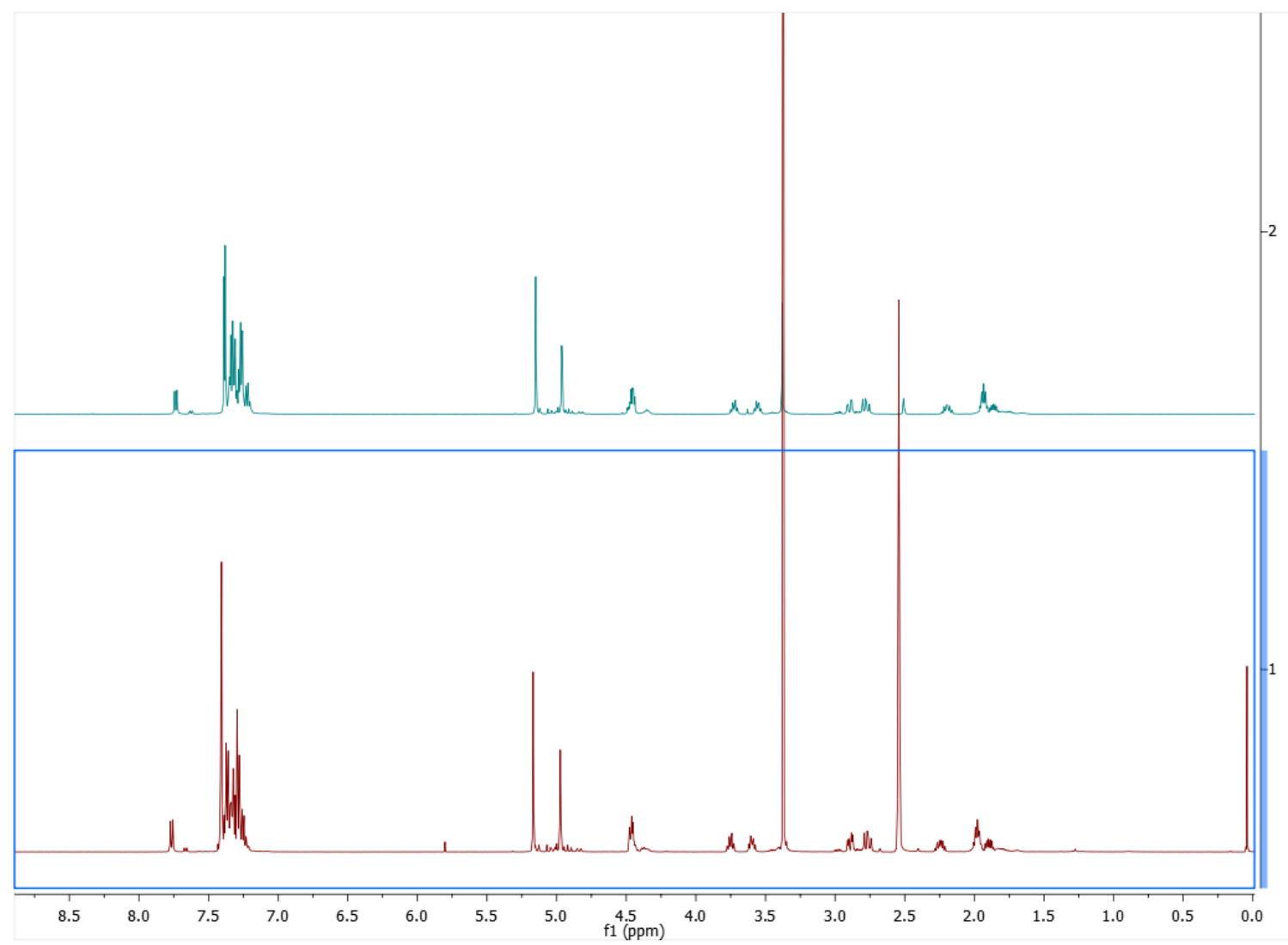

Figure S15. Comparison of ${ }^{1} \mathrm{H}-\mathrm{NMR}$ spectra of the crude (bottom red spectrum) and a purified aliquot (top green spectrum). 


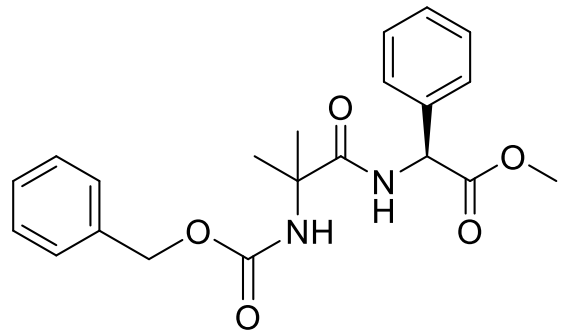

\section{Methyl (S)-2-(2-(((benzyloxy)carbonyl)amino)-2-} methylpropanamido)-2-phenylacetate

(51a):

General procedure (III) was followed using Z-Aib$\mathrm{OH}(1.1 \mathrm{mmol}, 261 \mathrm{mg}, 1.1$ equiv.), $1.25 \mathrm{~mL}$ of a $2 \mathrm{wt} \%$ HPMC in degassed Millipore water solution, 2,6-lutidine (3.1 mmol, $360 \mu \mathrm{L}, 3.1$ equiv.), L-phenylglycine methyl ester hydrochloride (1.0 mmol, $202 \mathrm{mg}, 1.0$ equiv.) and COMU (1.1 mmol, $471 \mathrm{mg}, 1.1$ equiv.). The reaction was quenched after $1 \mathrm{~min}$ by the addition of the sat. aq. sodium sulfate solution and submitted to work-up. The desired product was obtained in $96 \%$ yield (369.7 mg) as a yellow oil. The spectral data are consistent with those reported in the literature (18).

${ }^{1}$ H NMR (500 MHz, DMSO-d6) $\delta 8.13(\mathrm{~d}, J=7.3 \mathrm{~Hz}, 1 \mathrm{H}), 7.37-7.30$ (m, 11H), 5.40 (d, $J=7.2 \mathrm{~Hz}, 1 \mathrm{H}), 5.05-4.95(\mathrm{~m}, 2 \mathrm{H}), 3.63(\mathrm{~s}, 3 \mathrm{H}), 1.38$ (s, 3H), 1.37 (s, 3H) ppm.

${ }^{13}$ C NMR (126 MHz, DMSO-d6) 174.6, 171.4, 155.3, 137.4, 137.1, 128.9, 128.8, 128.5, $128.2,128.1,128.0,65.6,56.8,56.5,52.7,25.6,25.2 \mathrm{ppm}$.

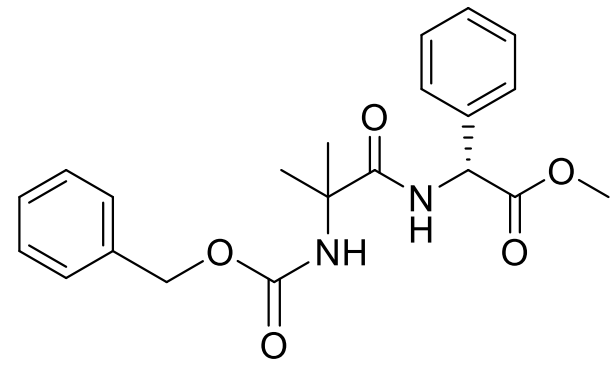

\section{Methyl (R)-2-(2-(((benzyloxy)carbonyl)amino)-} 2-methylpropanamido)-2-phenylacetate (51b):

General procedure (III) was followed using Z-Aib$\mathrm{OH}(0.55 \mathrm{mmol}, 130 \mathrm{mg}, 1.1$ equiv. $), 625 \mu \mathrm{L}$ of a $2 \mathrm{wt} \%$ HPMC in degassed Millipore water solution, 2,6-lutidine (1.55 mmol, $181 \mu \mathrm{L}, 3.1$ equiv.), Dphenylglycine methyl ester hydrochloride $(0.5 \mathrm{mmol}, 108 \mathrm{mg}, 1.0$ equiv.) and COMU ( $0.55 \mathrm{mmol}, 236 \mathrm{mg}, 1.1$ equiv.). The reaction was quenched after $1 \mathrm{~min}$ by the addition of the sat. aq. sodium sulfate solution and submitted to the work-up procedure described in section 9.1. The desired product was obtained in $76 \%$ yield $(146 \mathrm{mg})$ as a yellow oil. The spectral data are consistent with those reported in the literature (18).

${ }^{1}$ H NMR (500 MHz, DMSO-d $\left.)\right) \delta 8.15(\mathrm{~d}, J=7.1 \mathrm{~Hz}, 1 \mathrm{H}), 7.42-7.24(\mathrm{~m}, 11 \mathrm{H}), 5.40(\mathrm{~d}$, $J=7.4 \mathrm{~Hz}, 1 \mathrm{H}), 5.06-4.93$ (m, 2H), 3.63 (s, 3H), 1.38 (s, 3H), 1.37 (s, 3H) ppm.

${ }^{13}$ C NMR (126 MHz, DMSO-d6) 174.1, 170.9, 154.8, 137.0, 136.6, 128.5, 128.3, 128.0, $127.7,127.6,127.5,65.2,56.3,56.0,52.3,25.2,24.8 \mathrm{ppm}$. 


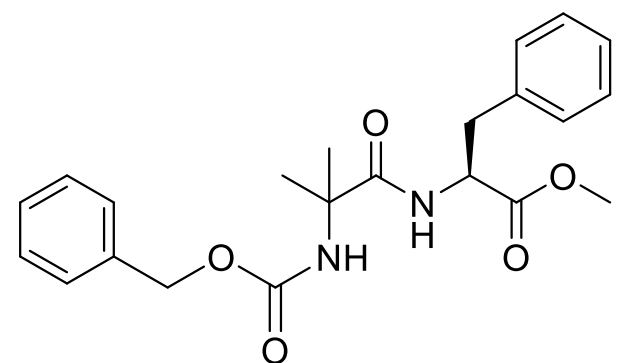

Methyl (S)-2-(2-(((benzyloxy)carbonyl)amino)2-methylpropanamido)-2-phenylpropanoate (52a): General procedure (III) was followed using Z-Aib-OH (0.55 mmol, $130 \mathrm{mg}, 1.1$ equiv. $), 625 \mu \mathrm{L}$ of a $2 \mathrm{wt} \% \mathrm{HPMC}$ in degassed Millipore water solution, 2,6-lutidine (1.55 mmol, $181 \mu \mathrm{L}, 3.1$ equiv. $)$ L-Phe-OMe hydrochloride $(0.5 \mathrm{mmol}, 108 \mathrm{mg}, 1.0$ equiv. $)$ and COMU ( $0.55 \mathrm{mmol}, 236 \mathrm{mg}, 1.1$ equiv.). The reaction was quenched after $1 \mathrm{~min}$ by the addition of the sat. aq. $\mathrm{Na}_{2} \mathrm{SO}_{4}$ solution and submitted to work-up. The desired product was obtained in $86 \%$ yield $(172 \mathrm{mg})$ as a yellow solid. The spectral data are consistent with those reported in the literature (18).

${ }^{1}$ H NMR $\left(500 \mathrm{MHz}, \mathrm{DMSO}-d_{6}\right) \delta 7.76(\mathrm{~d}, J=6.7 \mathrm{~Hz}, 1 \mathrm{H}), 7.45-7.10(\mathrm{~m}, 11 \mathrm{H}), 5.08-$ $4.87(\mathrm{~m}, 2 \mathrm{H}), 4.47(\mathrm{td}, J=8.3 \mathrm{~Hz}, 5.6 \mathrm{~Hz}, 1 \mathrm{H}), 3.60(\mathrm{~s}, 3 \mathrm{H}), 3.06-2.92(\mathrm{~m}, 2 \mathrm{H}), 1.27$ (s, 6H) ppm.

${ }^{13}$ C NMR (126 MHz, DMSO- $\left.d_{6}\right)$ 174.1, 171.9, 154.6, 137.3, 137.0, 129.2, 128.3, 128.1, $127.7,127.6,126.5,65.1,55.9,53.5,51.8,36.5,25.1,24.8$ ppm.

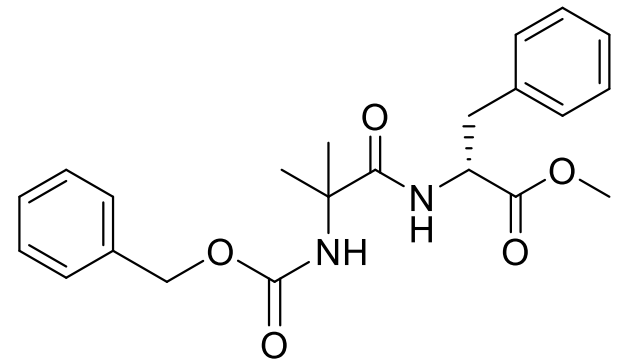

Methyl (R)-2-(2-(((benzyloxy)carbonyl)amino)2-methylpropanamido)-2-phenylpropanoate (52b): General procedure (III) was followed using Z-Aib-OH (0.55 mmol, $130 \mathrm{mg}, 1.1$ equiv.), $625 \mu \mathrm{L}$ of a $2 \mathrm{wt} \% \mathrm{HPMC}$ in degassed Millipore water solution, 2,6-lutidine (1.55 mmol, $181 \mu \mathrm{L}, 3.1$

equiv.), D-Phe-OMe hydrochloride (0.5 mmol, $108 \mathrm{mg}, 1.0$ equiv.) and COMU (0.55 mmol, $236 \mathrm{mg}, 1.1$ equiv.). The reaction was quenched after $1 \mathrm{~min}$ by the addition of the sat. aq. $\mathrm{Na}_{2} \mathrm{SO}_{4}$ solution and submitted to the work-up procedure described in section 9.1. The desired product was obtained in $81 \%$ yield $(161 \mathrm{mg})$ as a white solid. The spectral data are consistent with those reported in the literature (18). 
${ }^{1}$ H NMR (500 MHz, DMSO- $\left.d_{6}\right) \delta$ 7.95-7.66 (m 1H), 7.53-7.15 (m, $\left.11 \mathrm{H}\right), 5.14-4.84(\mathrm{~m}$, 2H), 4.47 (td, $J=8.3 \mathrm{~Hz}, 5.4 \mathrm{~Hz}, 1 \mathrm{H}), 3.60$ (s, 3H), 3.09-2.91 (m, 2H), 1.27 (s, 6H) ppm. ${ }^{13}$ C NMR (126 MHz, DMSO- $\left.d_{6}\right)$ 174.1, 171.9, 154.6, 137.3, 137.0, 129.2, 128.3, 128.1, $127.7,127.7,126.5,65.1,55.9,53.5,51.8,36.5,25.1,24.8 \mathrm{ppm}$. 


\section{Control Reactions in Water for Amide Couplings}

In order to validate whether HPMC has a beneficial impact on different amide couplings, control reactions using water as the reaction solvent were conducted. In this course, four different amide couplings were investigated with different substrates (see Fig. S16). For reaction $\mathrm{A}$ and $\mathrm{D}$, a positive impact of HPMC on the yield and the conversion was observed. For the reactions to the dipeptides 47 and 52 HPMC showed no impact on the reaction yield.

A

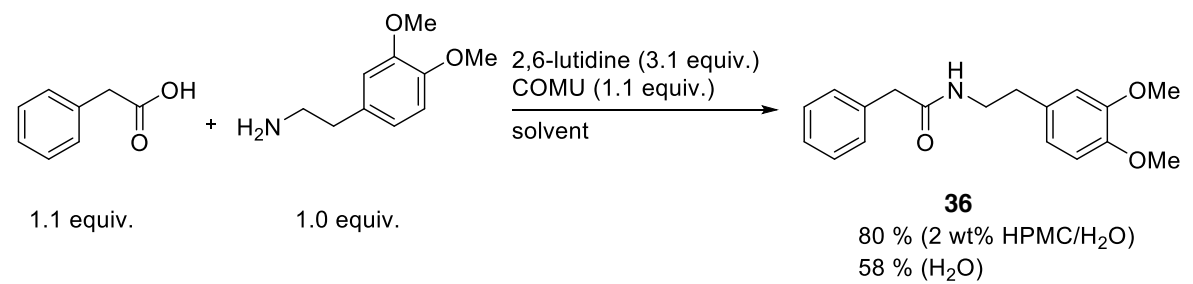

B

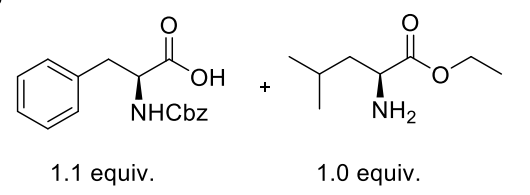

2,6-lutidine (3.1 equiv.) solvent<smiles>CCOC(=O)C(CC(C)C)NC(=O)[C@H](N)Cc1ccccc1</smiles>

$95 \%\left(2 \mathrm{wt} \% \mathrm{HPMC} / \mathrm{H}_{2} \mathrm{O}\right)$ $95 \%\left(\mathrm{H}_{2} \mathrm{O}\right)$

C

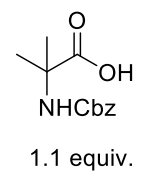

1.1 equiv.<smiles>COC(=O)[C@H](N)Cc1ccccc1</smiles>

1.0 equiv.

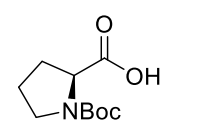

1.1 equiv.

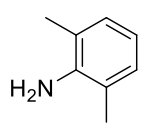

1.0 equiv.

\section{2,6-lutidine (3.1 equiv.) COMU (1.1 equiv.) solvent}

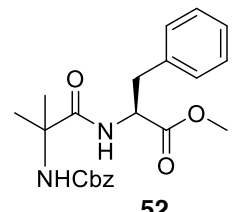

$79 \%{ }^{a}$ (2 wt\% $\mathrm{HPMC} / \mathrm{H}_{2} \mathrm{O}$ ) $79 \%^{a}\left(\mathrm{H}_{2} \mathrm{O}\right)$

2,6-lutidine ( 3.1 equiv.)
$\stackrel{\text { COMU ( } 1.1 \text { equiv.) }}{\longrightarrow \text { solvent }}$

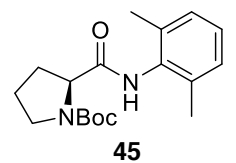

$78 \%\left(2 \mathrm{wt} \% \mathrm{HPMC} / \mathrm{H}_{2} \mathrm{O}\right)$ $20 \%{ }^{\mathrm{b}}\left(\mathrm{H}_{2} \mathrm{O}\right)$

Figure S16. Comparison between $\mathrm{HPMC} / \mathrm{H}_{2} \mathrm{O}$ and pure $\mathrm{H}_{2} \mathrm{O}$ as reaction solvents for amide couplings. General procedure (III) was followed. ${ }^{a}$ additional purification as described in section 9.1 was performed; ${ }^{b}$ additional purification via preparative HPLC was necessary due to incomplete conversion (column: Waters Xselect CSH C18, 150x30mm, $5 \mu \mathrm{m}$; eluents: Millipore water $+0.1 \%$ FA (A), MeCN + 0.1 \% FA (B); gradient: $45-60 \%$ B in $20 \mathrm{~min}, 50 \mathrm{~mL} / \mathrm{min}$ ). 


\section{Synthesis and Characterization of Four Step One-Pot Sequence}

12.1 Preparation of ethyl 3-(((S)-7-((2-ethyl-6-fluorobenzyl)oxy)chroman-3-yl)amino)2-(hydroxymethyl)propanoate (53)

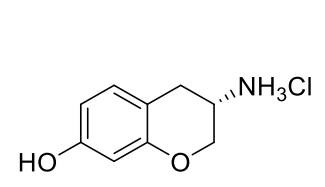

49

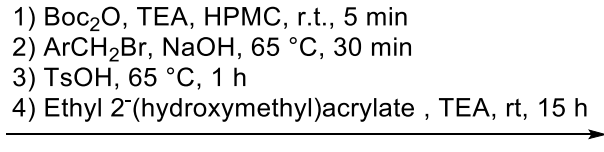

4) Ethyl 2-(hydroxymethyl)acrylate , TEA, rt, $15 \mathrm{~h}$

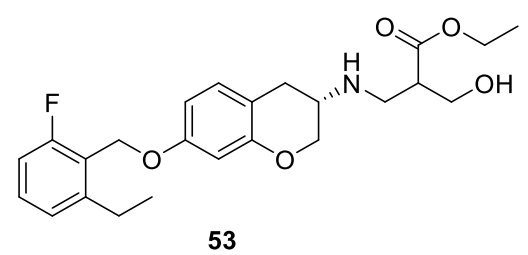

53

(S)-3-Aminochroman-7-ol hydrochloride (53) (2.48 mmol, $500 \mathrm{mg}, 1.0$ equiv.) and di-tertbutyl dicarbonate $(2.76 \mathrm{mmol}, 635 \mu$ î, 1.1 equiv.) were loaded into a $5 \mathrm{~mL}$ microwave vial open to air and containing a magnetic stir bar and Teflon-lined septum. HPMC in water solution (Mantrocel E5, $8.3 \mathrm{~mL}$ of $2 \mathrm{wt} \%$ in degassed Millipore water) was added followed by triethylamine $(2.73 \mathrm{mmol}, 382 \mu \mathrm{L}, 1.1$ equiv.). The microwave vial was closed with a septum and the reaction mixture was stirred at room temperature for 5 minutes. Completion of the reaction was confirmed by LC/MS.

To the reaction mixture was added 2-(bromomethyl)-1-ethyl-3-fluorobenzene $(2.73 \mathrm{mmol}$, $592 \mathrm{mg}, 1.1$ equiv.) and sodium hydroxide (3.22 mmol, $129 \mathrm{mg}, 1.3$ equiv.) and the suspension was stirred at $65^{\circ} \mathrm{C}$ for $15 \mathrm{~min}$. As the reaction did not go to completion an extra 1.0 equiv. of sodium hydroxide (2.45 mmol, $100 \mathrm{mg}, 1.0$ equiv.) and 0.2 equiv. of 2(bromomethyl)-1-ethyl-3- fluorobenzene ( $0.50 \mathrm{mmol}, 108 \mathrm{mg}, 0.2$ equiv.) were added and the reaction mixture was stirred at $65^{\circ} \mathrm{C}$ for an extra $15 \mathrm{~min}$. Completion of the reaction was confirmed by LC/MS.

$12 \mathrm{~N} \mathrm{HCl}$ was added dropwise to adjust the $\mathrm{pH}$ of the mixture to 1. $p$-Toluenesulfonic acid (9.92 mmol, $1.71 \mathrm{~g}, 4.00$ equiv.) was added to the mixture in two portions. The mixture was then vigorously stirred and heated at $65{ }^{\circ} \mathrm{C}$ for $15 \mathrm{~min}$. As no reaction was observed after $15 \mathrm{~min}$ extra $p$-toluenesulfonic acid (4.96 mmol, $850 \mathrm{mg}, 2.00$ equiv.) was added and the reaction was complete after $1 \mathrm{~h}$.

The mixture was cooled to room temperature and triethylamine $(12.40 \mathrm{mmol}, 1.74 \mathrm{~mL}, 5.0$ equiv.) was added in order to adjust the $\mathrm{pH}$ to 9. Ethyl 2-(hydroxymethyl)acrylate (2.48 
mmol, $323 \mathrm{mg}, 1.0$ equiv.) was then added and the mixture was stirred at room temperature for $12 \mathrm{~h}$. LCMS showed that some starting material was still left. An extra 0.50 equiv. of ethyl 2-(hydroxymethyl)acrylate ( $1.24 \mathrm{mmol}, 162 \mathrm{mg}, 0.5$ equiv.) was added and the mixture was stirred for an extra $3 \mathrm{~h}$.

To the reaction mixture were added ethyl acetate and sat. aq. sodium sulfate solution. The mixture was stirred at room temperature for $10 \mathrm{~min}$ and filtered through celite to remove the solid. The solid was washed three times with ethyl acetate. The organic phase was separated from the aq. layer. The combined ethyl acetate phases were dried in vacuo to give $1.00 \mathrm{~g}$ of crude material. After column chromatography on silica gel ( $0-5 \%$ dichloromethane/methanol in the presence of $1 \%$ triethylamine) the product $\mathbf{5 3}$ was obtained as a colorless oil (1.88 mmol, $810 \mathrm{mg}, 76 \%)$.

${ }^{1} \mathbf{H}$ NMR (600MHz, $\left.\mathrm{CDCl}_{3}\right): \delta 7.33-7.29(\mathrm{~m}, 1 \mathrm{H}), 7.08(\mathrm{~d}, J=7.6 \mathrm{~Hz}, 1 \mathrm{H}), 7.00-6.92(\mathrm{~m}$, 2H), $6.59(\mathrm{~d}, J=8.3 \mathrm{~Hz}, 1 \mathrm{H}), 6.54(\mathrm{~s}, 1 \mathrm{H}), 5.07$ (s, 2H), 4.25-4.09 (m, 3H), 4.08-3.90 (m, $3 \mathrm{H}), 3.20(\mathrm{dt}, J=12.8,6.6 \mathrm{~Hz}, 1 \mathrm{H}), 3.23-3.07(\mathrm{~m}, 2 \mathrm{H}), 3.07-2.95(\mathrm{~m}, 1 \mathrm{H}), 2.71(\mathrm{q}, J=$ $7.6 \mathrm{~Hz}, 2 \mathrm{H}), 2.66$ (p, $J=7.6 \mathrm{~Hz}, 1 \mathrm{H}), 2.64(\mathrm{dd}, J=15.9,6.6 \mathrm{~Hz}, 2 \mathrm{H}), 1.34-1.18(\mathrm{~m}, 6 \mathrm{H})$ ppm.

${ }^{13}$ C NMR (126 MHz, $\left.\mathrm{CDCl}_{3}\right) \delta 173.1,161.5(\mathrm{~d}, J=247.4 \mathrm{~Hz}), 158.4,154.8,146.9,130.7$, $130.1(\mathrm{~d}, J=9.5 \mathrm{~Hz}), 124.4,121.3(\mathrm{~d}, J=13.5 \mathrm{~Hz}), 112.8(\mathrm{~d}, J=23.0 \mathrm{~Hz}), 112.2,108.4$, 102.3, 68.4 (d, $J=11.4 \mathrm{~Hz}), 64.5,60.9,60.4,50.6,47.7,46.4,31.1,25.5,15.5$, $14.1 \mathrm{ppm}$.

${ }^{19} \mathbf{F}$ NMR (471 MHz, $\left.\mathrm{CDCl}_{3}\right) \delta-117.83 \mathrm{ppm}$.

HRMS (ESI) $\mathrm{m} / \mathrm{z}$ calcd. for $\mathrm{C}_{24} \mathrm{H}_{31} \mathrm{FNO}_{5}\left([\mathrm{M}+\mathrm{H}]^{+}\right) 432.2186$, found 432.2187 .

IR (film) vmax 2950, 1724, 1648, 1617, 1583, 1504, 1463, 1404, 1271, 1236, 1160 , $1123,1033,993,965,852,789,735,694,680 \mathrm{~cm}^{-1}$. 


\subsection{Chromatograms of the different steps}

Step 1: Boc protection:

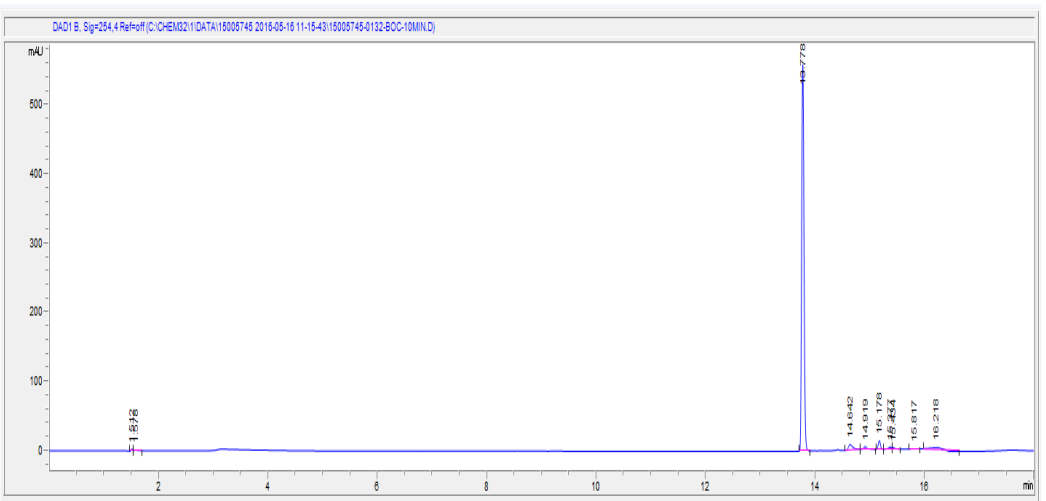

Step 2: Benzylation:

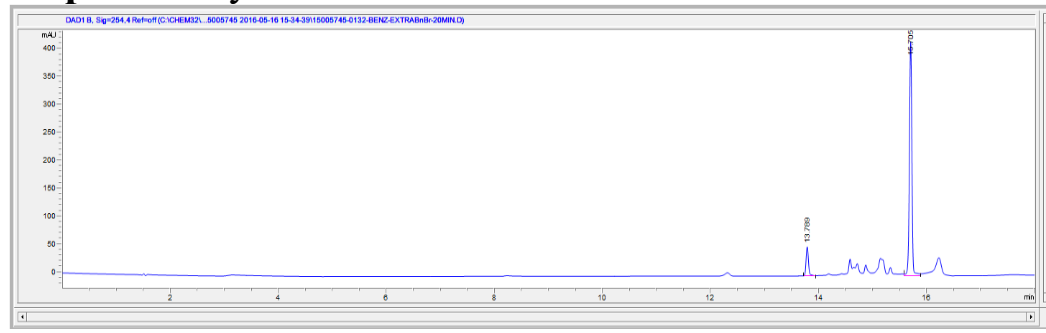

Step 3: Boc deprotection:

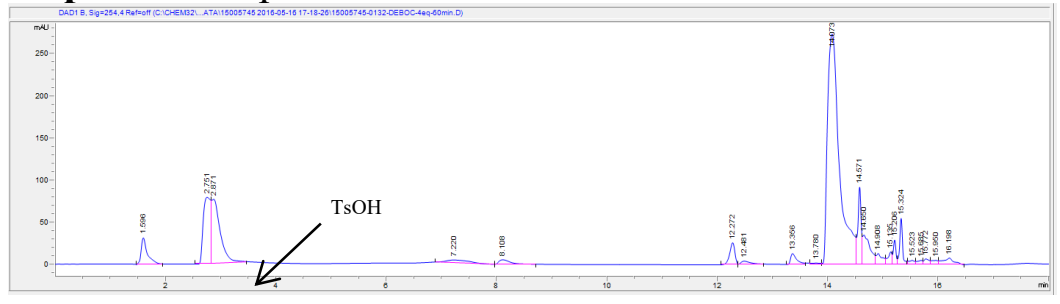

Step 4: Aza-Michael addition:

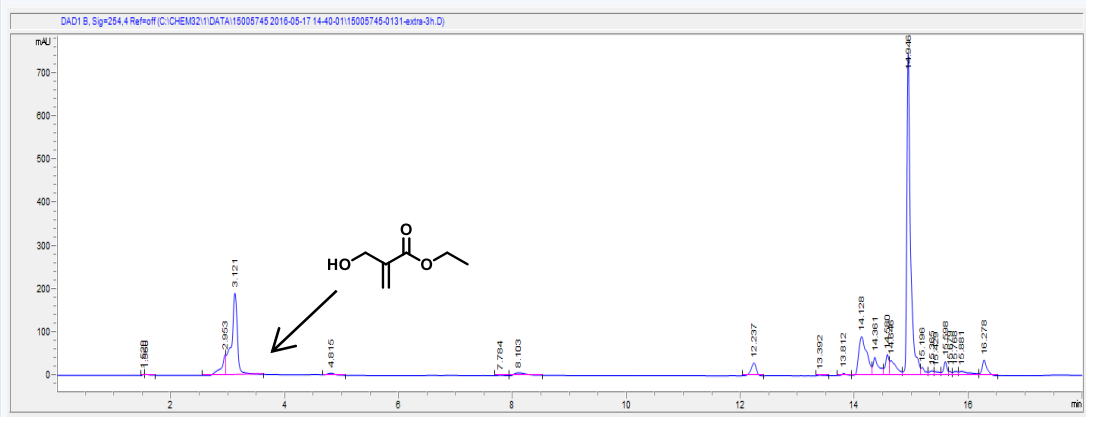


Figure S17. HPLC chromatograms of aliquots after each step of the preparation of compound 57. HPLC (Column ACE C18-AR, 100A, $4.6 \mathrm{~mm} \times 150 \mathrm{~mm}, 3.0 \mu \mathrm{m}$; UV detection at $254 \mathrm{~nm}$, eluent: $\left.10-90 \% \mathrm{MeCN} / \mathrm{H}_{2} \mathrm{O}, 1 \mathrm{~mL} / \mathrm{min}\right)$.

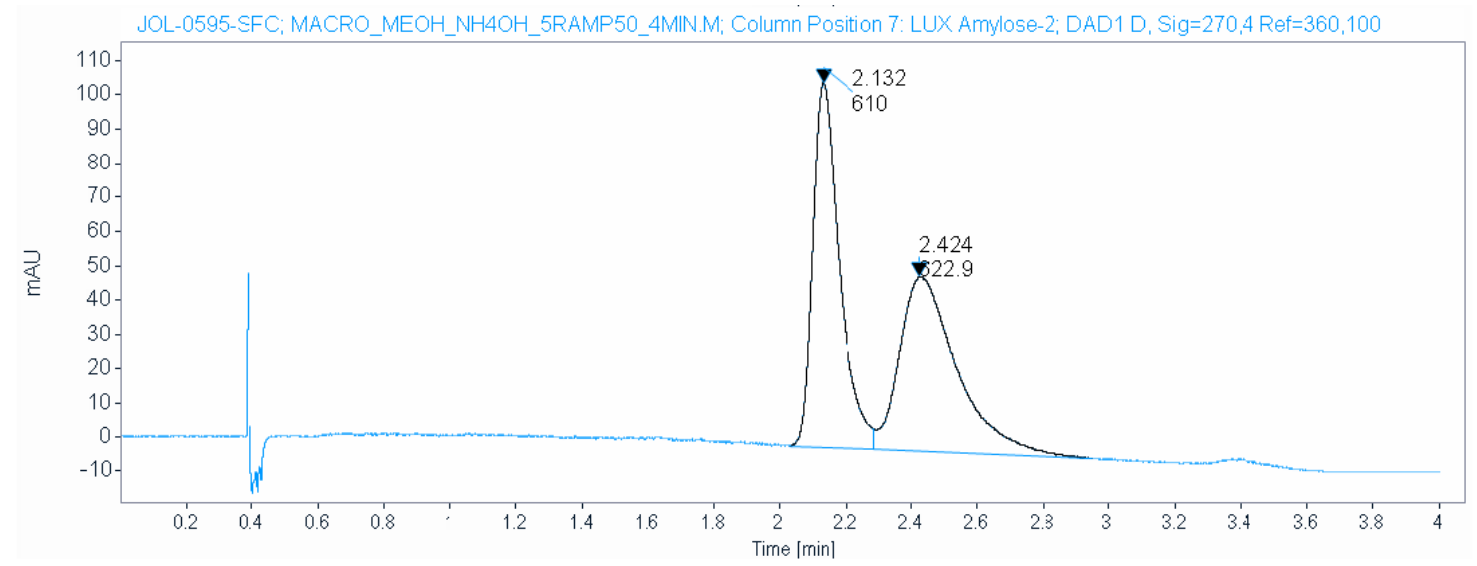

Figure S18. Separation of the diastereomers of compound 57 by chiral SFC. Column: Phenomenex LUX Amylose-2, 100x4.6 mm, $5 \mu \mathrm{m}$; eluents: $\mathrm{CO}_{2}(\mathrm{~A}), \mathrm{MeOH}+0.2 \% \mathrm{NH}_{4} \mathrm{OH}(\mathrm{B})$, gradient: $5-50 \% \mathrm{~B}$ in $3 \mathrm{~min}$, then isocratic at $50 \% \mathrm{~B}$ for $1 \mathrm{~min}, 3.5 \mathrm{~mL} / \mathrm{min}$ ).

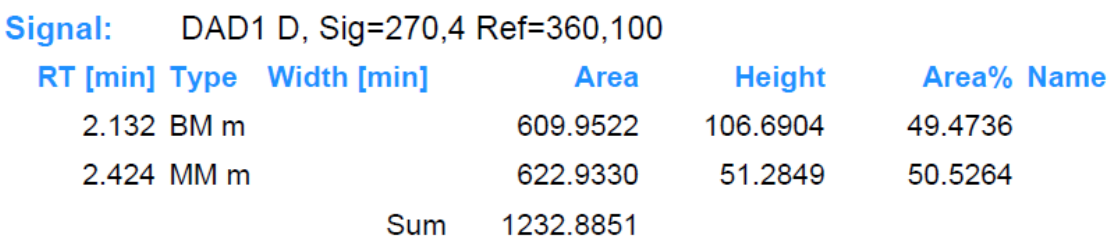




\section{Spectral Data of Buchwald-Hartwig Amination Products}

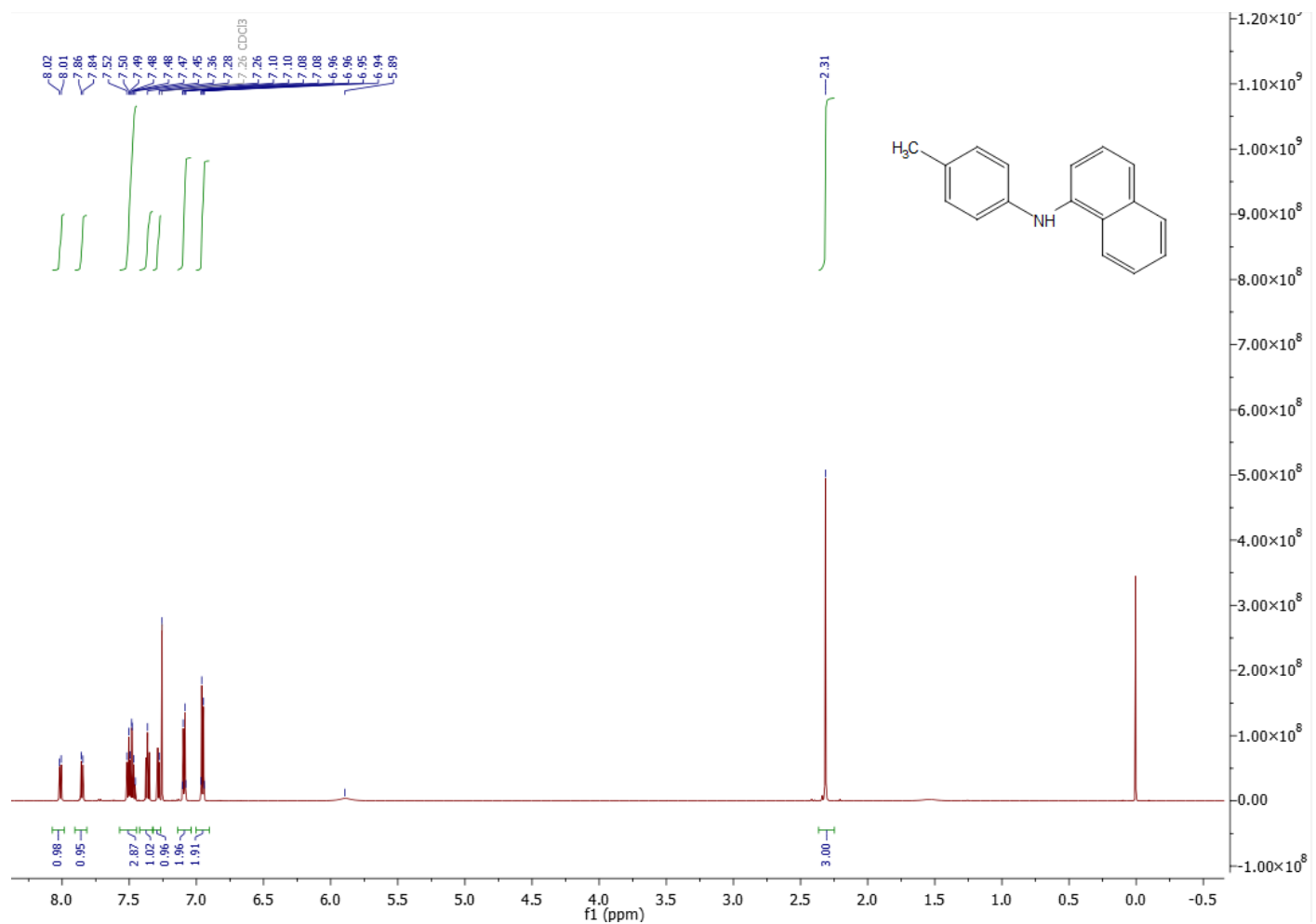

${ }^{1} \mathrm{H}$ NMR spectrum of compound 4 (500 MHz, $\left.\mathrm{CDCl}_{3}, \mathrm{rt}\right)$.

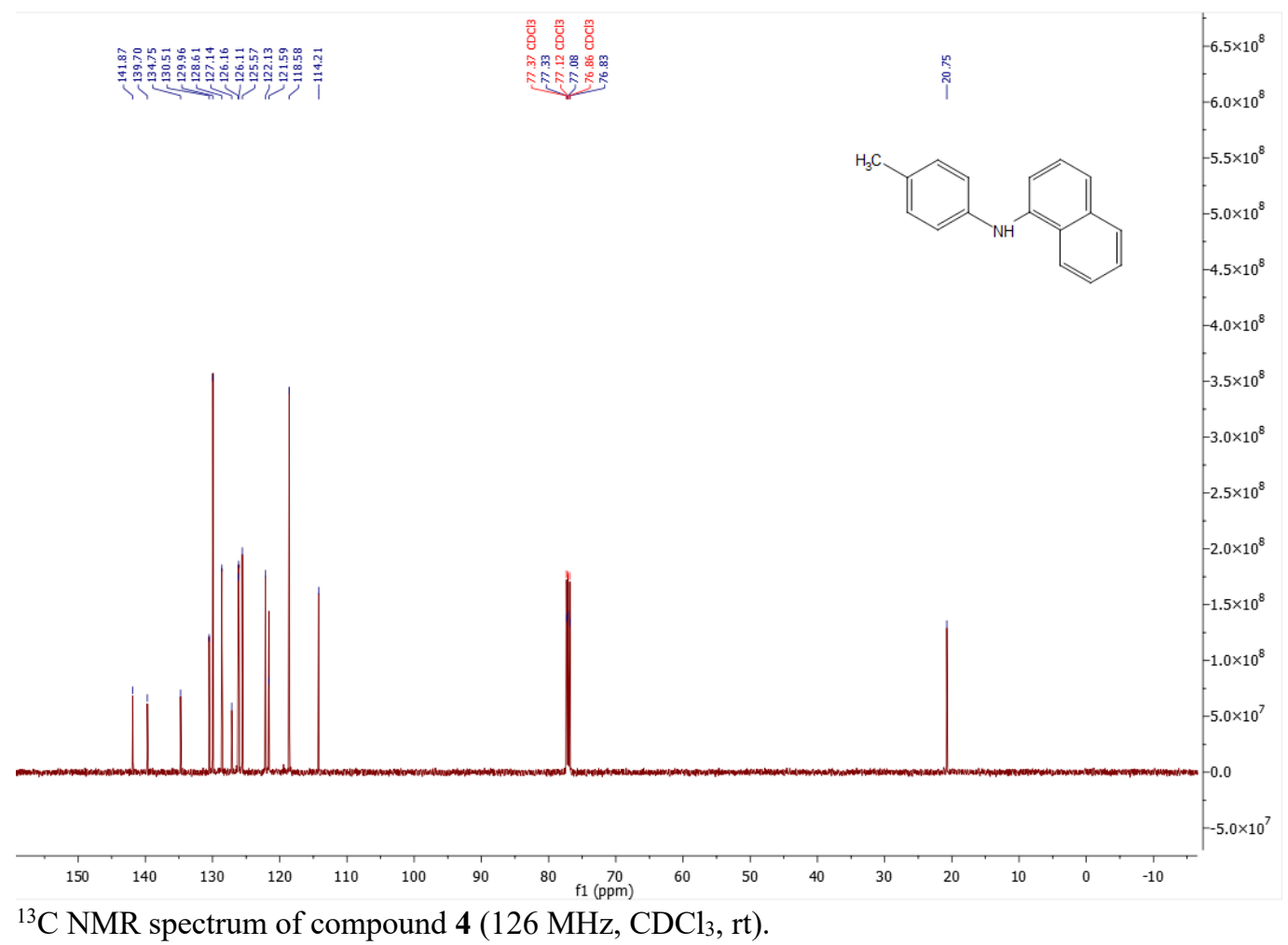




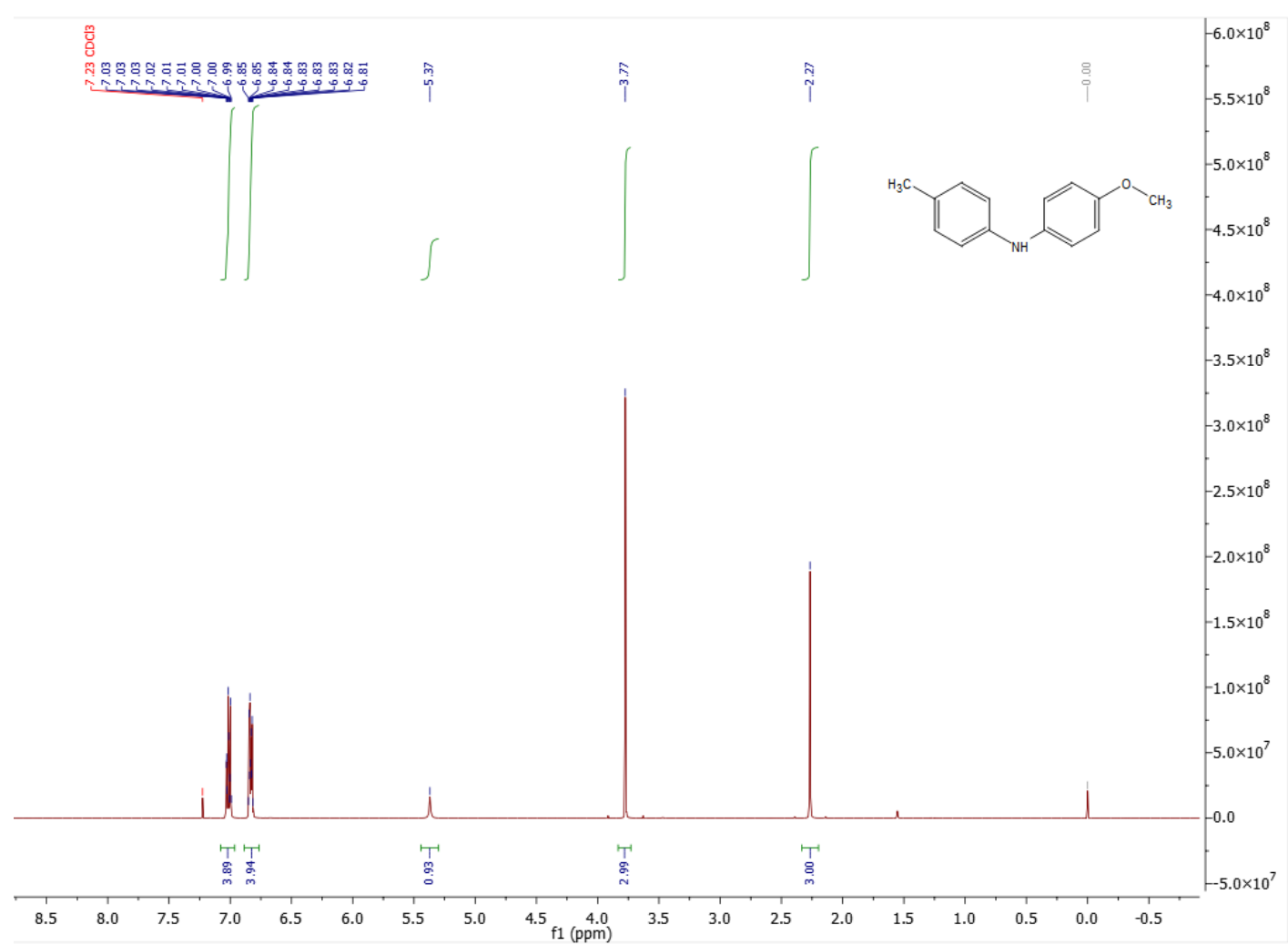

${ }^{1} \mathrm{H}$ NMR spectrum of compound $5\left(500 \mathrm{MHz}, \mathrm{CDCl}_{3}, \mathrm{rt}\right)$.

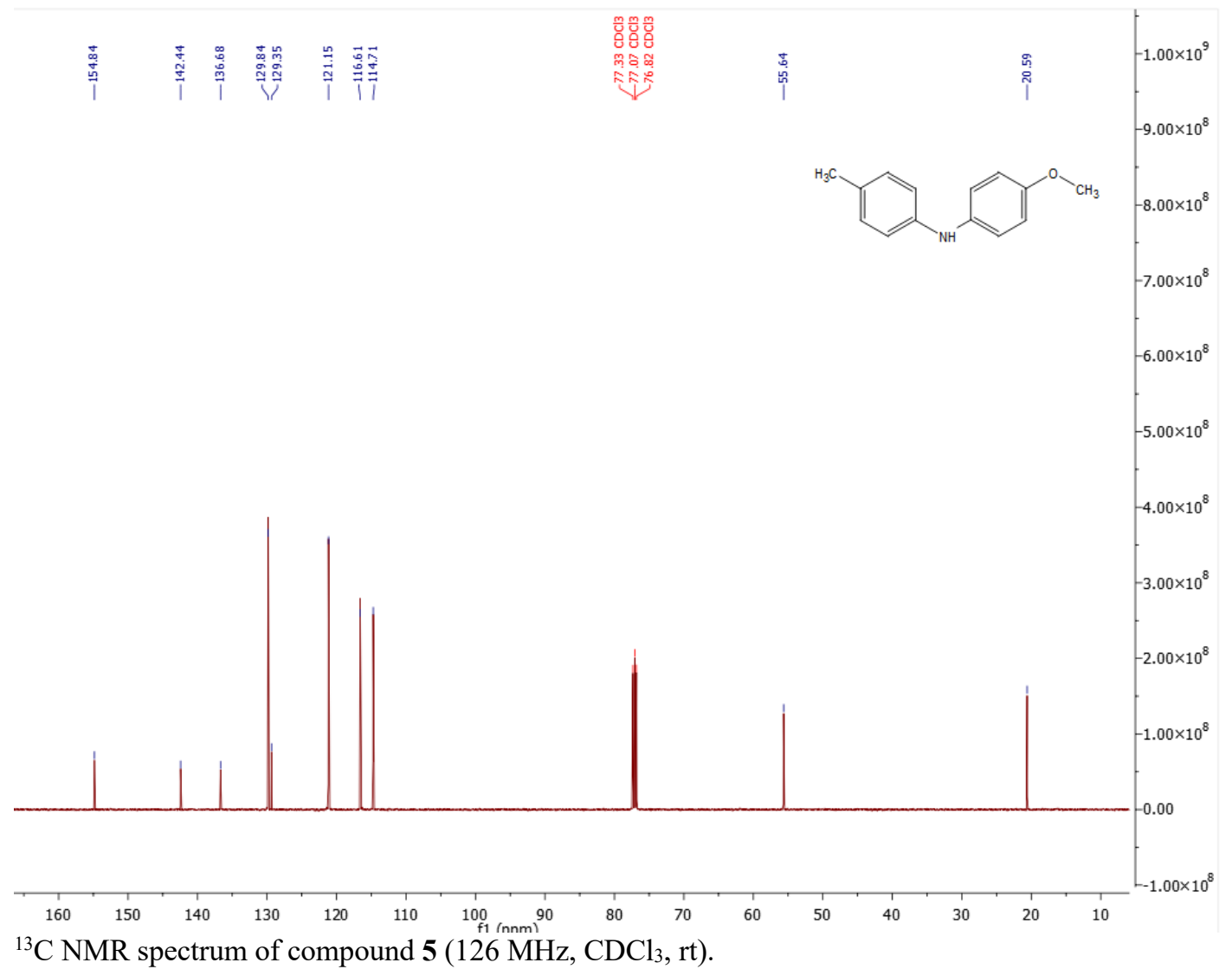




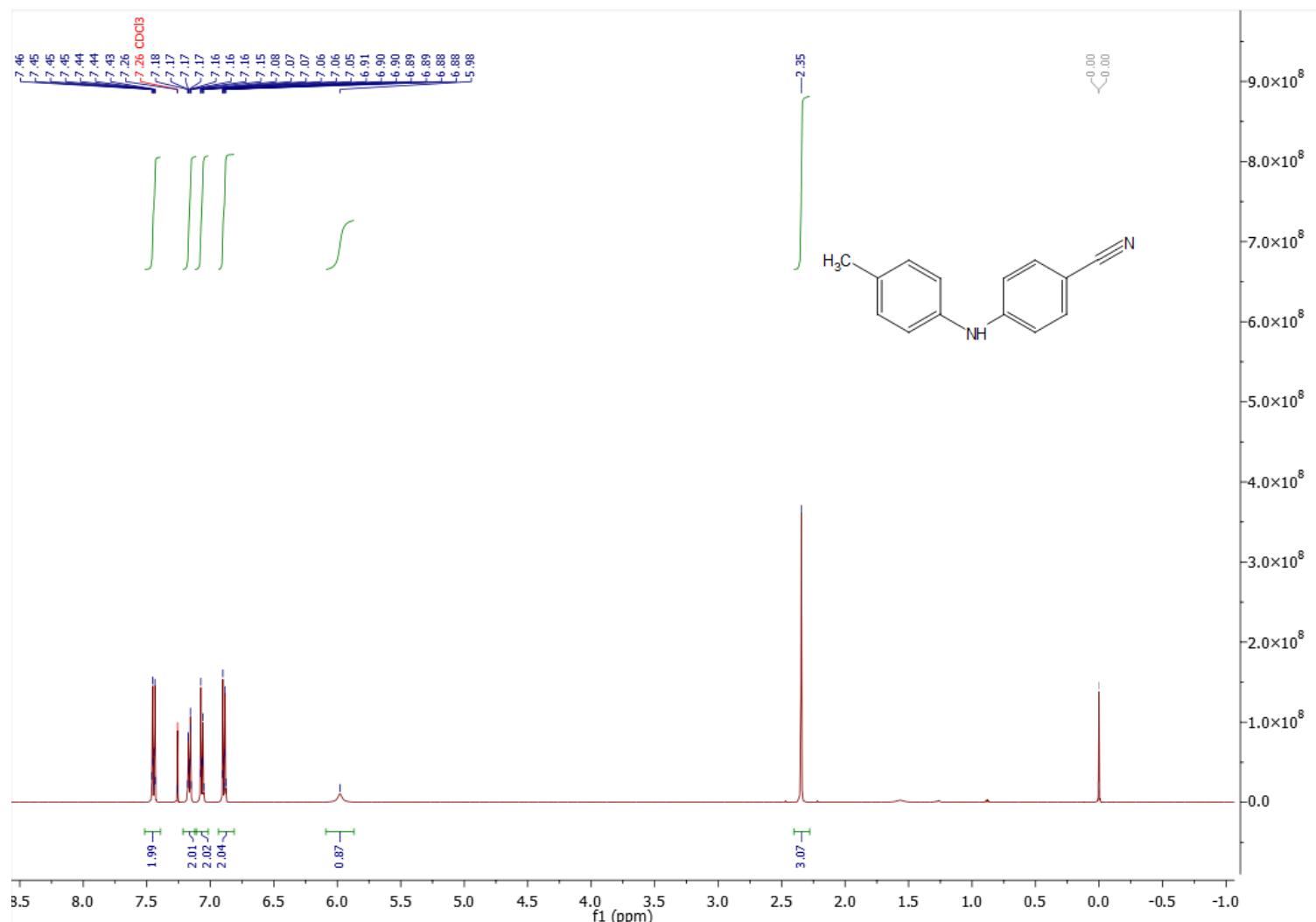

${ }^{1} \mathrm{H}$ NMR spectrum of compound 6 (500 MHz, $\mathrm{CDCl}_{3}, \mathrm{rt}$ ).

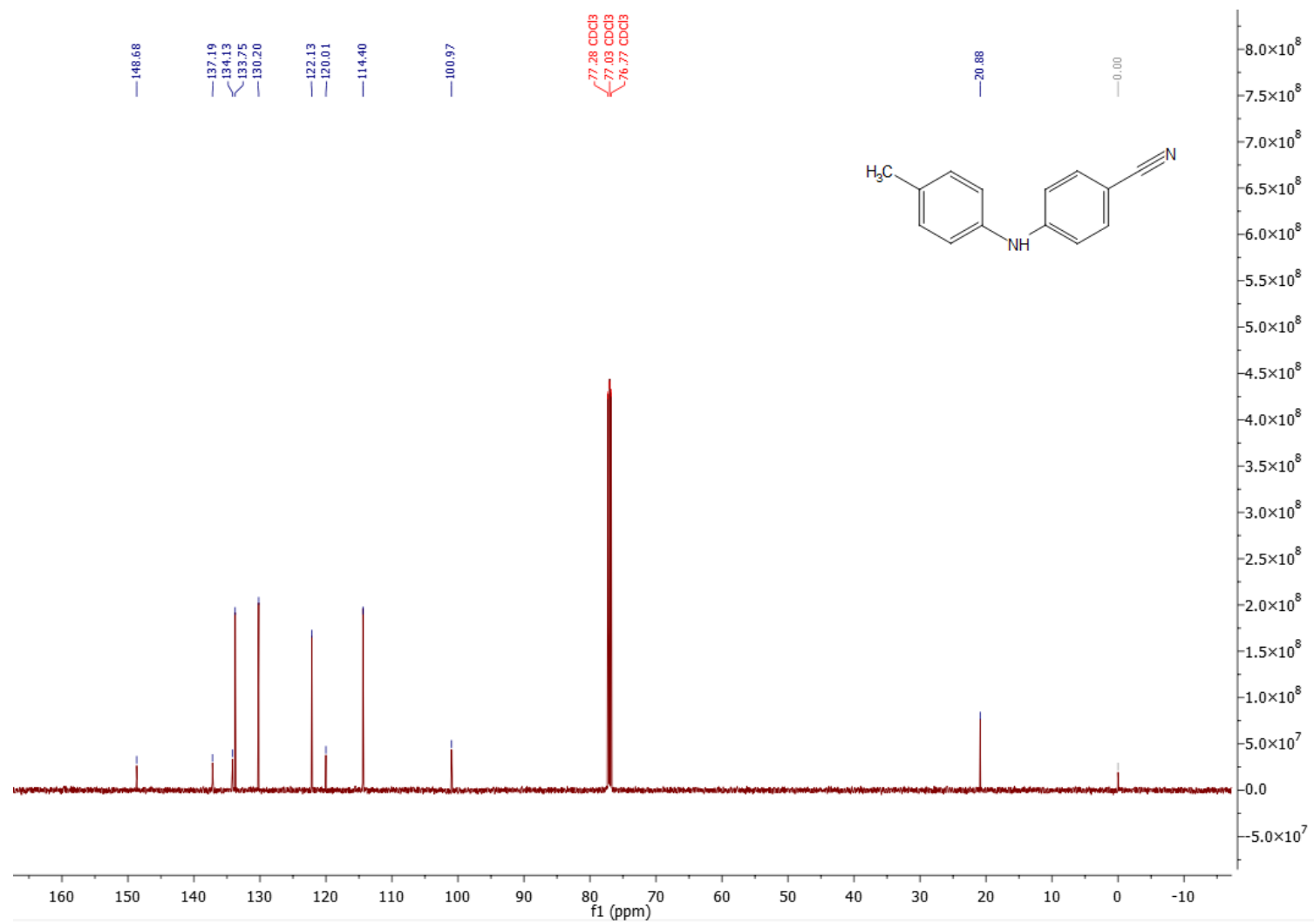

${ }^{13} \mathrm{C}$ NMR spectrum of compound $6\left(126 \mathrm{MHz}, \mathrm{CDCl}_{3}, \mathrm{rt}\right)$. 


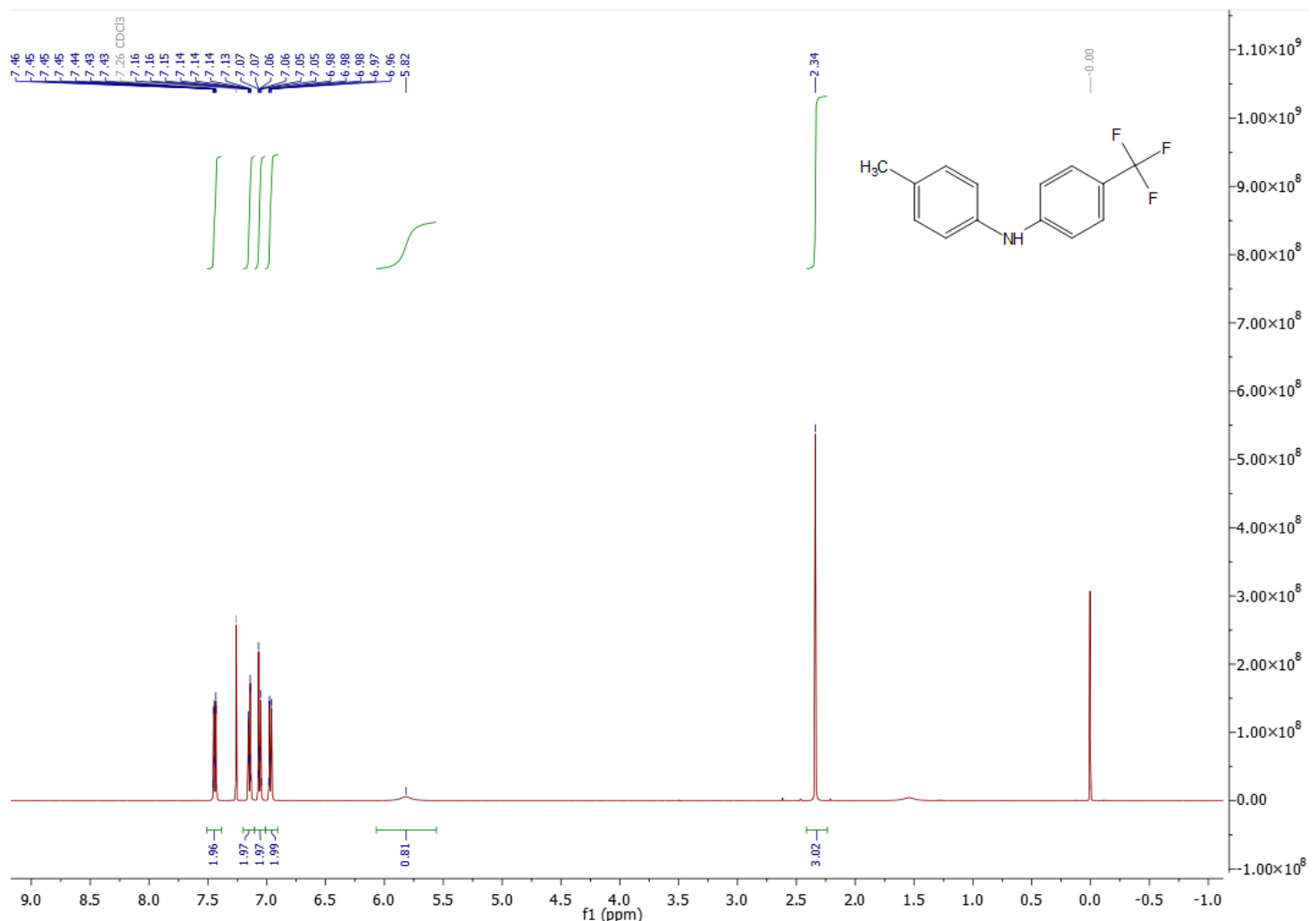

${ }^{1} \mathrm{H}$ NMR spectrum of compound 7 (500 MHz, $\mathrm{CDCl}_{3}$, rt).

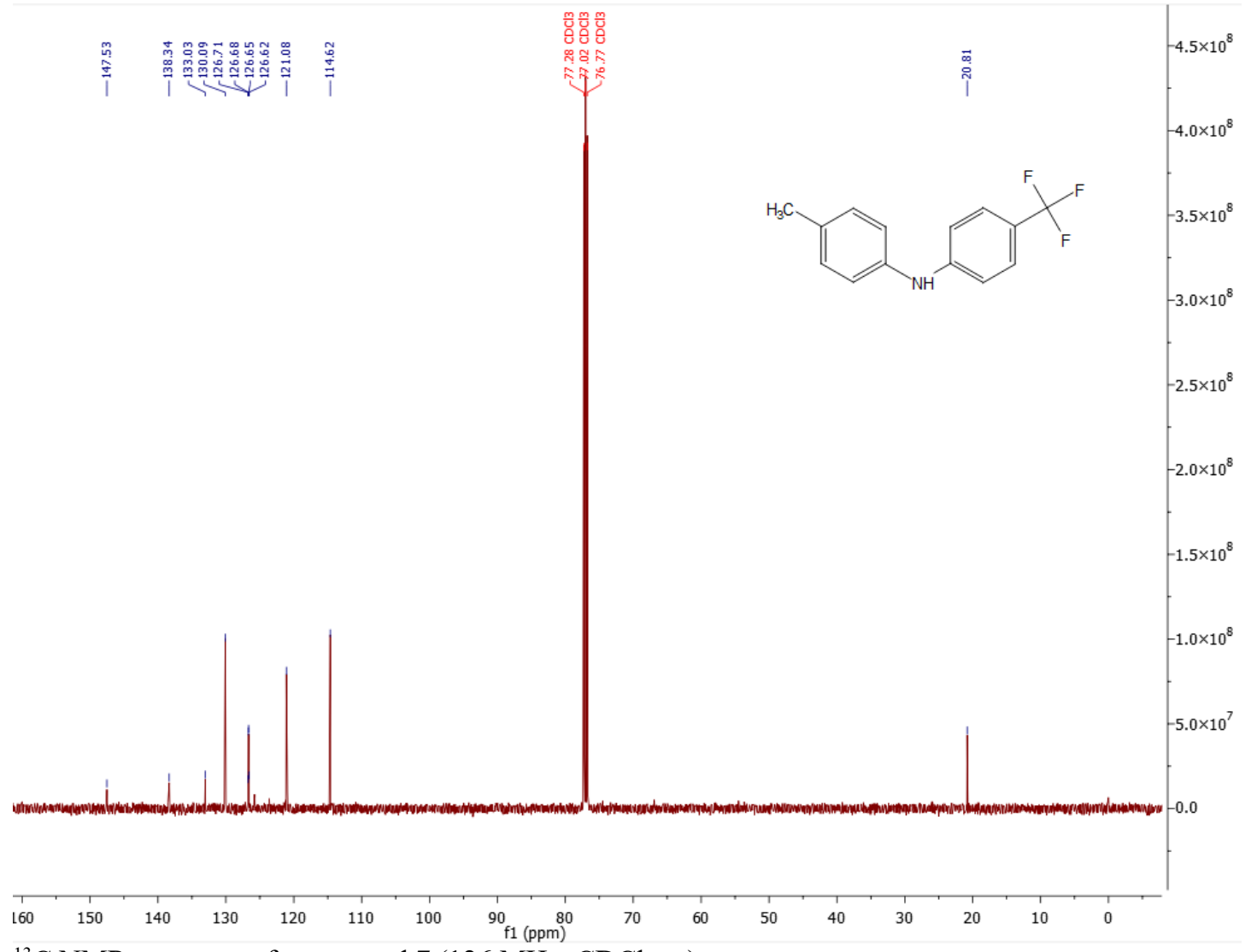

${ }^{13} \mathrm{C}$ NMR spectrum of compound 7 (126 MHz, $\left.\mathrm{CDCl}_{3}, \mathrm{rt}\right)$. 


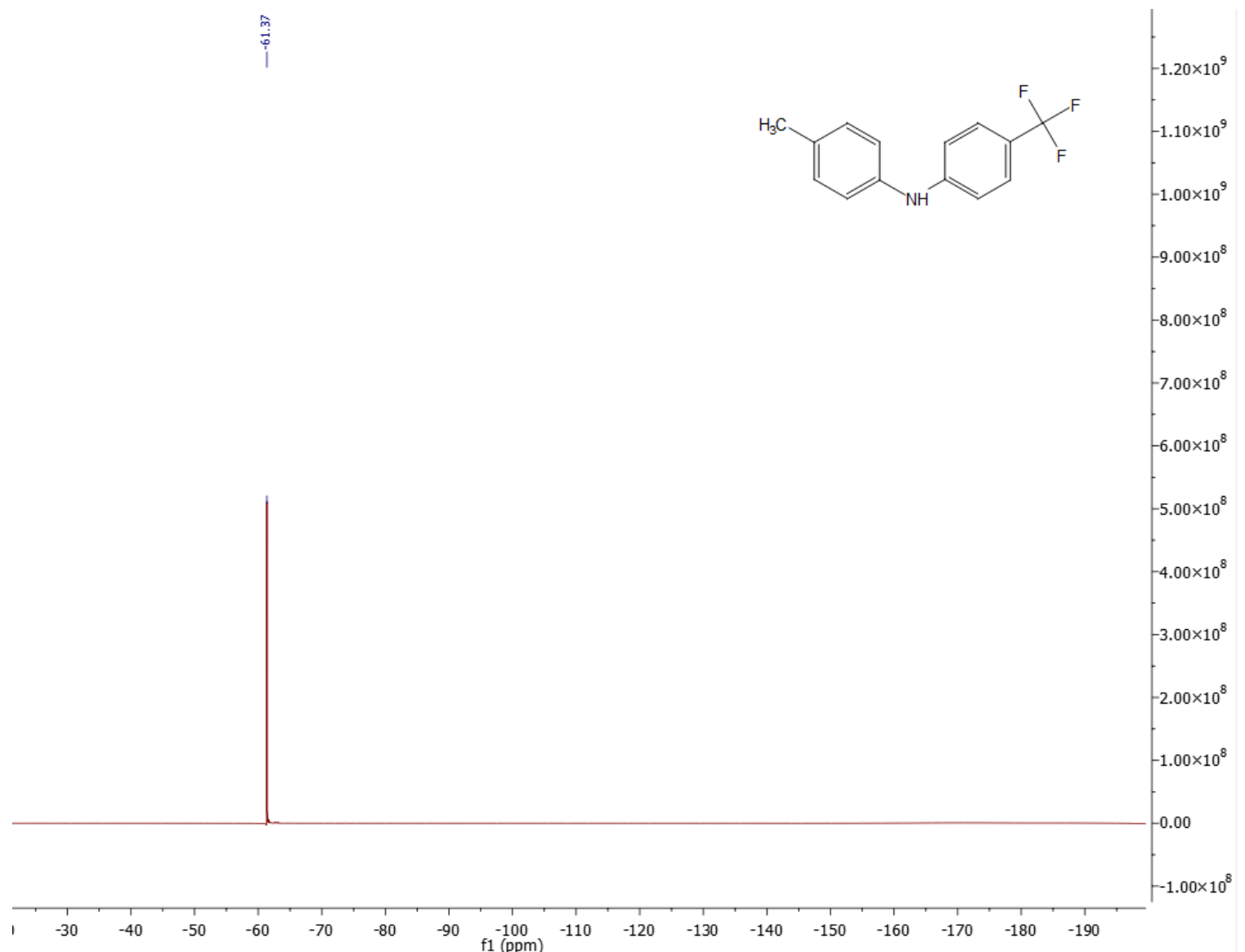

${ }^{19} \mathrm{~F}$ NMR spectrum of compound $7\left(471 \mathrm{MHz}, \mathrm{CDCl}_{3}, \mathrm{rt}\right)$. 


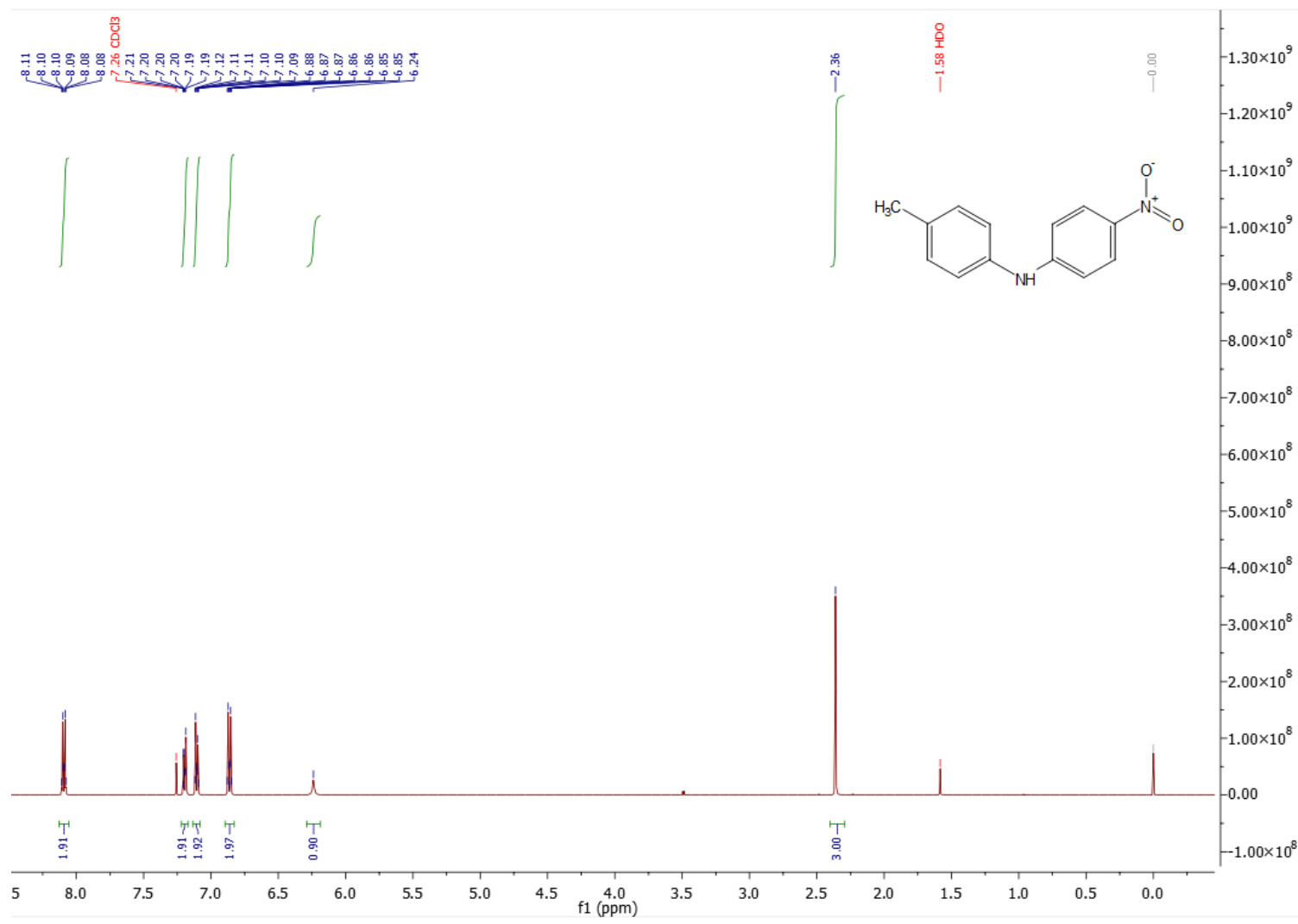

${ }^{1} \mathrm{H}$ NMR spectrum of compound 8 (500 MHz, $\left.\mathrm{CDCl}_{3}, \mathrm{rt}\right)$.

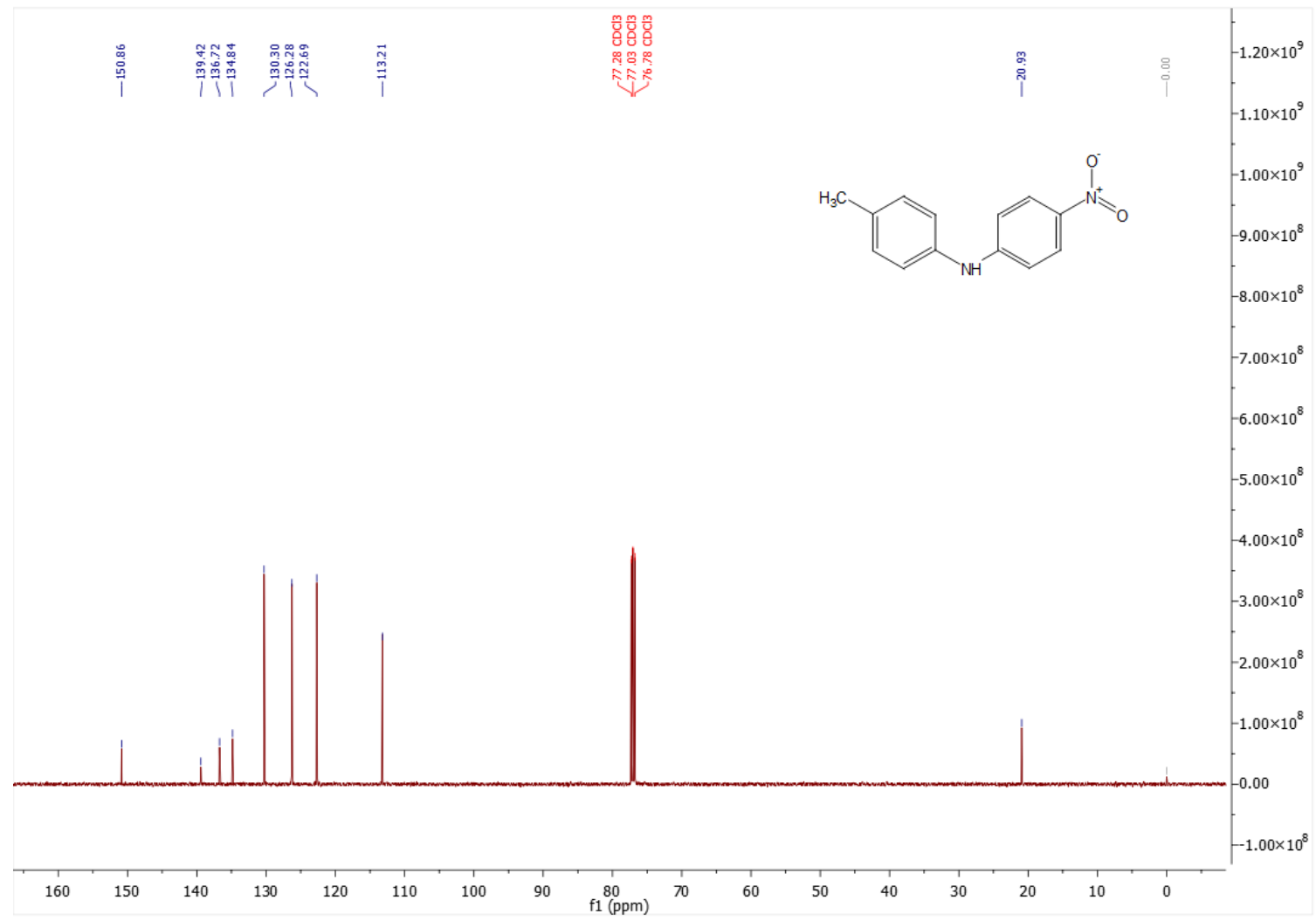

${ }^{13} \mathrm{C}$ NMR spectrum of compound $8\left(126 \mathrm{MHz}, \mathrm{CDCl}_{3}\right.$, rt $)$. 


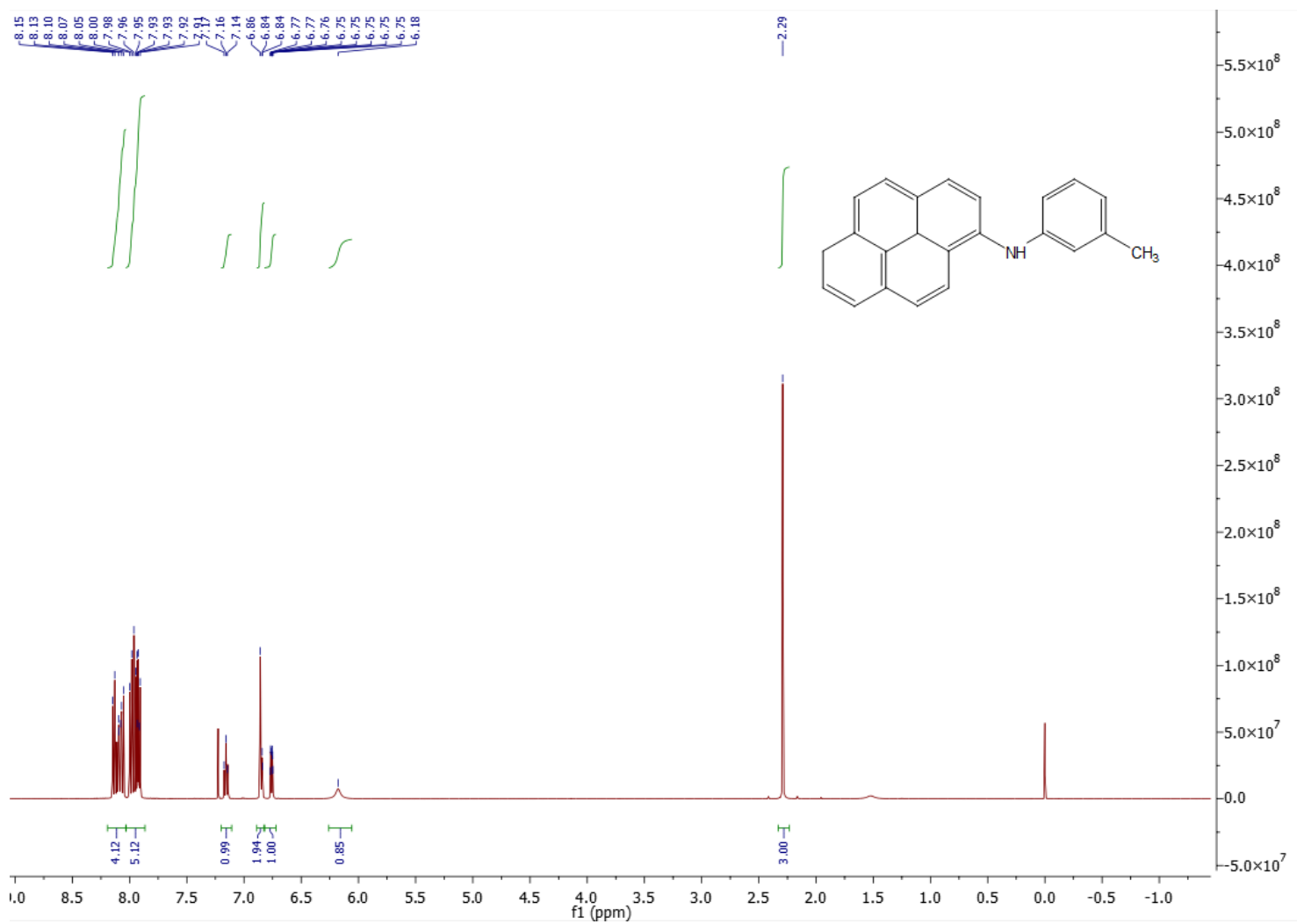

${ }^{1} \mathrm{H}$ NMR spectrum of compound $9\left(500 \mathrm{MHz}, \mathrm{CDCl}_{3}\right.$, rt).

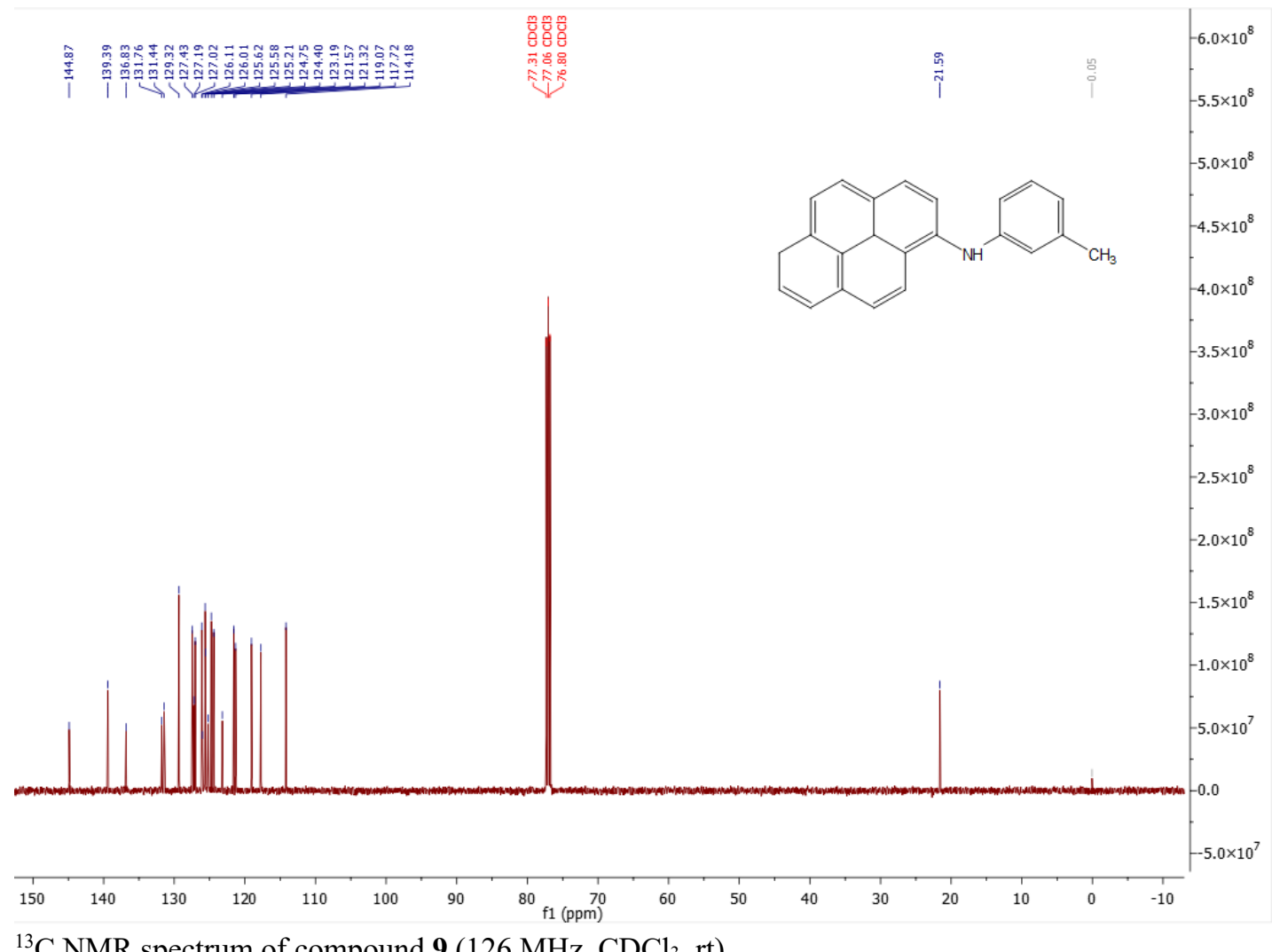

${ }^{13} \mathrm{C}$ NMR spectrum of compound $9\left(126 \mathrm{MHz}, \mathrm{CDCl}_{3}\right.$, rt). 


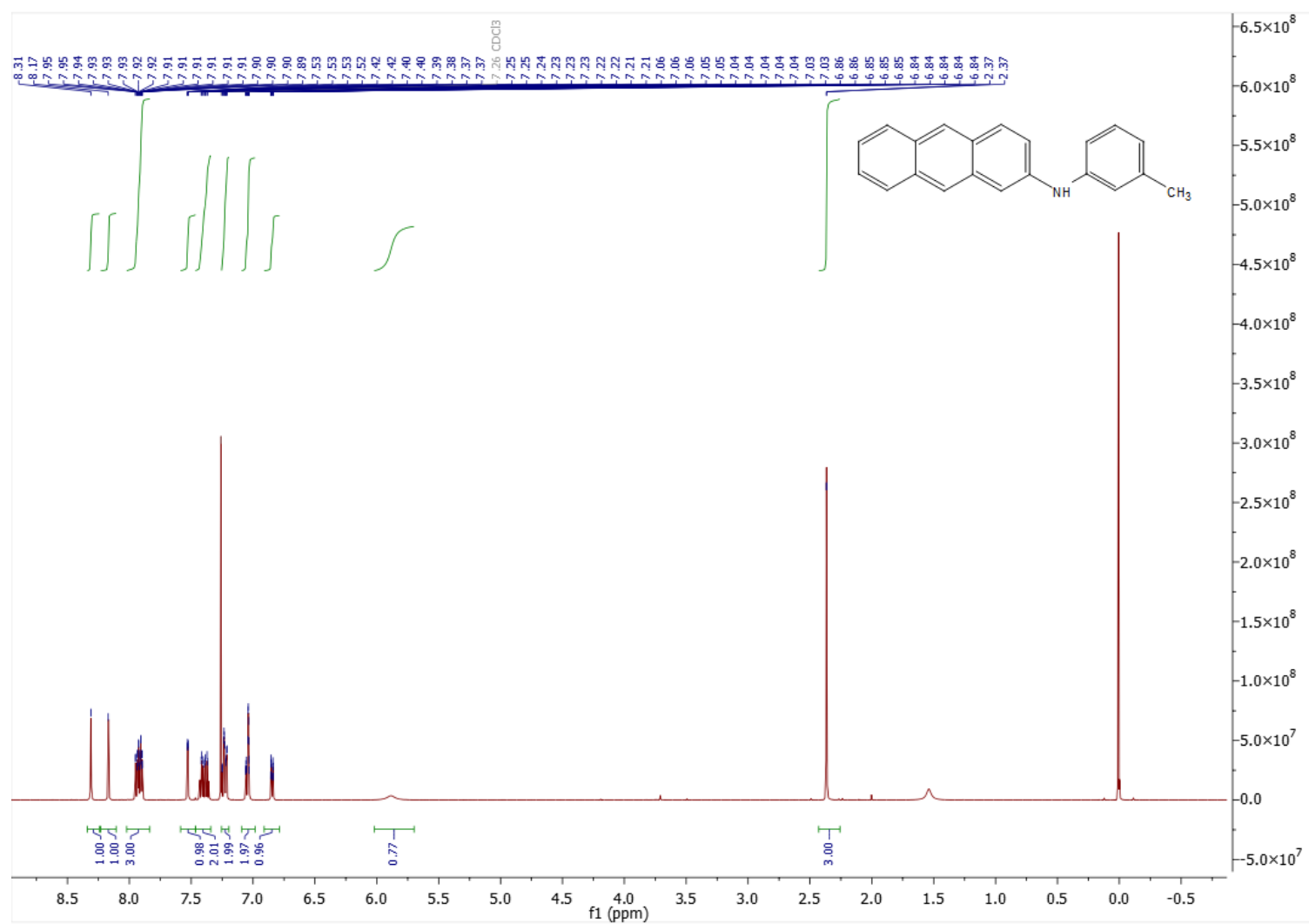

${ }^{1} \mathrm{H}$ NMR spectrum of compound $10\left(500 \mathrm{MHz}, \mathrm{CDCl}_{3}, \mathrm{rt}\right)$.

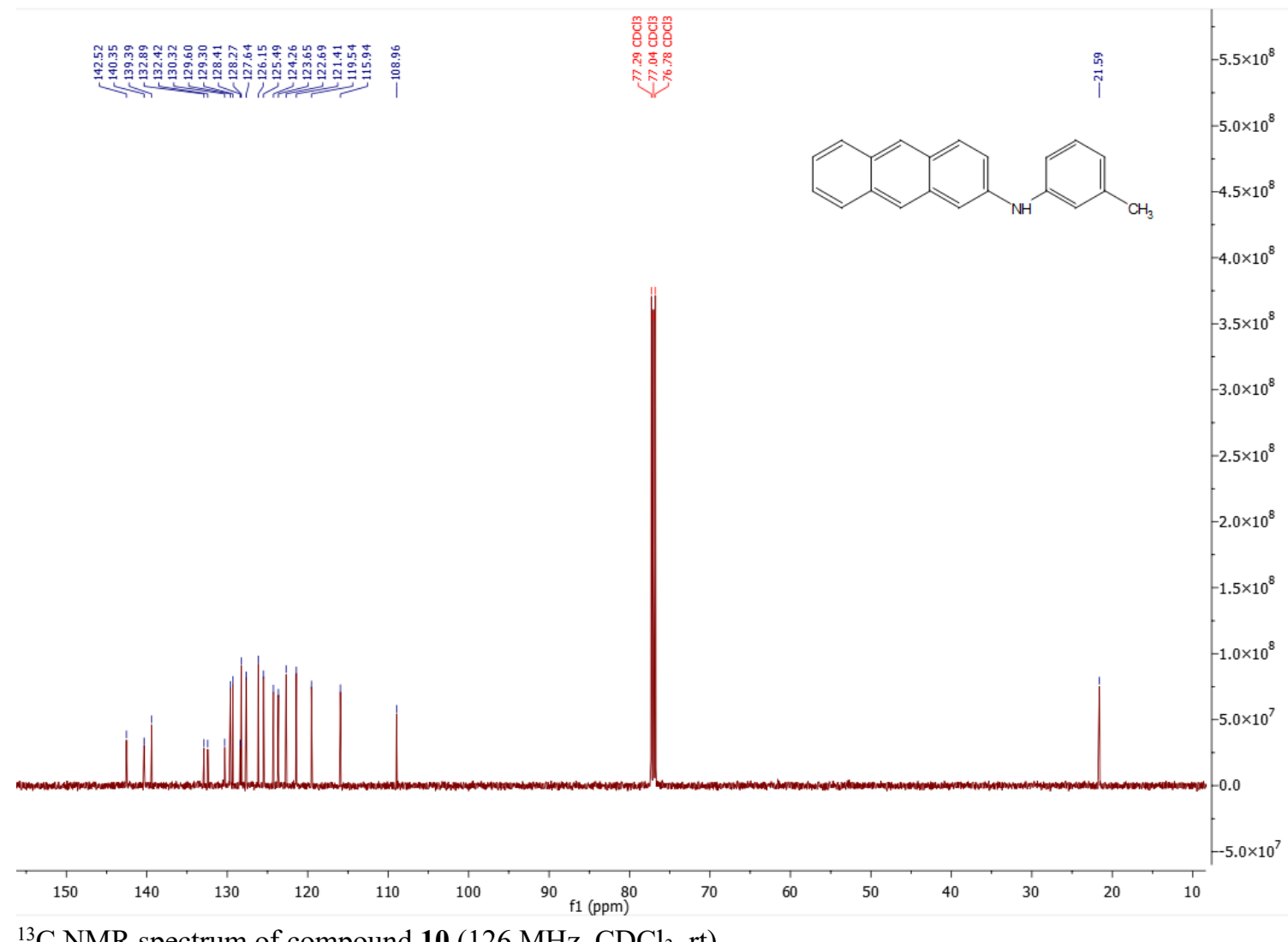

${ }^{13} \mathrm{C}$ NMR spectrum of compound $\mathbf{1 0}\left(126 \mathrm{MHz}, \mathrm{CDCl}_{3}, \mathrm{rt}\right)$. 


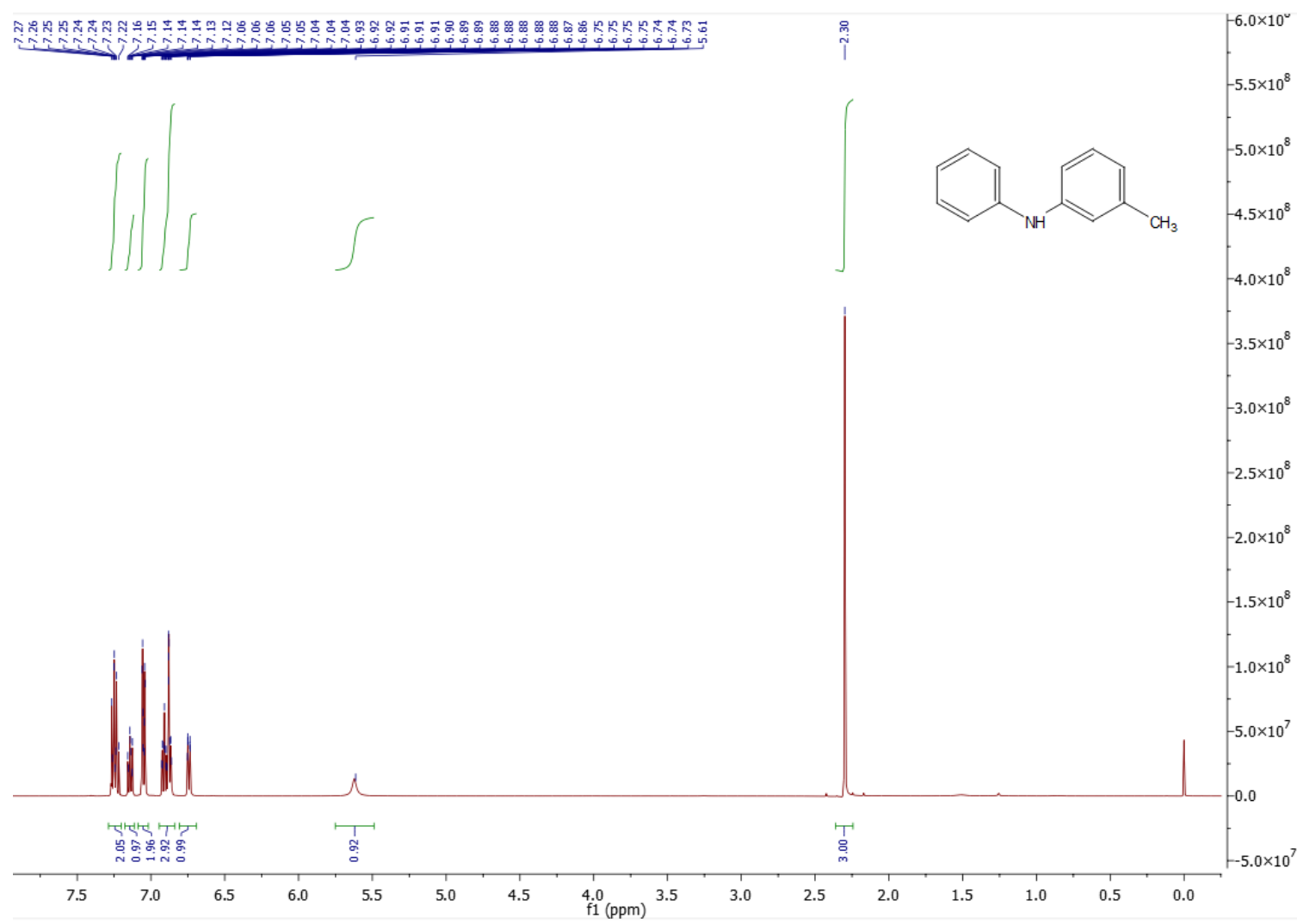

${ }^{1} \mathrm{H}$ NMR spectrum of compound $11\left(500 \mathrm{MHz}, \mathrm{CDCl}_{3}, \mathrm{rt}\right)$.

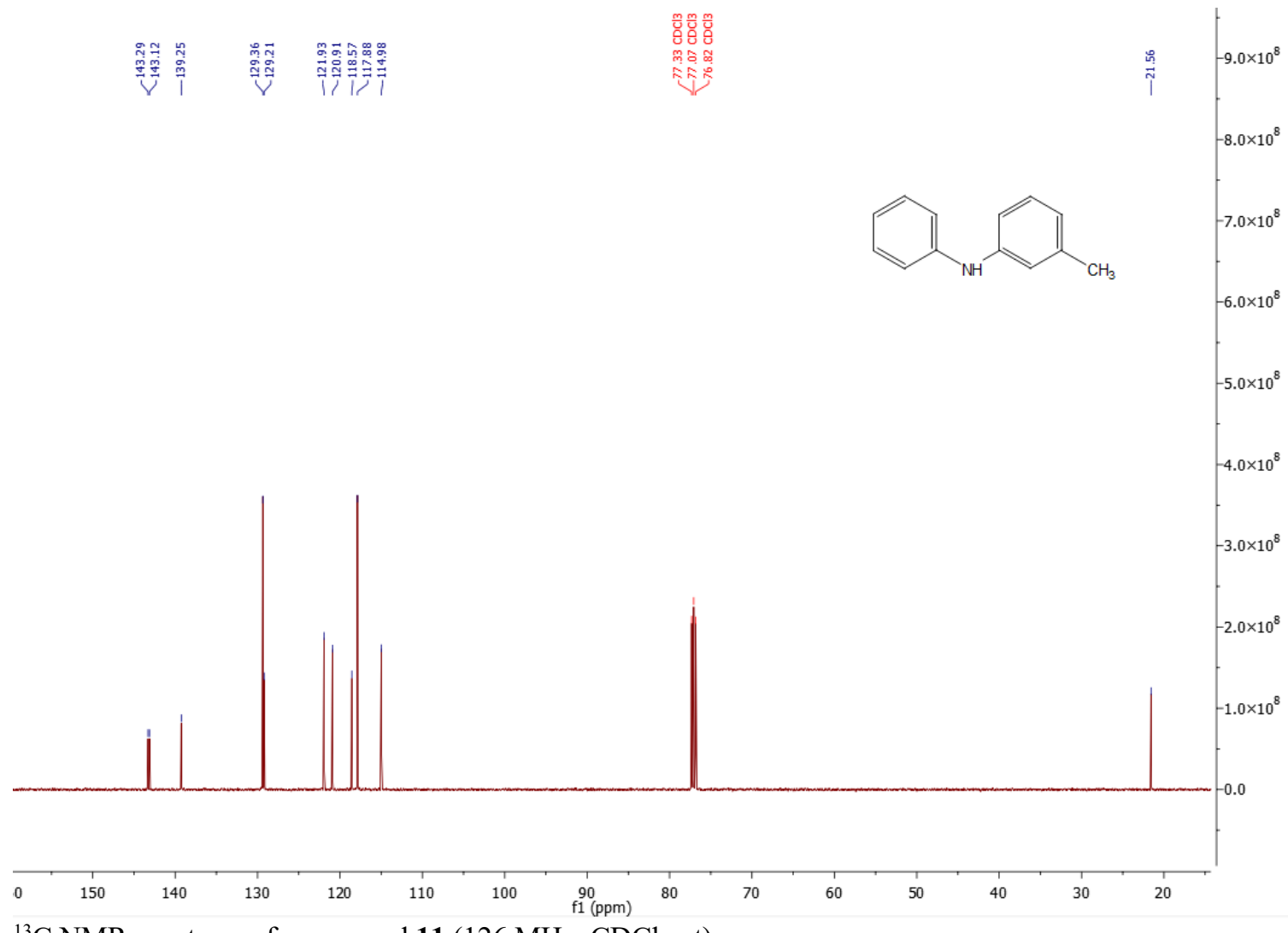

${ }^{13} \mathrm{C}$ NMR spectrum of compound $11\left(126 \mathrm{MHz}, \mathrm{CDCl}_{3}, \mathrm{rt}\right)$. 


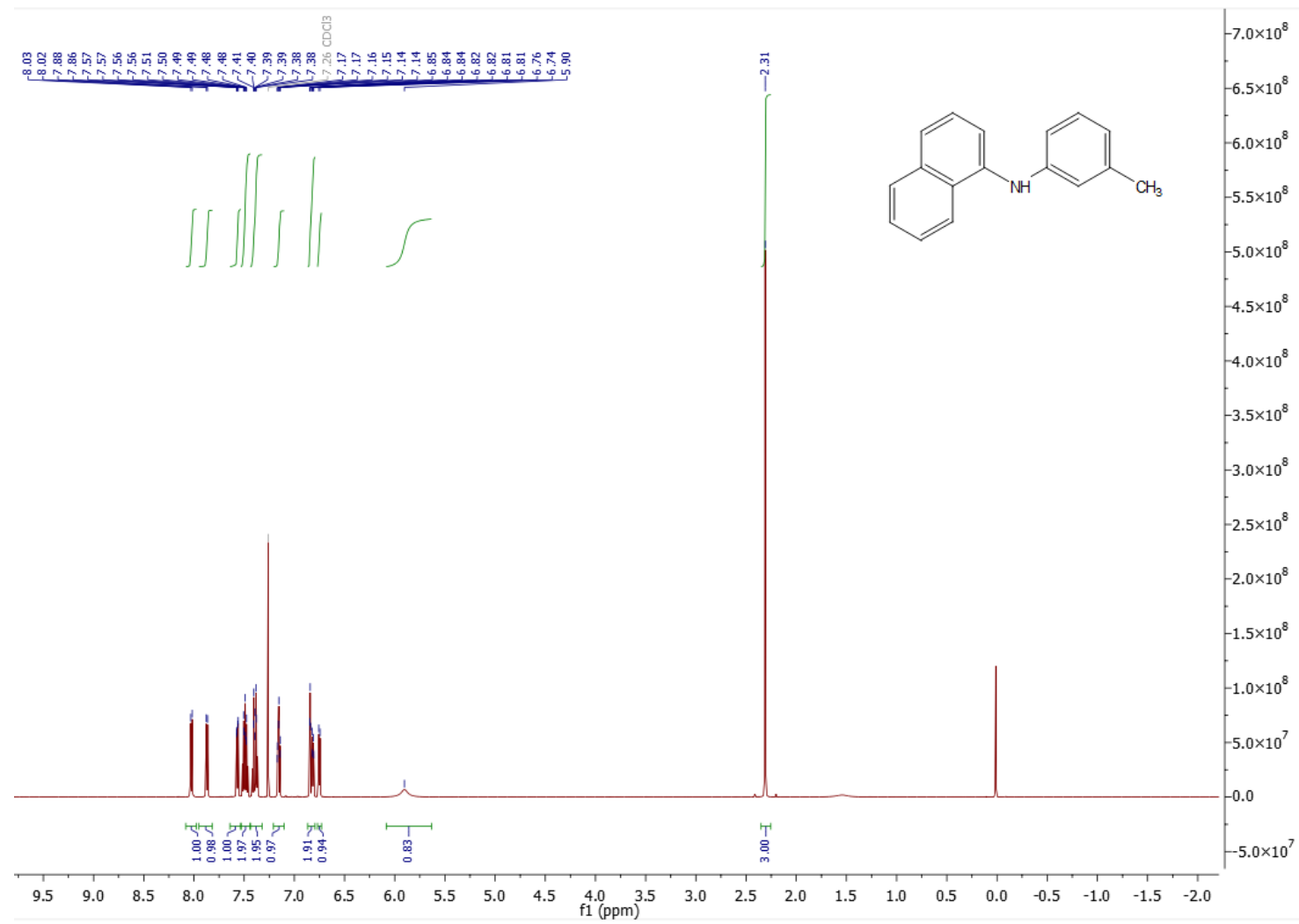

${ }^{1} \mathrm{H}$ NMR spectrum of compound $12\left(500 \mathrm{MHz}, \mathrm{CDCl}_{3}\right.$, rt).

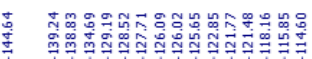

।
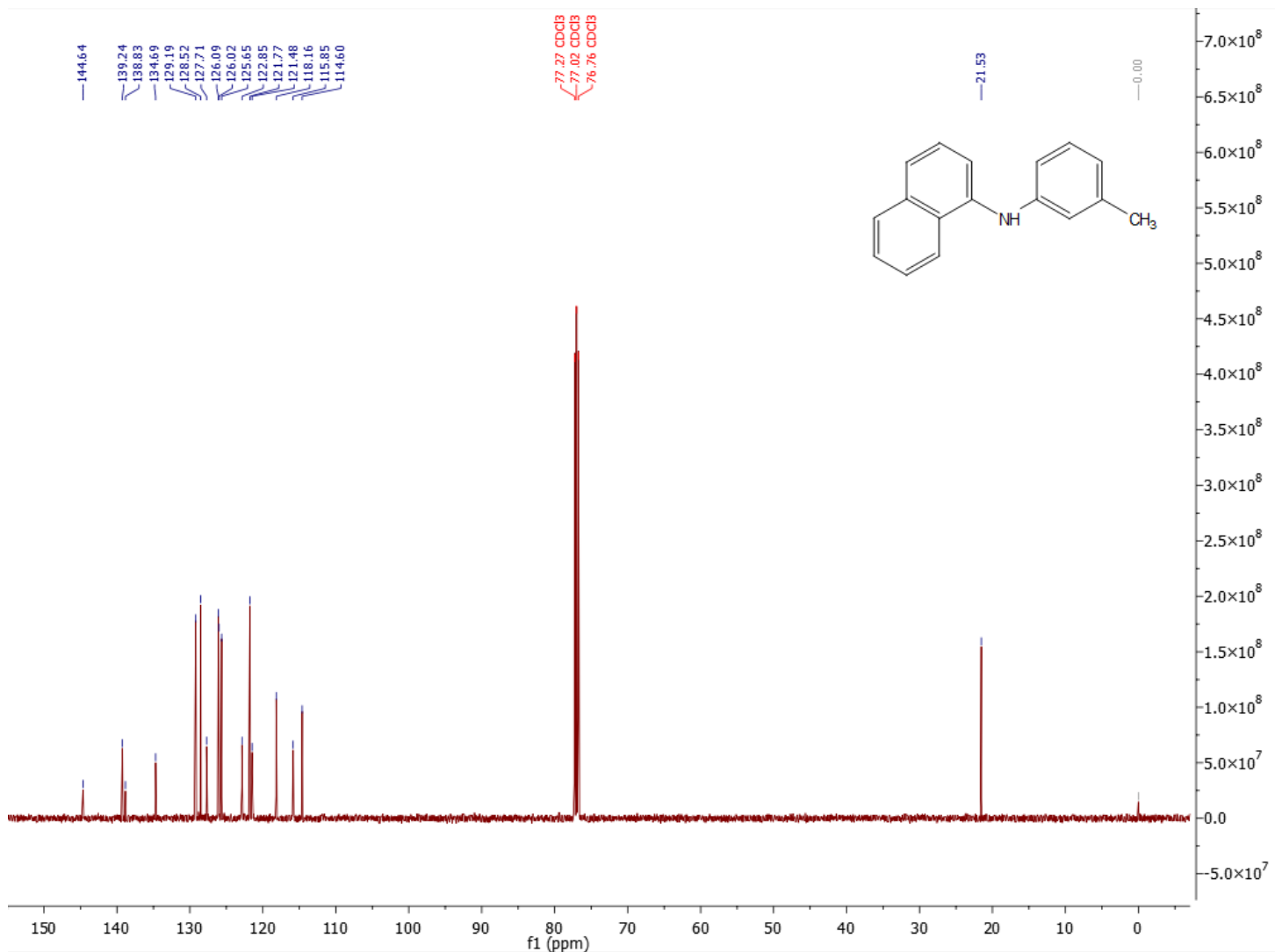

${ }^{13} \mathrm{C}$ NMR spectrum of compound $12\left(126 \mathrm{MHz}, \mathrm{CDCl}_{3}, \mathrm{rt}\right)$. 


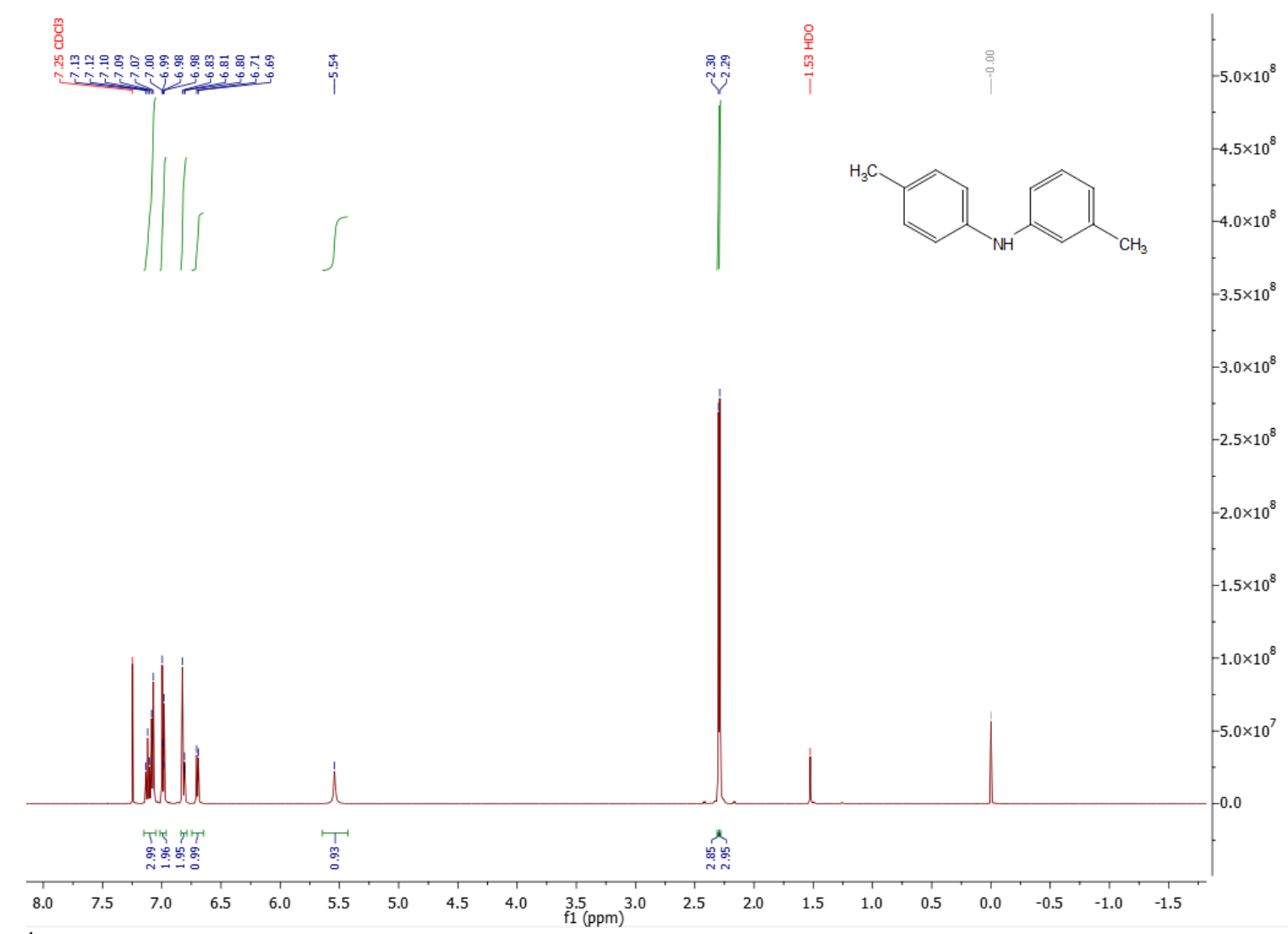

${ }^{1} \mathrm{H}$ NMR spectrum of compound $13\left(500 \mathrm{MHz}, \mathrm{CDCl}_{3}, \mathrm{rt}\right)$.

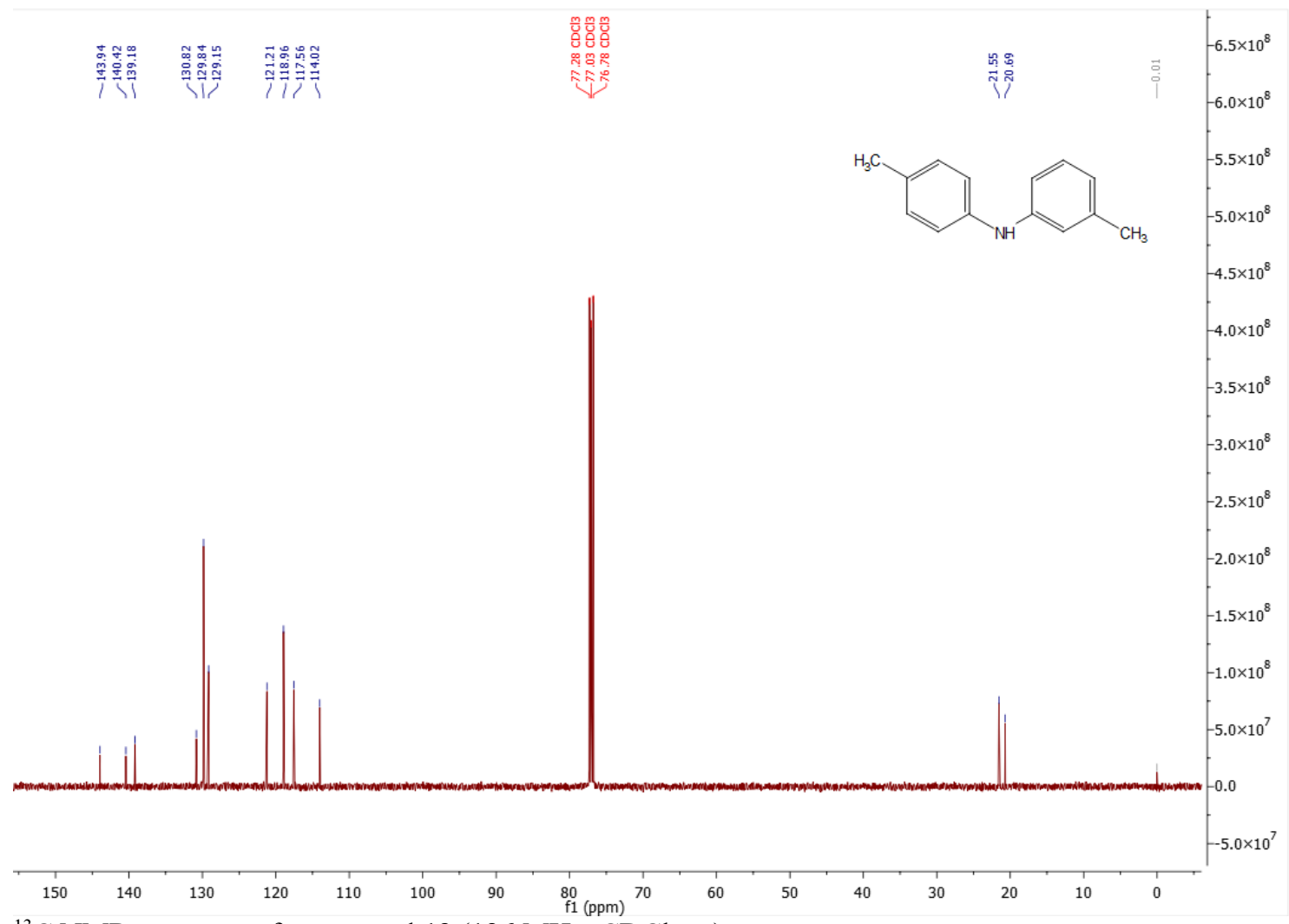

${ }^{13} \mathrm{C}$ NMR spectrum of compound $13\left(126 \mathrm{MHz}, \mathrm{CDCl}_{3}, \mathrm{rt}\right)$. 


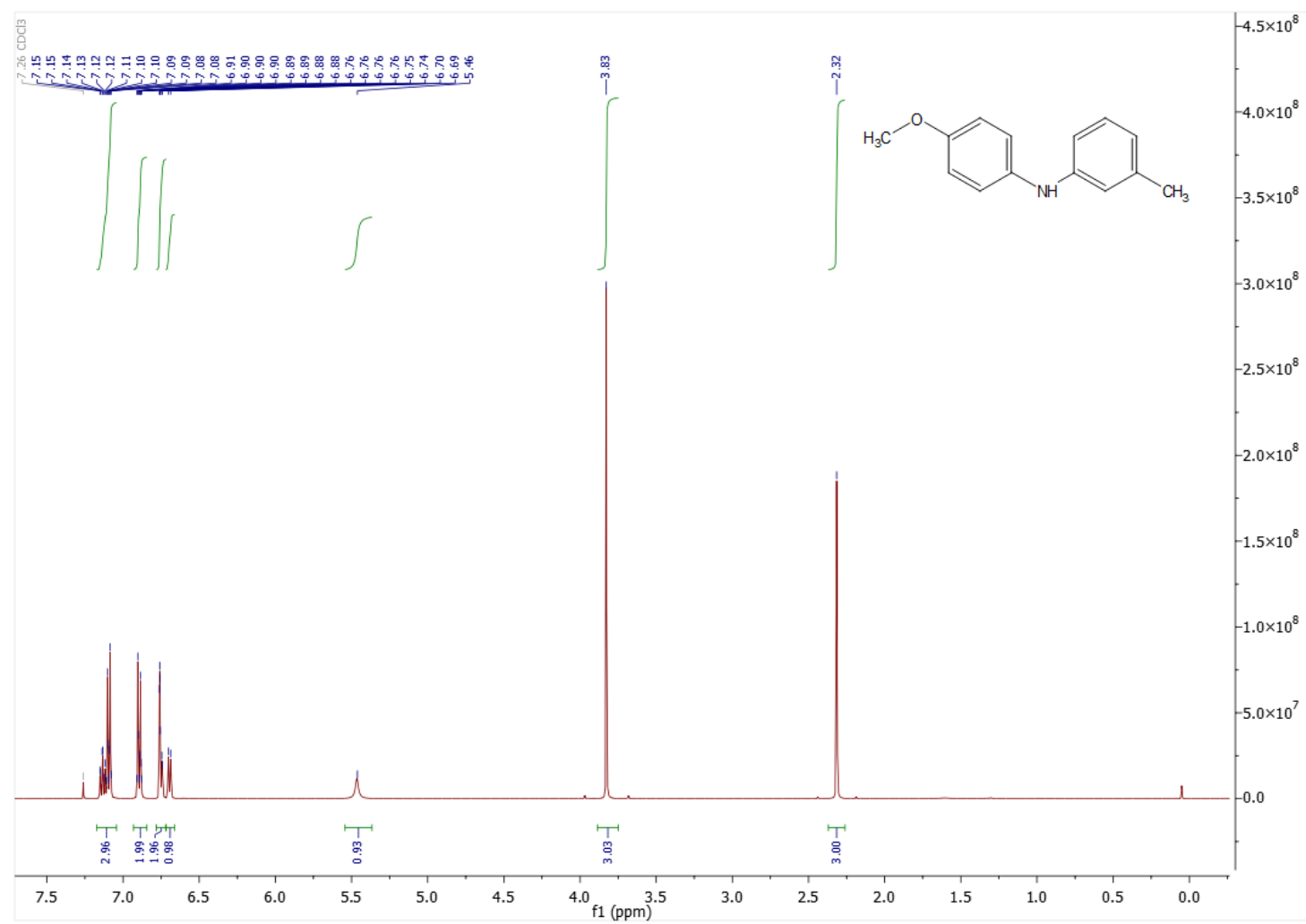

${ }^{1} \mathrm{H}$ NMR spectrum of compound $14\left(500 \mathrm{MHz}, \mathrm{CDCl}_{3}, \mathrm{rt}\right)$.

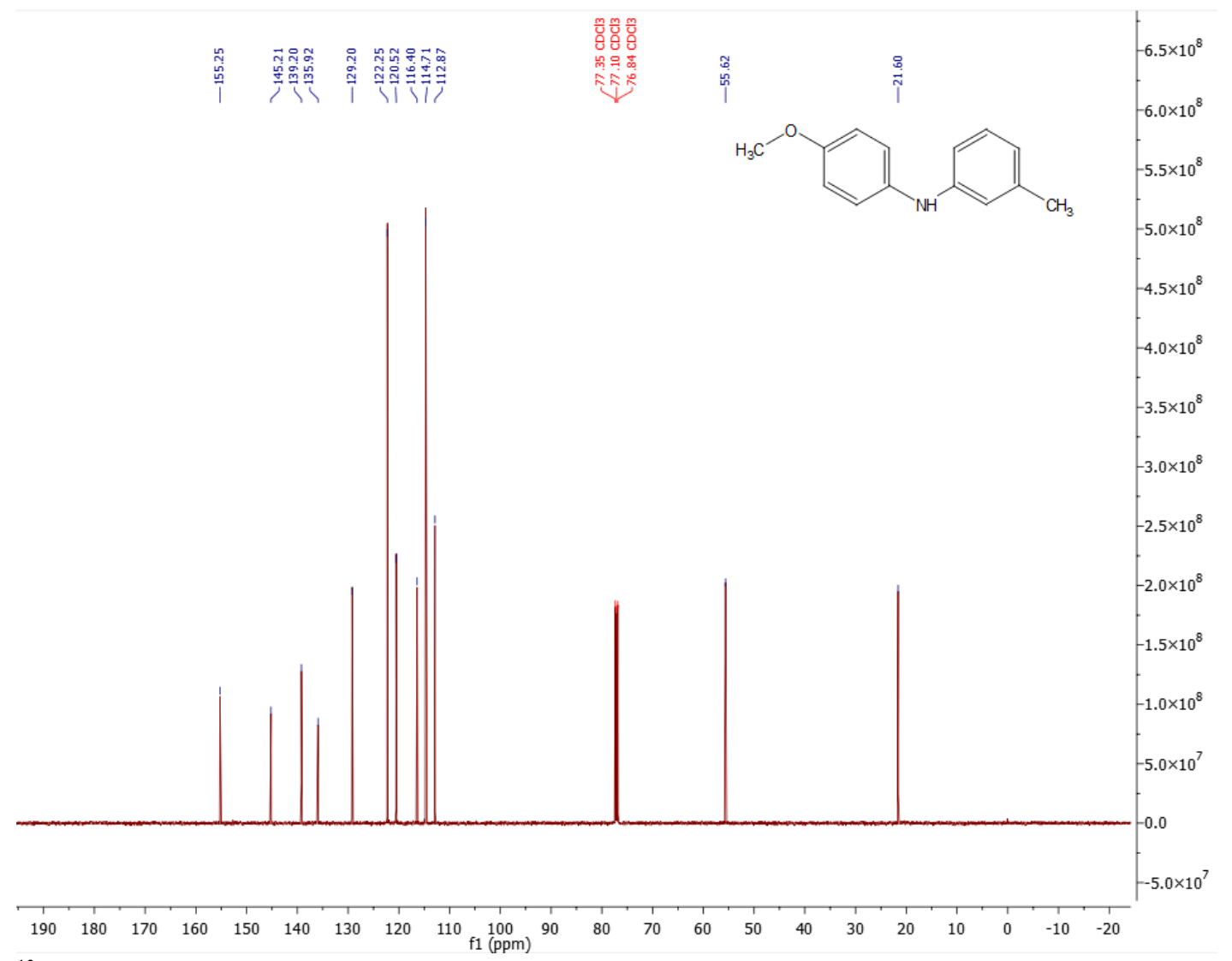

${ }^{13} \mathrm{C}$ NMR spectrum of compound $14\left(126 \mathrm{MHz}, \mathrm{CDCl}_{3}, \mathrm{rt}\right)$. 


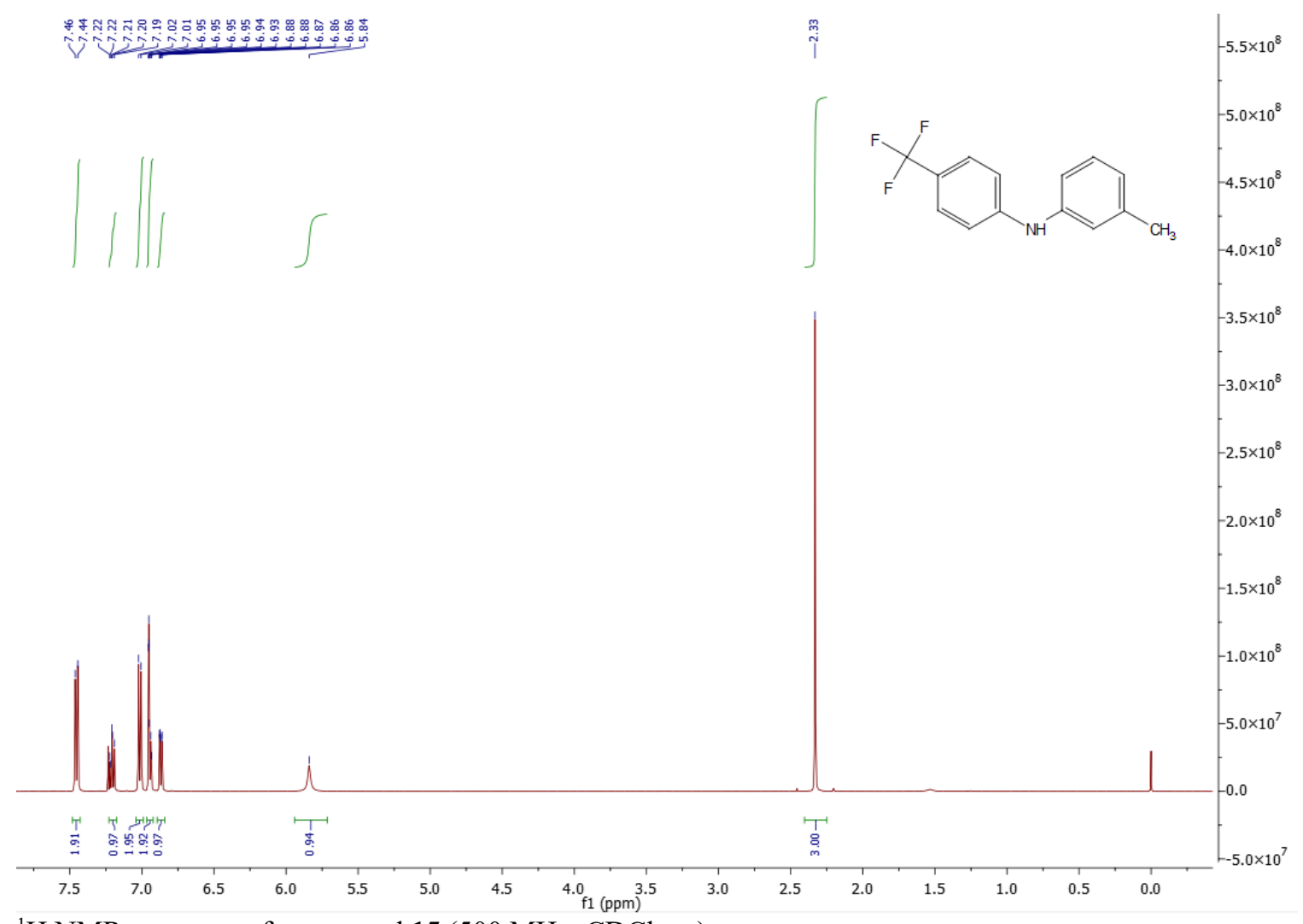

${ }^{1} \mathrm{H}$ NMR spectrum of compound $15\left(500 \mathrm{MHz}, \mathrm{CDCl}_{3}, \mathrm{rt}\right)$.

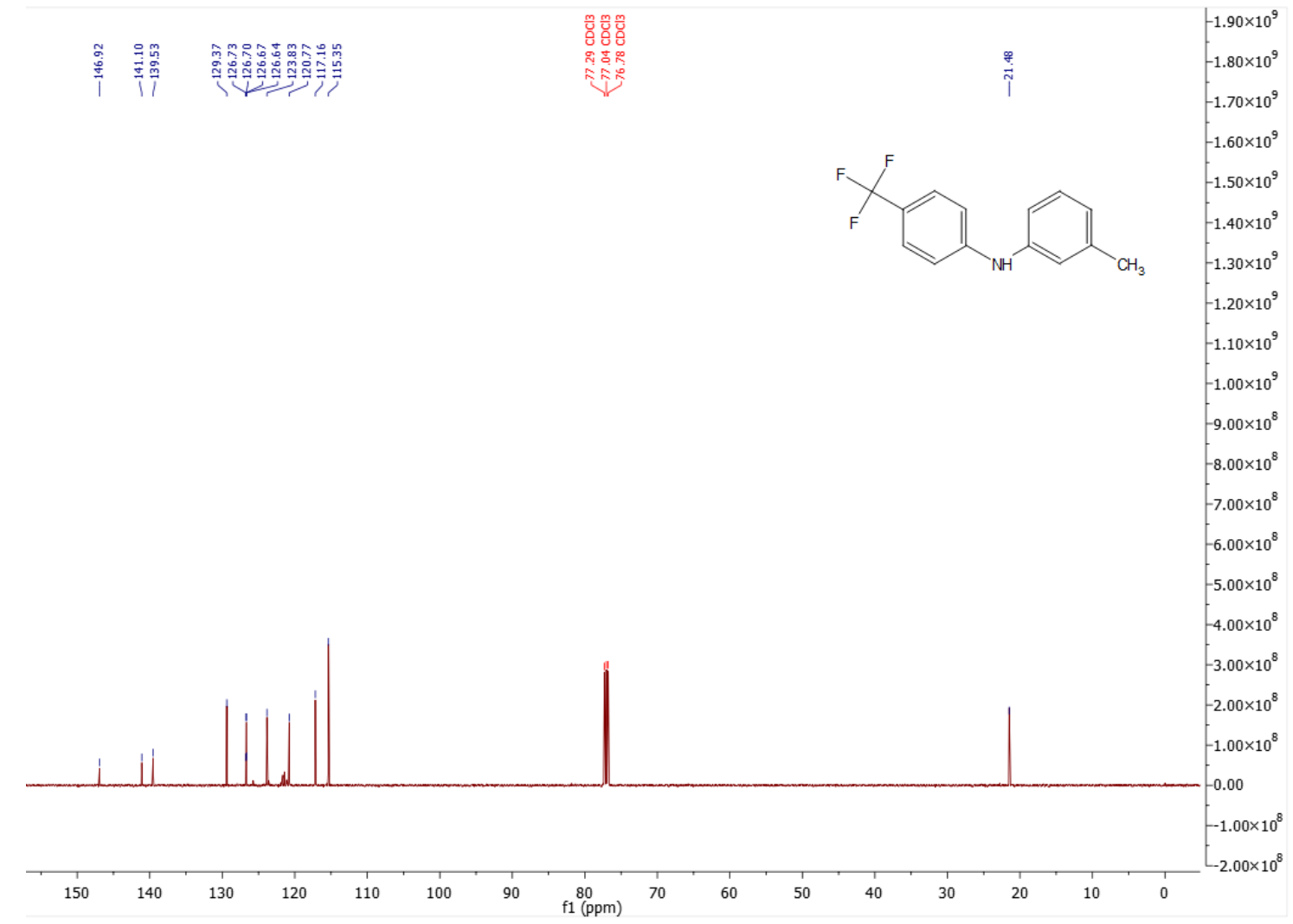

${ }^{13} \mathrm{C}$ NMR spectrum of compound $15\left(126 \mathrm{MHz}, \mathrm{CDCl}_{3}, \mathrm{rt}\right)$. 


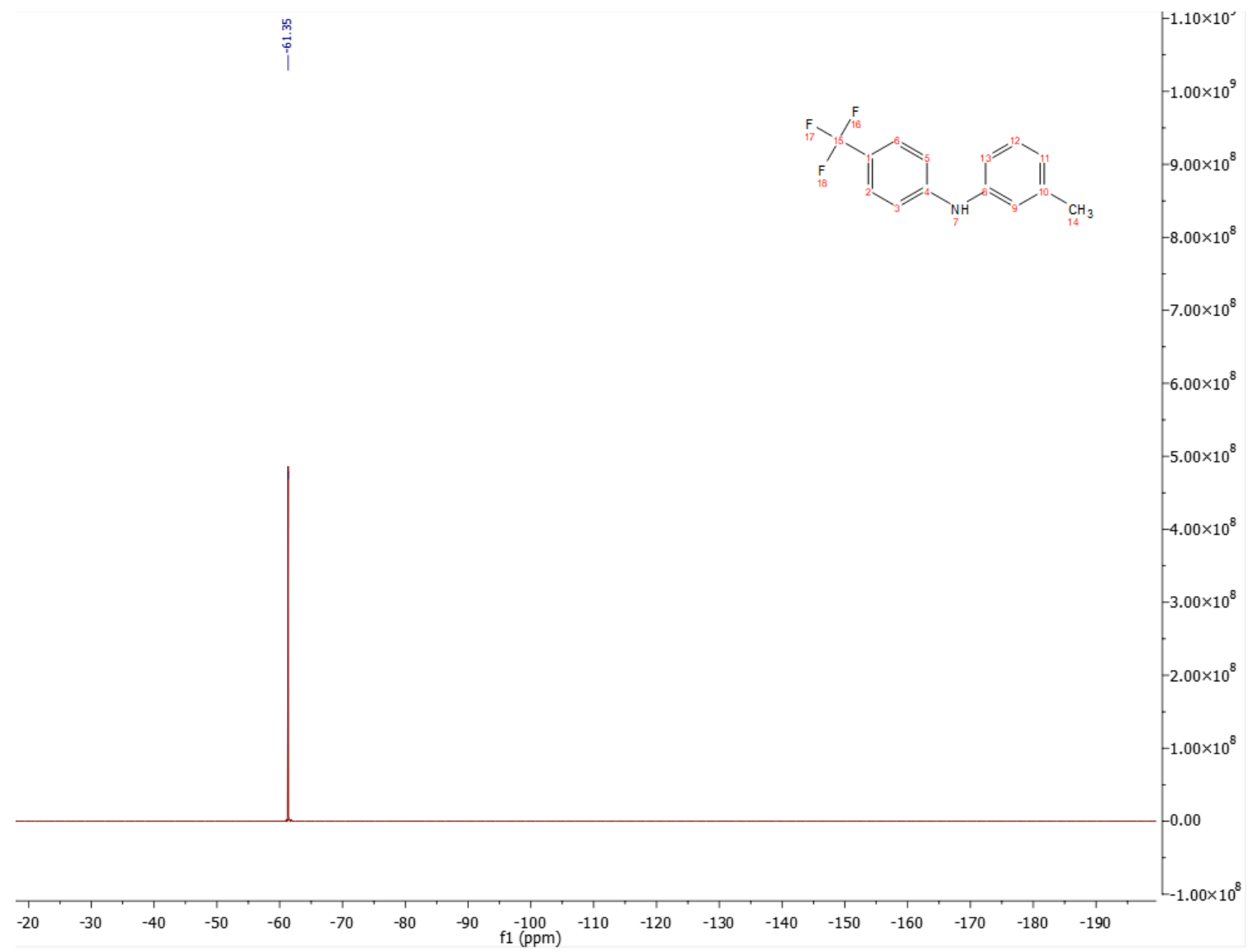

${ }^{19} \mathrm{~F}$ NMR spectrum of compound 15 (471 MHz, $\left.\mathrm{CDCl}_{3}, \mathrm{rt}\right)$. 


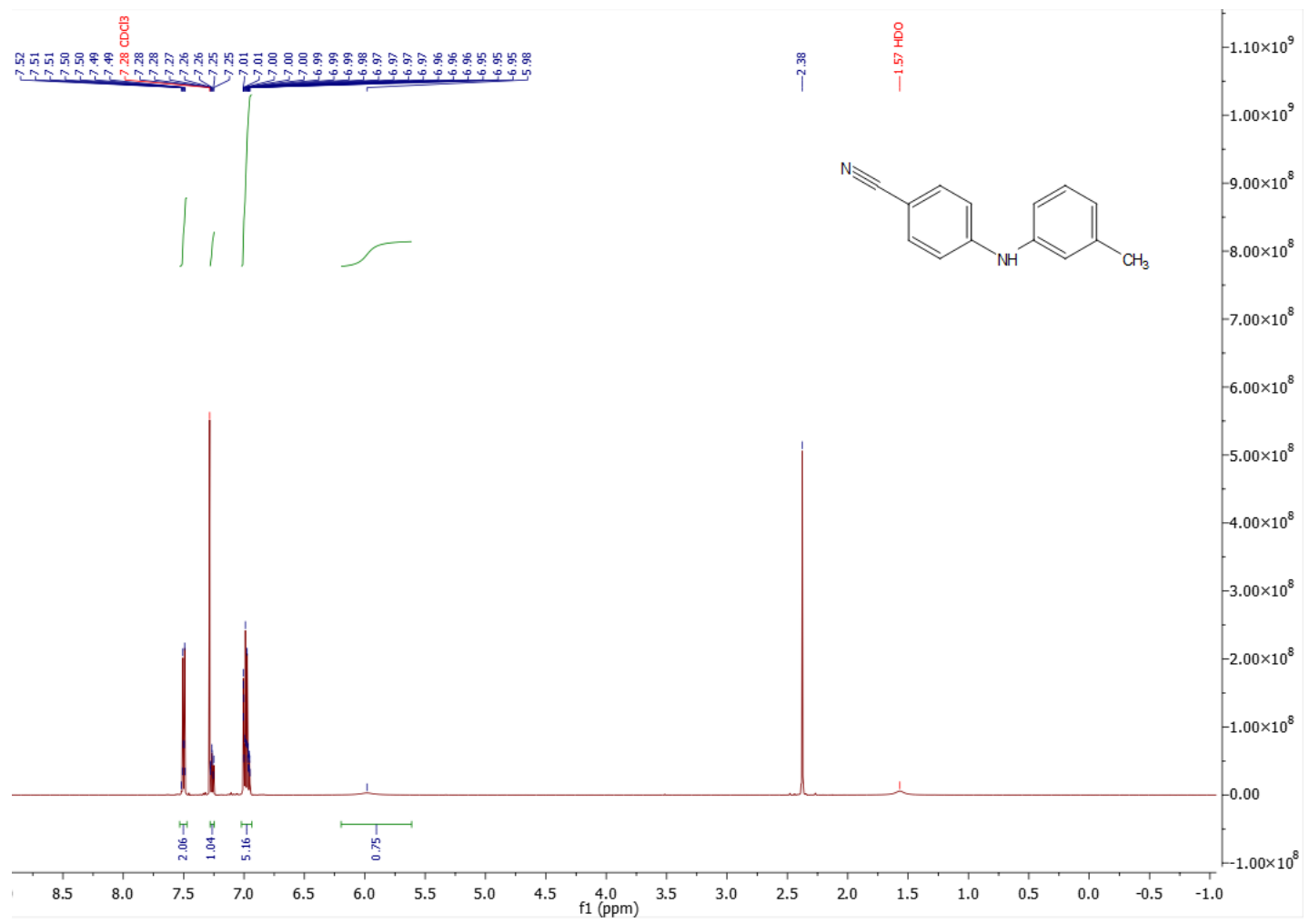

${ }^{1} \mathrm{H}$ NMR spectrum of compound $16\left(500 \mathrm{MHz}, \mathrm{CDCl}_{3}\right.$, rt).

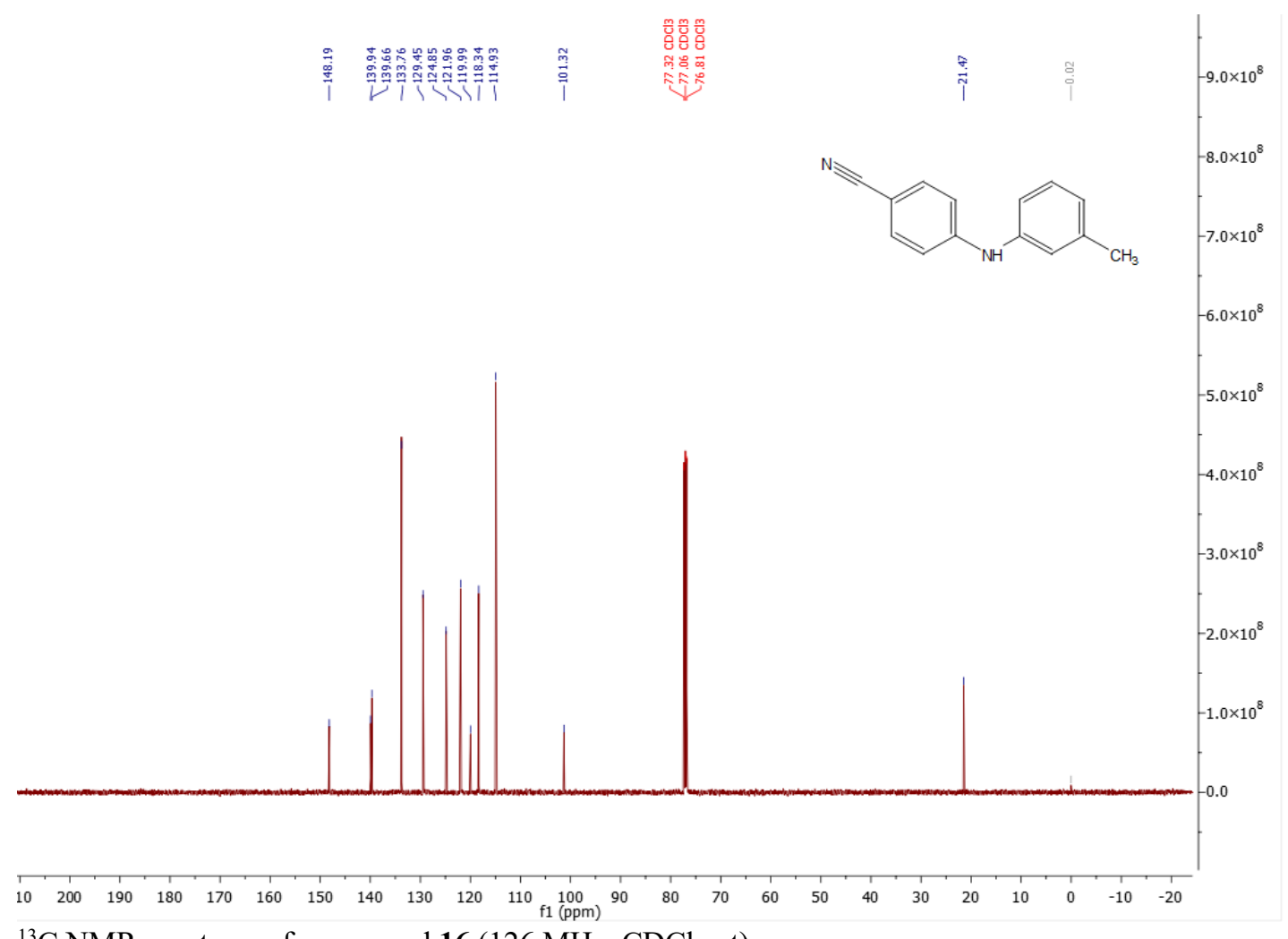

${ }^{13} \mathrm{C}$ NMR spectrum of compound $16\left(126 \mathrm{MHz}, \mathrm{CDCl}_{3}, \mathrm{rt}\right)$. 


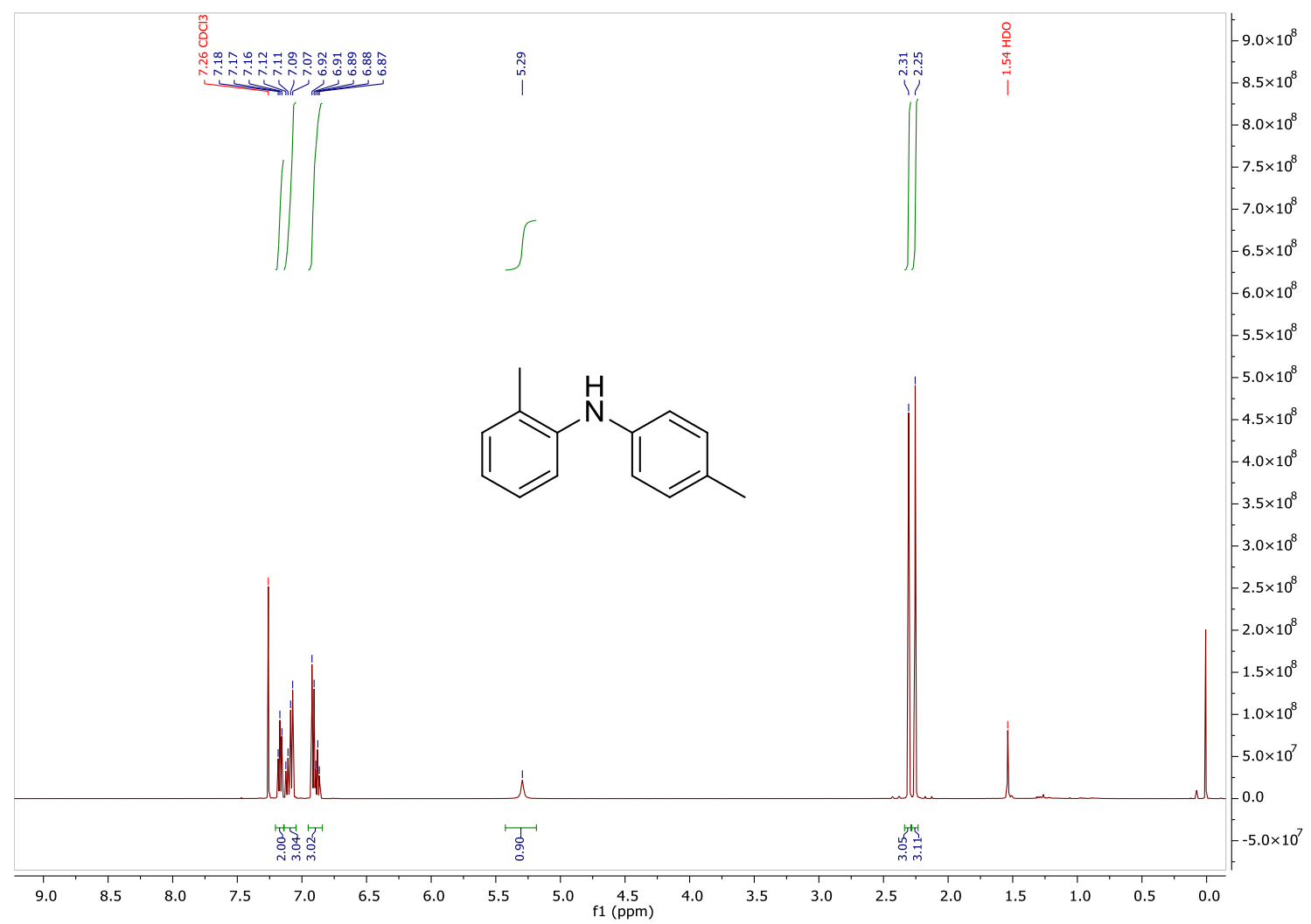

${ }^{1} \mathrm{H}$ NMR spectrum of compound $17\left(500 \mathrm{MHz}, \mathrm{CDCl}_{3}, \mathrm{rt}\right)$.

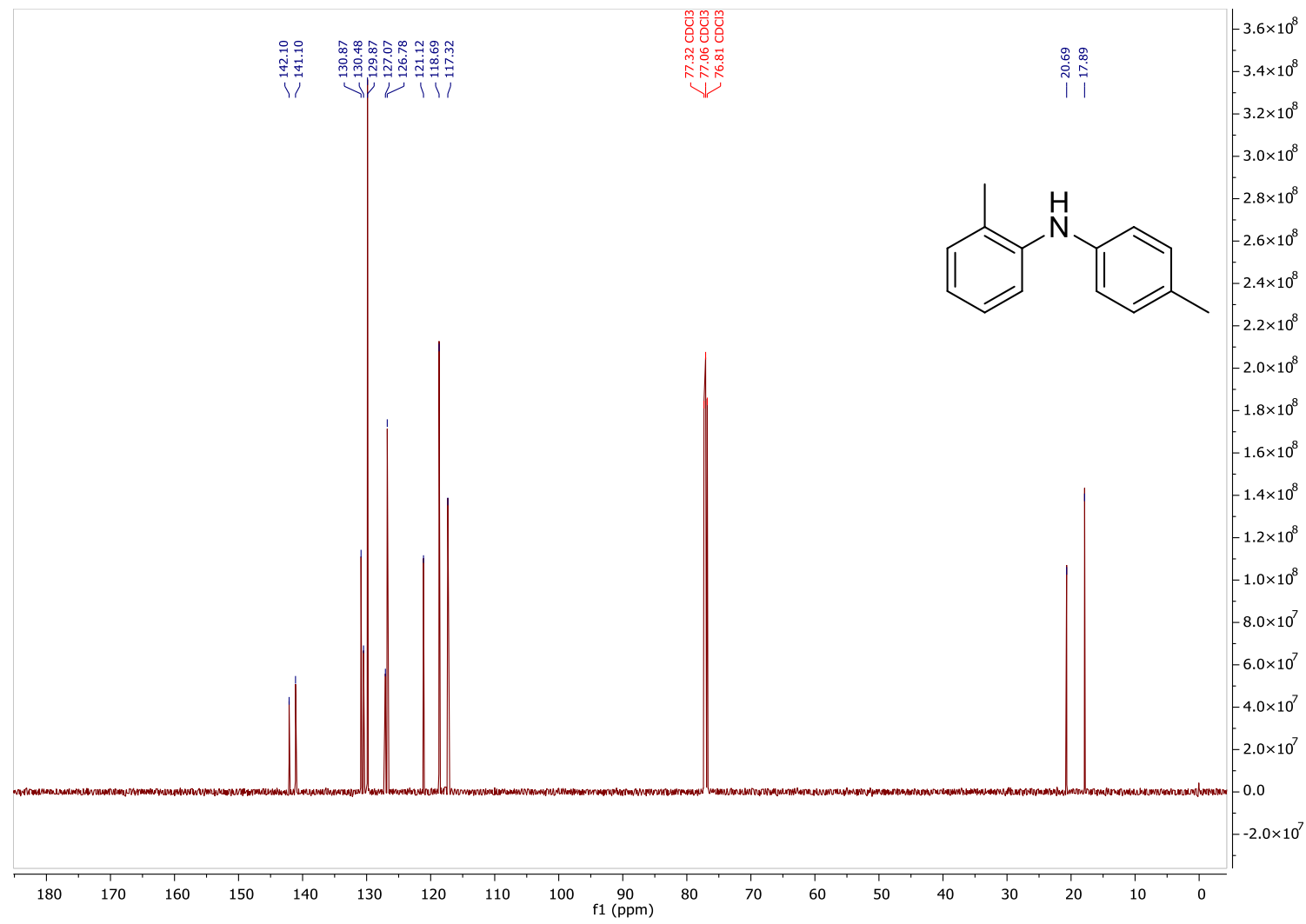

${ }^{13} \mathrm{C}$ NMR spectrum of compound $17\left(126 \mathrm{MHz}, \mathrm{CDCl}_{3}, \mathrm{rt}\right)$. 


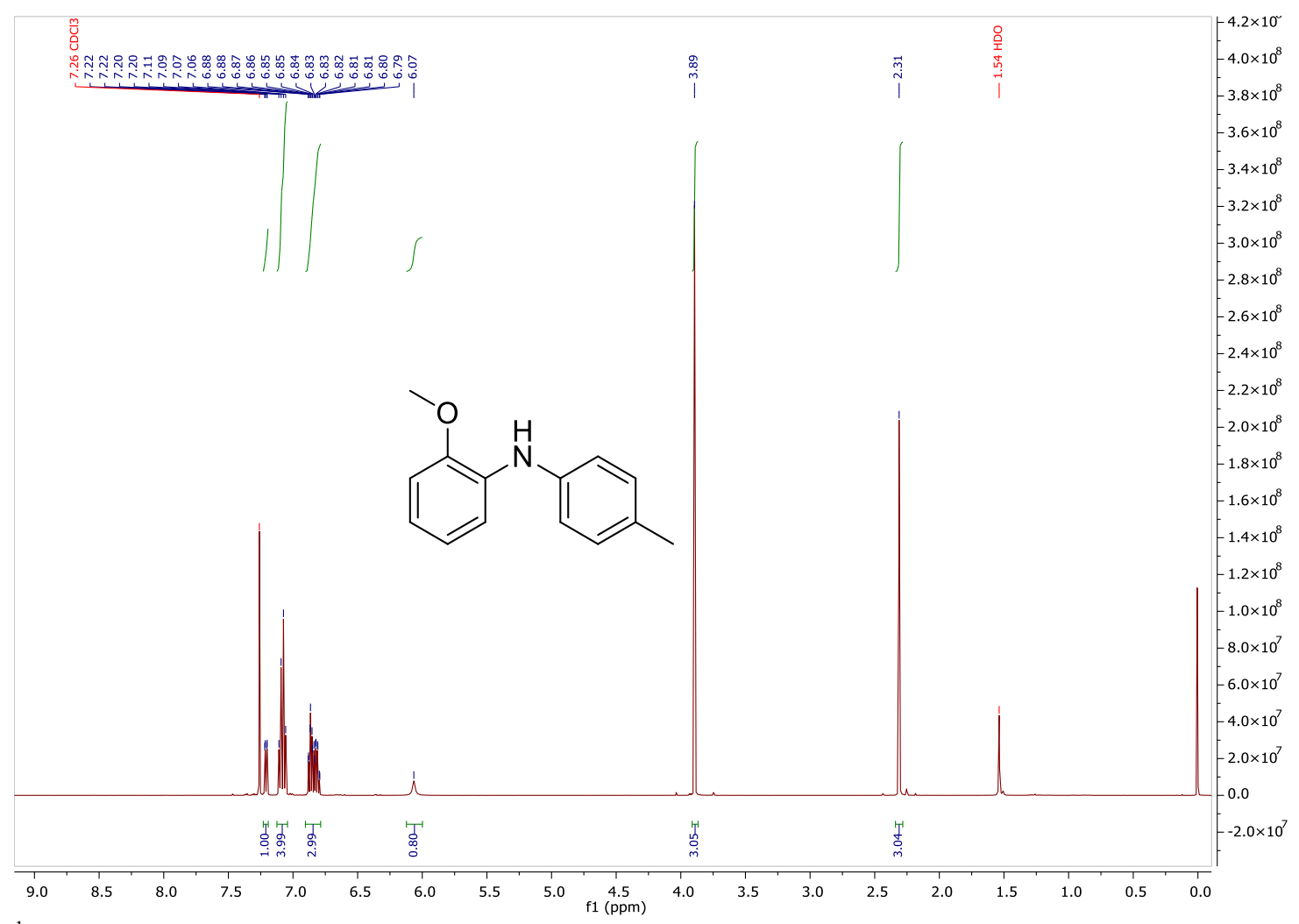

${ }^{1} \mathrm{H}$ NMR spectrum of compound $18\left(500 \mathrm{MHz}, \mathrm{CDCl}_{3}, \mathrm{rt}\right)$.

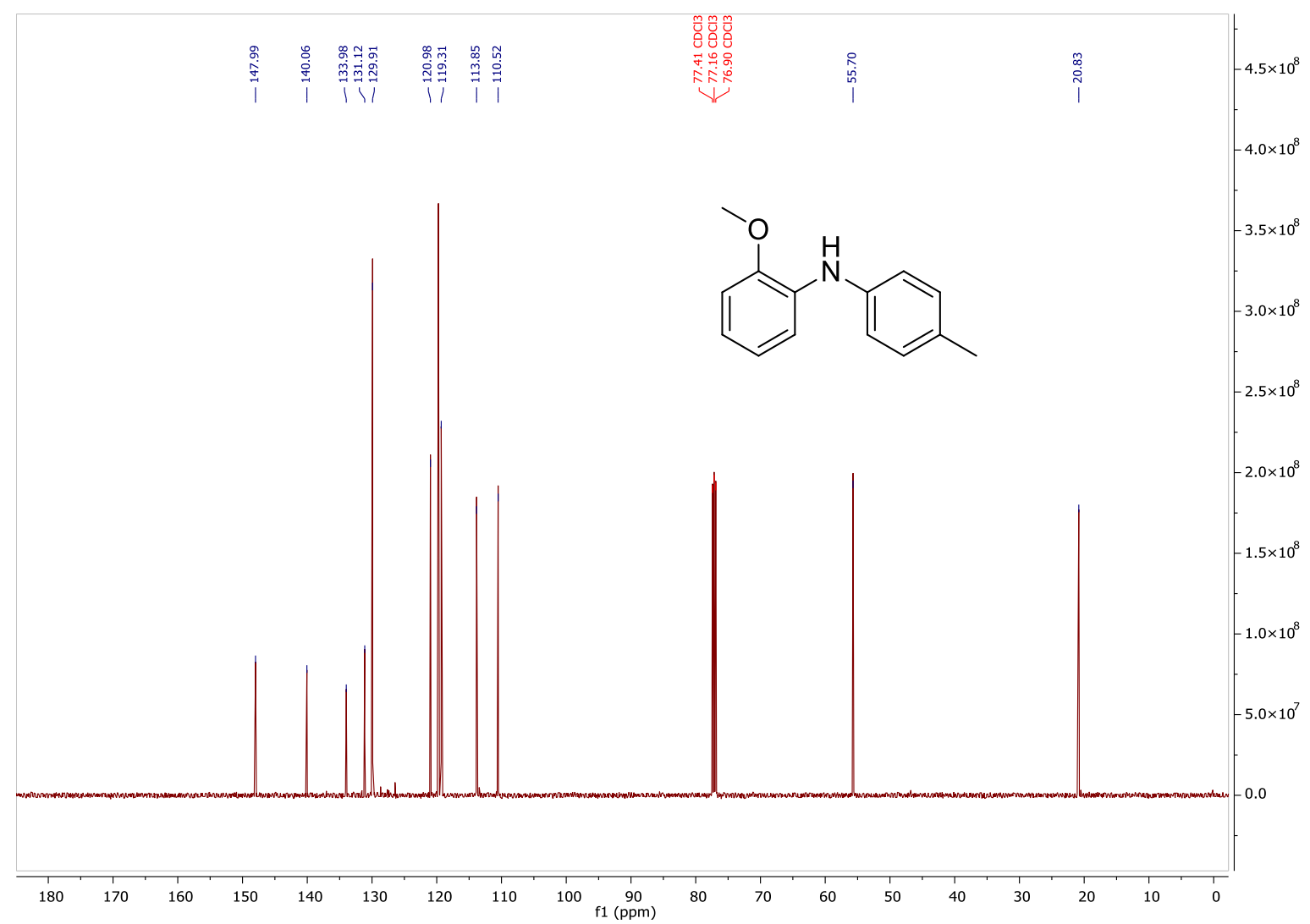

${ }^{13} \mathrm{C}$ NMR spectrum of compound $18\left(126 \mathrm{MHz}, \mathrm{CDCl}_{3}, \mathrm{rt}\right)$. 


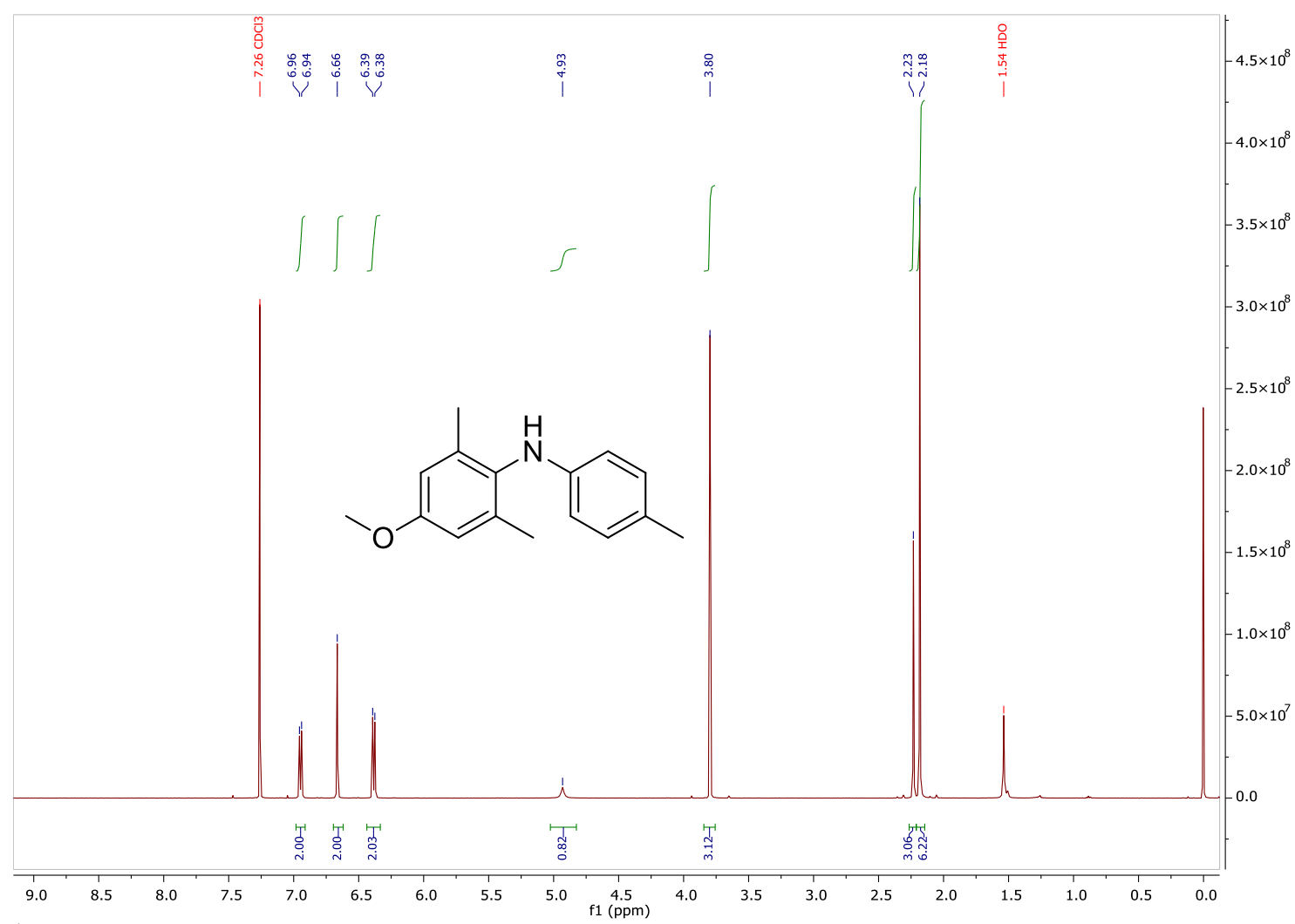

${ }^{1} \mathrm{H}$ NMR spectrum of compound $19\left(500 \mathrm{MHz}, \mathrm{CDCl}_{3}, \mathrm{rt}\right.$ ).

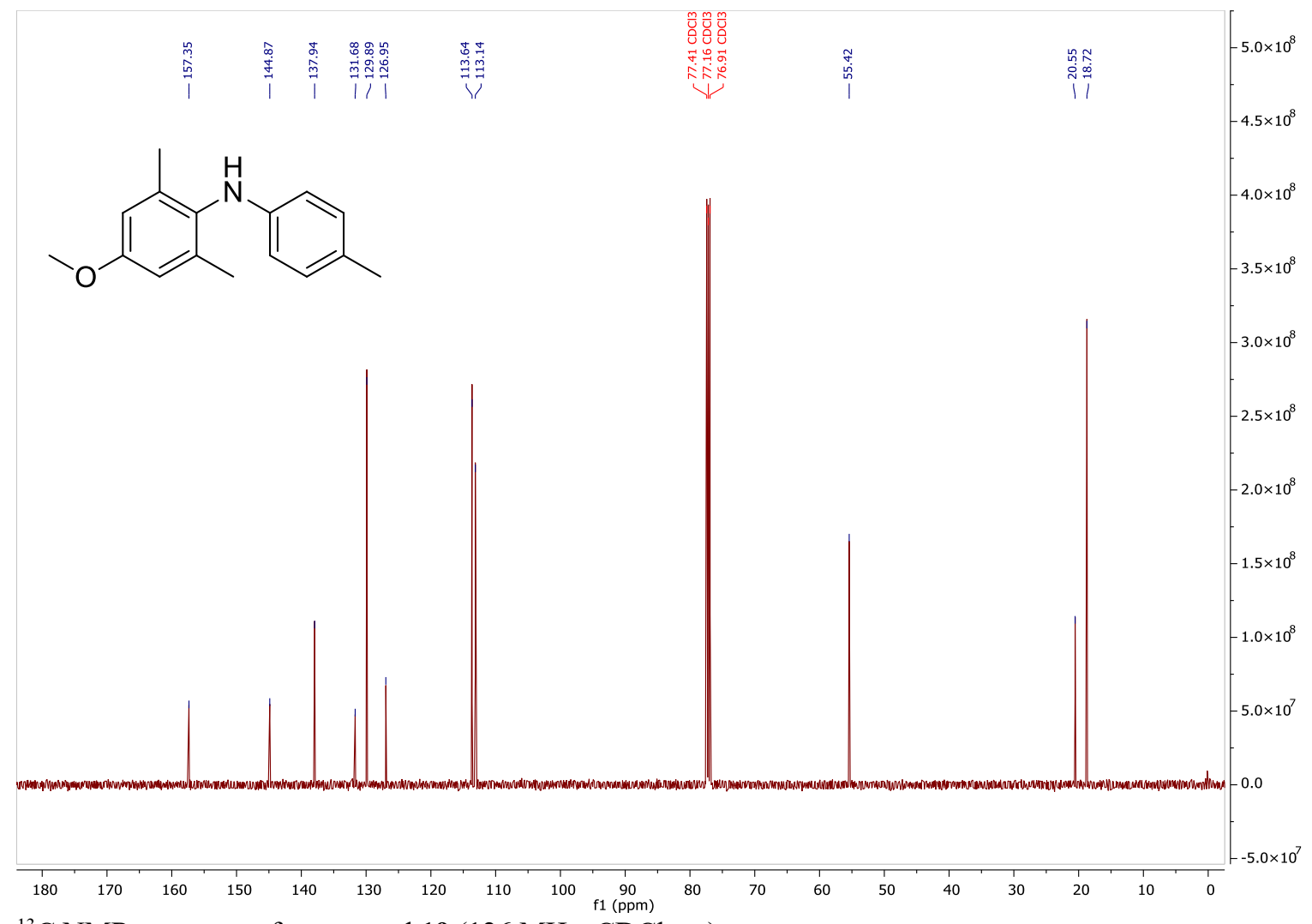

${ }^{13} \mathrm{C}$ NMR spectrum of compound $19\left(126 \mathrm{MHz}, \mathrm{CDCl}_{3}, \mathrm{rt}\right)$. 


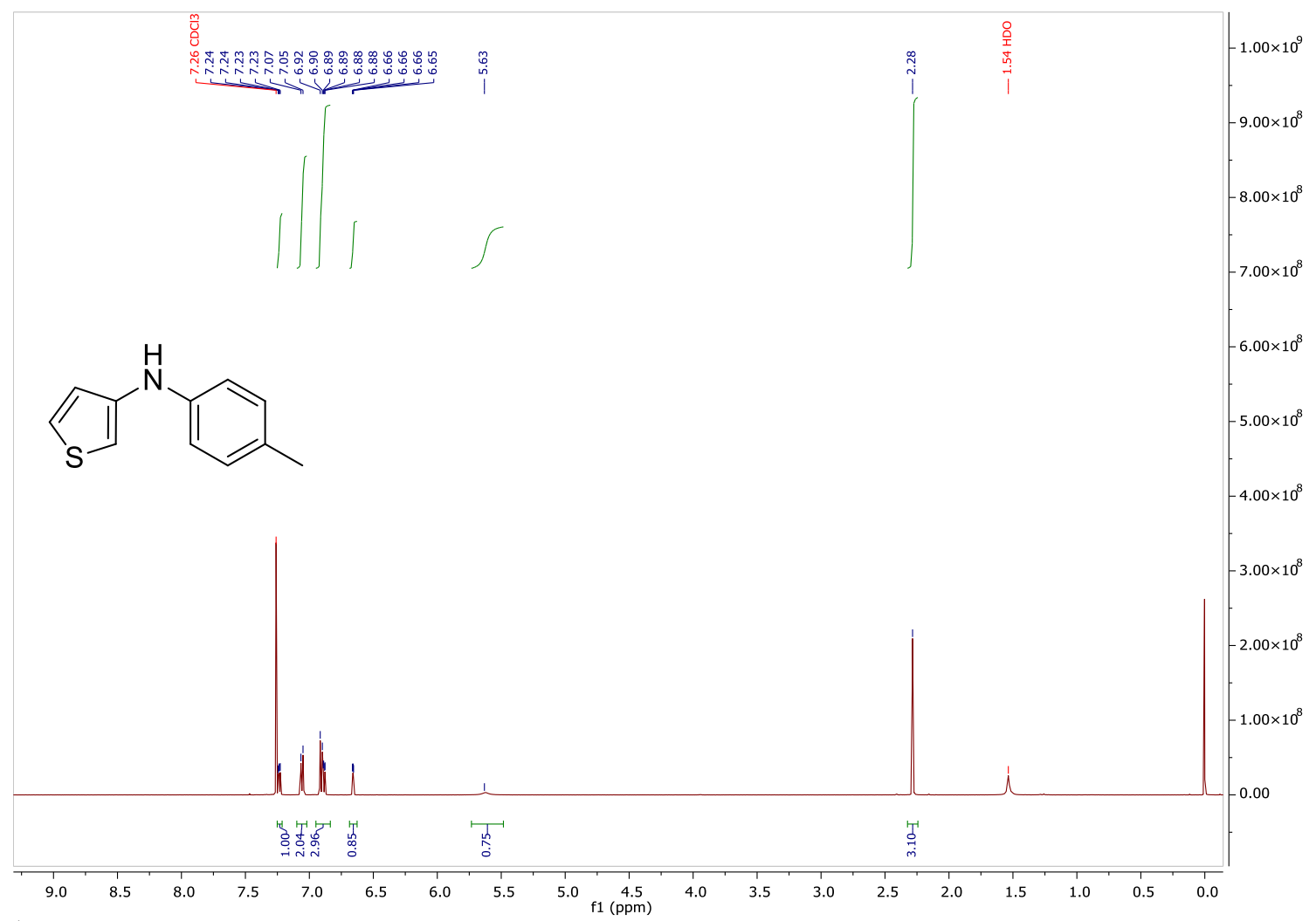

${ }^{1} \mathrm{H}$ NMR spectrum of compound $20\left(500 \mathrm{MHz}, \mathrm{CDCl}_{3}, \mathrm{rt}\right)$.

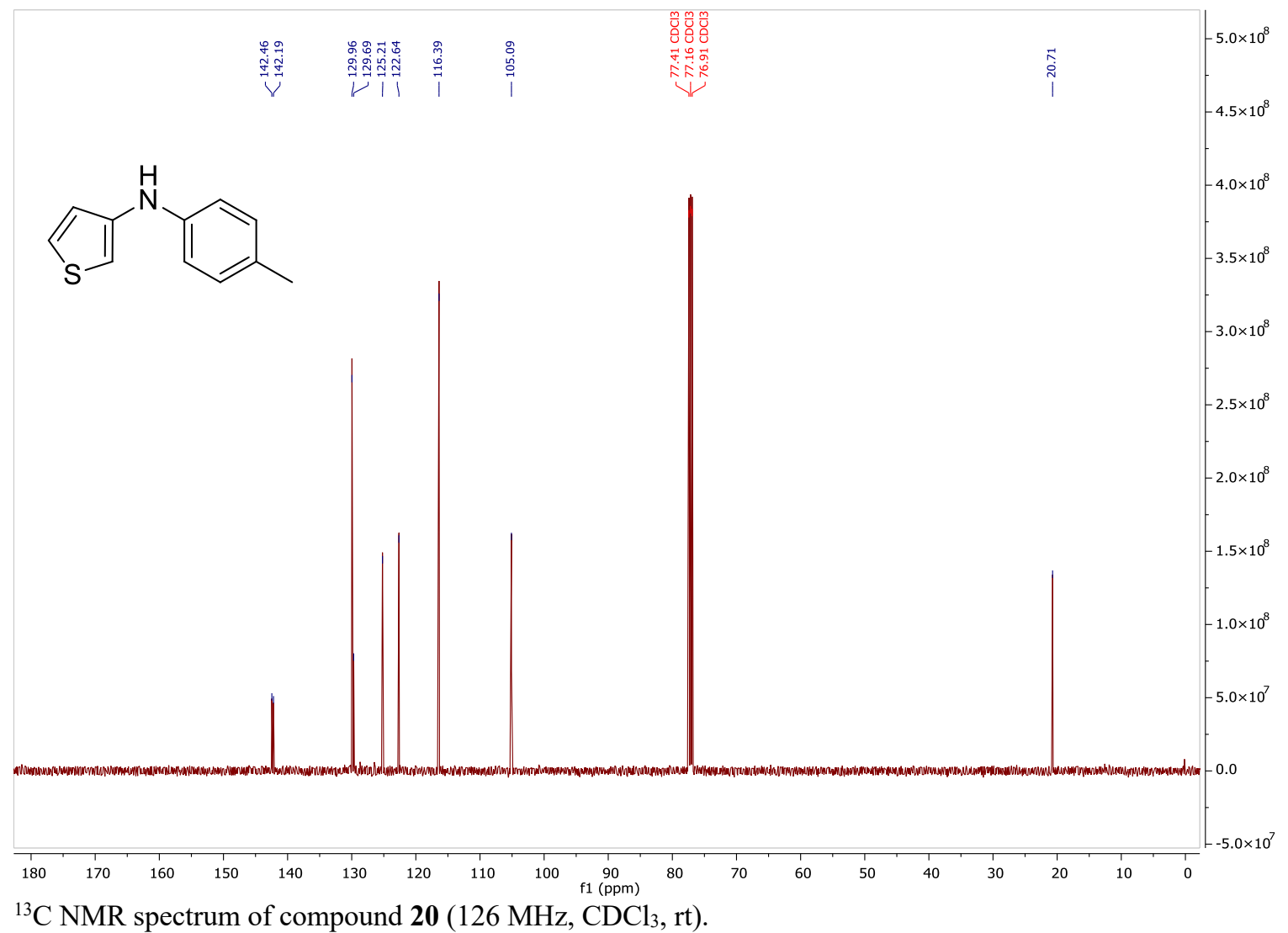




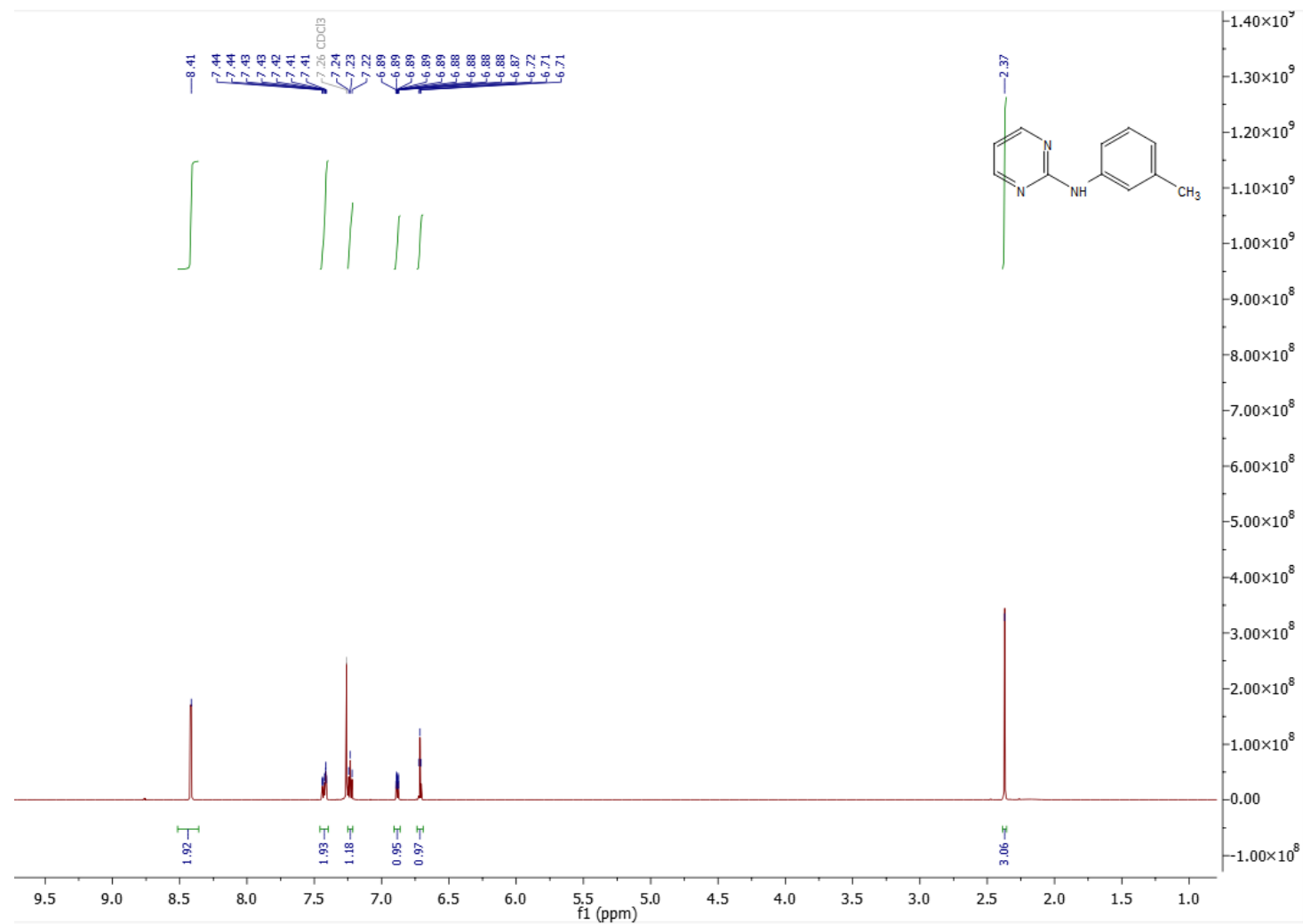

${ }^{1} \mathrm{H}$ NMR spectrum of compound $21\left(500 \mathrm{MHz}, \mathrm{CDCl}_{3}, \mathrm{rt}\right)$.

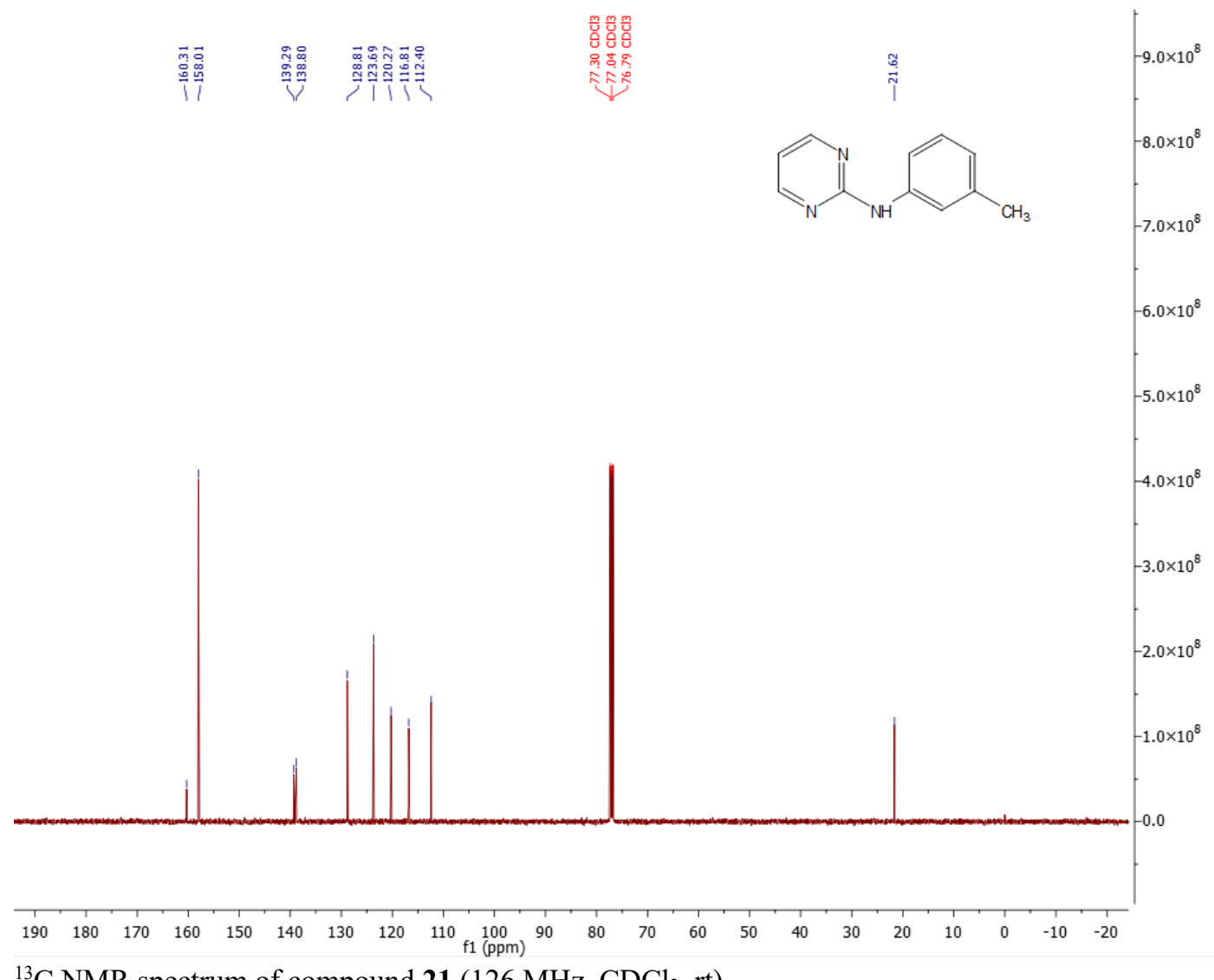

${ }^{13} \mathrm{C}$ NMR spectrum of compound $21\left(126 \mathrm{MHz}, \mathrm{CDCl}_{3}, \mathrm{rt}\right)$. 


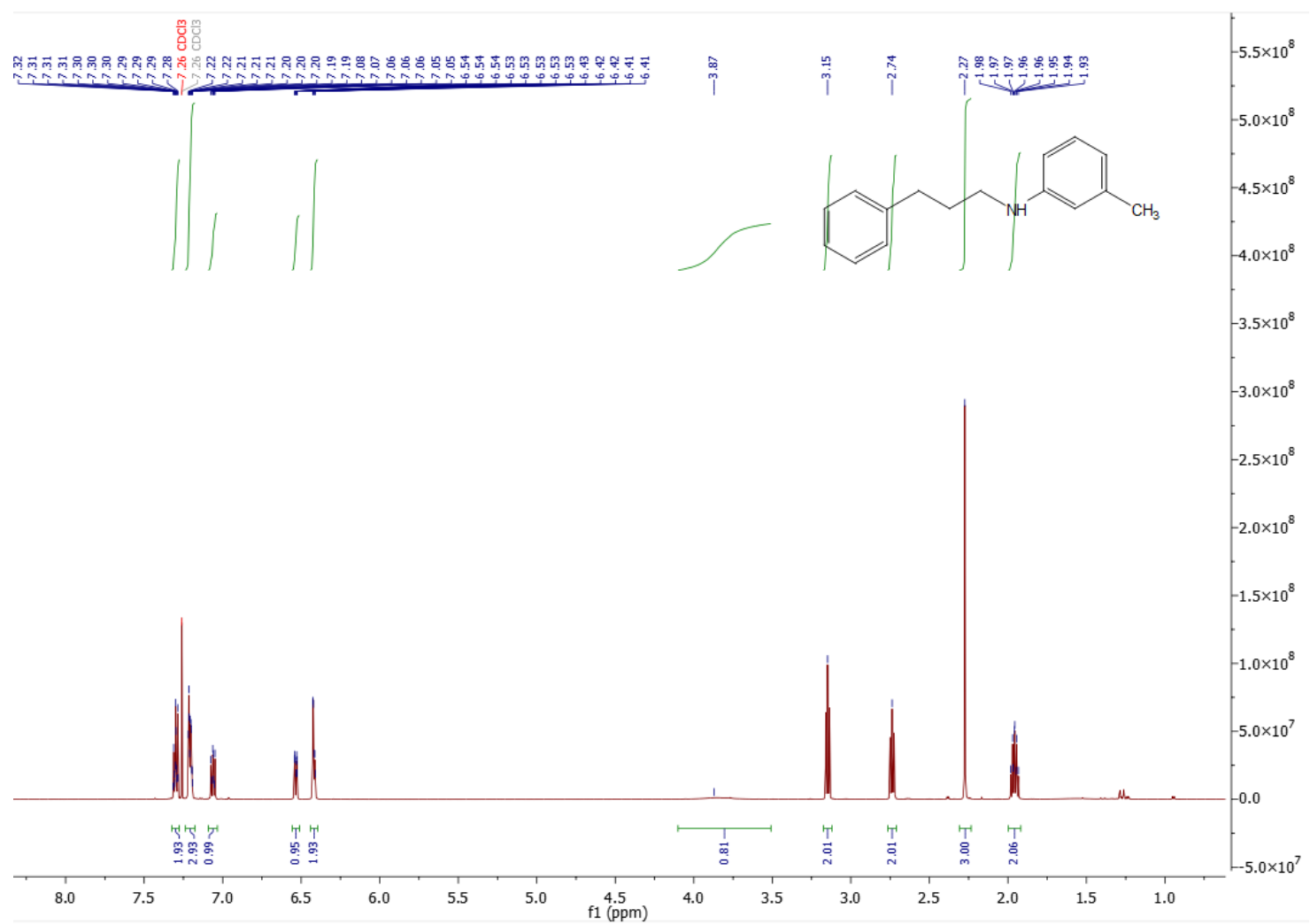

${ }^{1} \mathrm{H}$ NMR spectrum of compound $22\left(500 \mathrm{MHz}, \mathrm{CDCl}_{3}, \mathrm{rt}\right)$.

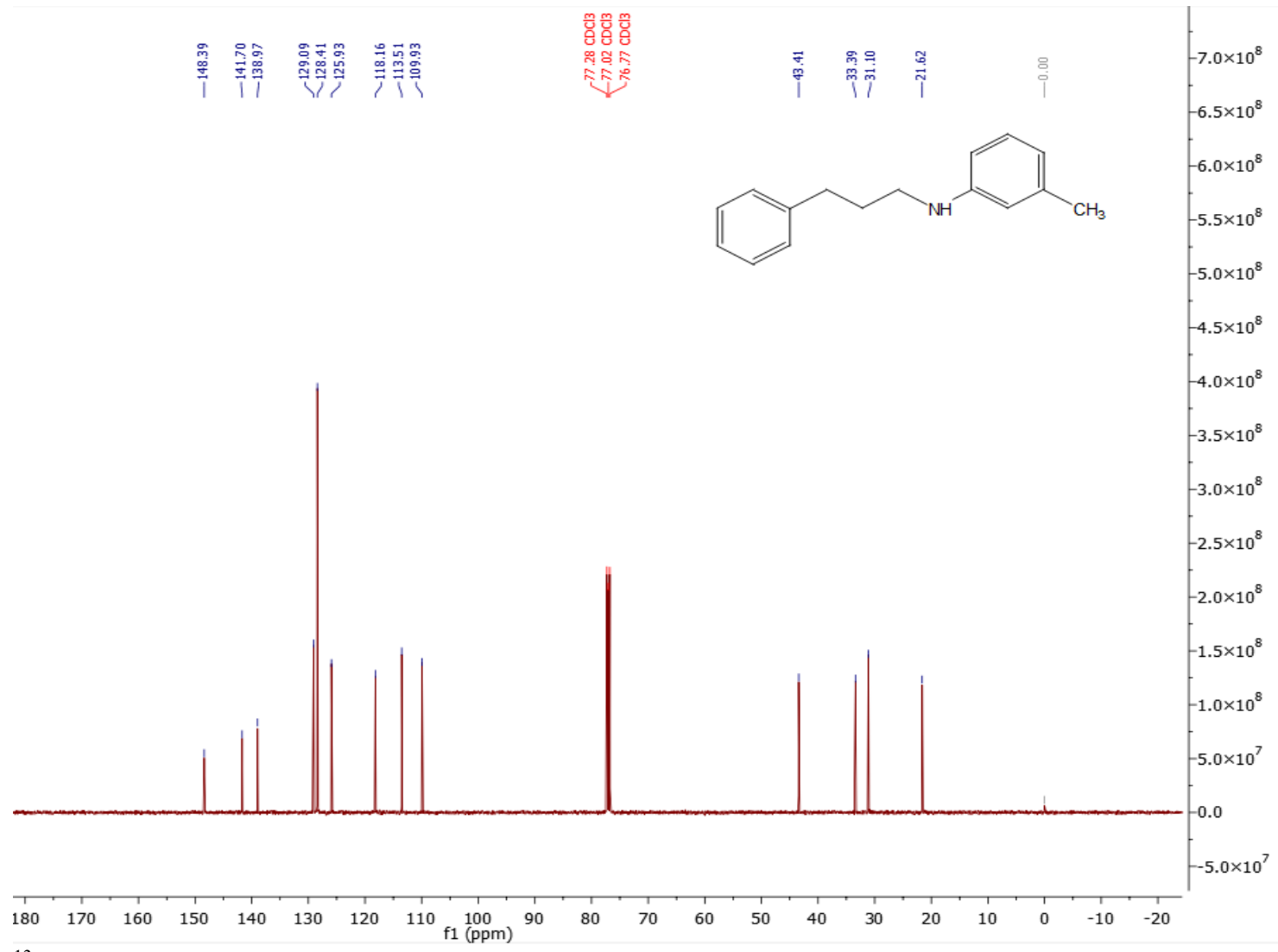

${ }^{13} \mathrm{C}$ NMR spectrum of compound $22\left(126 \mathrm{MHz}, \mathrm{CDCl}_{3}, \mathrm{rt}\right)$. 


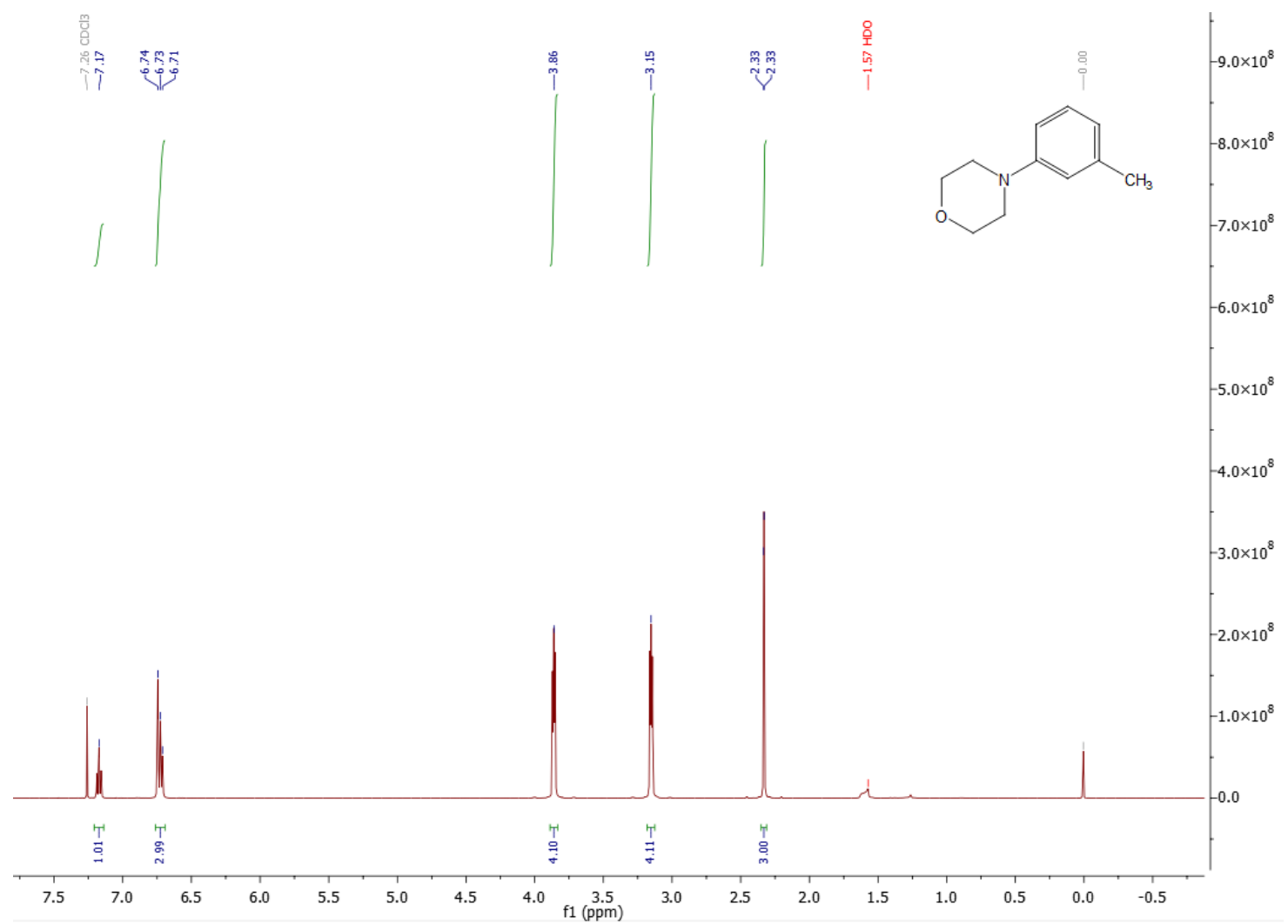

${ }^{1} \mathrm{H}$ NMR spectrum of compound $23\left(500 \mathrm{MHz}, \mathrm{CDCl}_{3}, \mathrm{rt}\right)$.

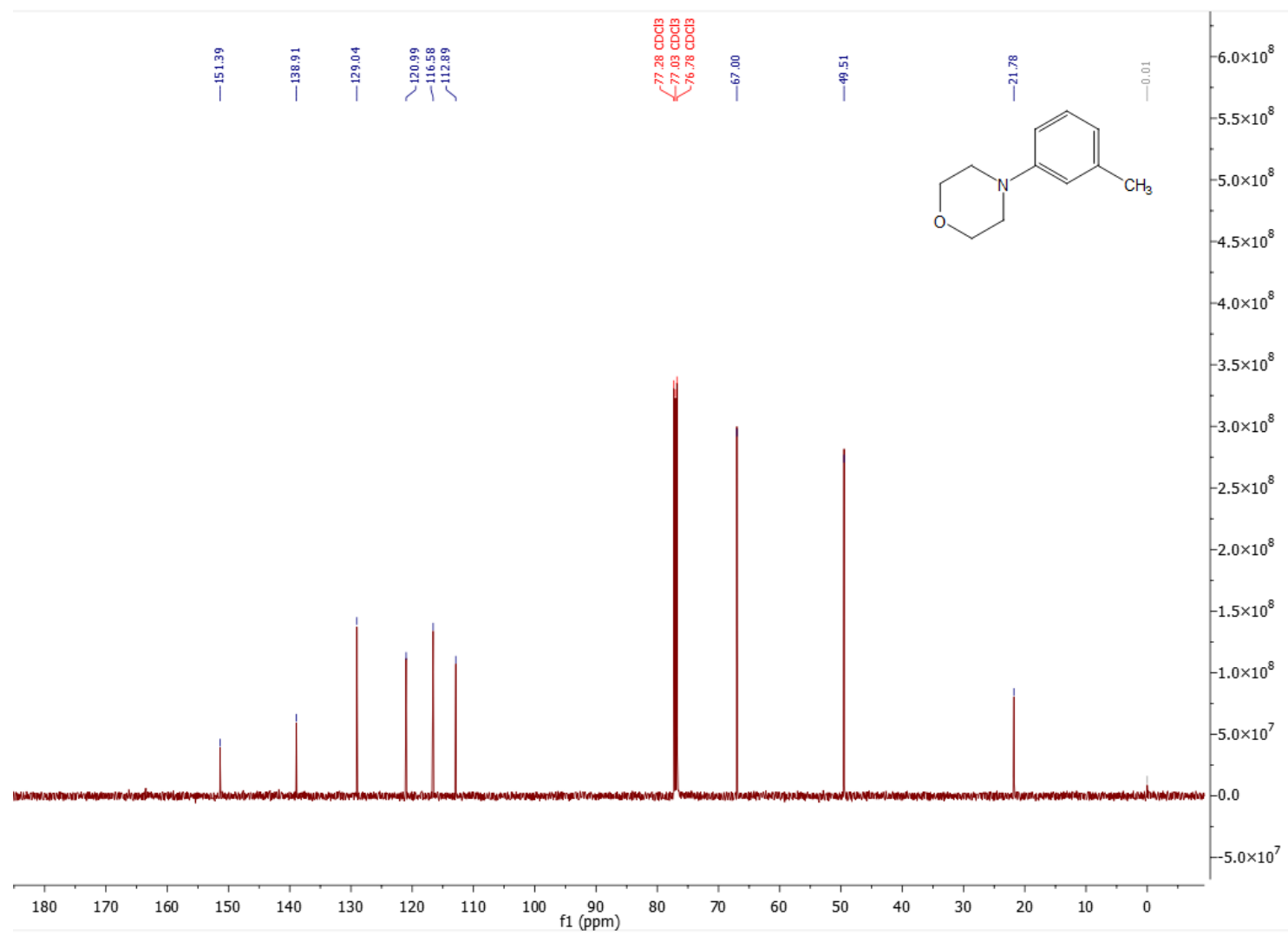

${ }^{13} \mathrm{C}$ NMR spectrum of compound $23\left(126 \mathrm{MHz}, \mathrm{CDCl}_{3}\right.$, rt). 


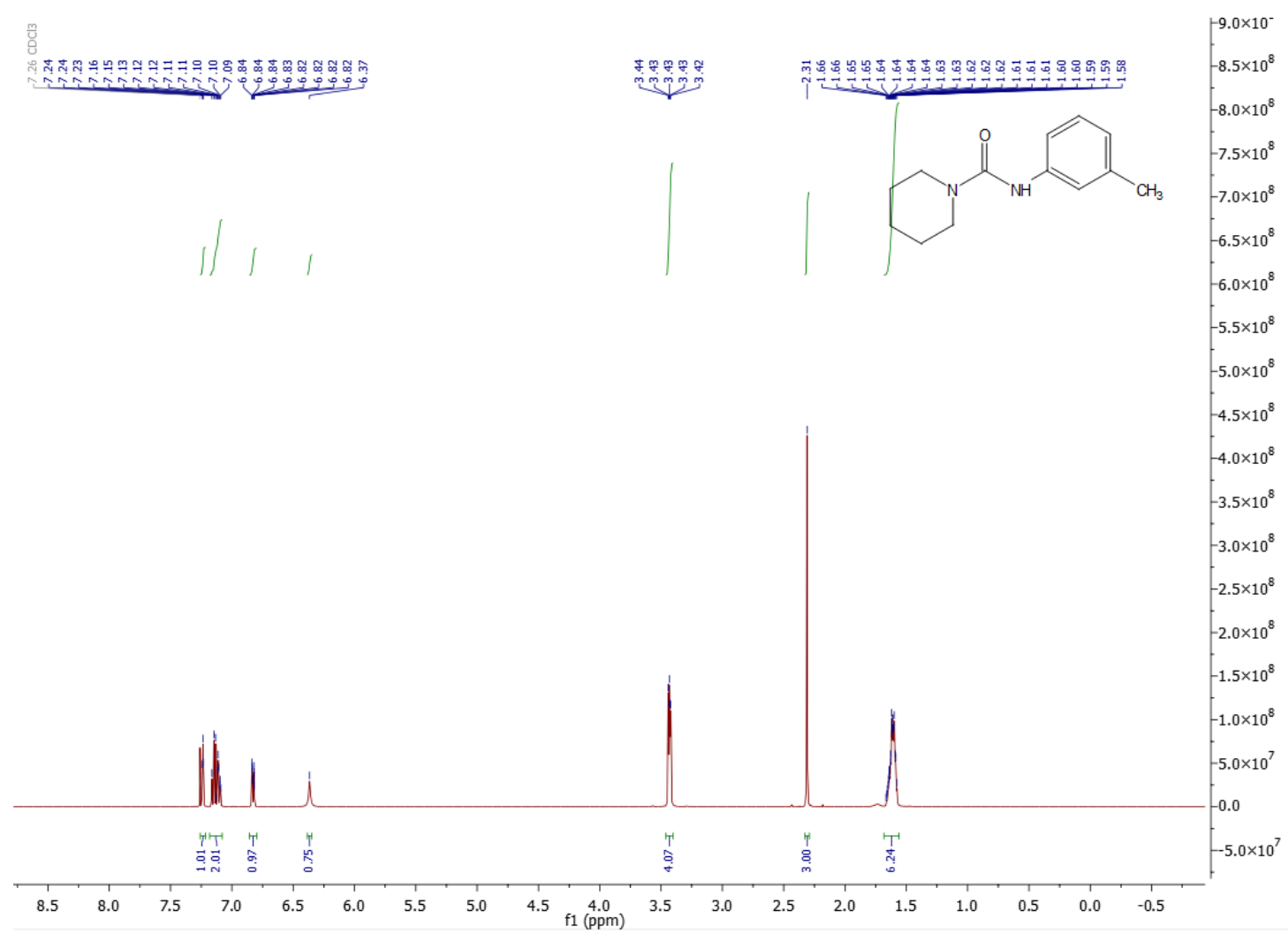

${ }^{1} \mathrm{H}$ NMR spectrum of compound $24\left(500 \mathrm{MHz}, \mathrm{CDCl}_{3}, \mathrm{rt}\right)$.

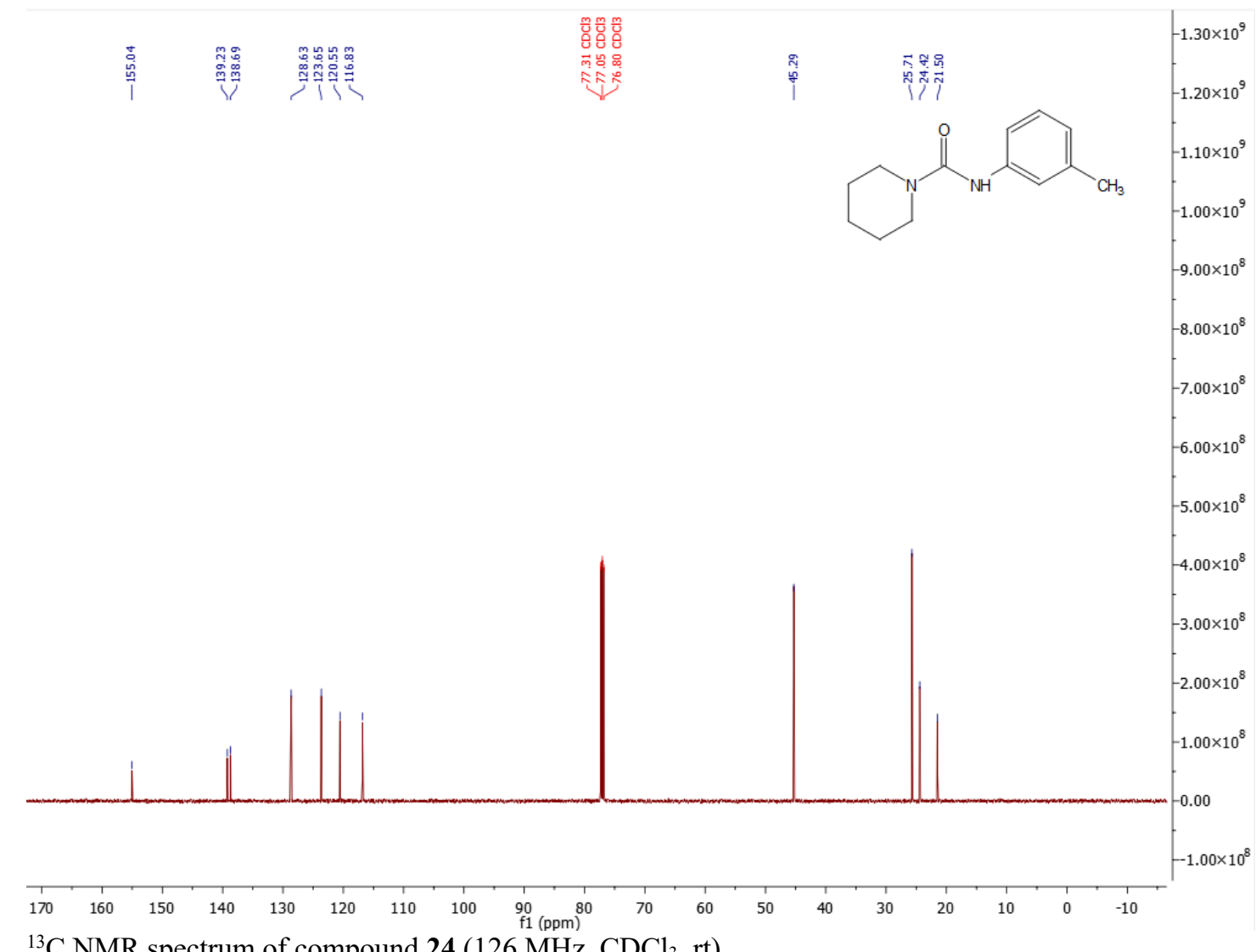

${ }^{13} \mathrm{C}$ NMR spectrum of compound $24\left(126 \mathrm{MHz}, \mathrm{CDCl}_{3}, \mathrm{rt}\right)$. 


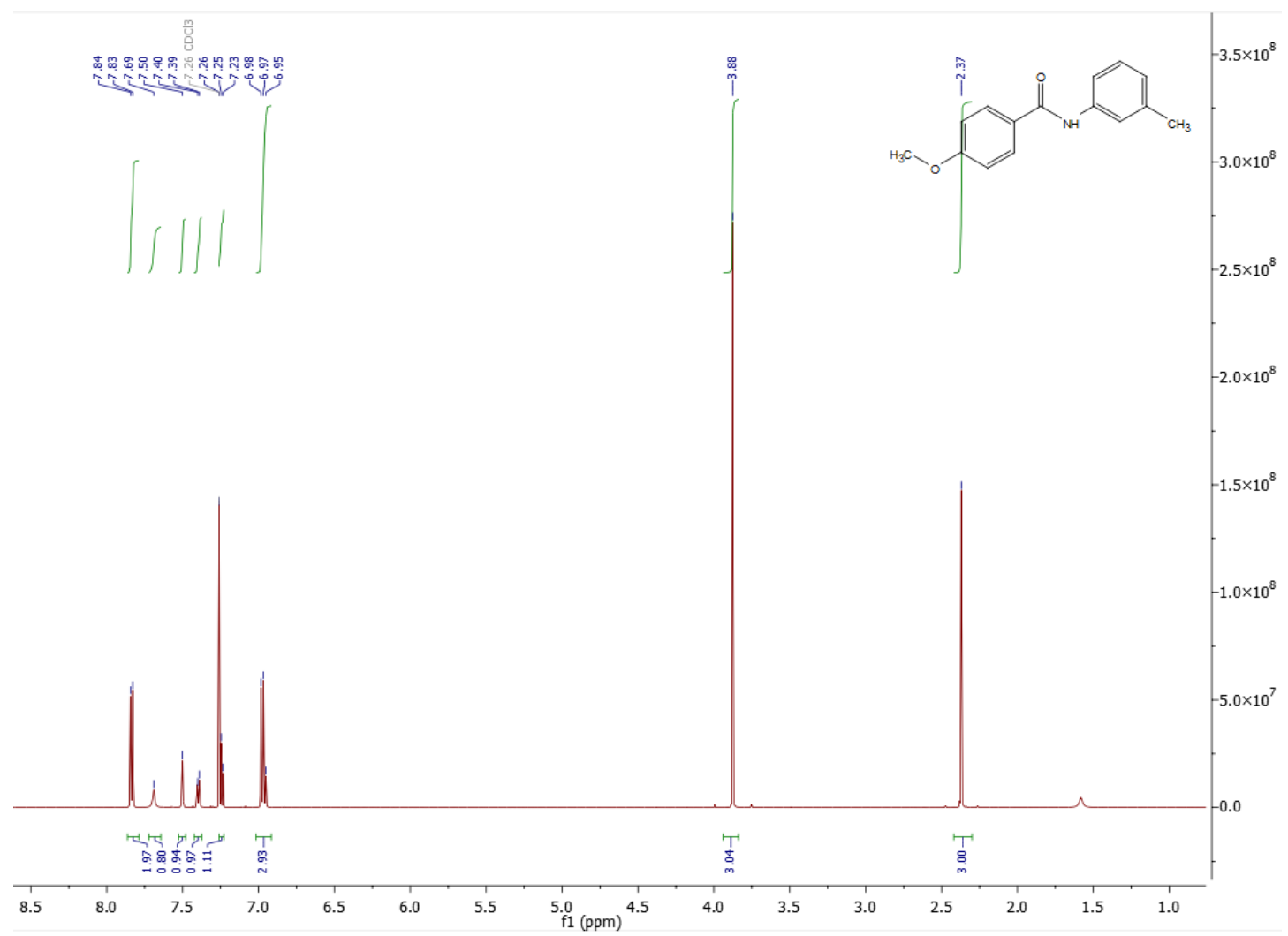

${ }^{1} \mathrm{H}$ NMR spectrum of compound $25\left(500 \mathrm{MHz}, \mathrm{CDCl}_{3}\right.$, rt).

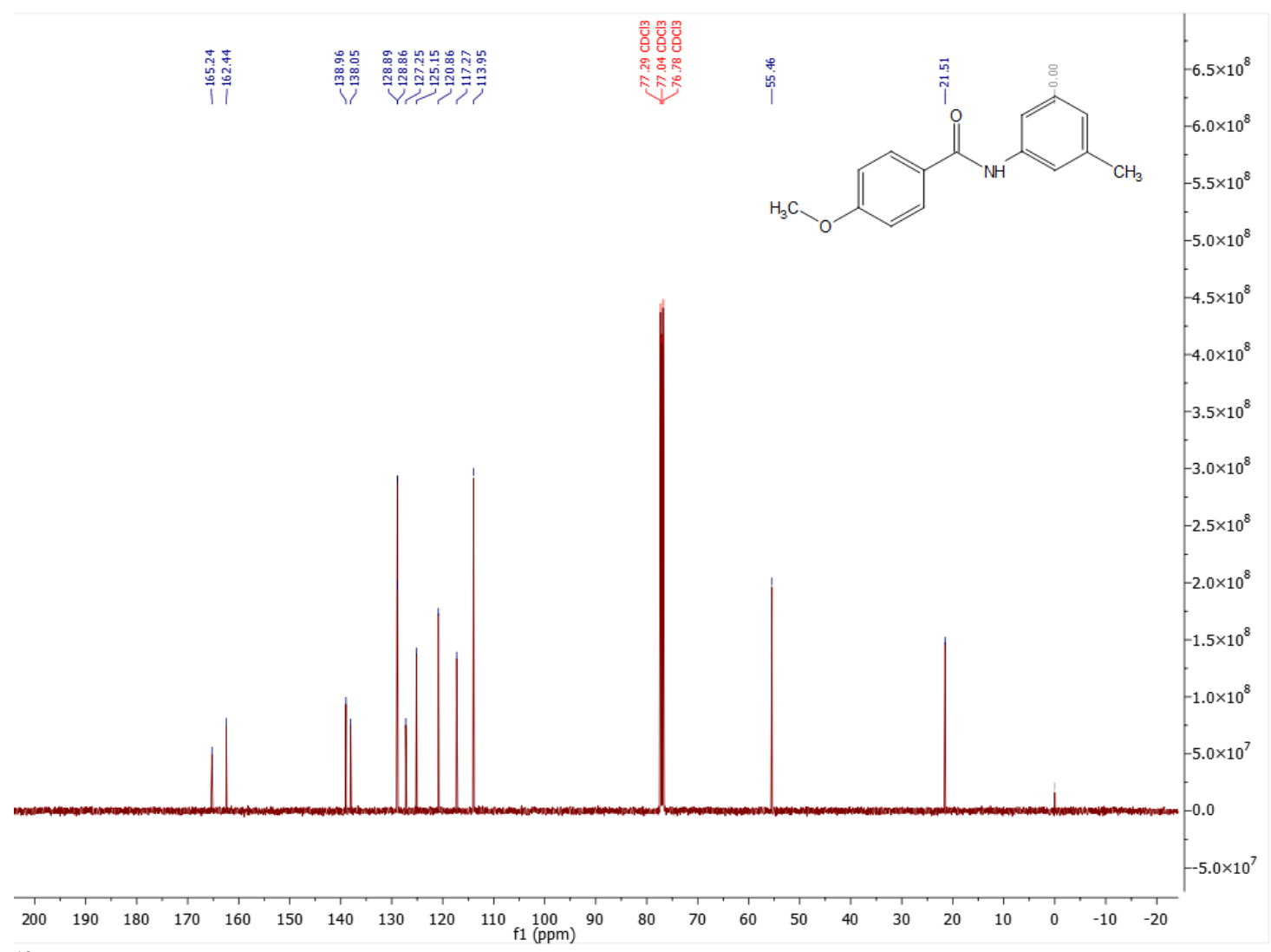

${ }^{13} \mathrm{C}$ NMR spectrum of compound $25\left(126 \mathrm{MHz}, \mathrm{CDCl}_{3}, \mathrm{rt}\right)$. 


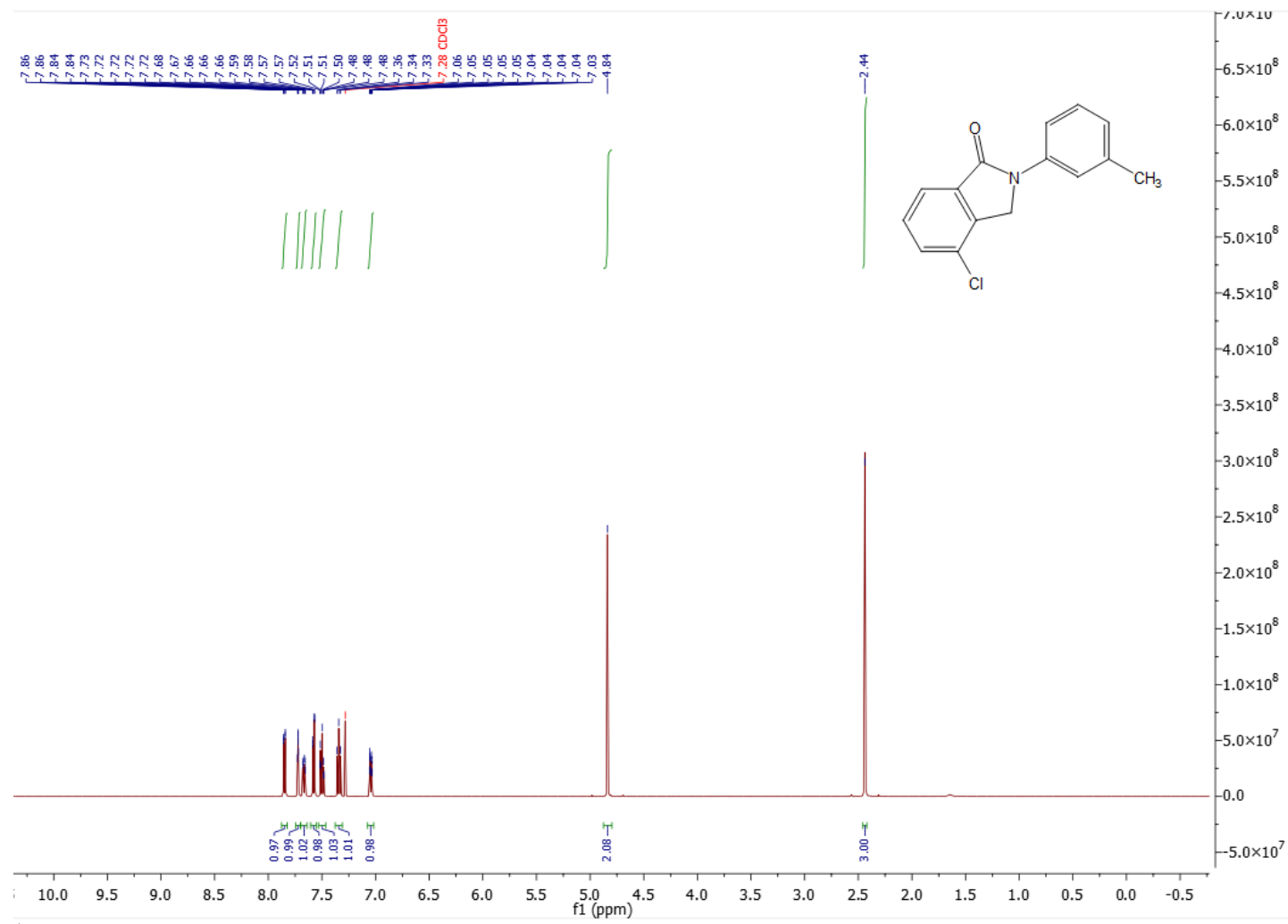

${ }^{1} \mathrm{H}$ NMR spectrum of compound $26\left(500 \mathrm{MHz}, \mathrm{CDCl}_{3}, \mathrm{rt}\right)$.

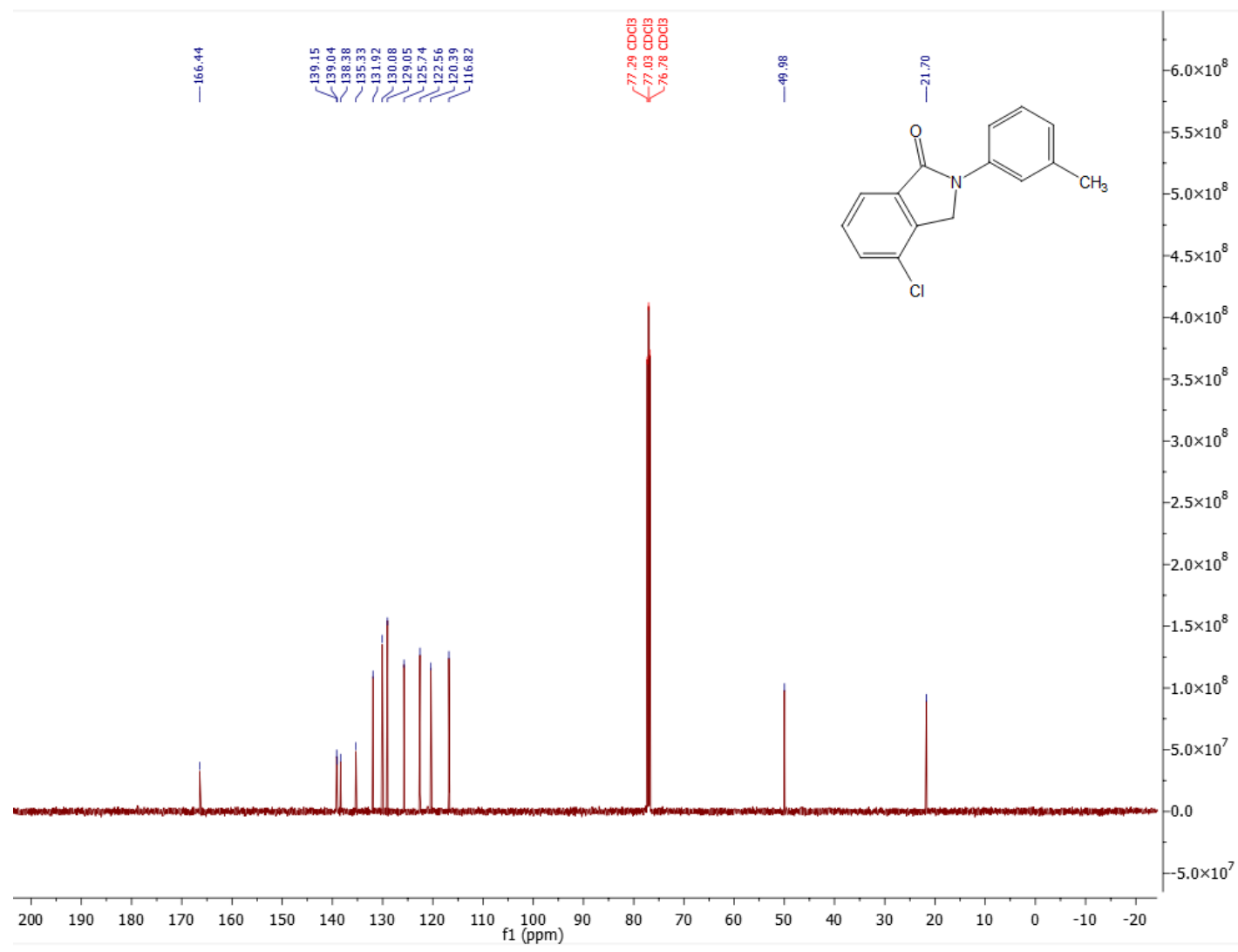

${ }^{13} \mathrm{C}$ NMR spectrum of compound $26\left(126 \mathrm{MHz}, \mathrm{CDCl}_{3}, \mathrm{rt}\right)$. 


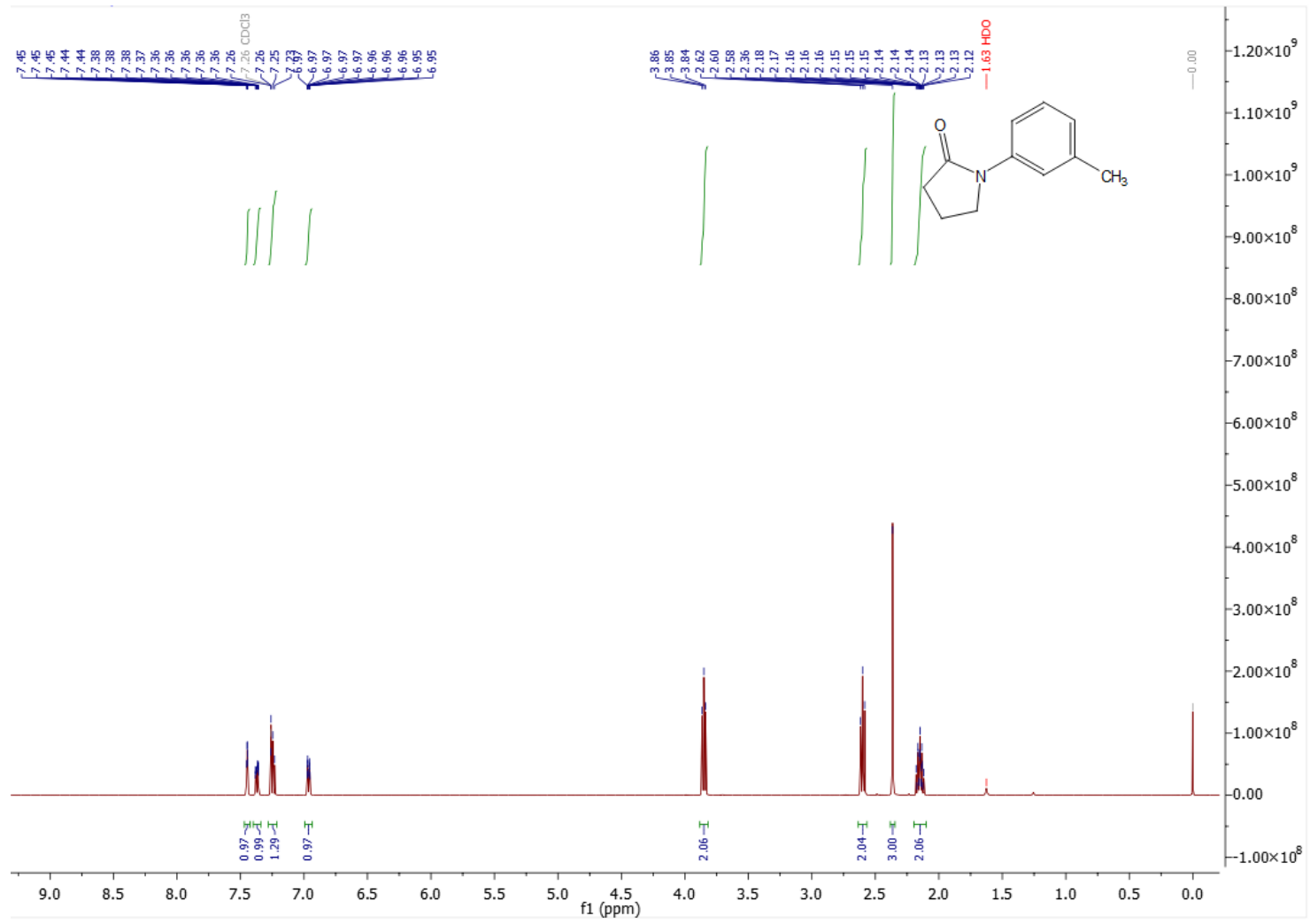

${ }^{1} \mathrm{H}$ NMR spectrum of compound $27\left(500 \mathrm{MHz}, \mathrm{CDCl}_{3}, \mathrm{rt}\right)$.

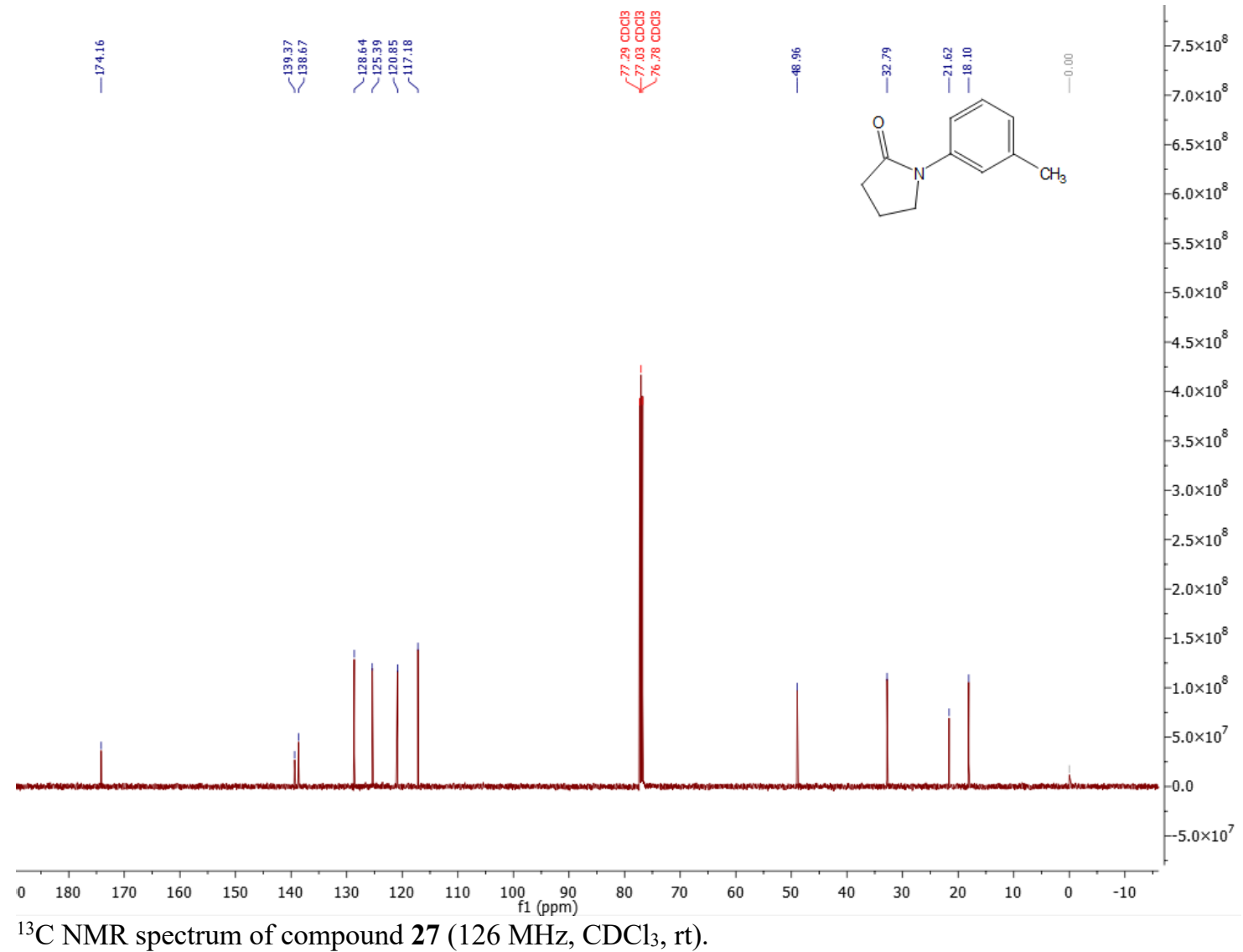




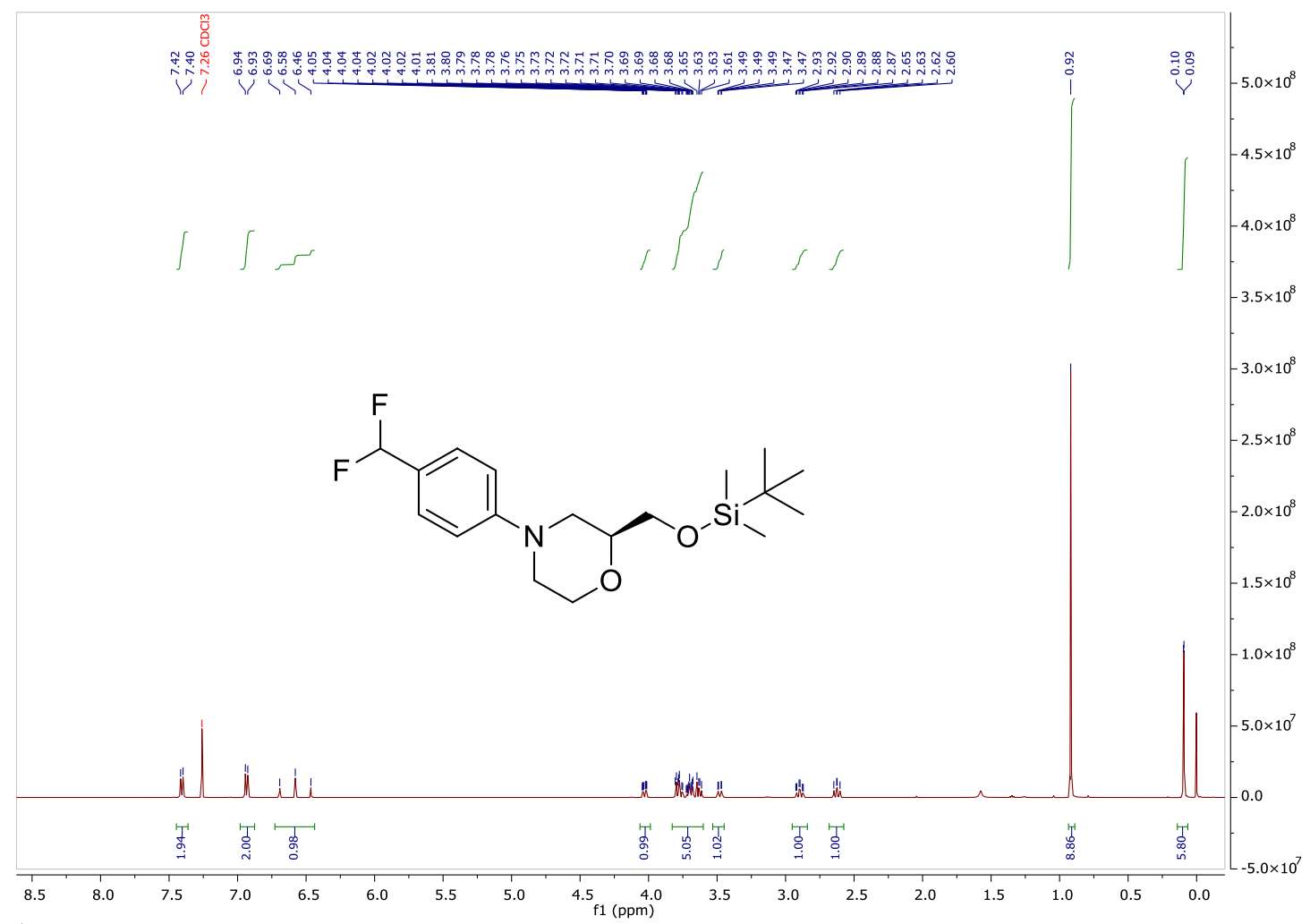

${ }^{1} \mathrm{H}$ NMR spectrum of compound $28\left(500 \mathrm{MHz}, \mathrm{CDCl}_{3}, \mathrm{rt}\right)$.

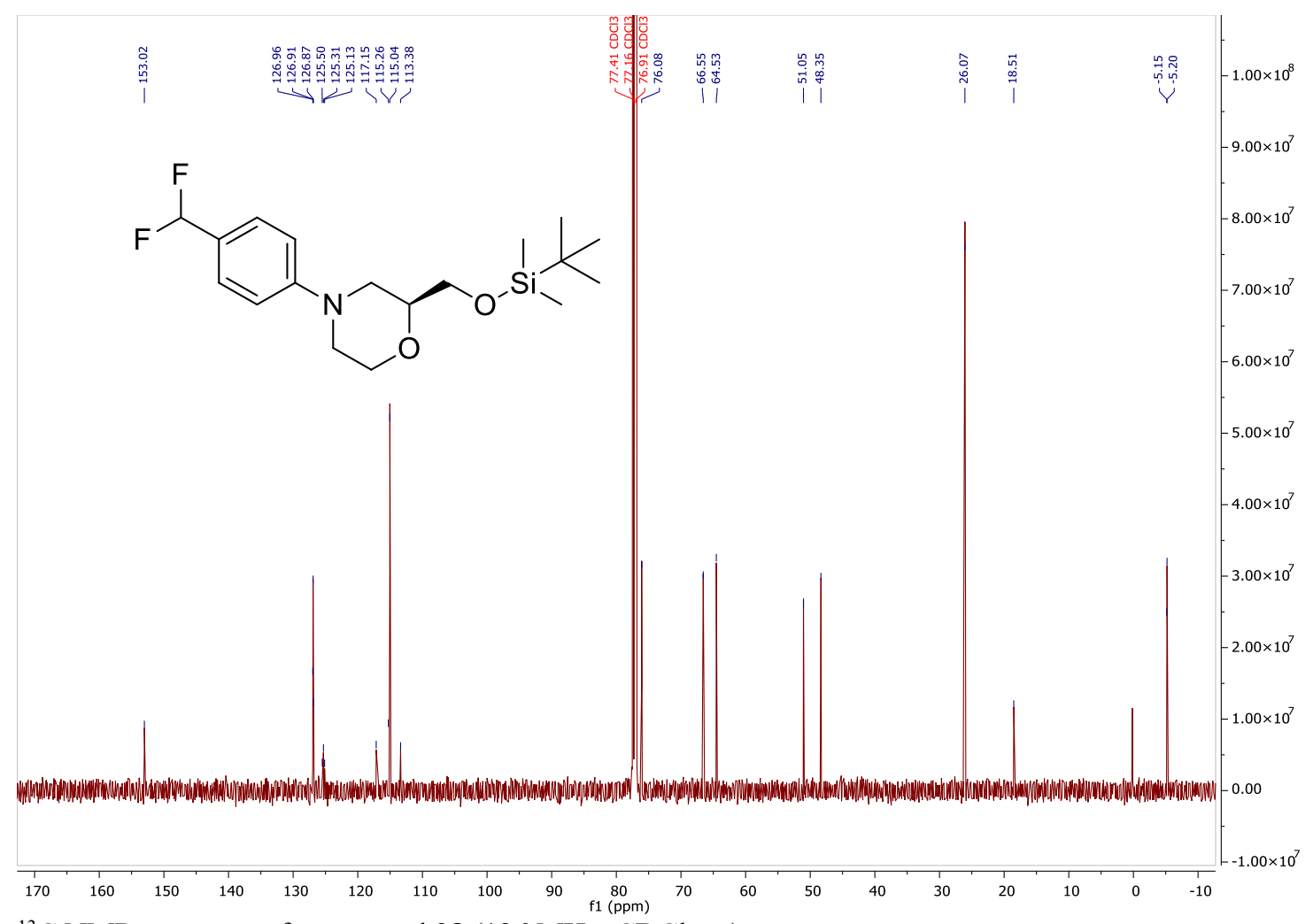

${ }^{13} \mathrm{C}$ NMR spectrum of compound $28\left(126 \mathrm{MHz}, \mathrm{CDCl}_{3}, \mathrm{rt}\right)$. 


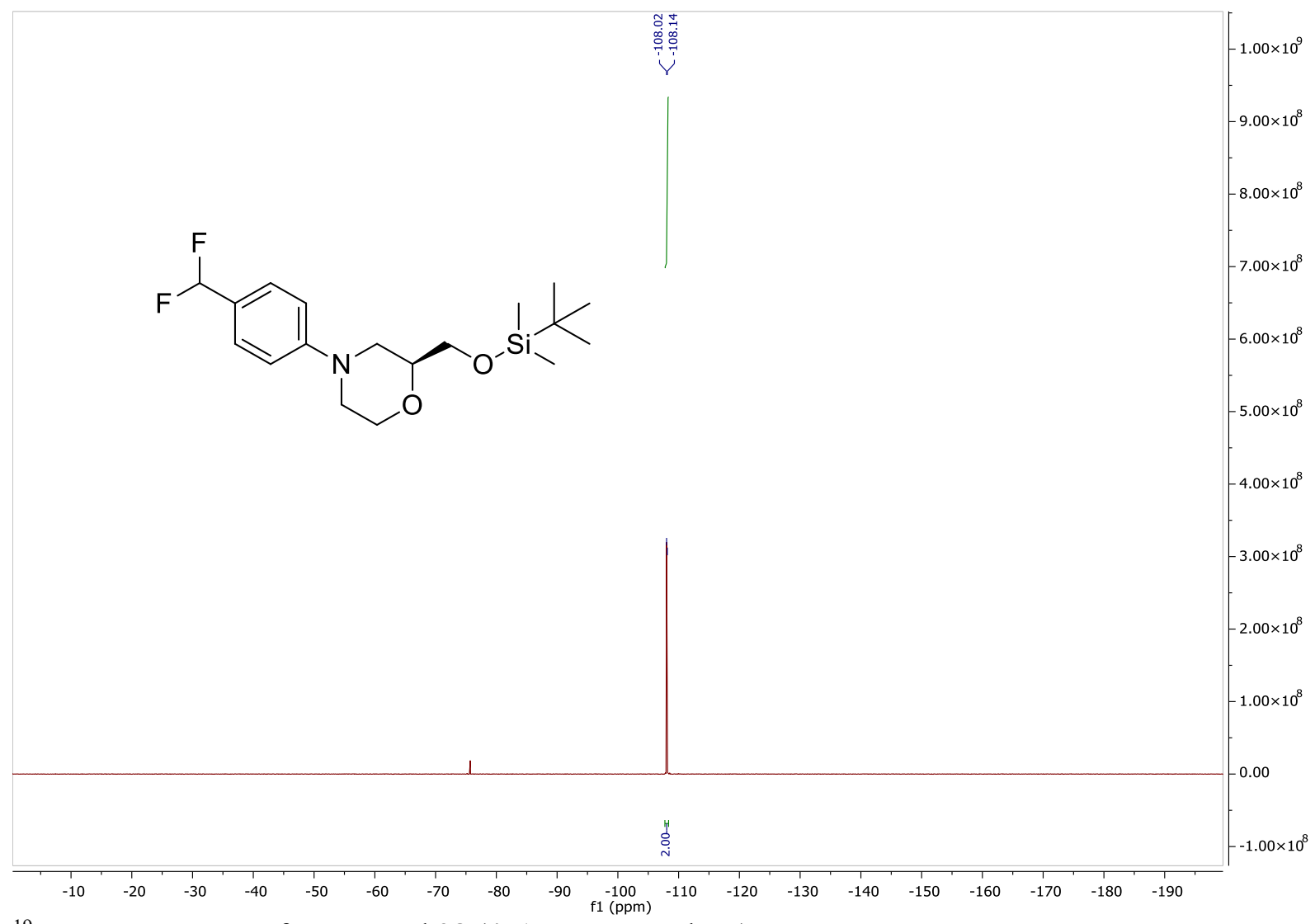

${ }^{19} \mathrm{~F}$ NMR spectrum of compound 28 (471 $\left.\mathrm{MHz}, \mathrm{CDCl}_{3}, \mathrm{rt}\right)$. 


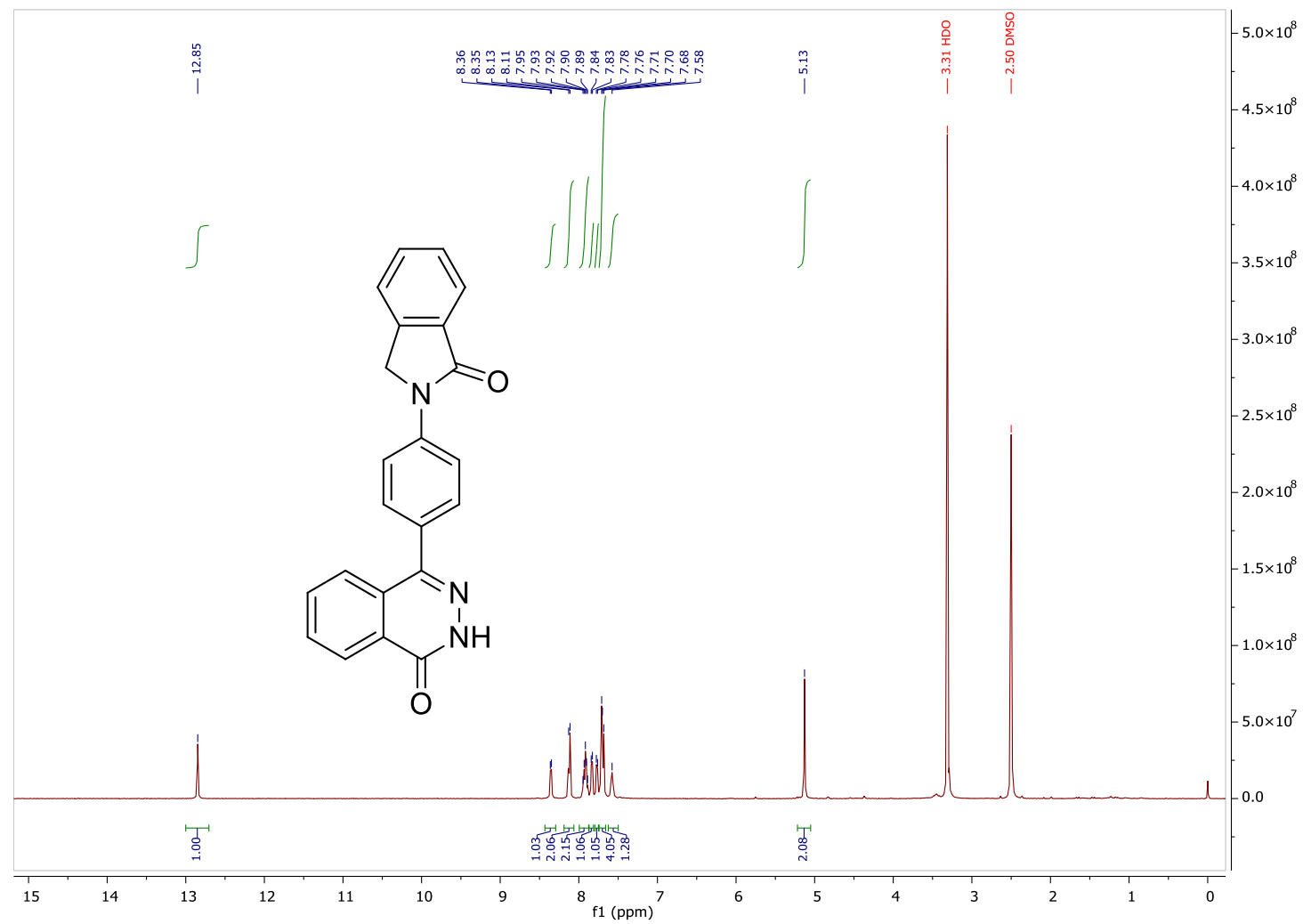

${ }^{1} \mathrm{H}$ NMR spectrum of compound 29 (500 MHz, DMSO- $\left.d_{6}, \mathrm{rt}\right)$.

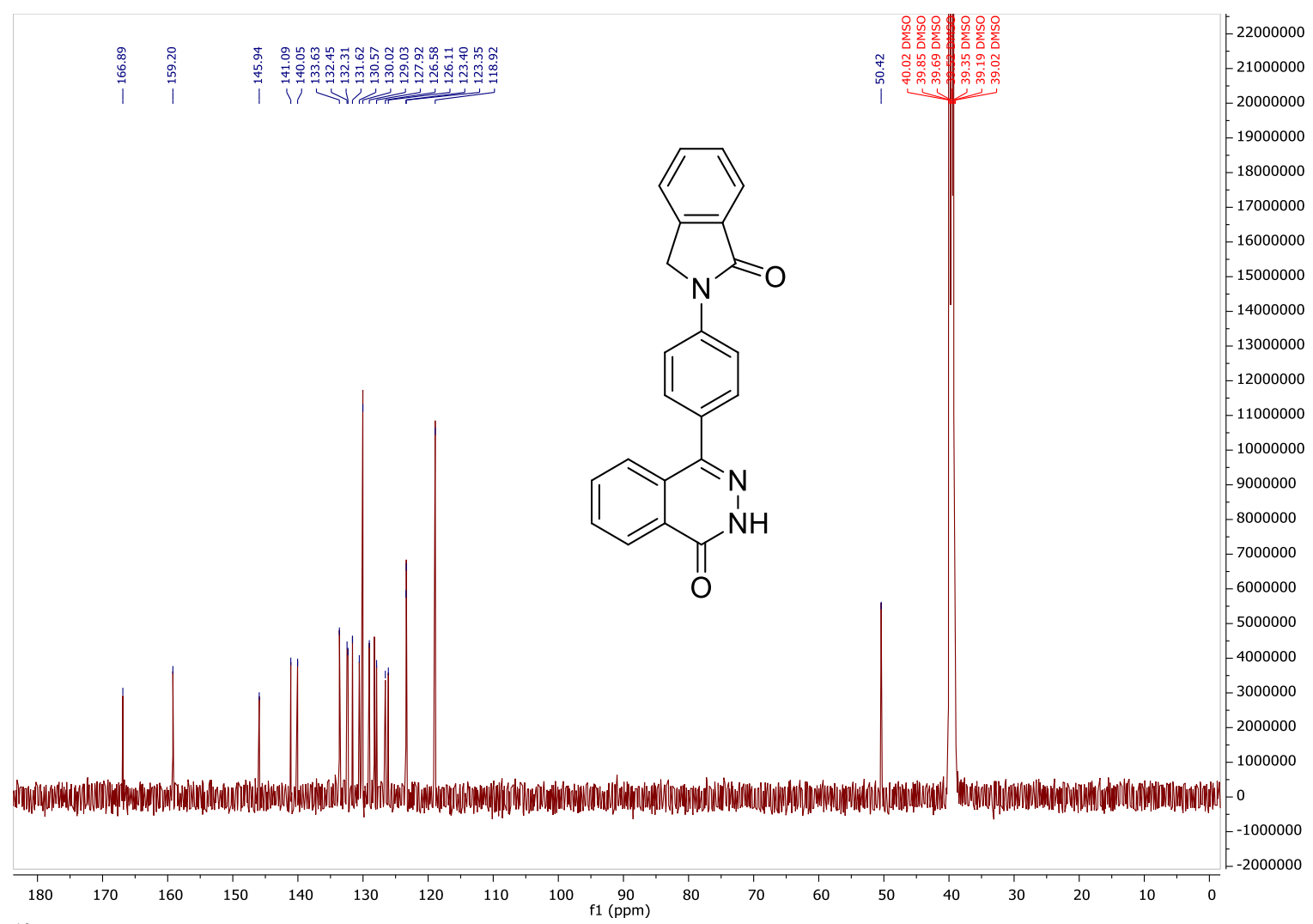

${ }^{13} \mathrm{C}$ NMR spectrum of compound 29 (126 MHz, DMSO- $\left.d_{6}, \mathrm{rt}\right)$. 


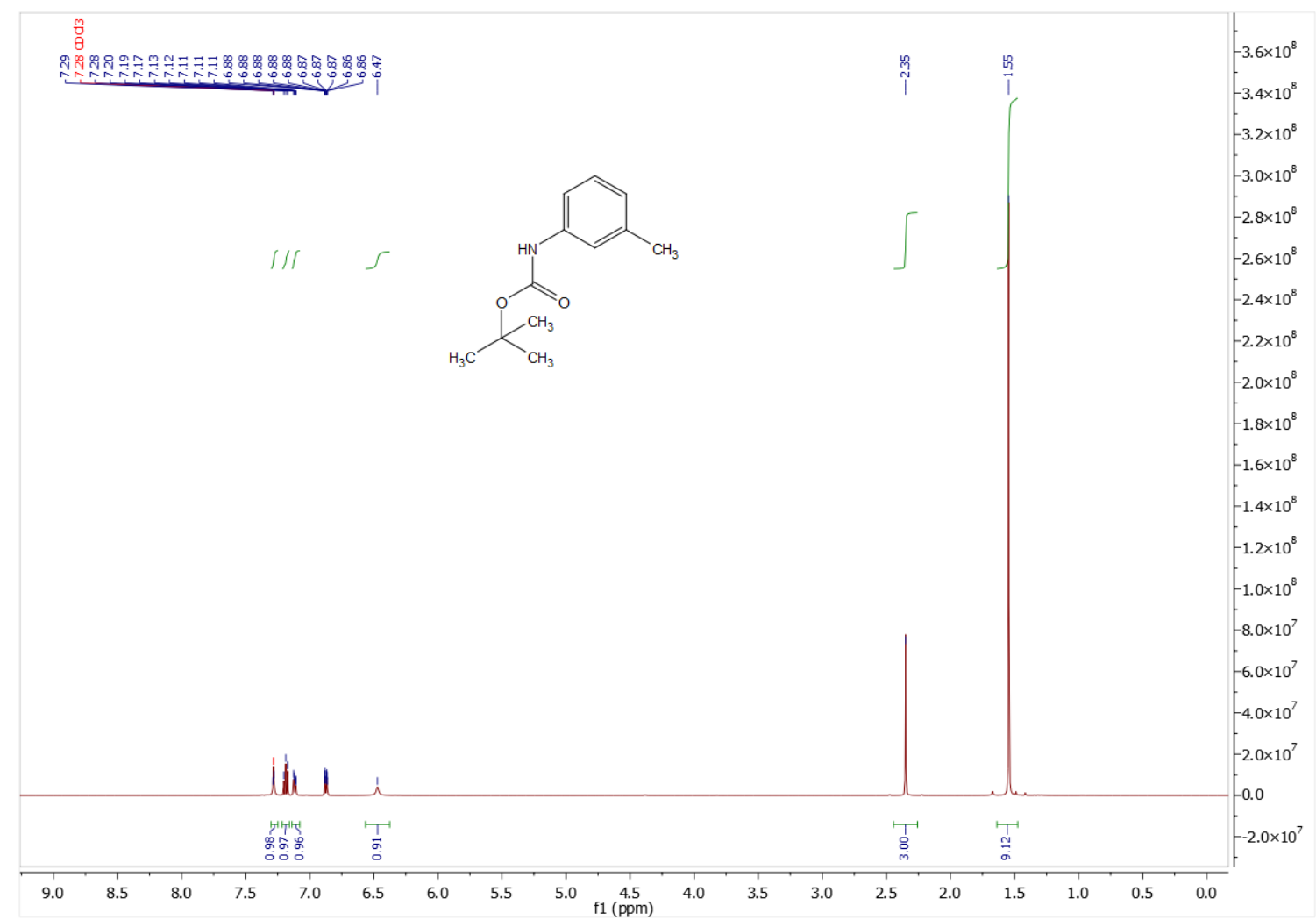

${ }^{1} \mathrm{H}$ NMR spectrum of compound $\mathbf{3 0}\left(500 \mathrm{MHz}, \mathrm{CDCl}_{3}, \mathrm{rt}\right)$.

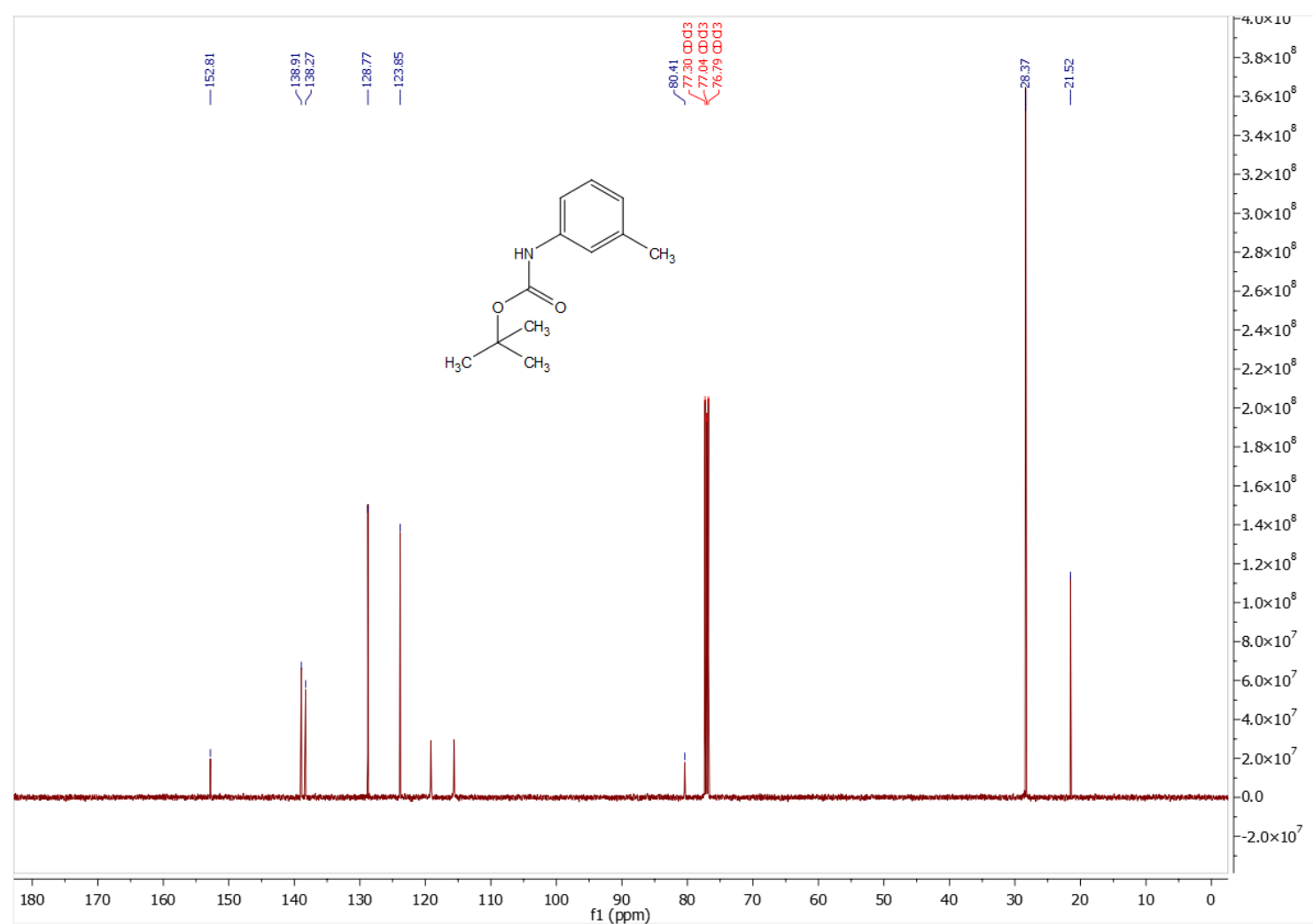

${ }^{13} \mathrm{C}$ NMR spectrum of compound $30\left(126 \mathrm{MHz}, \mathrm{CDCl}_{3}, \mathrm{rt}\right)$. 


许
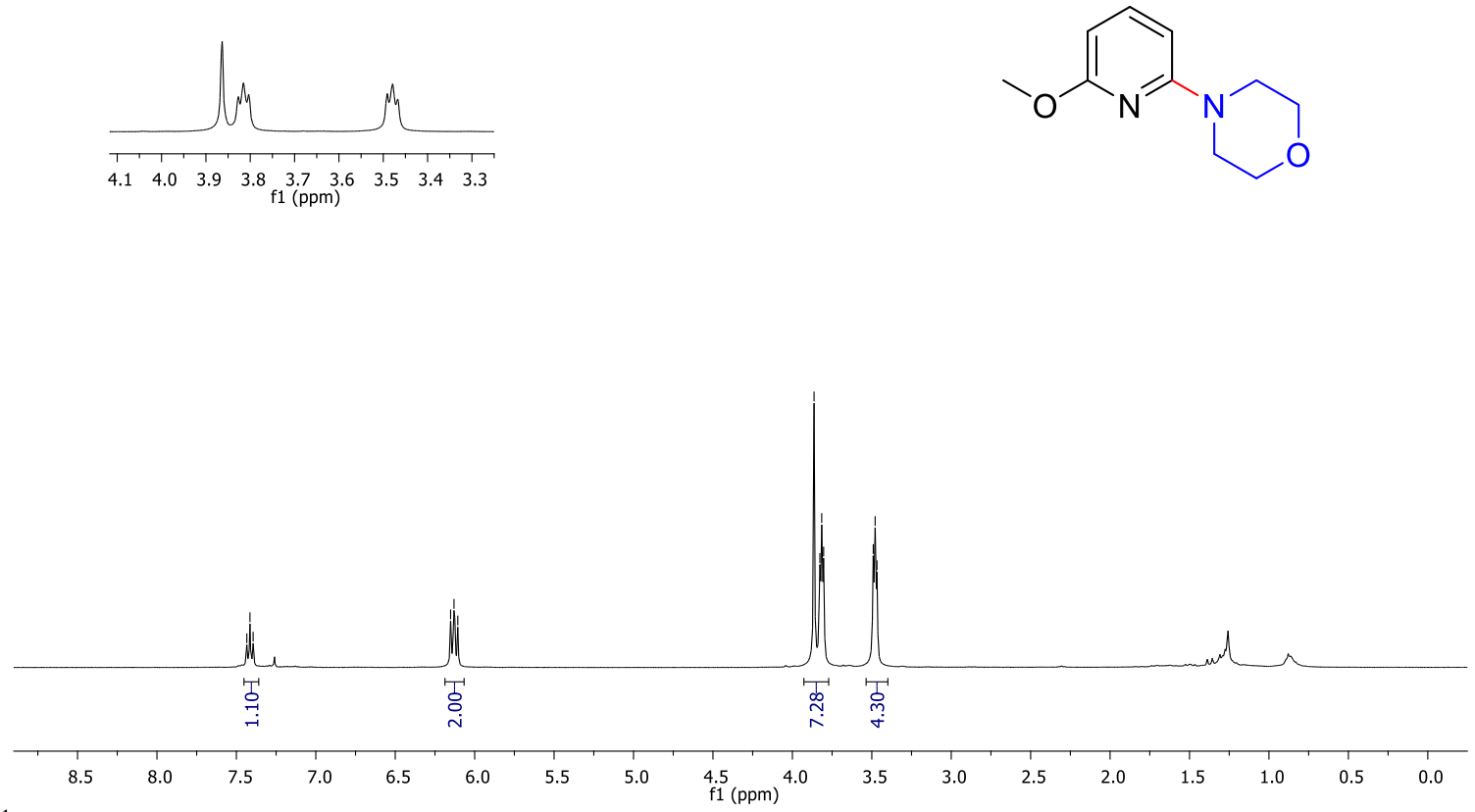

${ }^{1} \mathrm{H}$ NMR spectrum of compound $31\left(400 \mathrm{MHz}, \mathrm{CDCl}_{3}\right.$, rt).

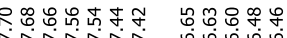

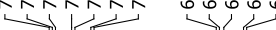

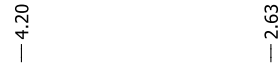
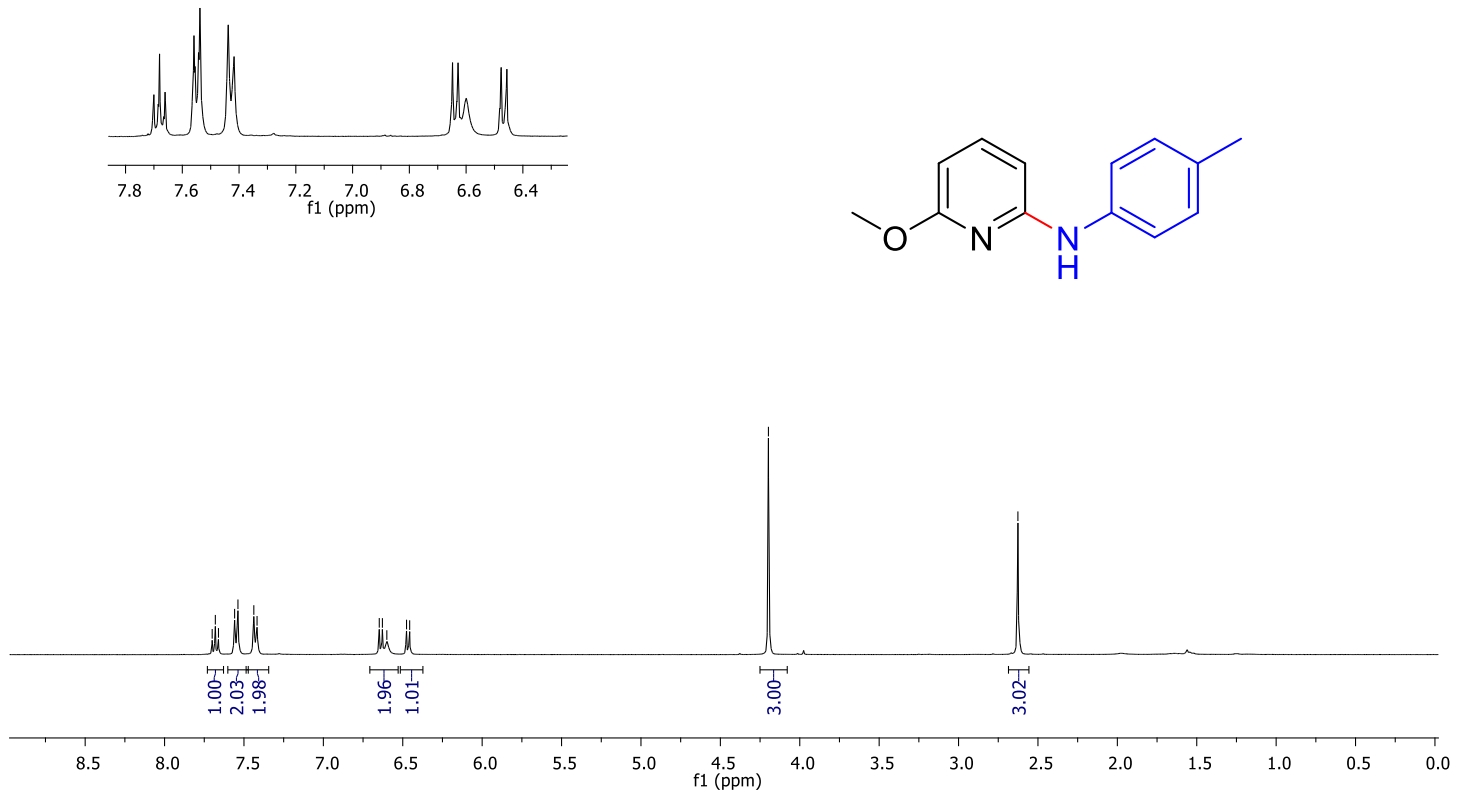

${ }^{1} \mathrm{H}$ NMR spectrum of compound $32\left(400 \mathrm{MHz}, \mathrm{CDCl}_{3}, \mathrm{rt}\right)$. 

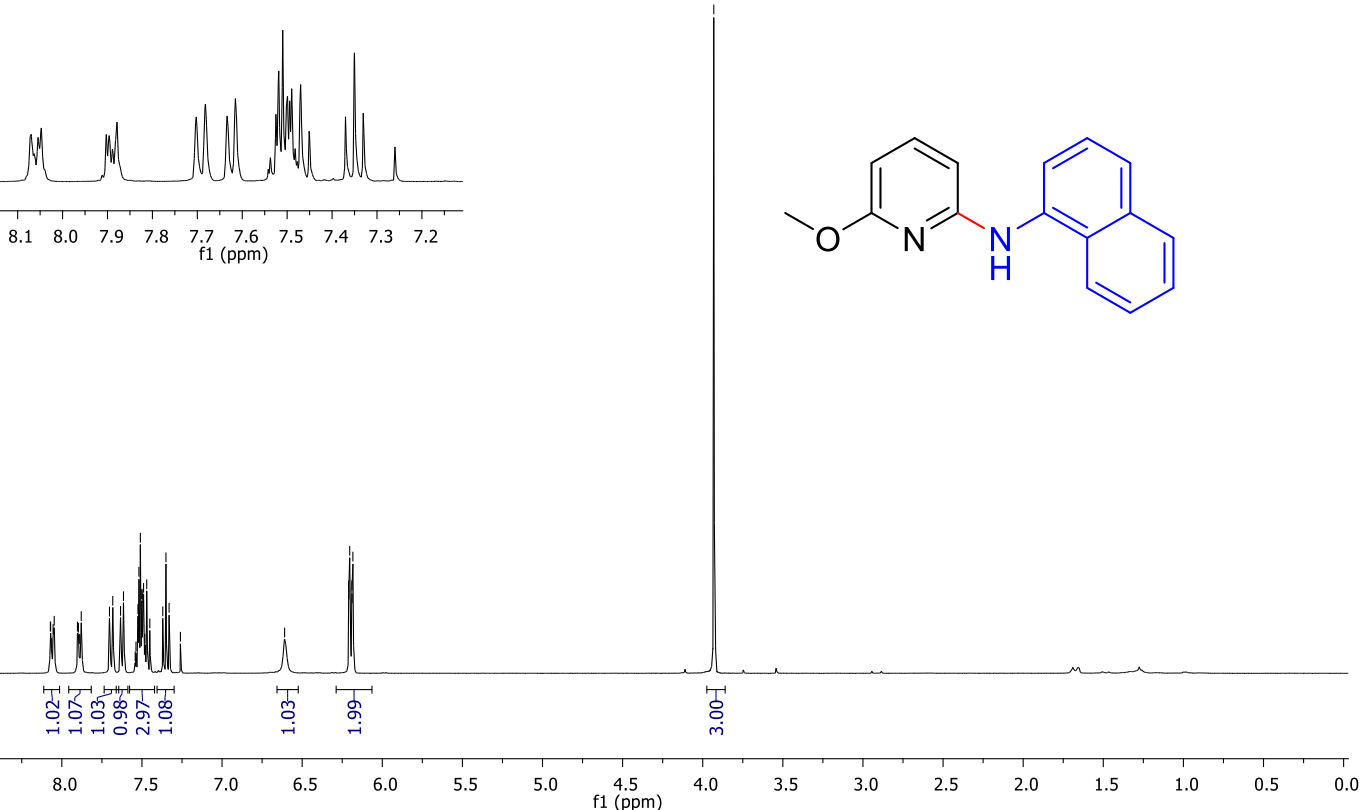

${ }^{1} \mathrm{H}$ NMR spectrum of compound $33\left(400 \mathrm{MHz}, \mathrm{CDCl}_{3}, \mathrm{rt}\right)$.
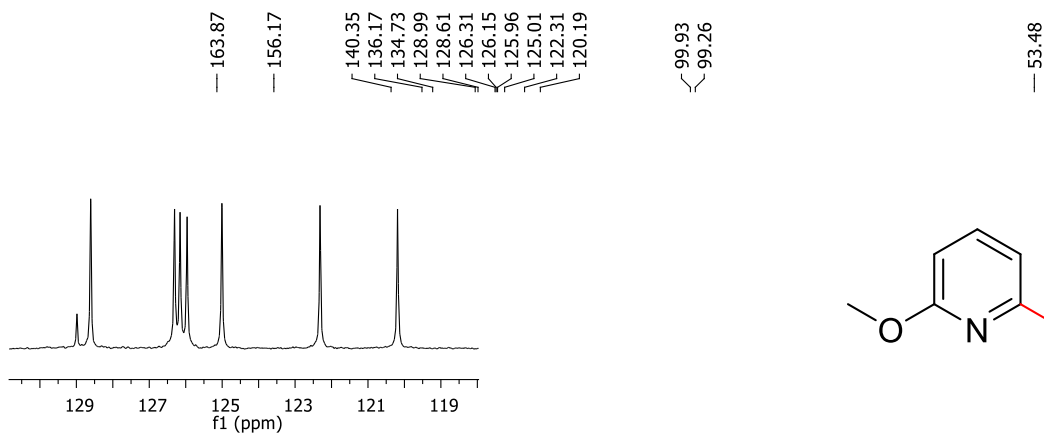<smiles>COc1cccc(Nc2cccc3ccccc23)n1</smiles>

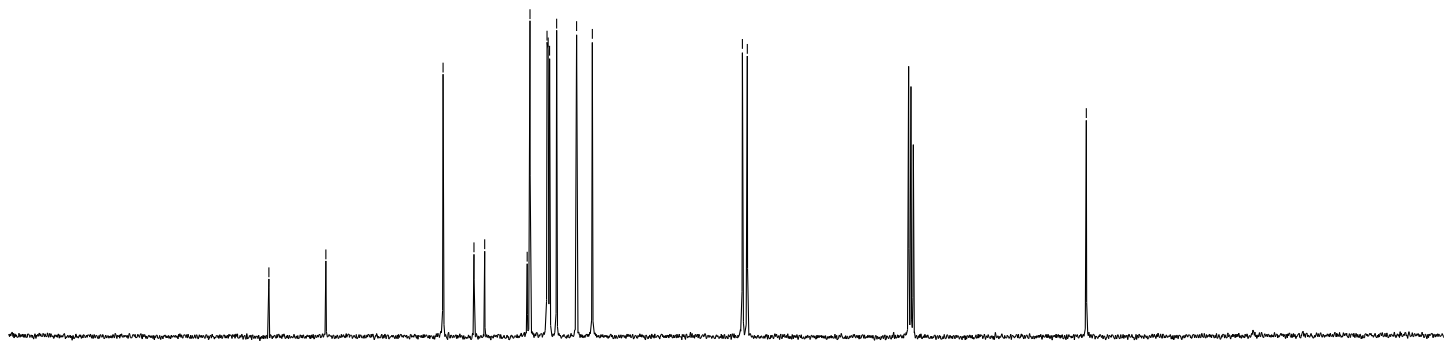

190

${ }^{13} \mathrm{C}$ NMR spectrum of compound $33\left(100 \mathrm{MHz}, \mathrm{CDCl}_{3}, \mathrm{rt}\right)$. 

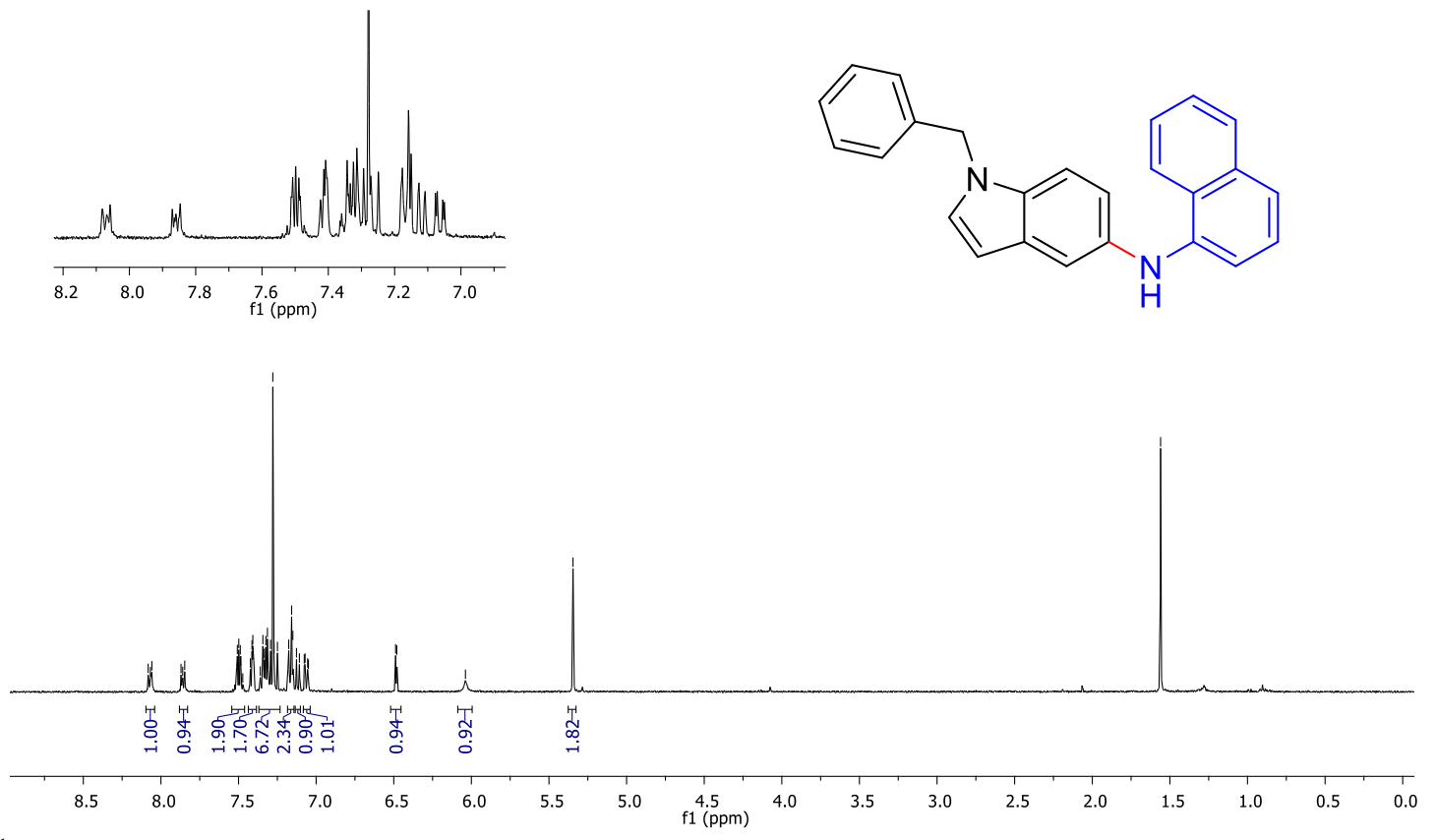

${ }^{1} \mathrm{H}$ NMR spectrum of compound $34\left(400 \mathrm{MHz}, \mathrm{CDCl}_{3}\right.$, rt).

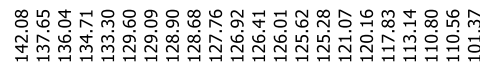

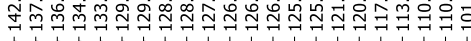

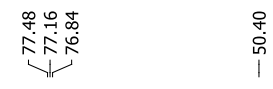
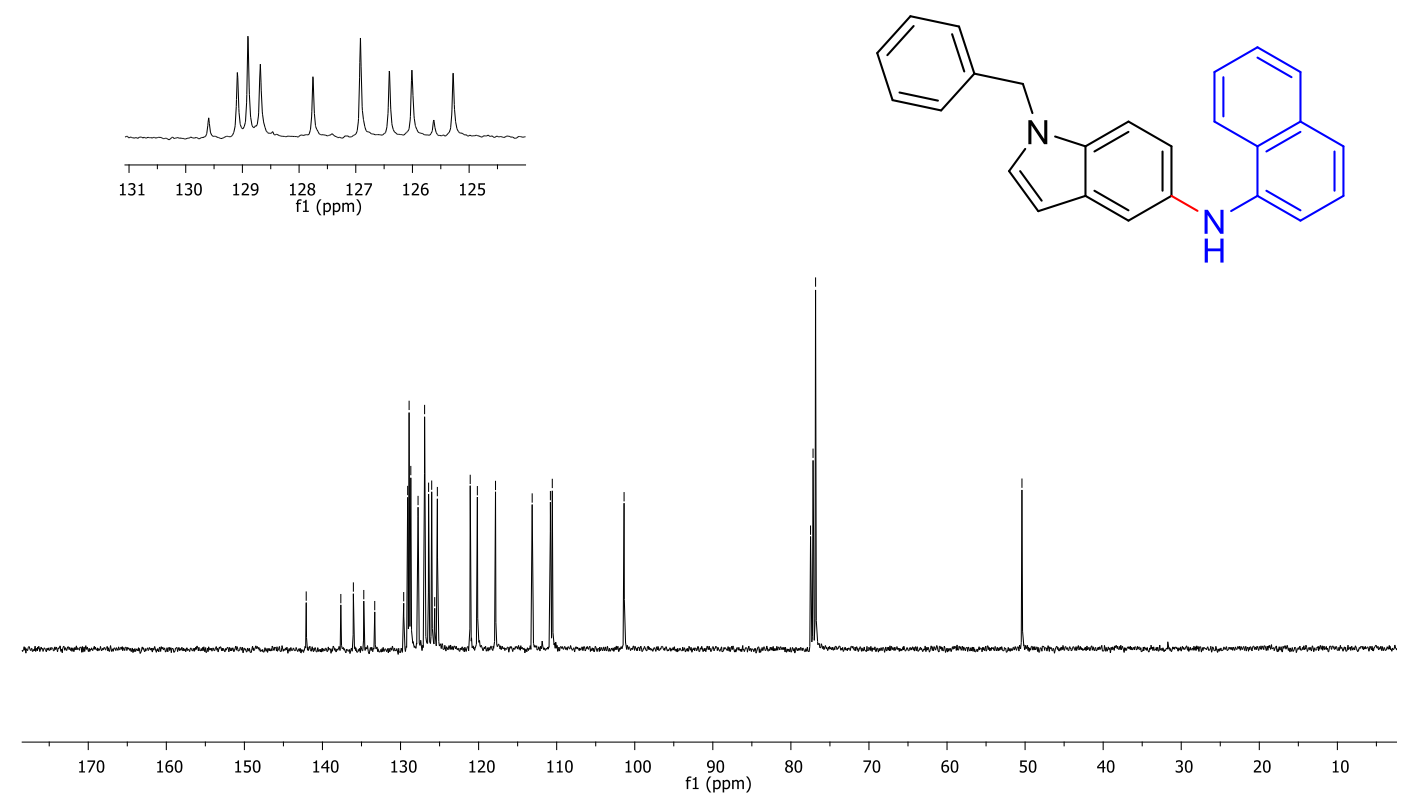

${ }^{13} \mathrm{C}$ NMR spectrum of compound $\mathbf{3 4}\left(100 \mathrm{MHz}, \mathrm{CDCl}_{3}, \mathrm{rt}\right)$. 


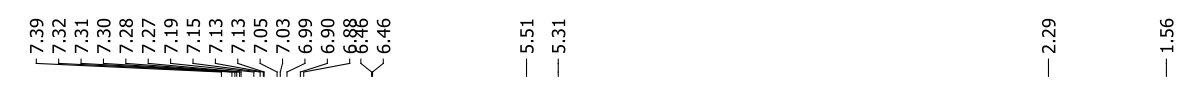
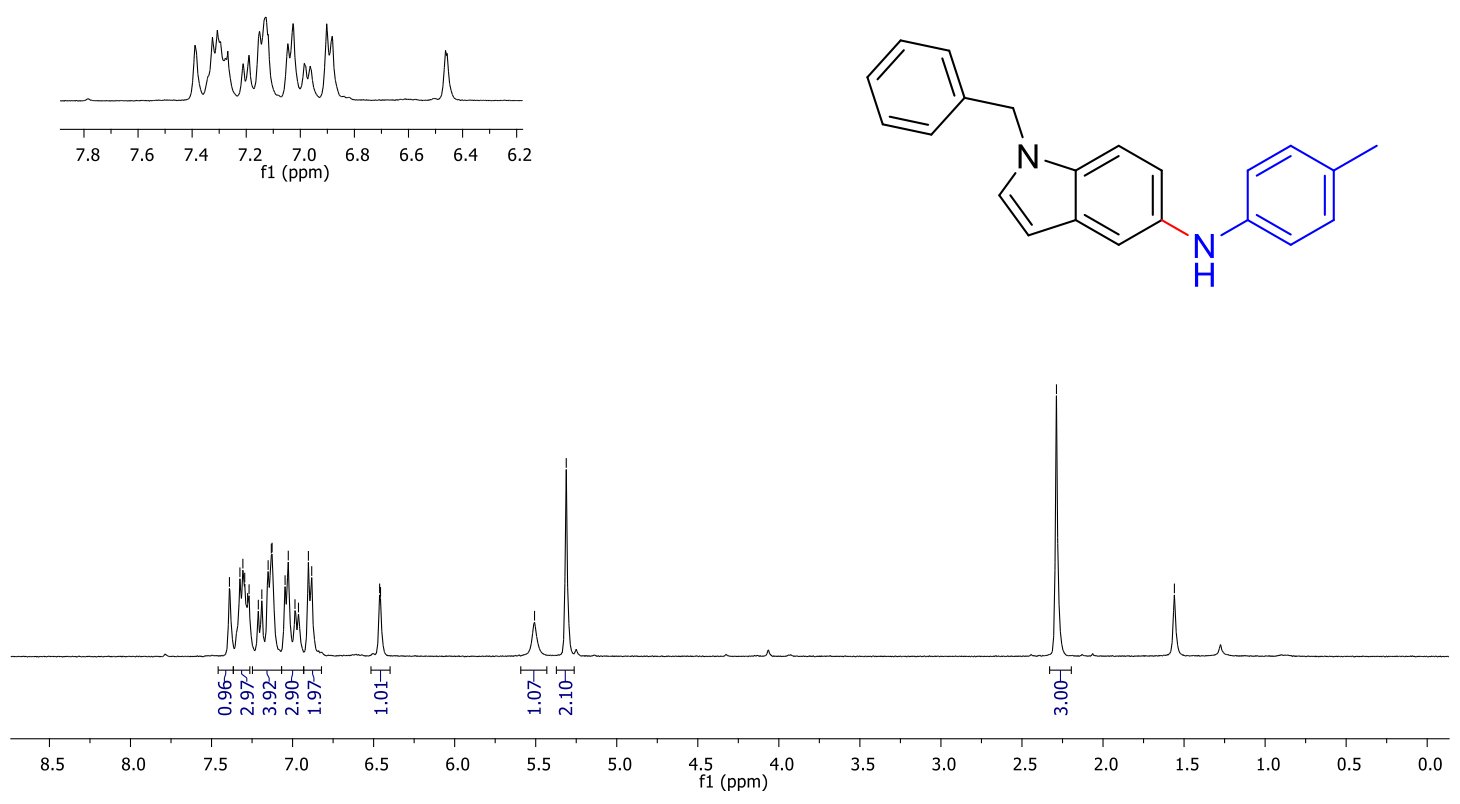

${ }^{1} \mathrm{H}$ NMR spectrum of compound 35 (400 MHz, $\left.\mathrm{CDCl}_{3}, \mathrm{rt}\right)$.
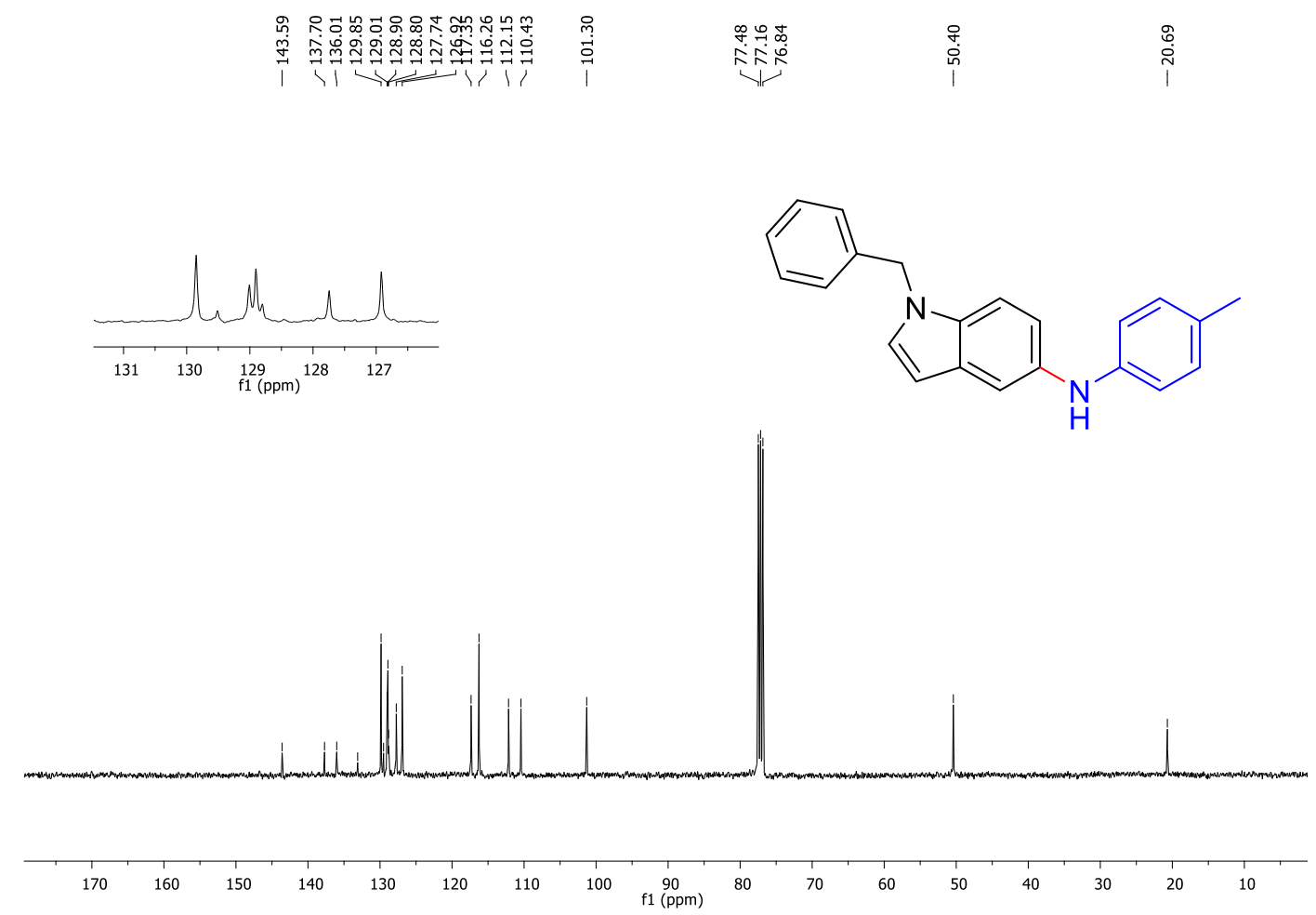

${ }^{13} \mathrm{C}$ NMR spectrum of compound $35\left(100 \mathrm{MHz}, \mathrm{CDCl}_{3}, \mathrm{rt}\right)$. 
14. Spectral Data of Amide Coupling Products
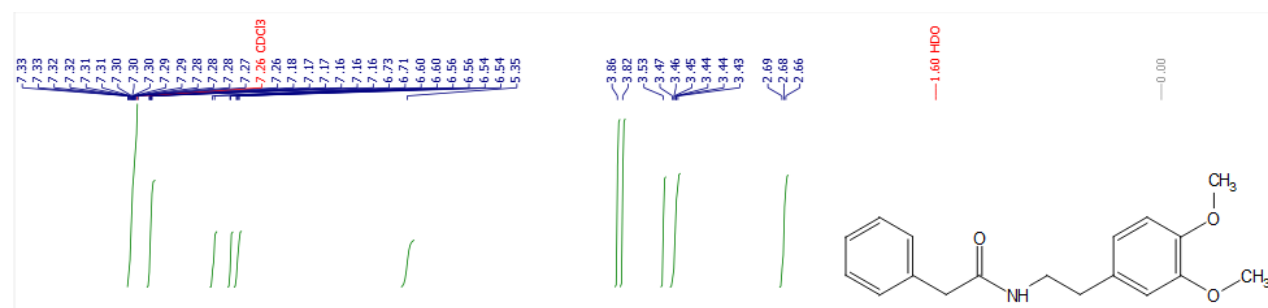

$3.0 \times 10$

$5.0 \times 10^{8}$

$10 \times 10$

$4.5 \times 10^{8}$

$-4.0 \times 10^{8}$

$-3.5 \times 10^{8}$

$3.0 \times 10^{8}$

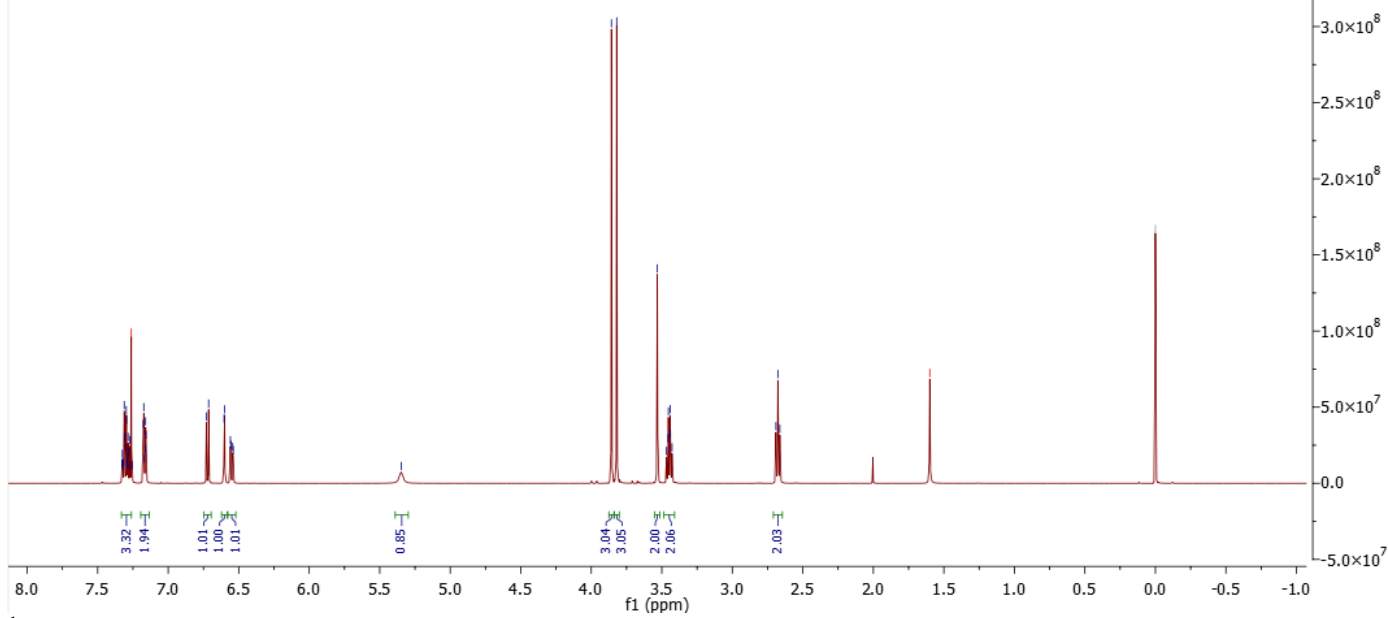

${ }^{1} \mathrm{H}$ NMR spectrum of compound $36\left(500 \mathrm{MHz}, \mathrm{CDCl}_{3}, \mathrm{rt}\right)$.

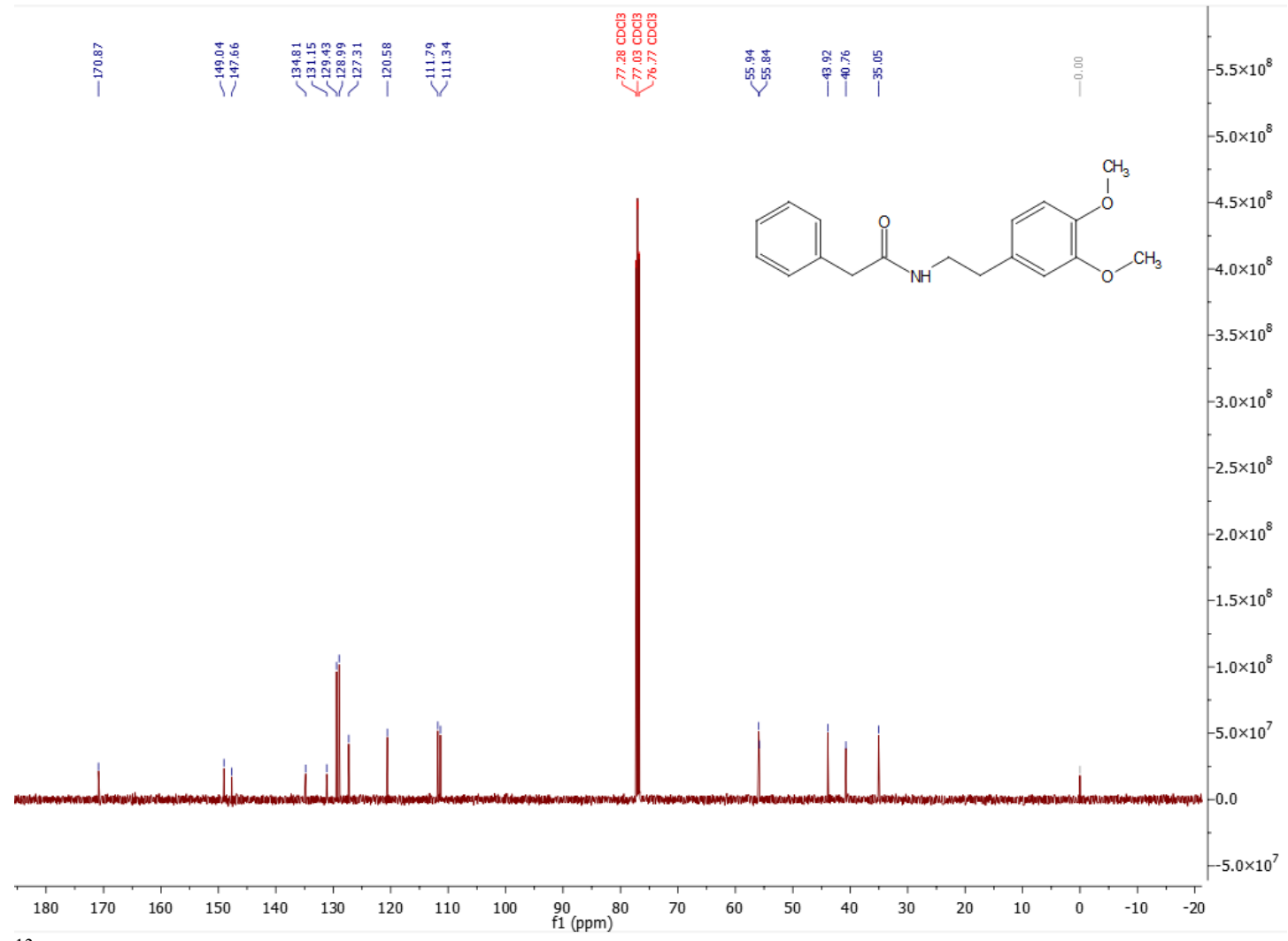

${ }^{13} \mathrm{C}$ NMR spectrum of compound $36\left(126 \mathrm{MHz}, \mathrm{CDCl}_{3}, \mathrm{rt}\right)$. 


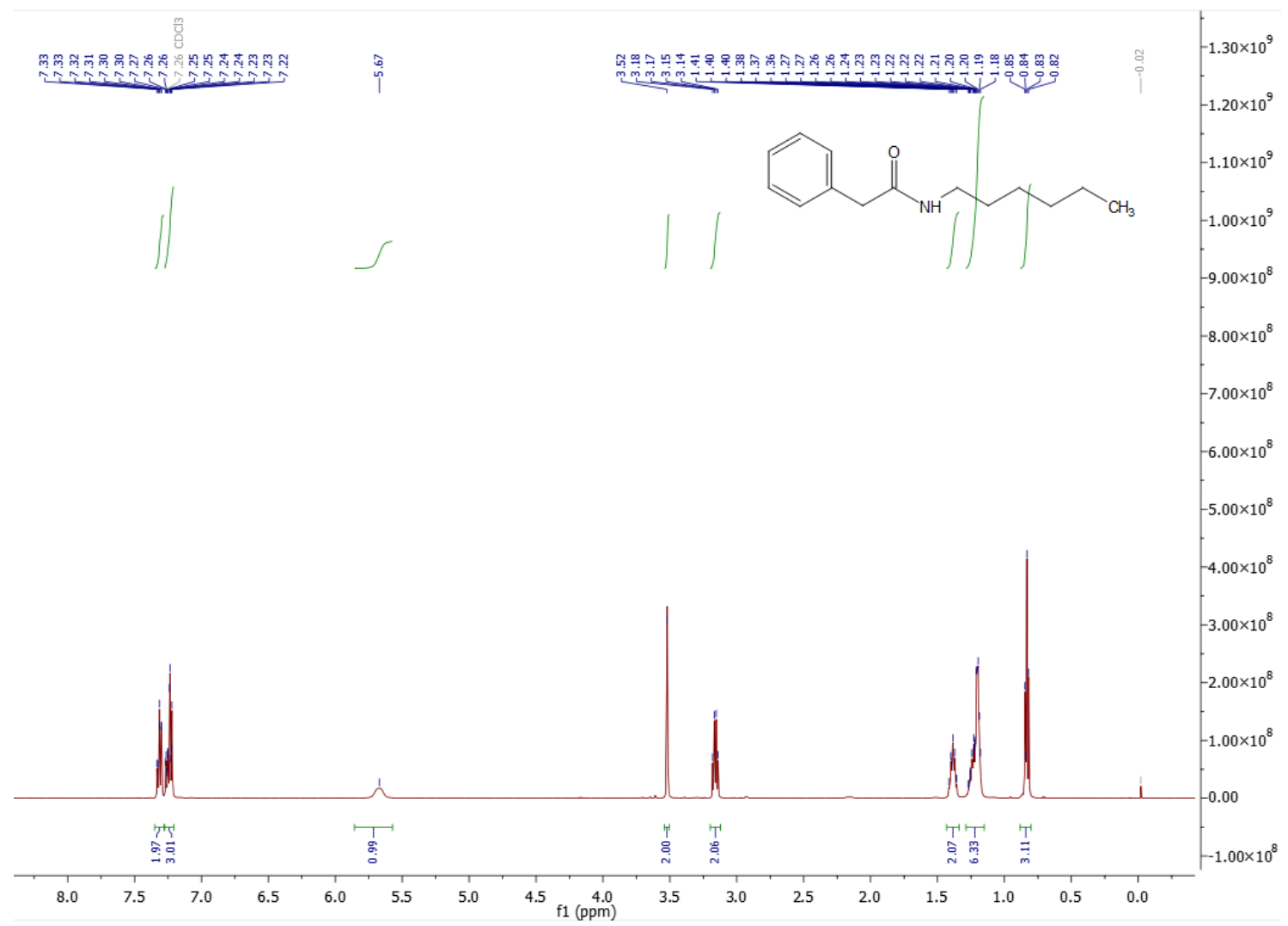

${ }^{1} \mathrm{H}$ NMR spectrum of compound $37\left(500 \mathrm{MHz}, \mathrm{CDCl}_{3}, \mathrm{rt}\right)$.

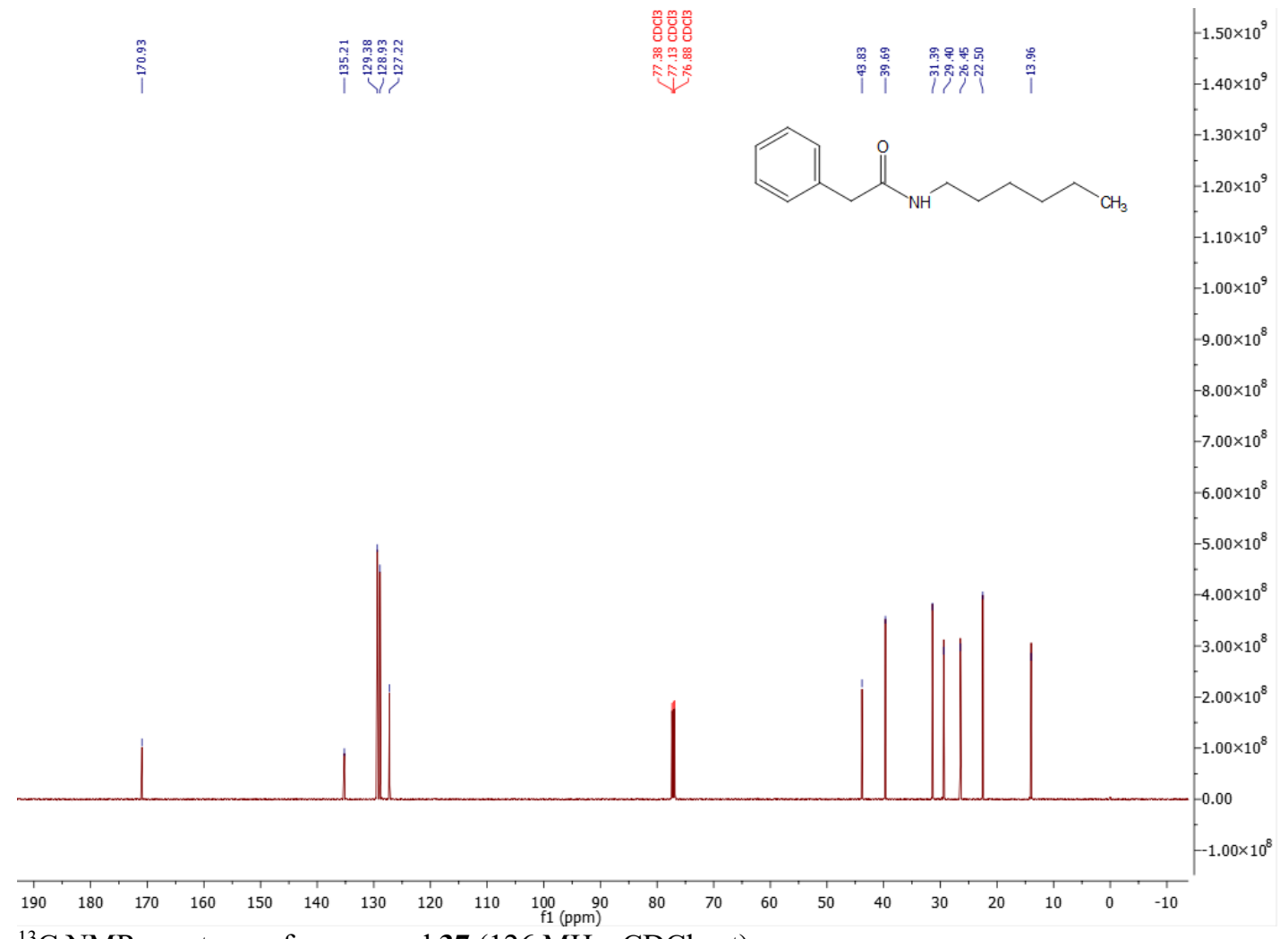

${ }^{13} \mathrm{C}$ NMR spectrum of compound $37\left(126 \mathrm{MHz}, \mathrm{CDCl}_{3}, \mathrm{rt}\right)$. 


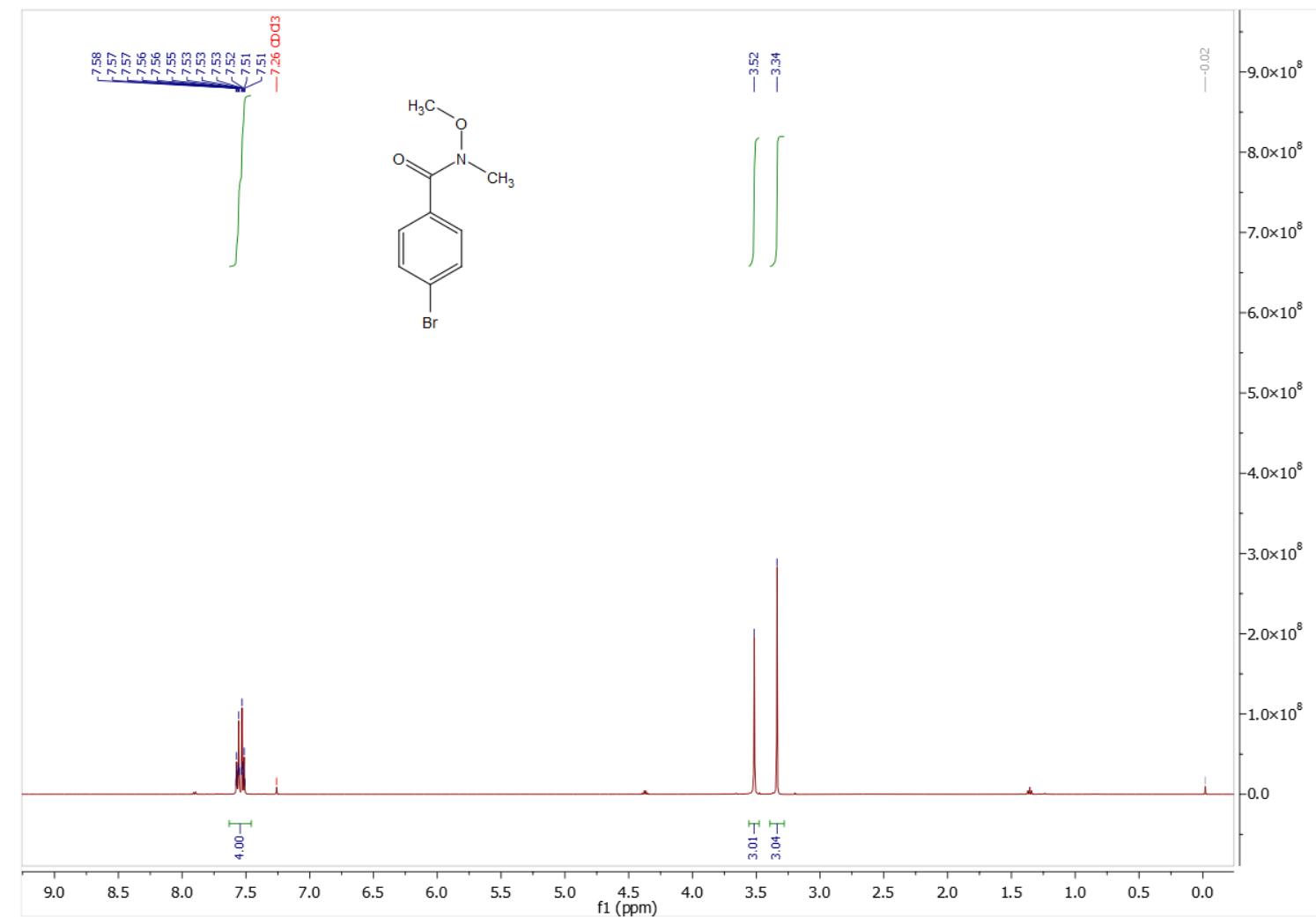

${ }^{1} \mathrm{H}$ NMR spectrum of compound $39\left(500 \mathrm{MHz}, \mathrm{CDCl}_{3}, \mathrm{rt}\right)$.

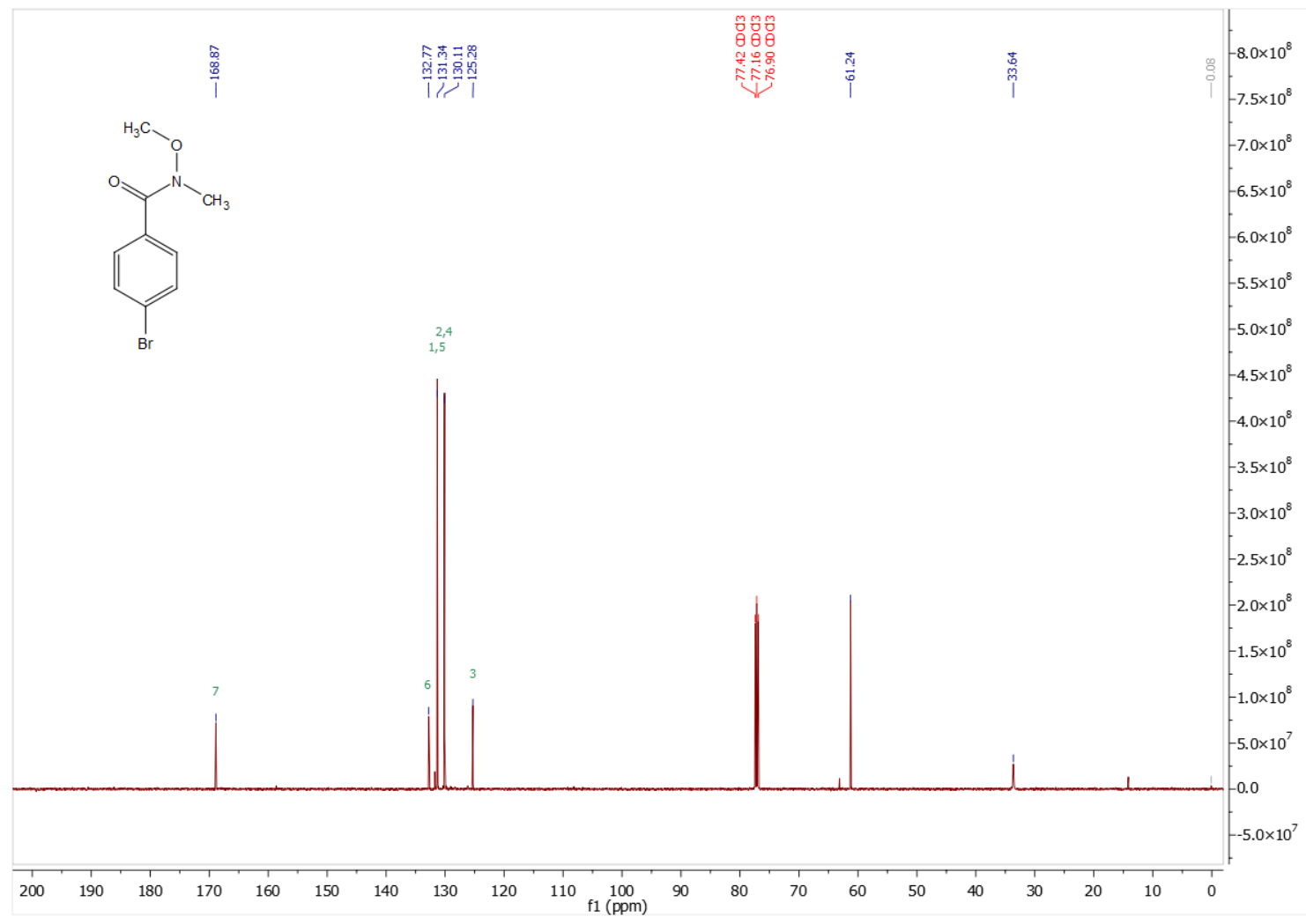

${ }^{13} \mathrm{C}$ NMR spectrum of compound $39\left(126 \mathrm{MHz}, \mathrm{CDCl}_{3}, \mathrm{rt}\right)$. 


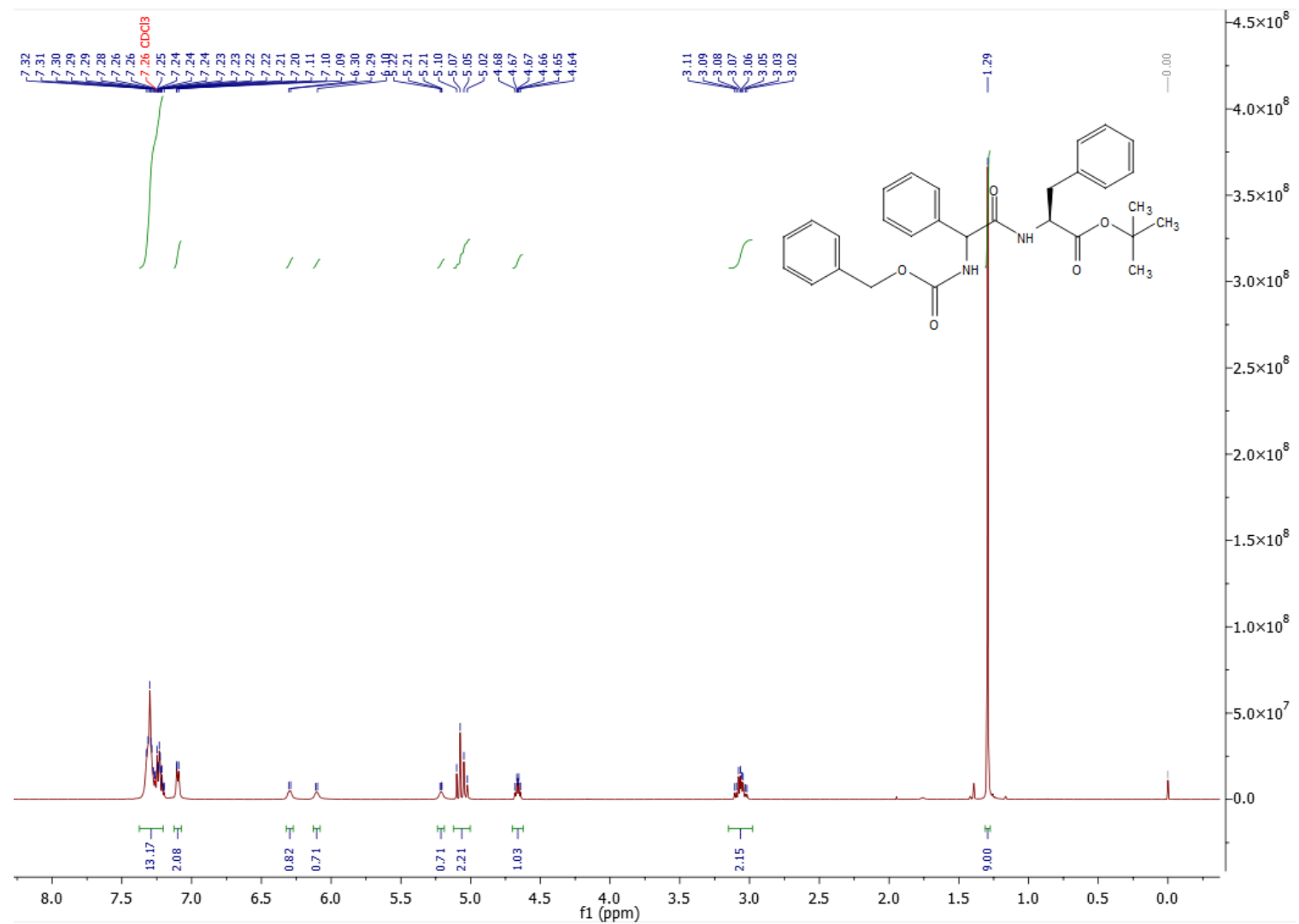

${ }^{1} \mathrm{H}$ NMR spectrum of compound $40\left(500 \mathrm{MHz}, \mathrm{CDCl}_{3}, \mathrm{rt}\right)$.

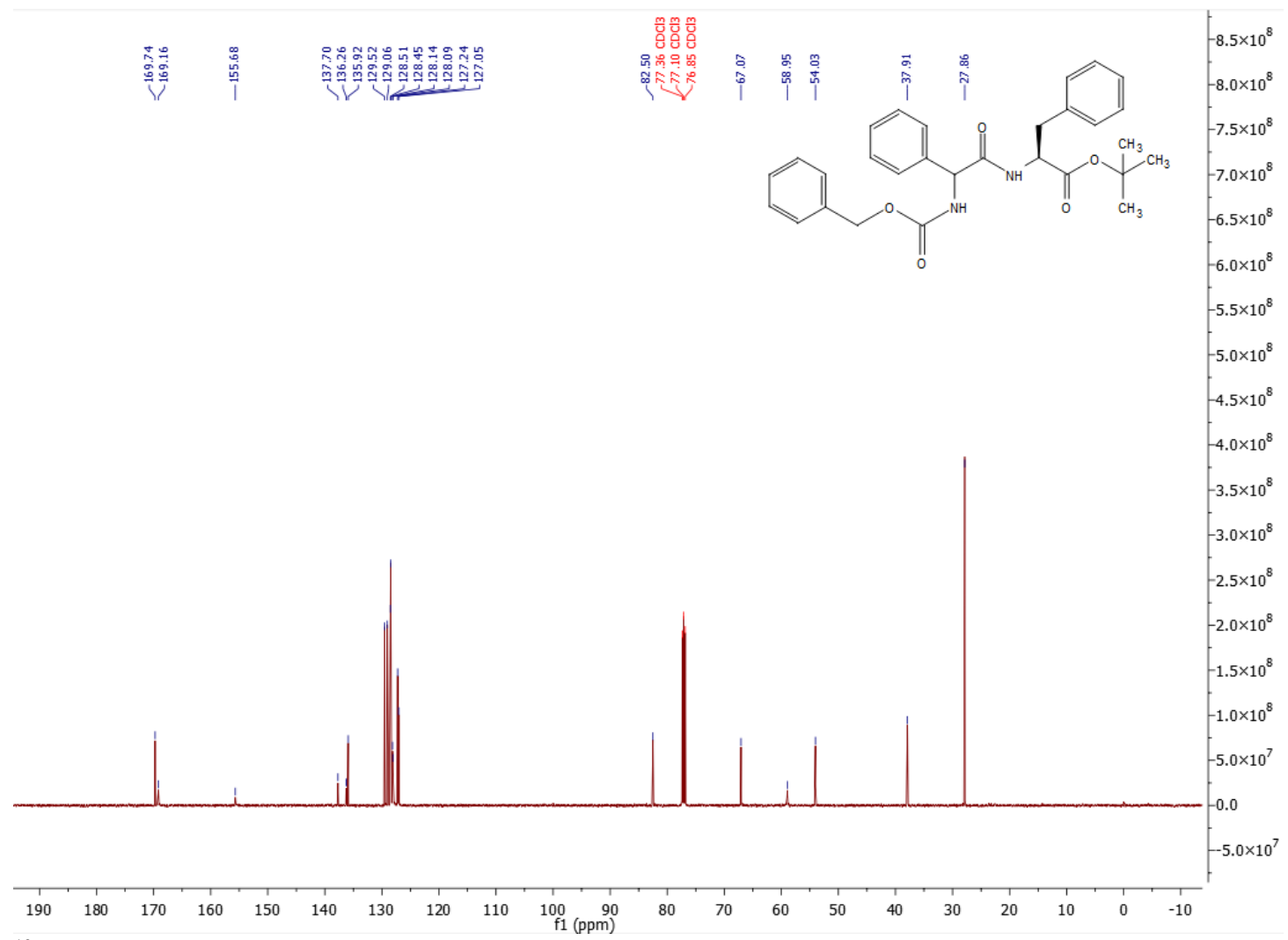

${ }^{13} \mathrm{C}$ NMR spectrum of compound $40\left(126 \mathrm{MHz}, \mathrm{CDCl}_{3}, \mathrm{rt}\right)$. 


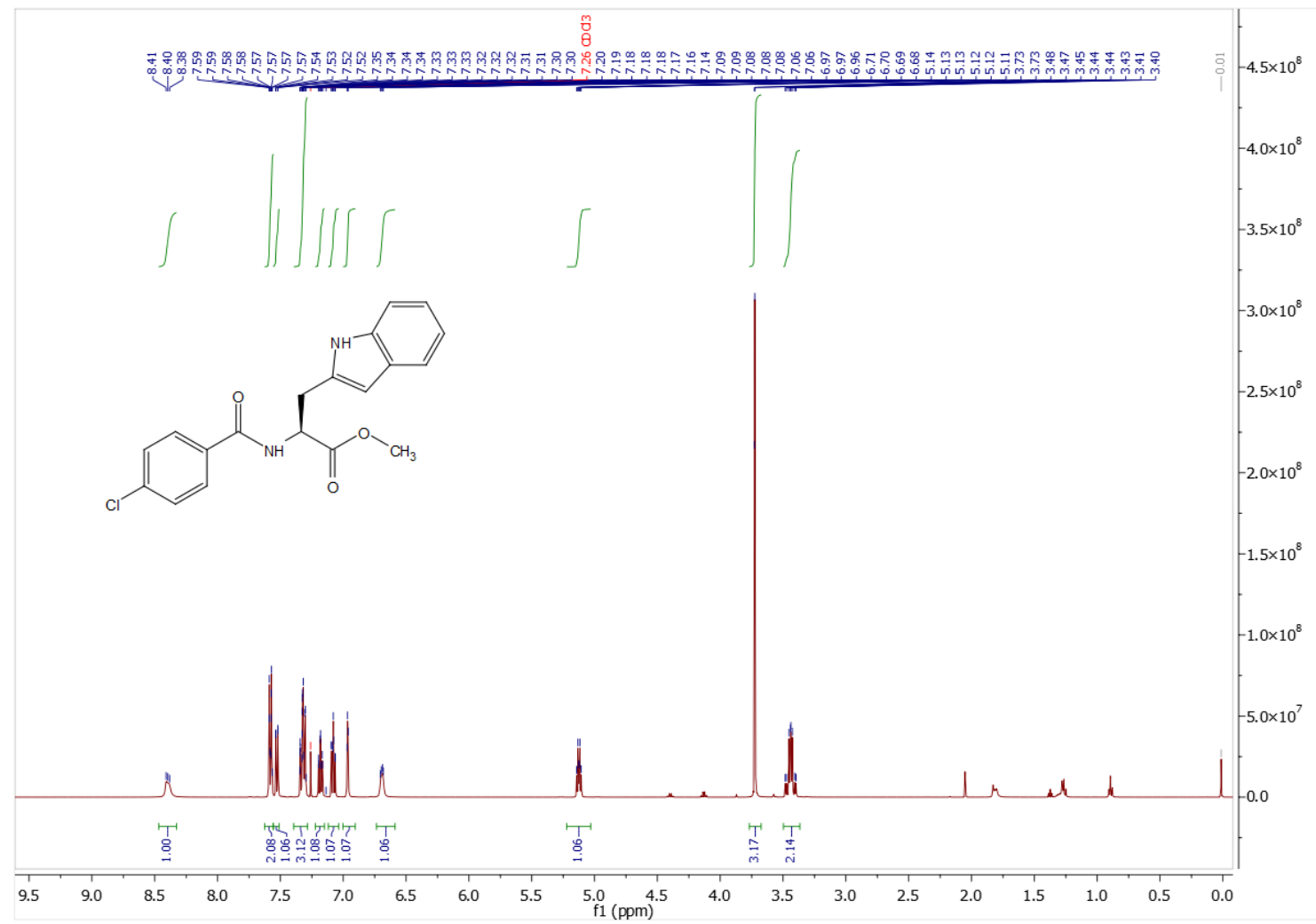

${ }^{1} \mathrm{H}$ NMR spectrum of compound 41 (500 MHz, $\left.\mathrm{CDCl}_{3}, \mathrm{rt}\right)$.

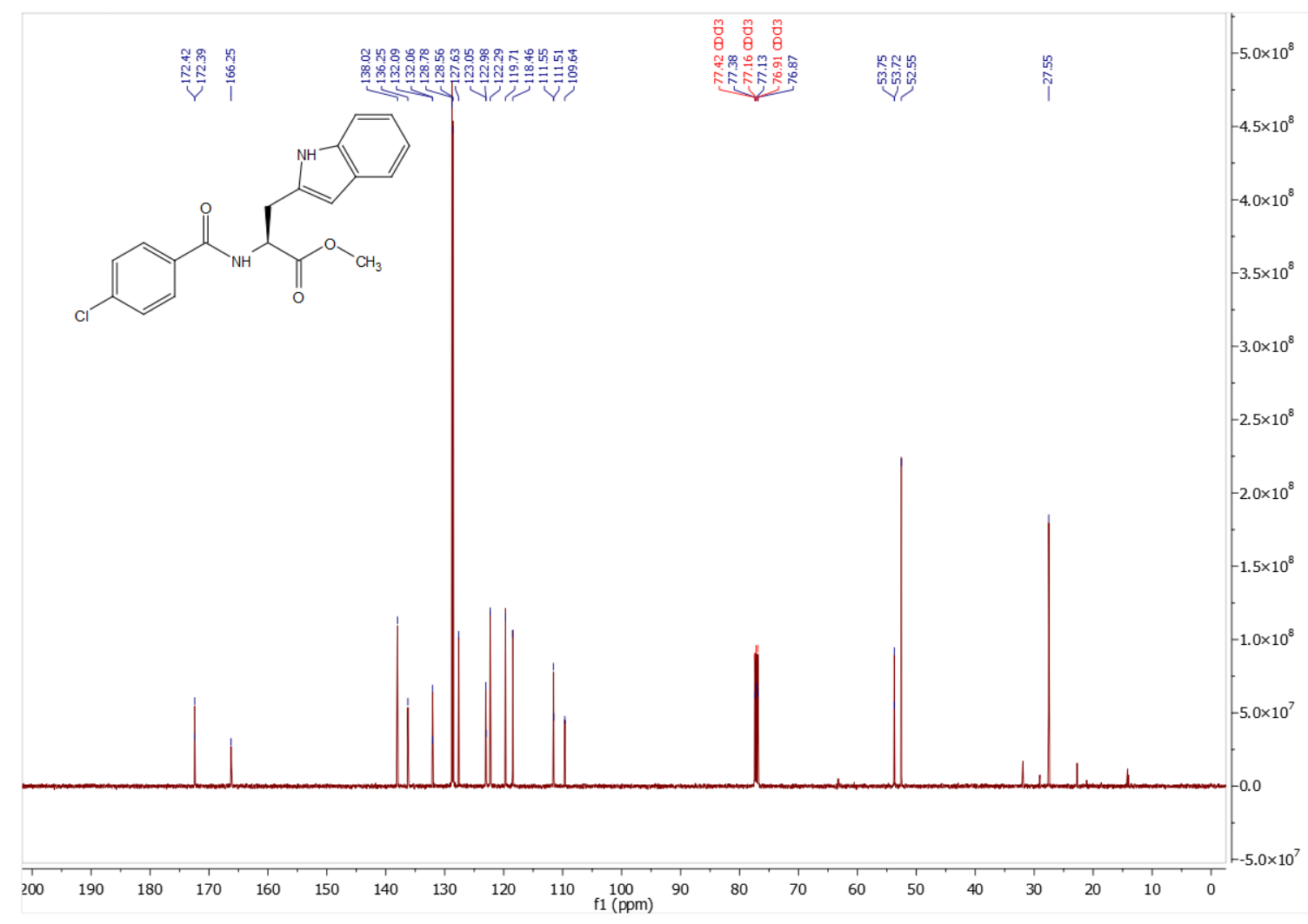

${ }^{13} \mathrm{C}$ NMR spectrum of compound $41\left(126 \mathrm{MHz}, \mathrm{CDCl}_{3}, \mathrm{rt}\right)$. 


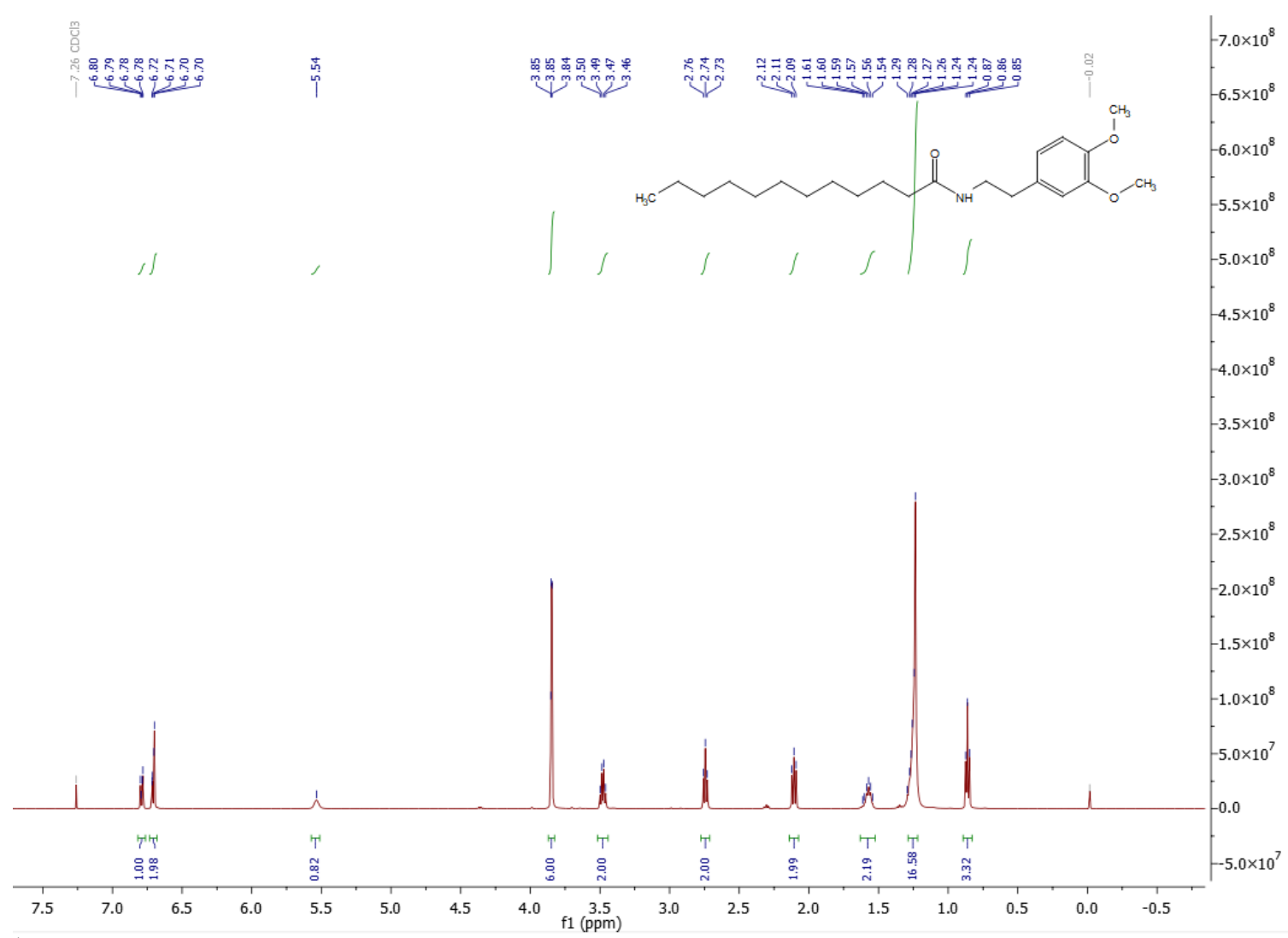

${ }^{1} \mathrm{H}$ NMR spectrum of compound $42\left(500 \mathrm{MHz}, \mathrm{CDCl}_{3}, \mathrm{rt}\right)$.

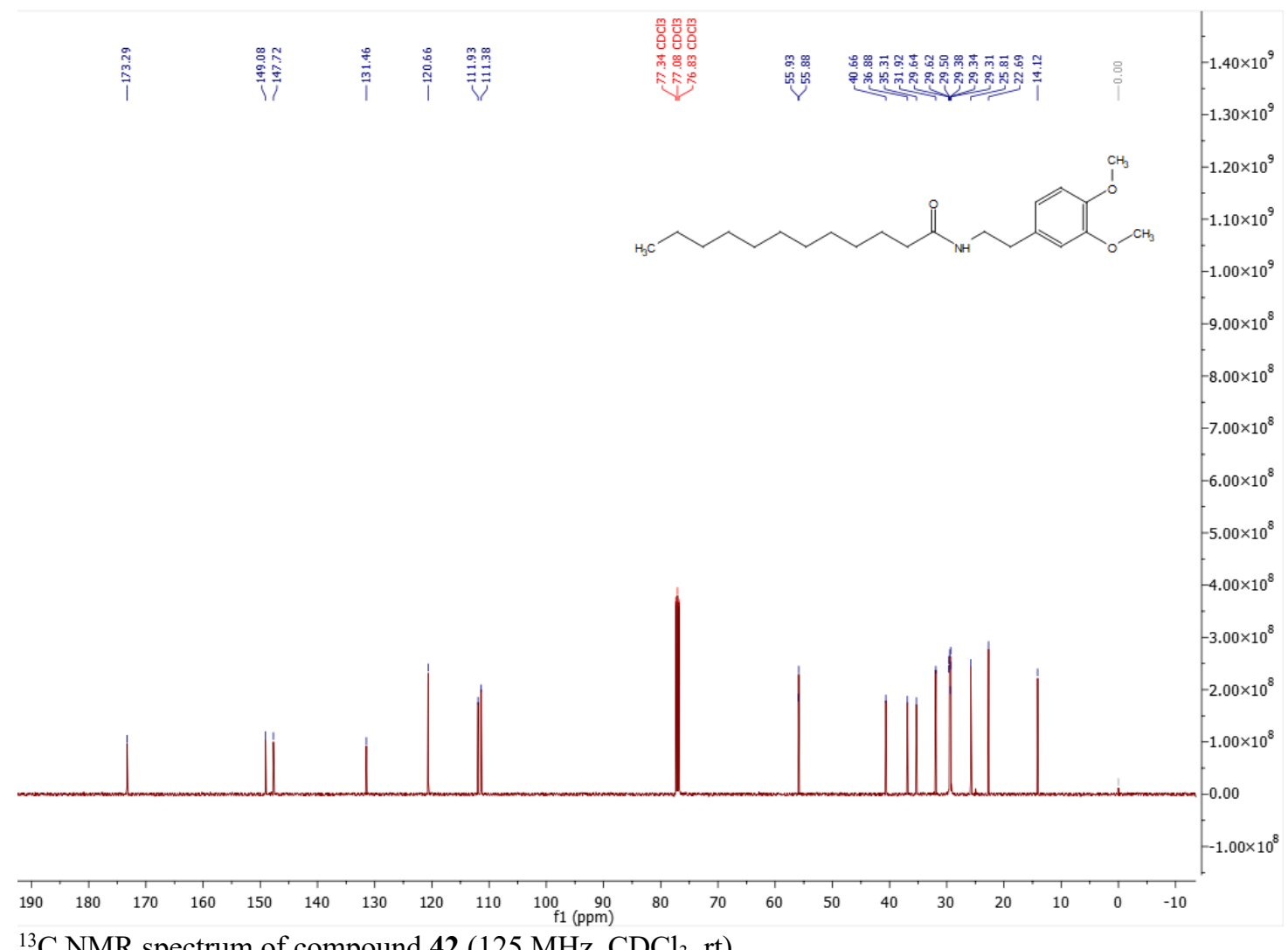

${ }^{13} \mathrm{C}$ NMR spectrum of compound $42\left(125 \mathrm{MHz}, \mathrm{CDCl}_{3}, \mathrm{rt}\right)$. 


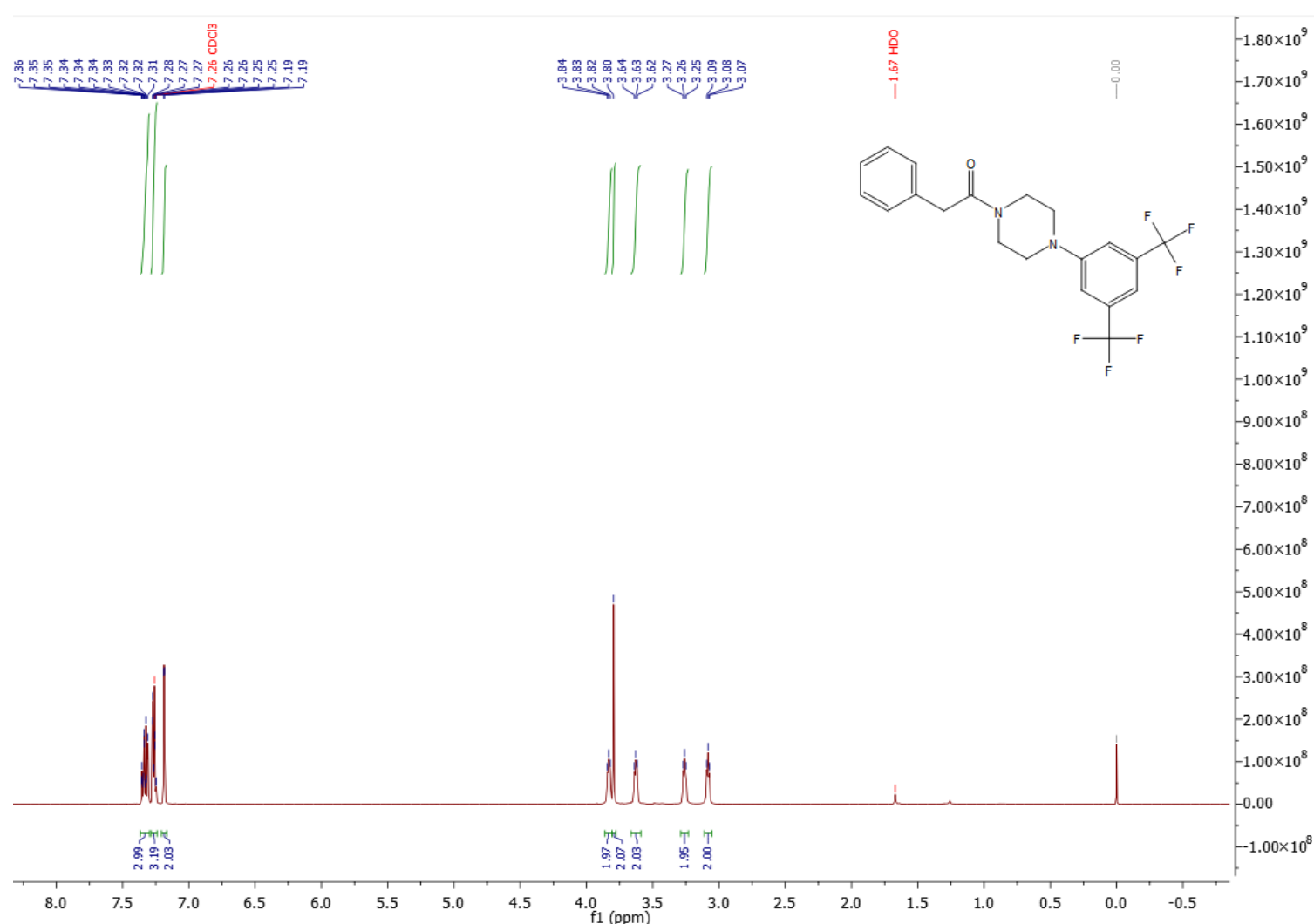

${ }^{1} \mathrm{H}$ NMR spectrum of compound $43\left(500 \mathrm{MHz}, \mathrm{CDCl}_{3}\right.$, rt).

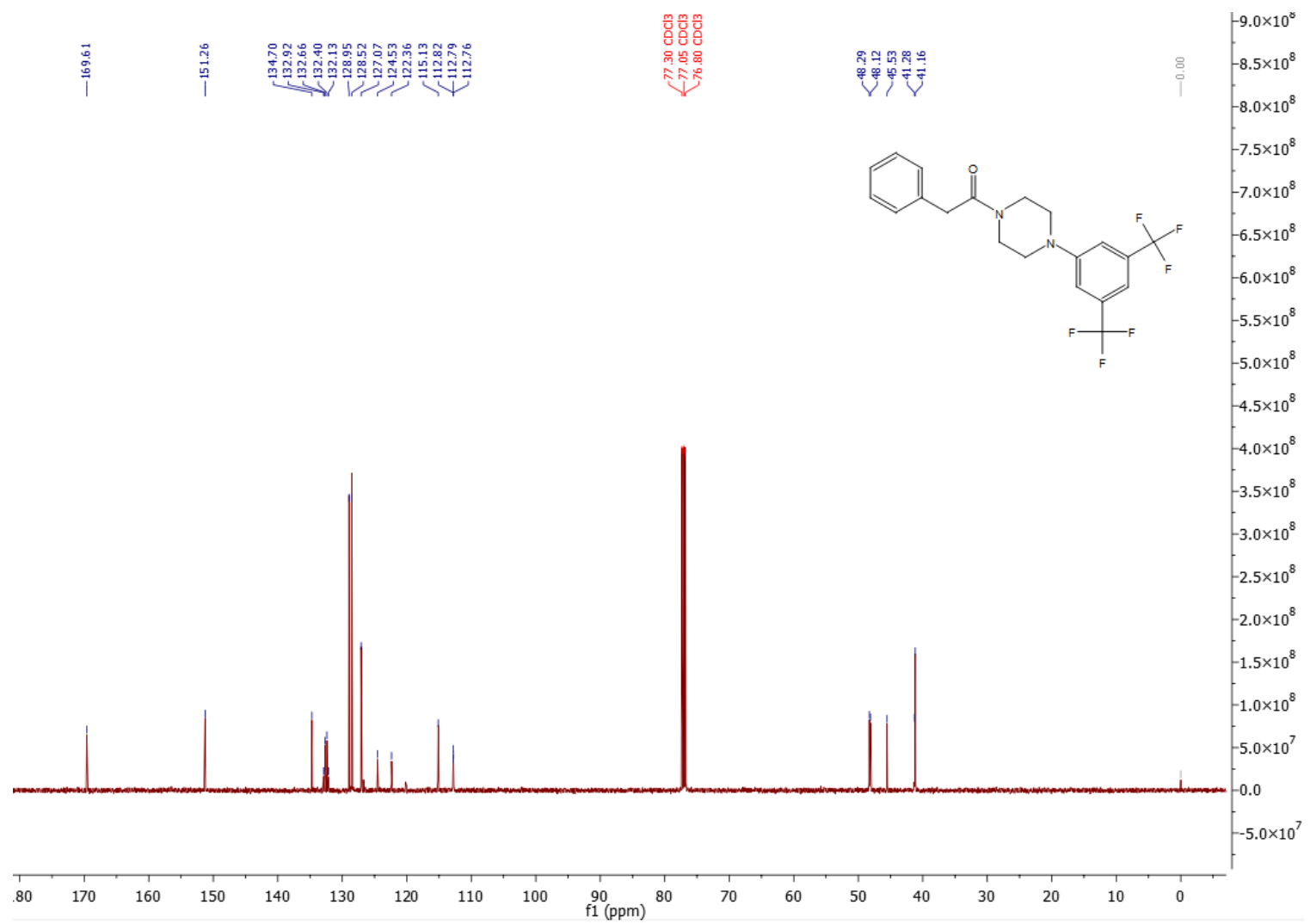

${ }^{13} \mathrm{C}$ NMR spectrum of compound $43\left(126 \mathrm{MHz}, \mathrm{CDCl}_{3}, \mathrm{rt}\right)$. 


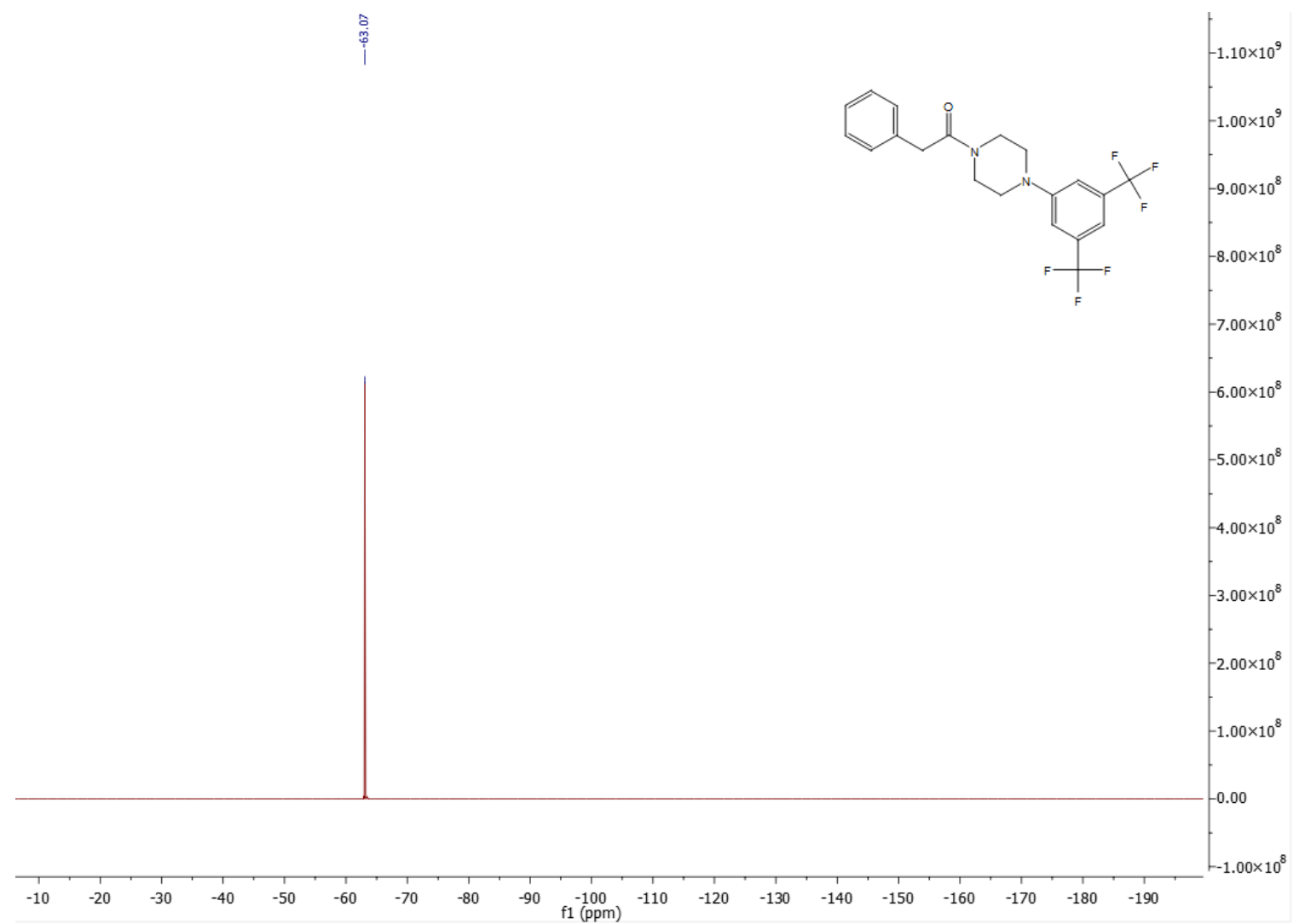

${ }^{19} \mathrm{~F}$ NMR spectrum of compound $43\left(471 \mathrm{MHz}, \mathrm{CDCl}_{3}, \mathrm{rt}\right)$. 


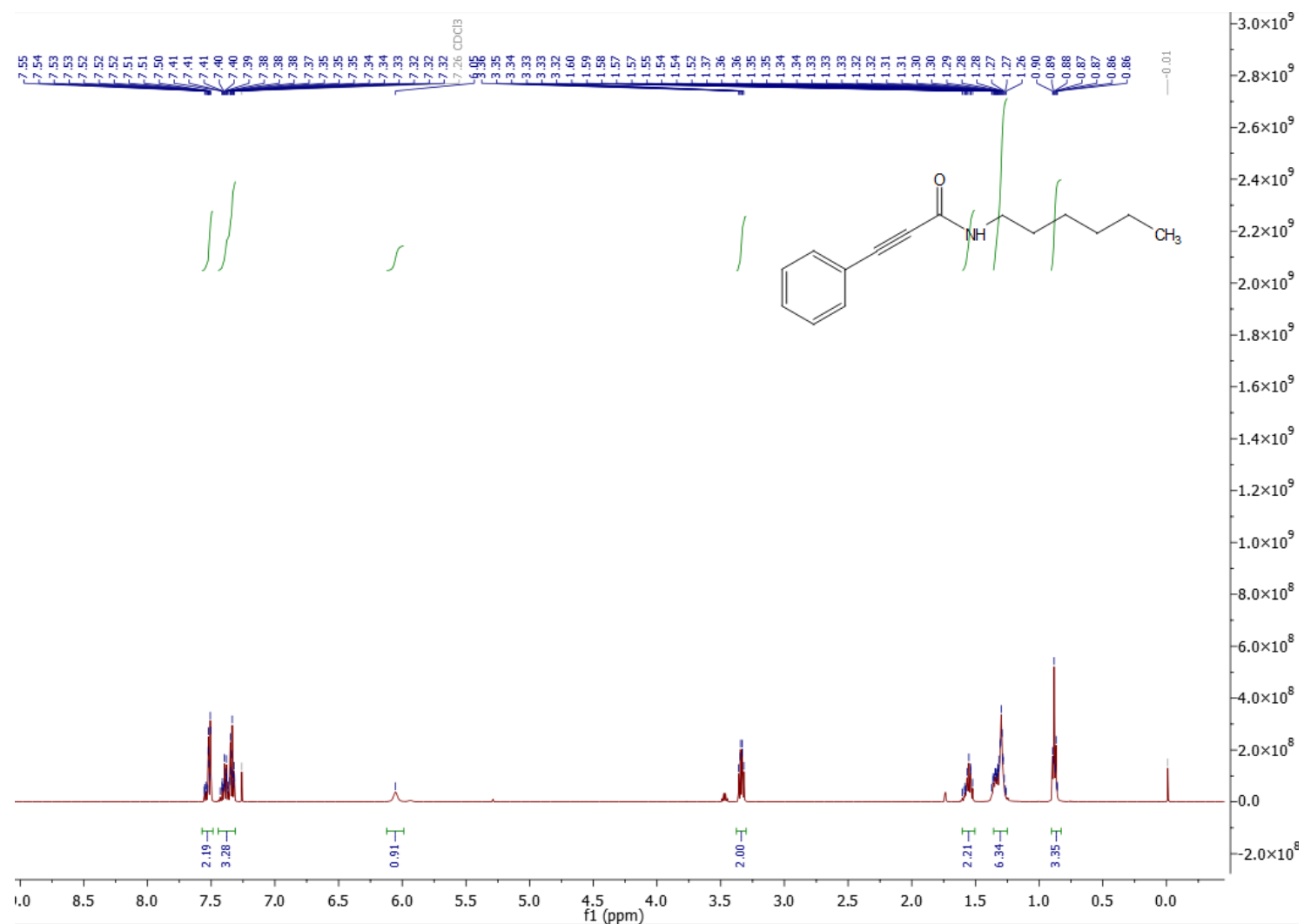

${ }^{1} \mathrm{H}$ NMR spectrum of compound $44\left(500 \mathrm{MHz}, \mathrm{CDCl}_{3}, \mathrm{rt}\right)$.

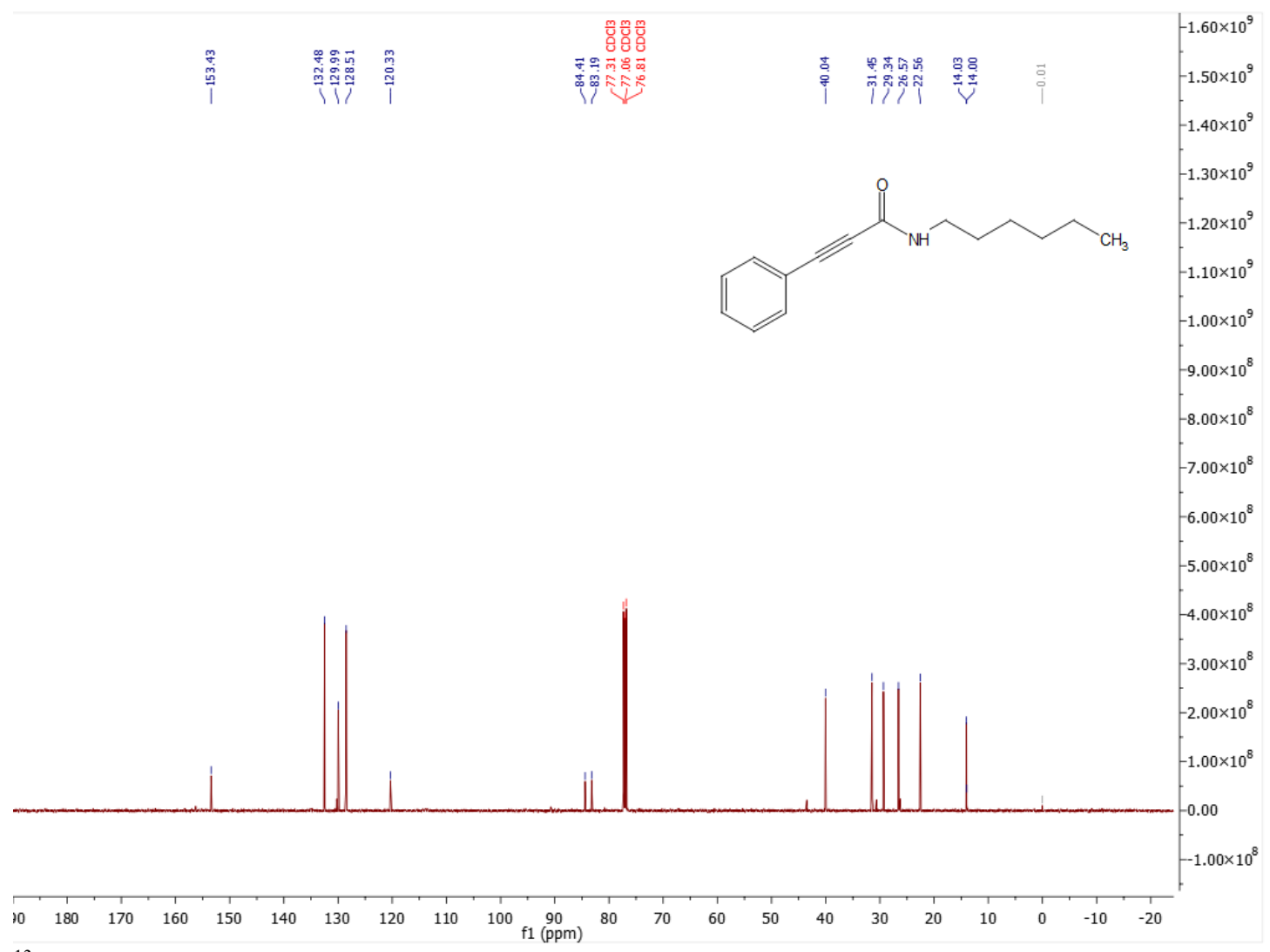

${ }^{13} \mathrm{C}$ NMR spectrum of compound $44\left(126 \mathrm{MHz}, \mathrm{CDCl}_{3}, \mathrm{rt}\right)$. 


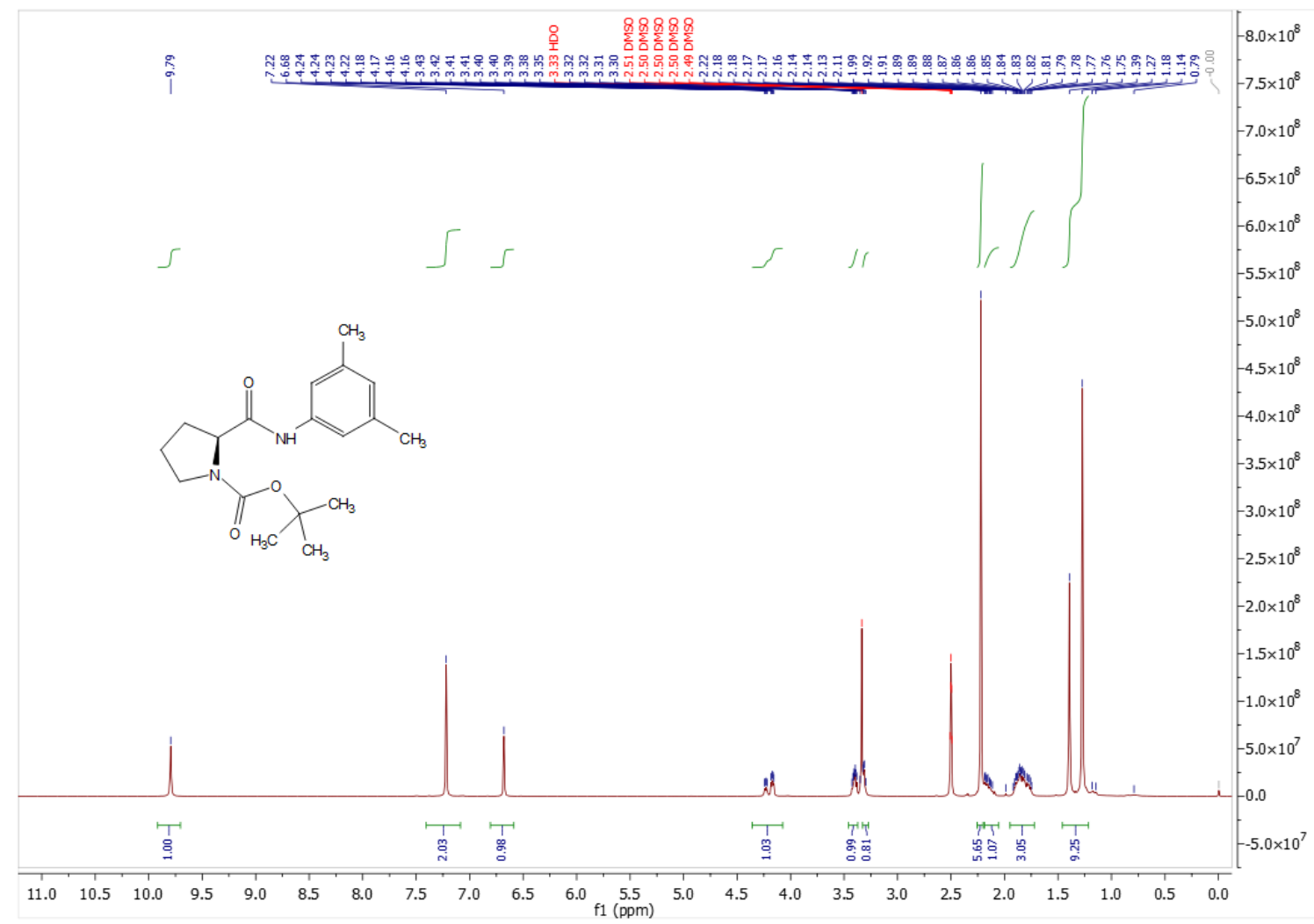

${ }^{1} \mathrm{H}$ NMR spectrum of compound 45 (500 MHz, DMSO- $d 6$, rt).

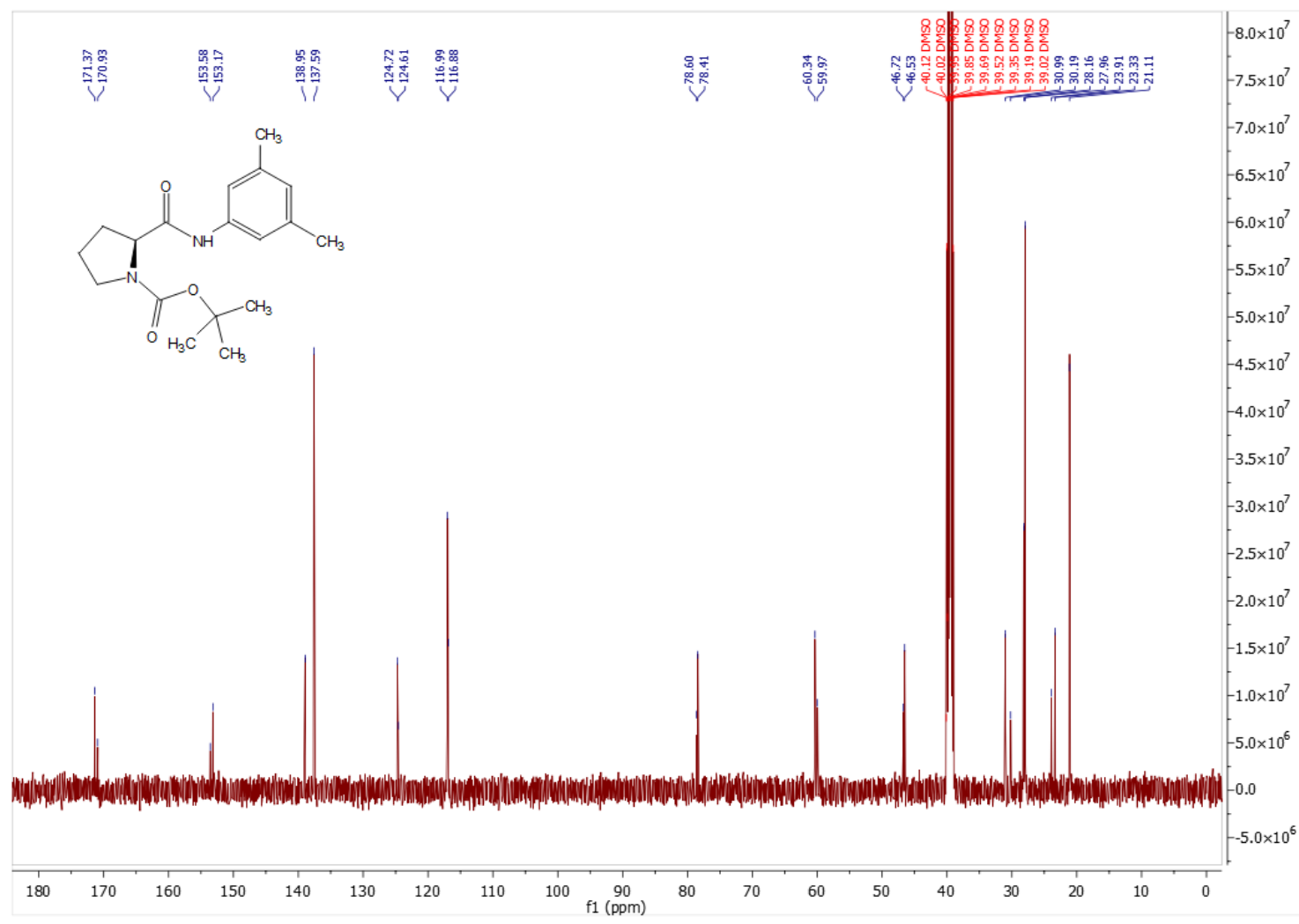

${ }^{13} \mathrm{C}$ NMR spectrum of compound 45 (126 MHz, DMSO- $d_{6}$, rt). 


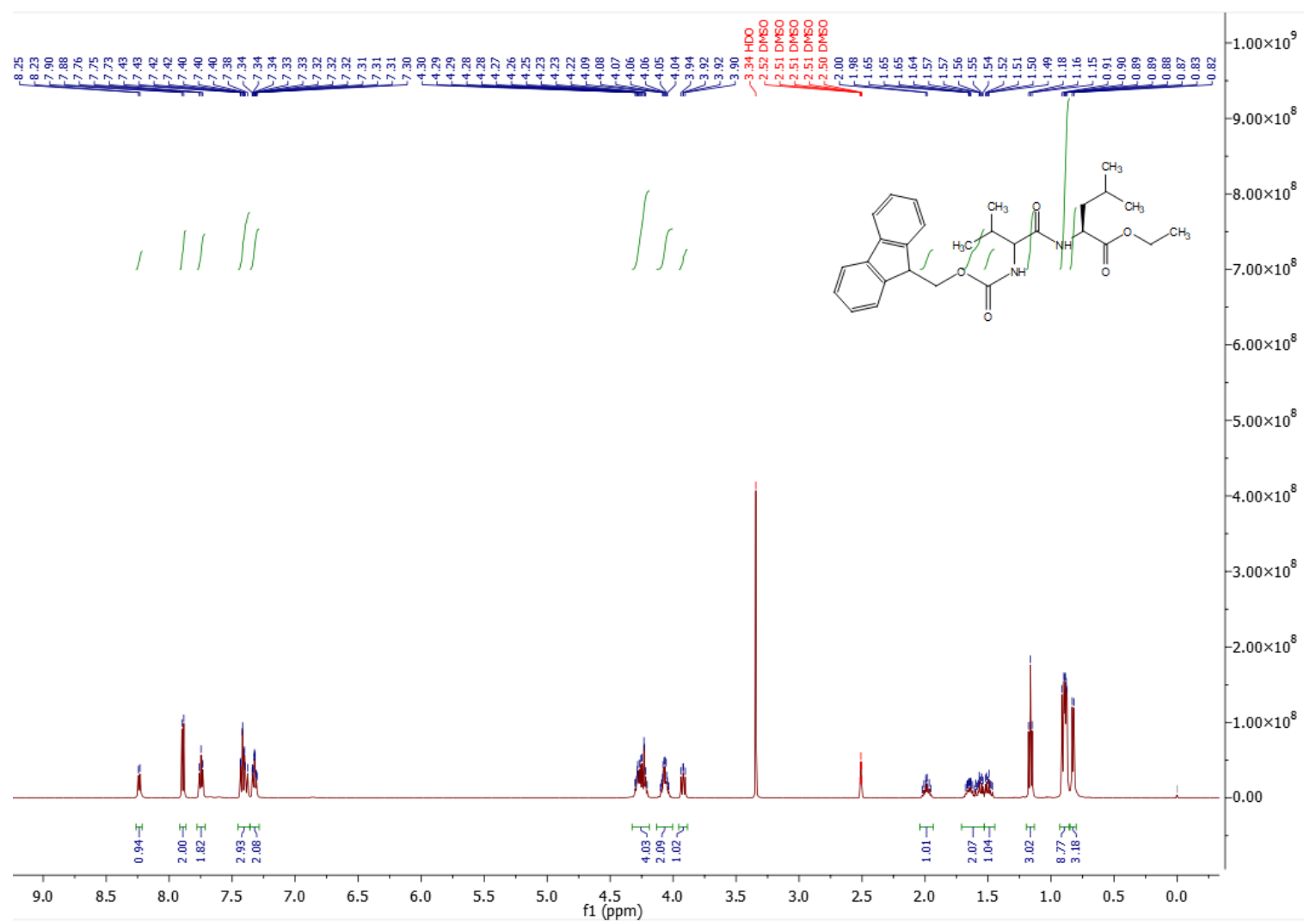

${ }^{1} \mathrm{H}$ NMR spectrum of compound 46 (500 MHz, DMSO- $d_{6}$, rt).

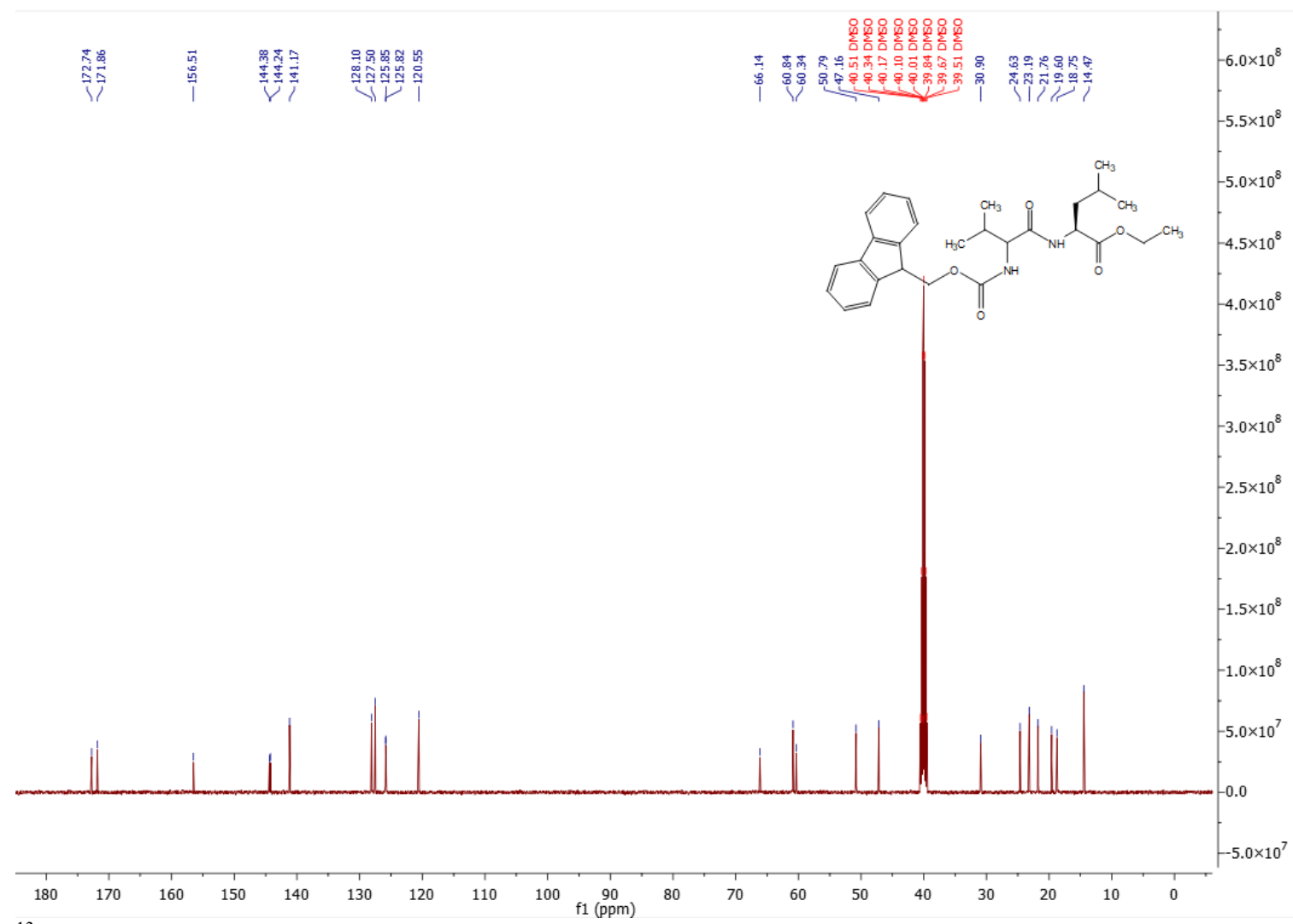

${ }^{13} \mathrm{C}$ NMR spectrum of compound 46 (126 MHz, DMSO- $d_{6}$, rt). 


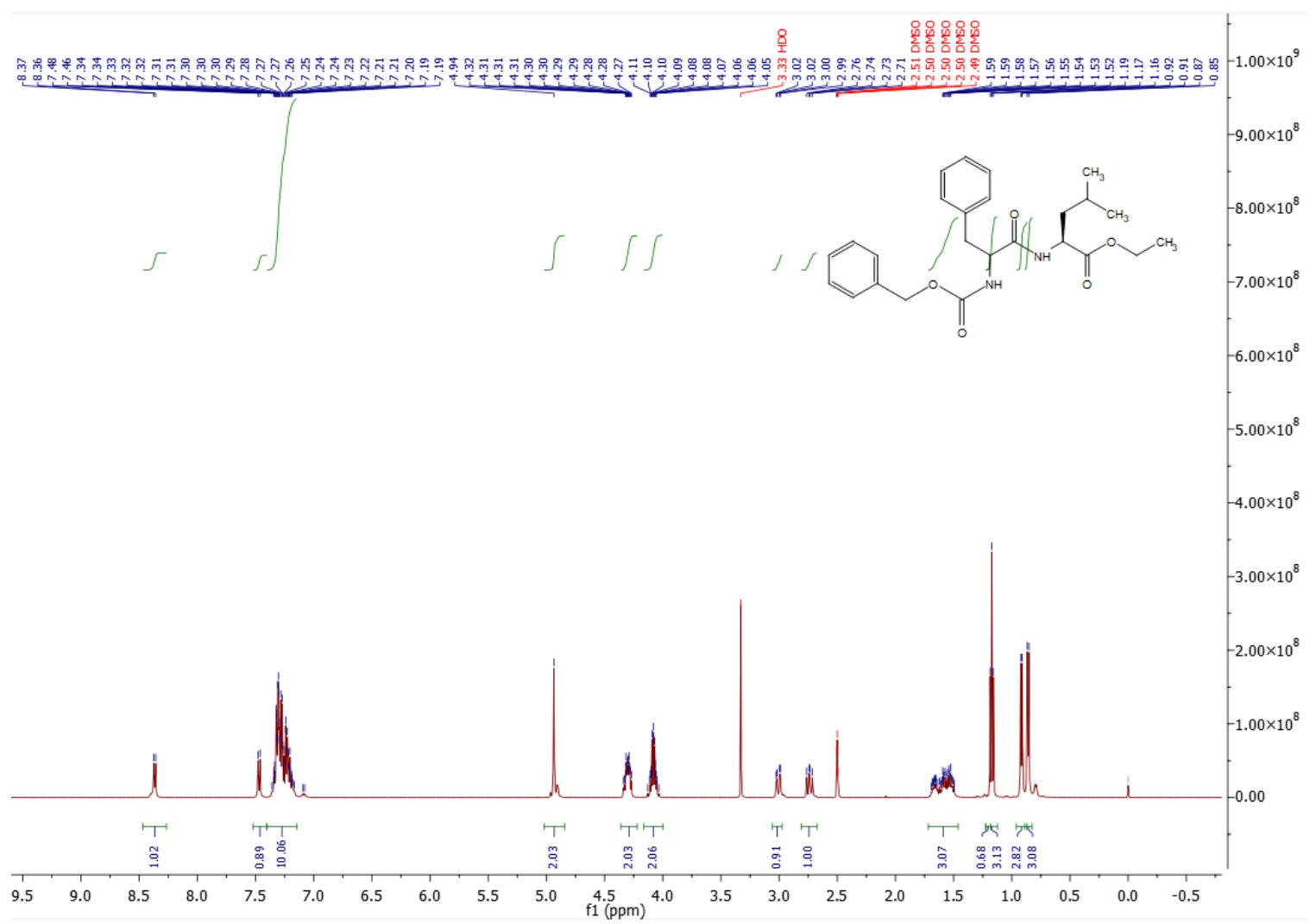

${ }^{1} \mathrm{H}$ NMR spectrum of compound 47 (500 MHz, DMSO- $d_{6}, \mathrm{rt}$ ).

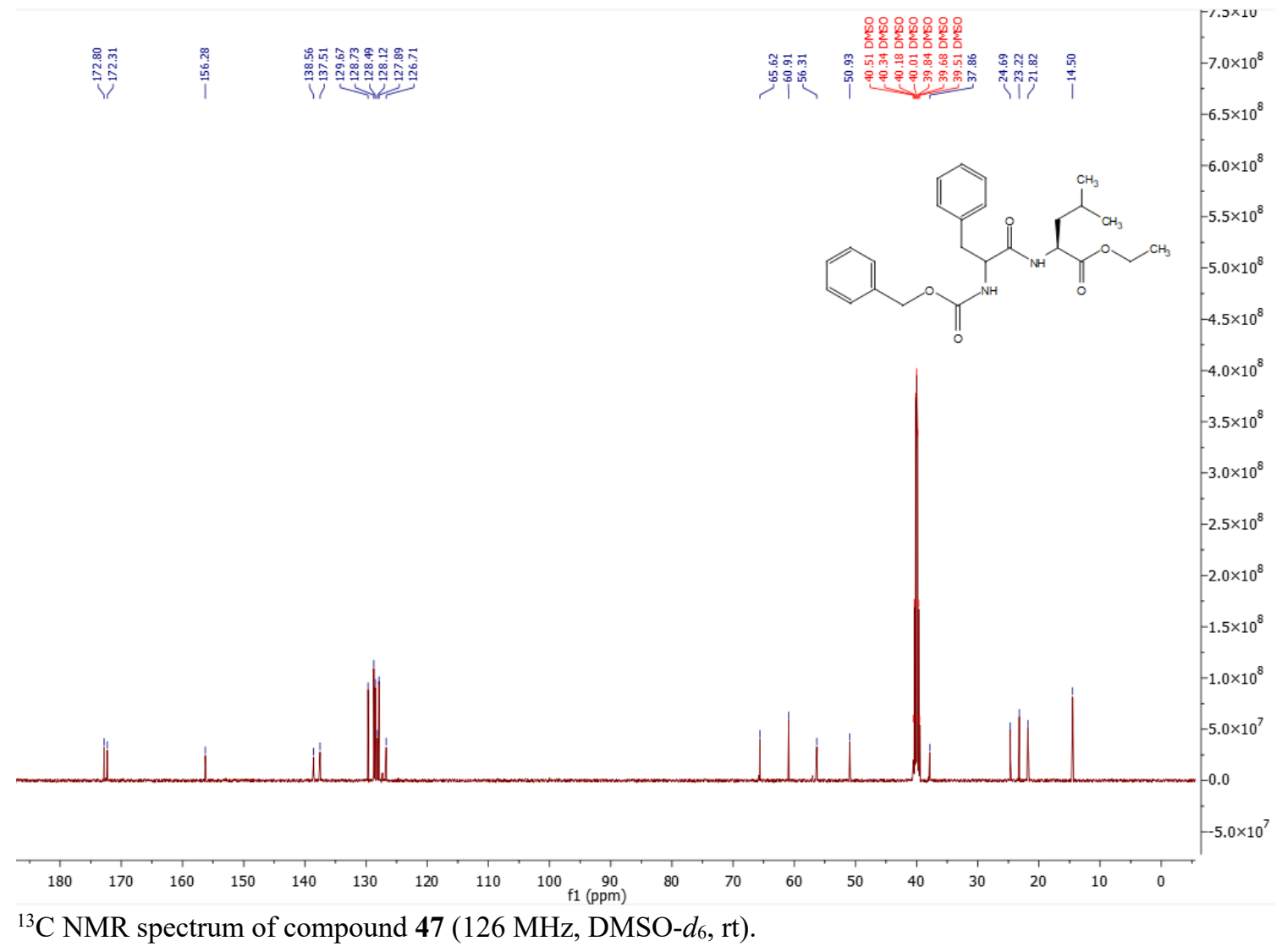




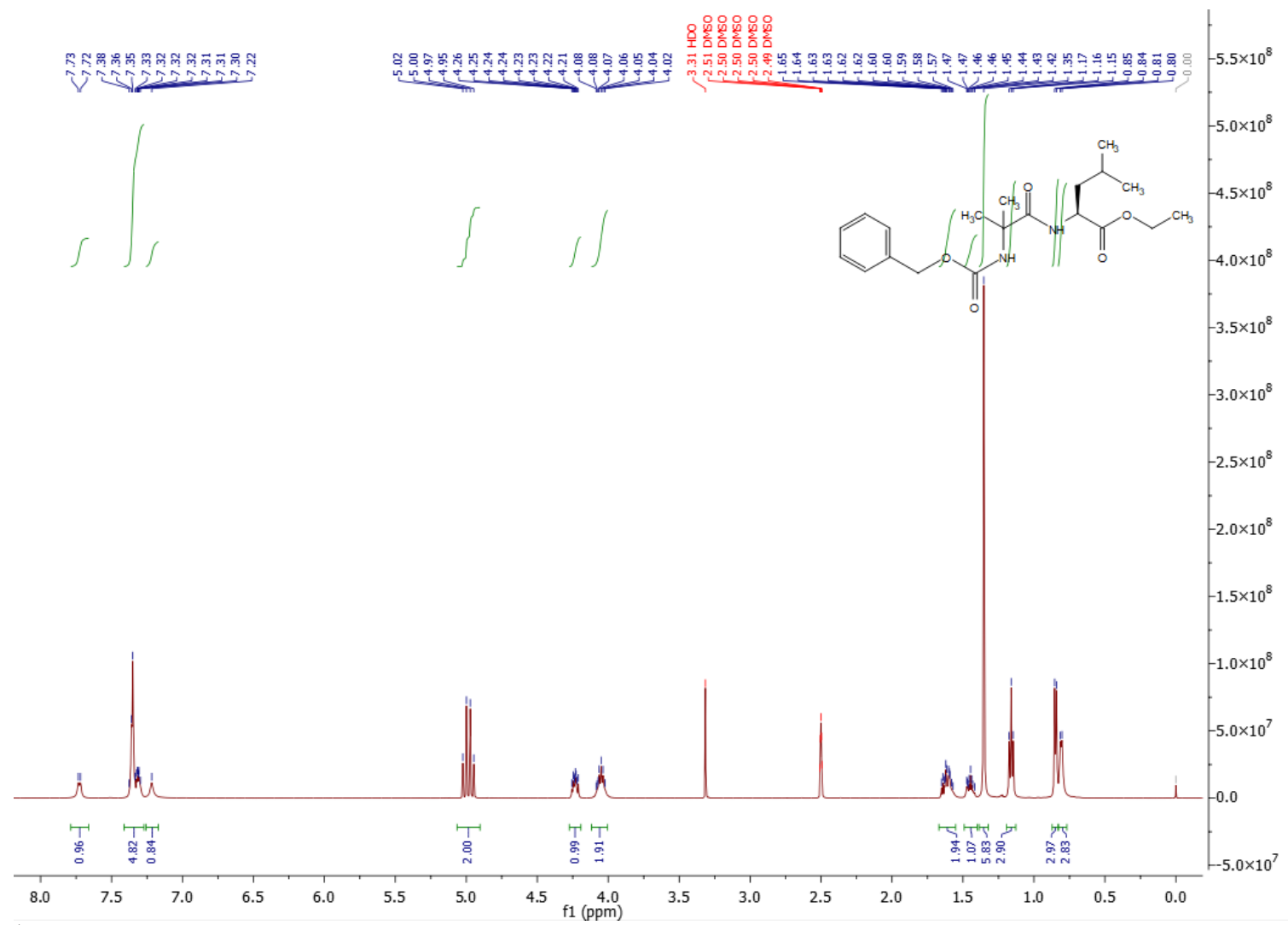

${ }^{1} \mathrm{H}$ NMR spectrum of compound 48 (500 MHz, DMSO- $\left.d_{6}, \mathrm{rt}\right)$.

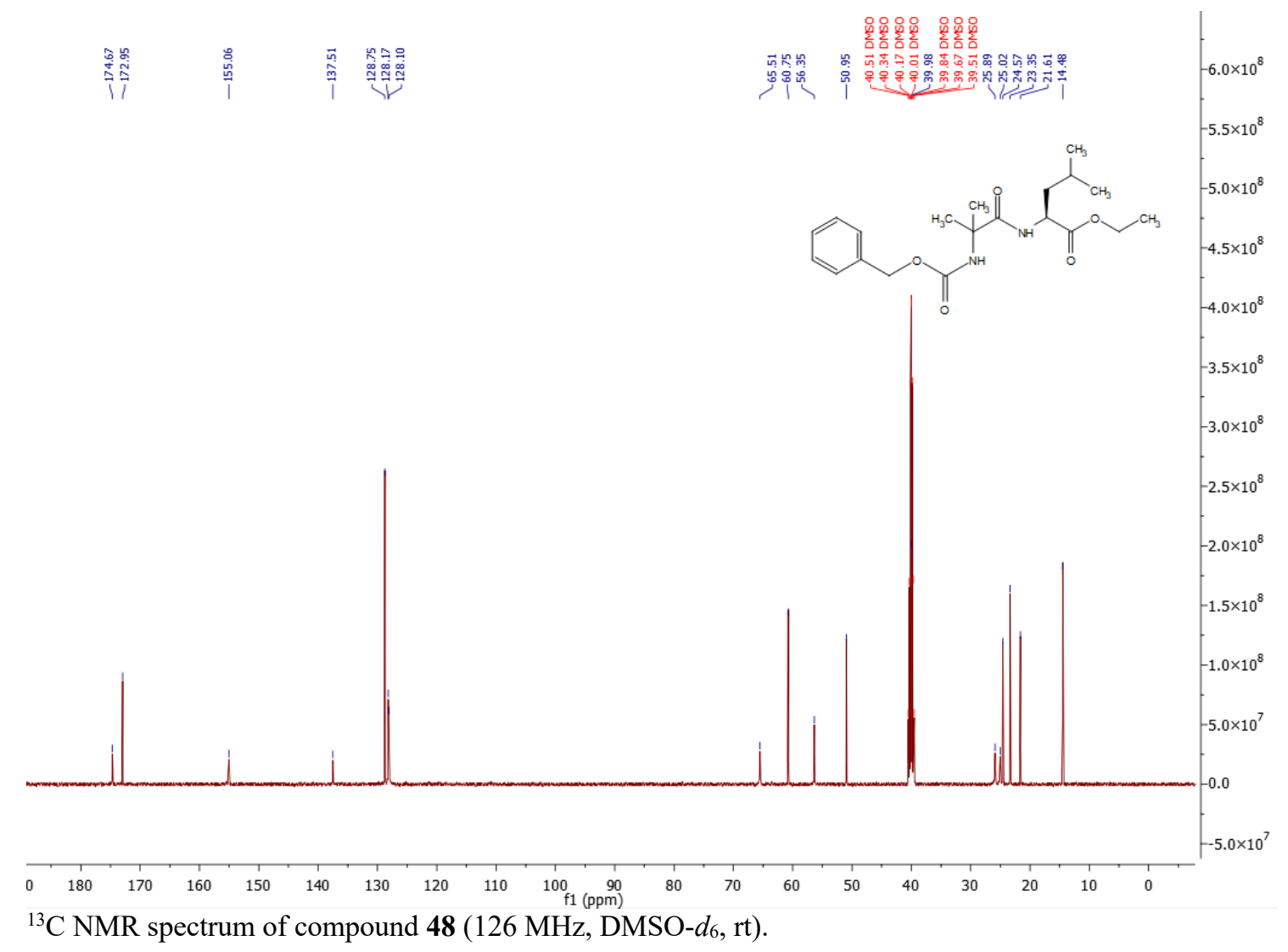




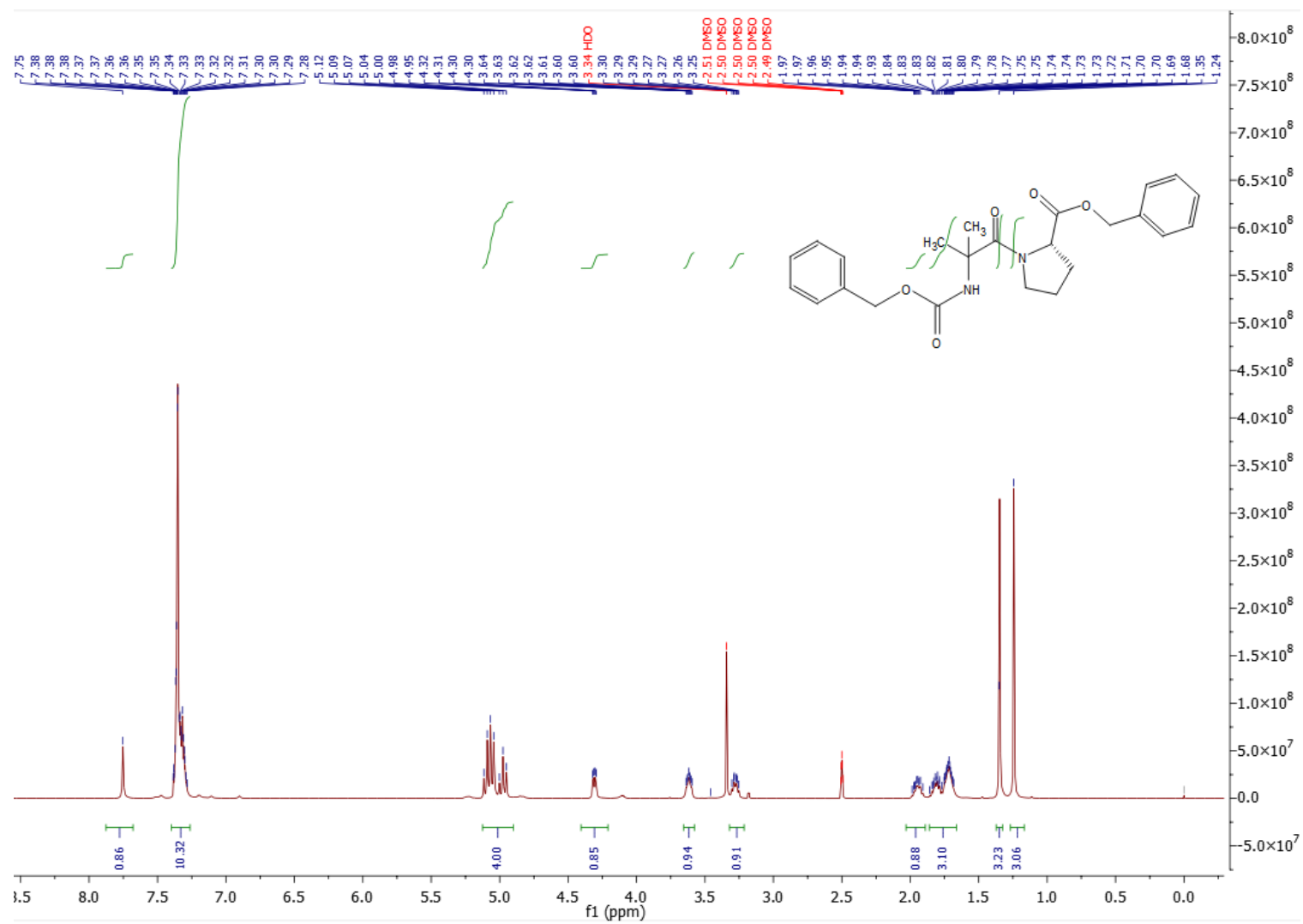

${ }^{1} \mathrm{H}$ NMR spectrum of compound 49 (500 MHz, DMSO- $\left.d_{6}, \mathrm{rt}\right)$.

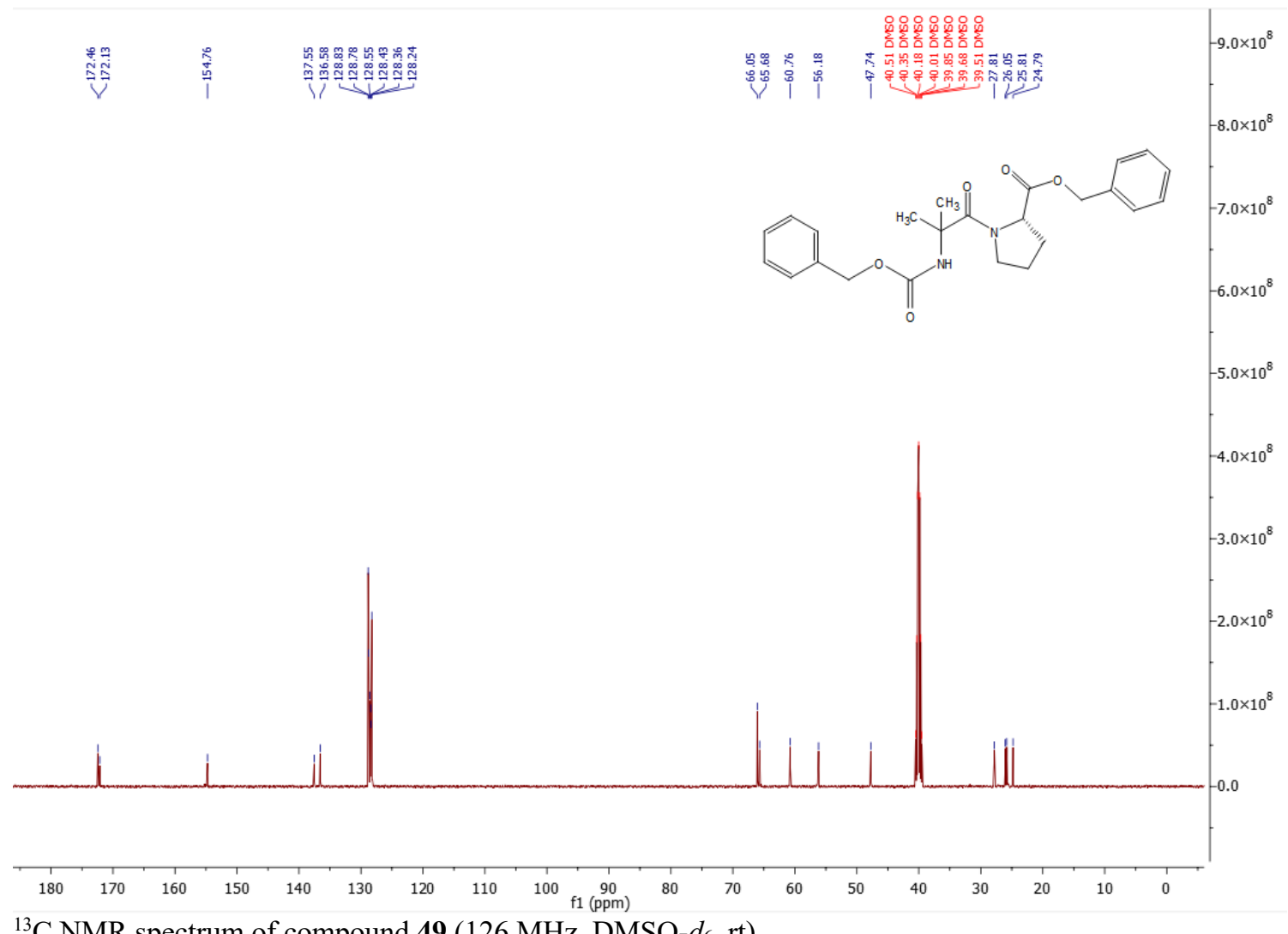

${ }^{13} \mathrm{C}$ NMR spectrum of compound 49 (126 MHz, DMSO- $d_{6}$, rt). 


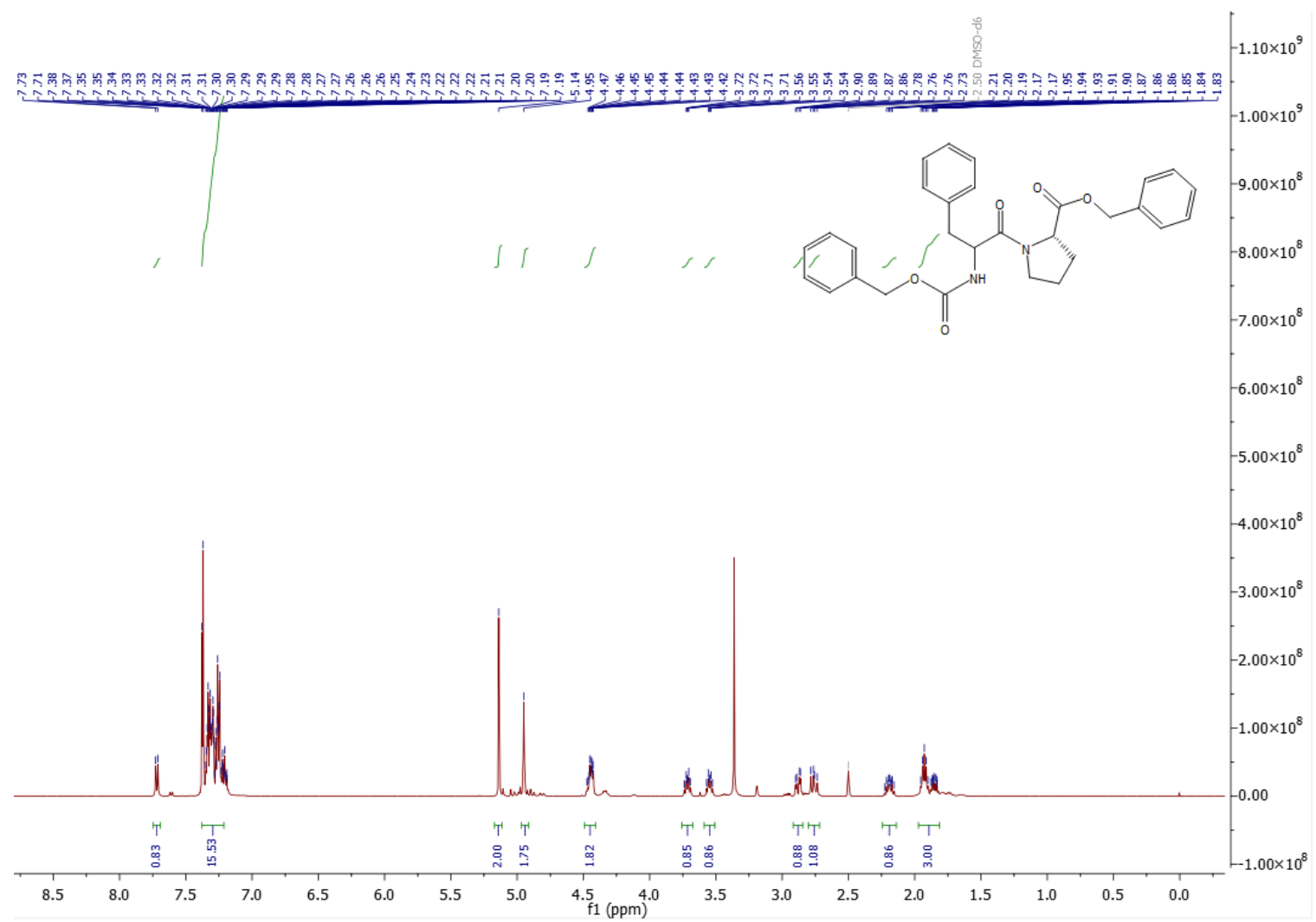

${ }^{1} \mathrm{H}$ NMR spectrum of compound $\mathbf{5 0}$ (500 MHz, DMSO- $\left.d_{6}, \mathrm{rt}\right)$.

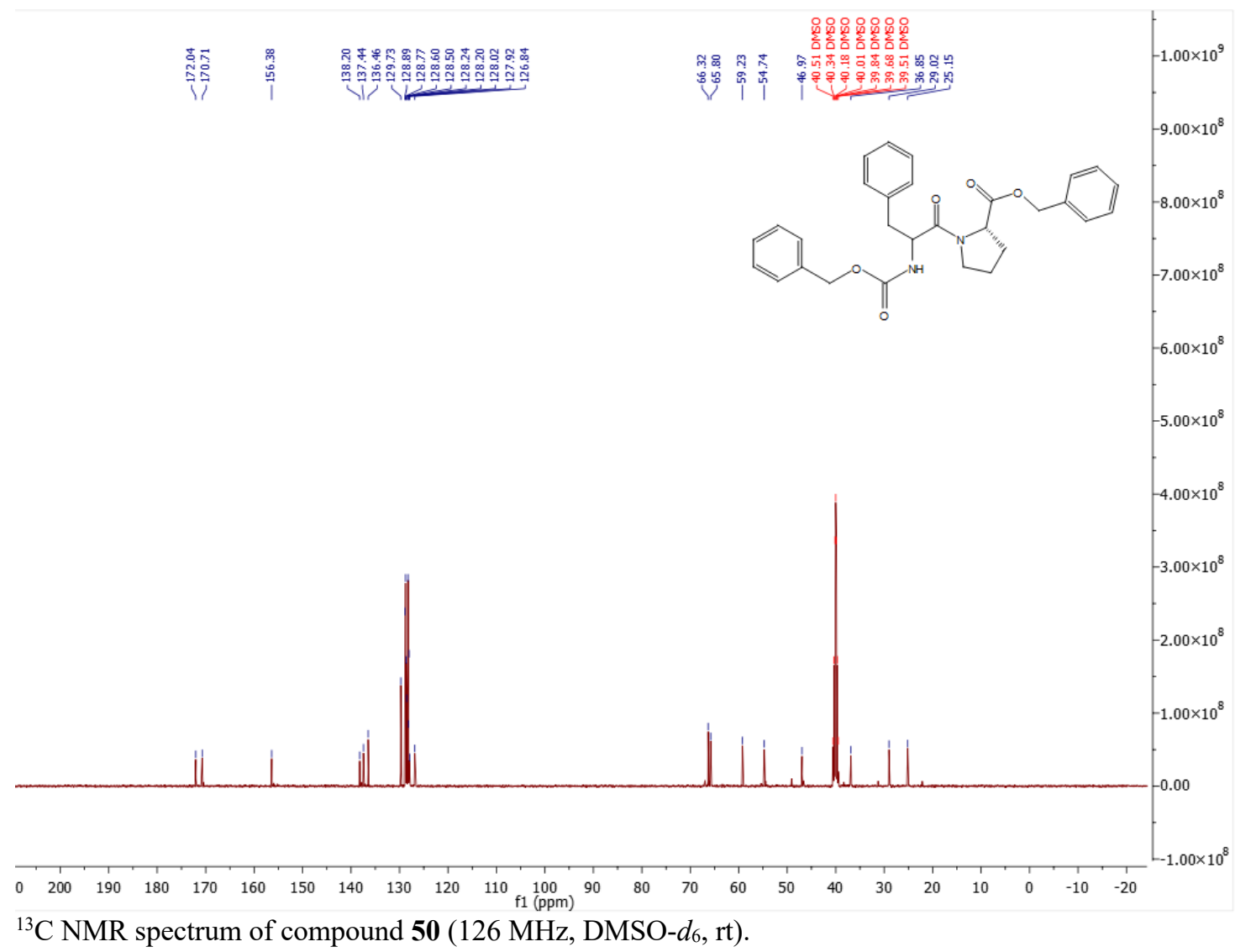




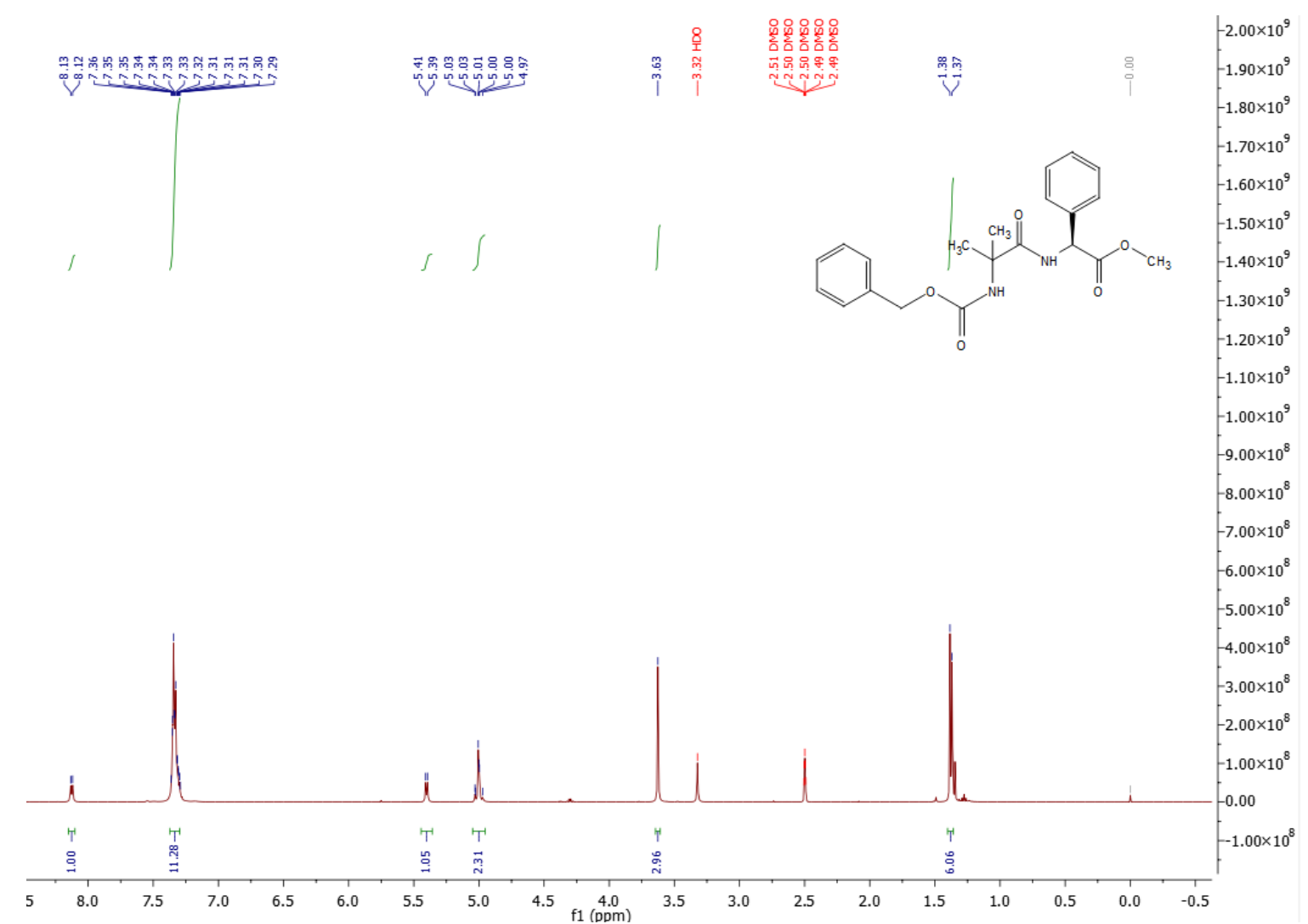

${ }^{1} \mathrm{H}$ NMR spectrum of compound 51a (500 MHz, DMSO- $d_{6}$, rt).

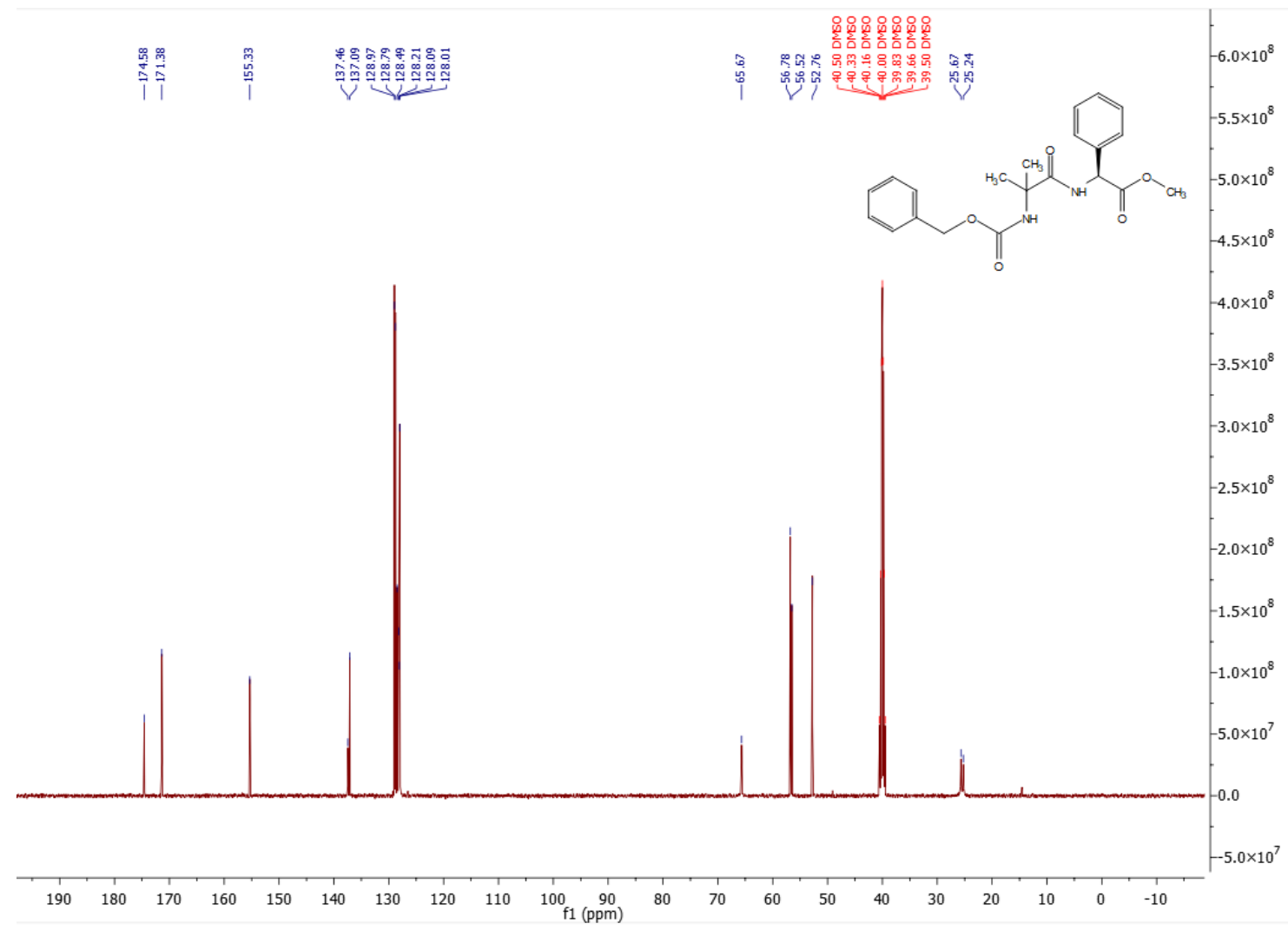

${ }^{13} \mathrm{C}$ NMR spectrum of compound 51a (126 MHz, DMSO- $\left.d 6, \mathrm{rt}\right)$. 


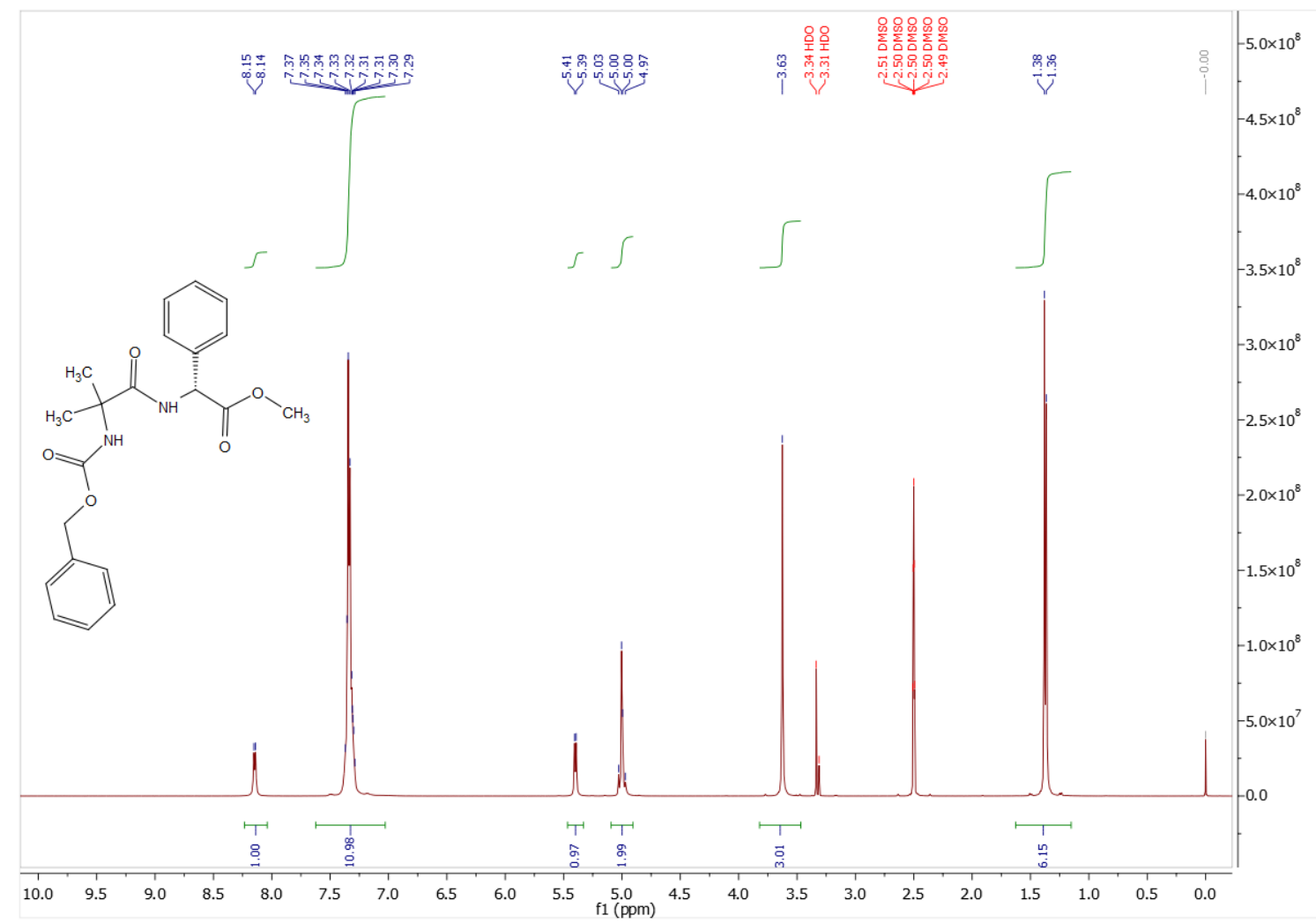

${ }^{1} \mathrm{H}$ NMR spectrum of compound $\mathbf{5 1 b}\left(500 \mathrm{MHz}\right.$, DMSO- $d_{6}$, rt).

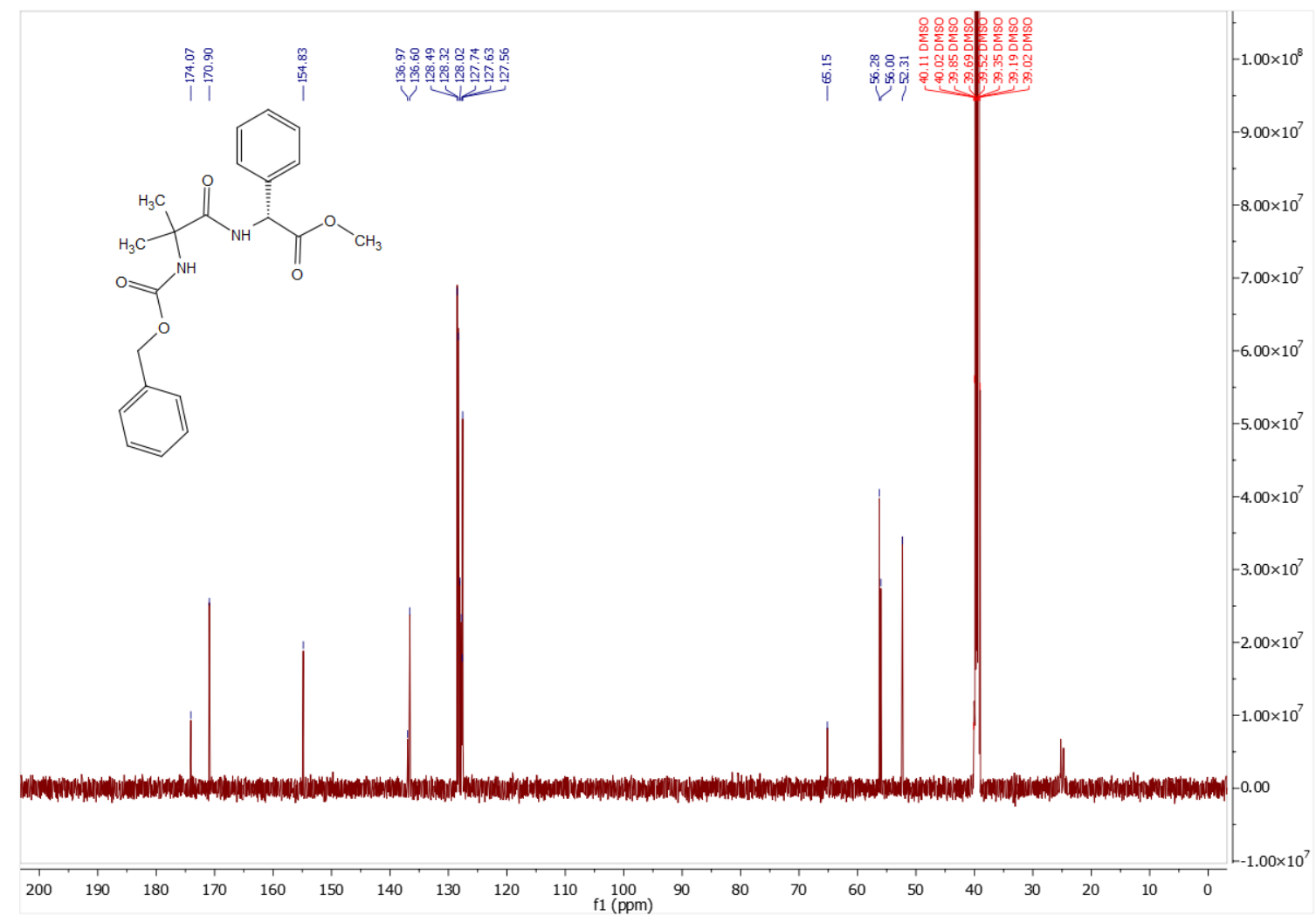

${ }^{13} \mathrm{C}$ NMR spectrum of compound $\mathbf{5 1 b}$ (126 MHz, DMSO- $d_{6}, \mathrm{rt}$ ). 


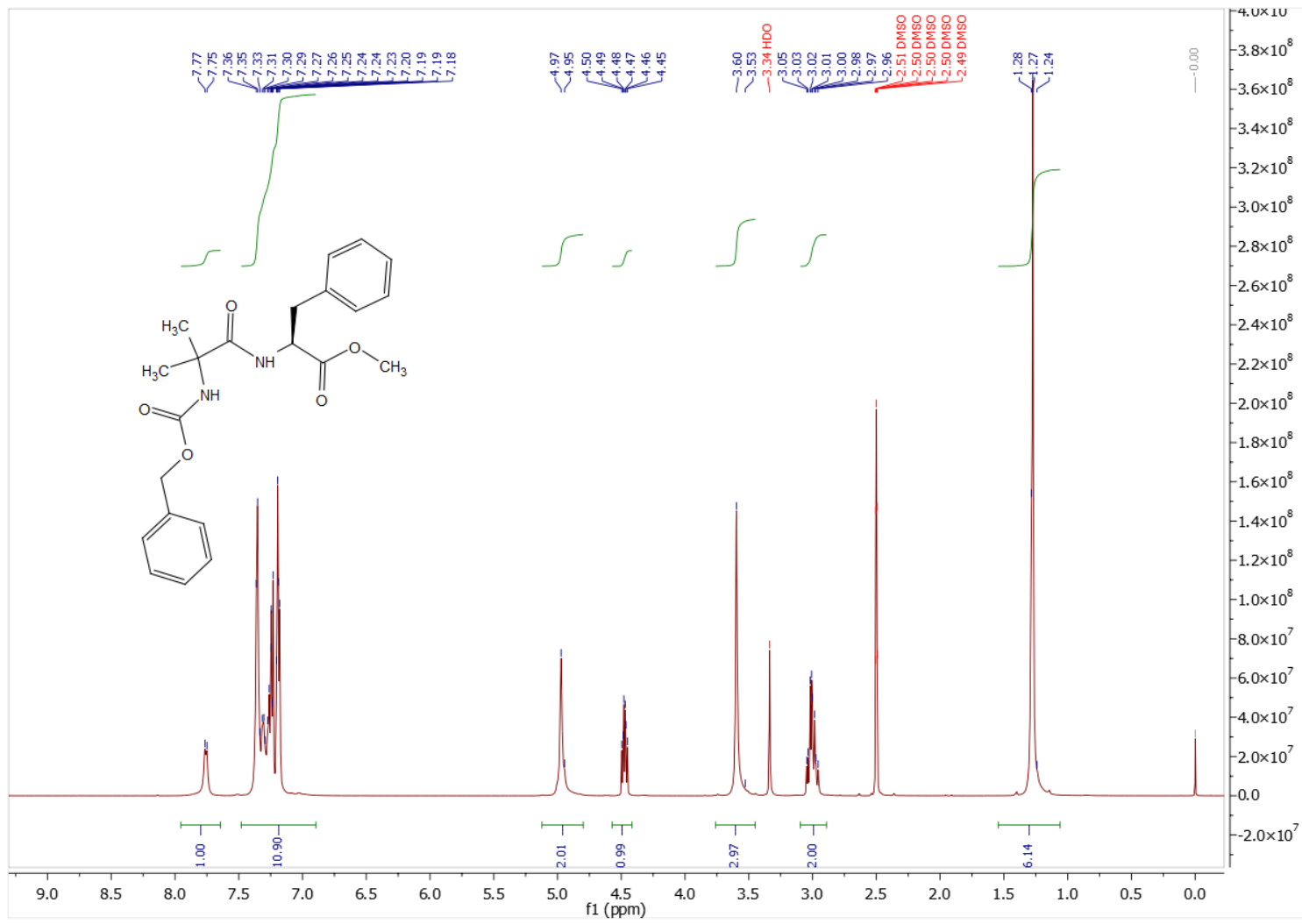

${ }^{1} \mathrm{H}$ NMR spectrum of compound 52a (500 MHz, DMSO- $d_{6}$, rt).

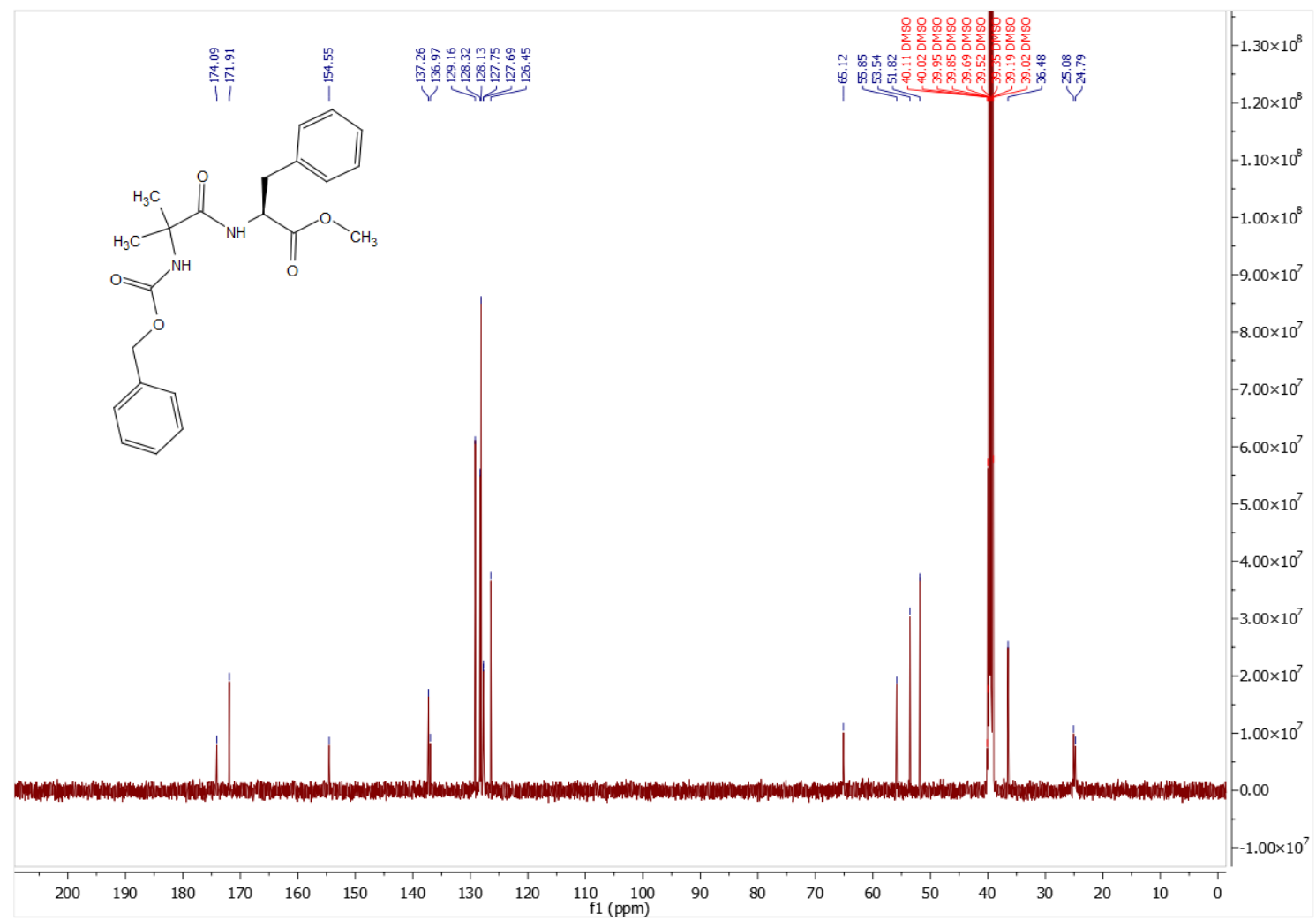

${ }^{13} \mathrm{C}$ NMR spectrum of compound 52a (126 MHz, DMSO- $\left.d_{6}, \mathrm{rt}\right)$. 


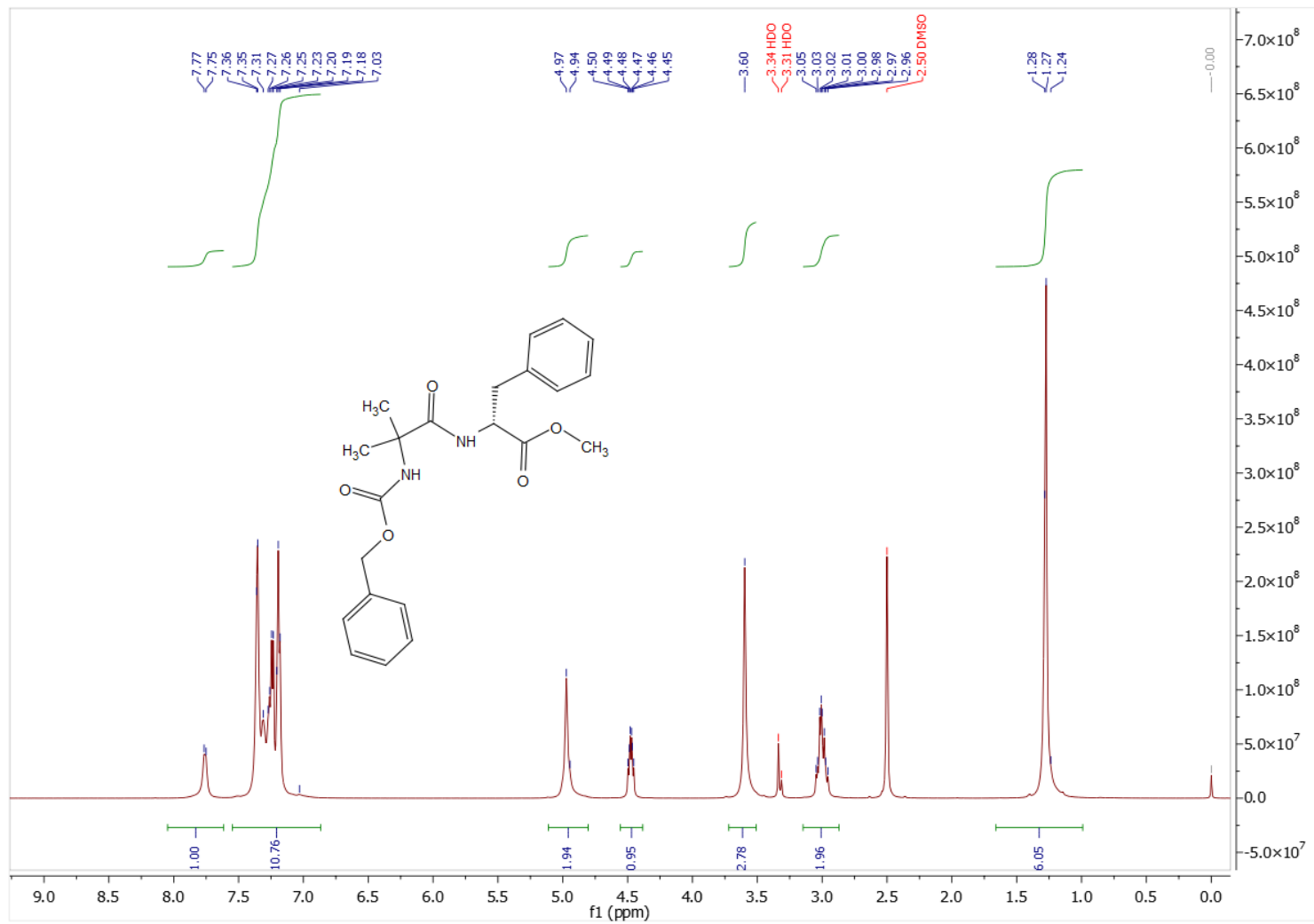

${ }^{1} \mathrm{H}$ NMR spectrum of compound 52b (500 MHz, DMSO- $d_{6}, \mathrm{rt}$ ).

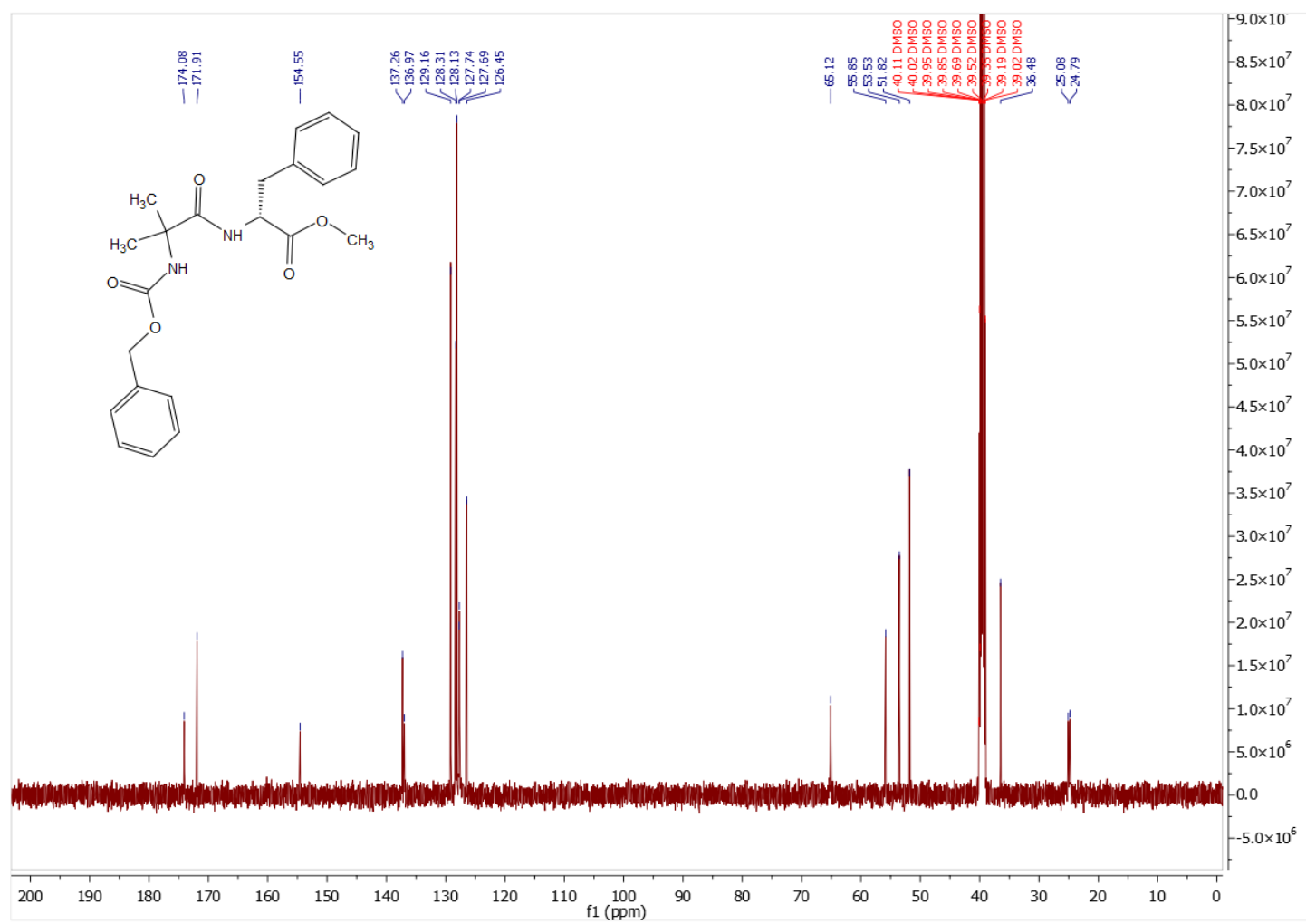

${ }^{13} \mathrm{C}$ NMR spectrum of compound 52b (126 MHz, DMSO- $\left.d_{6}, \mathrm{rt}\right)$. 


\section{Spectral Data Four Step One-Pot Product}
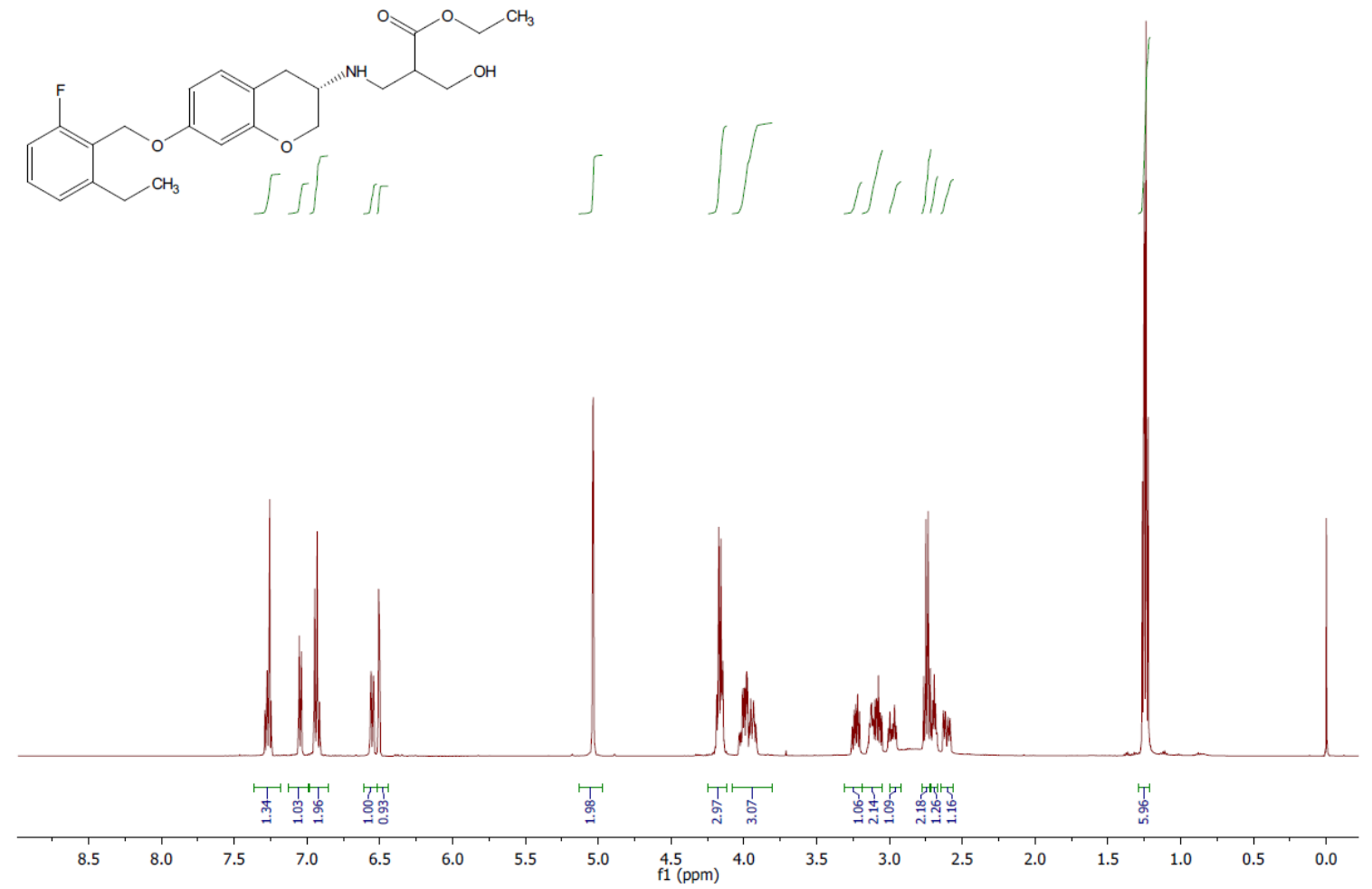

${ }^{1} \mathrm{H}$ NMR spectrum of compound 57 (500 MHz, $\mathrm{CDCl}_{3}, \mathrm{rt}$ ).
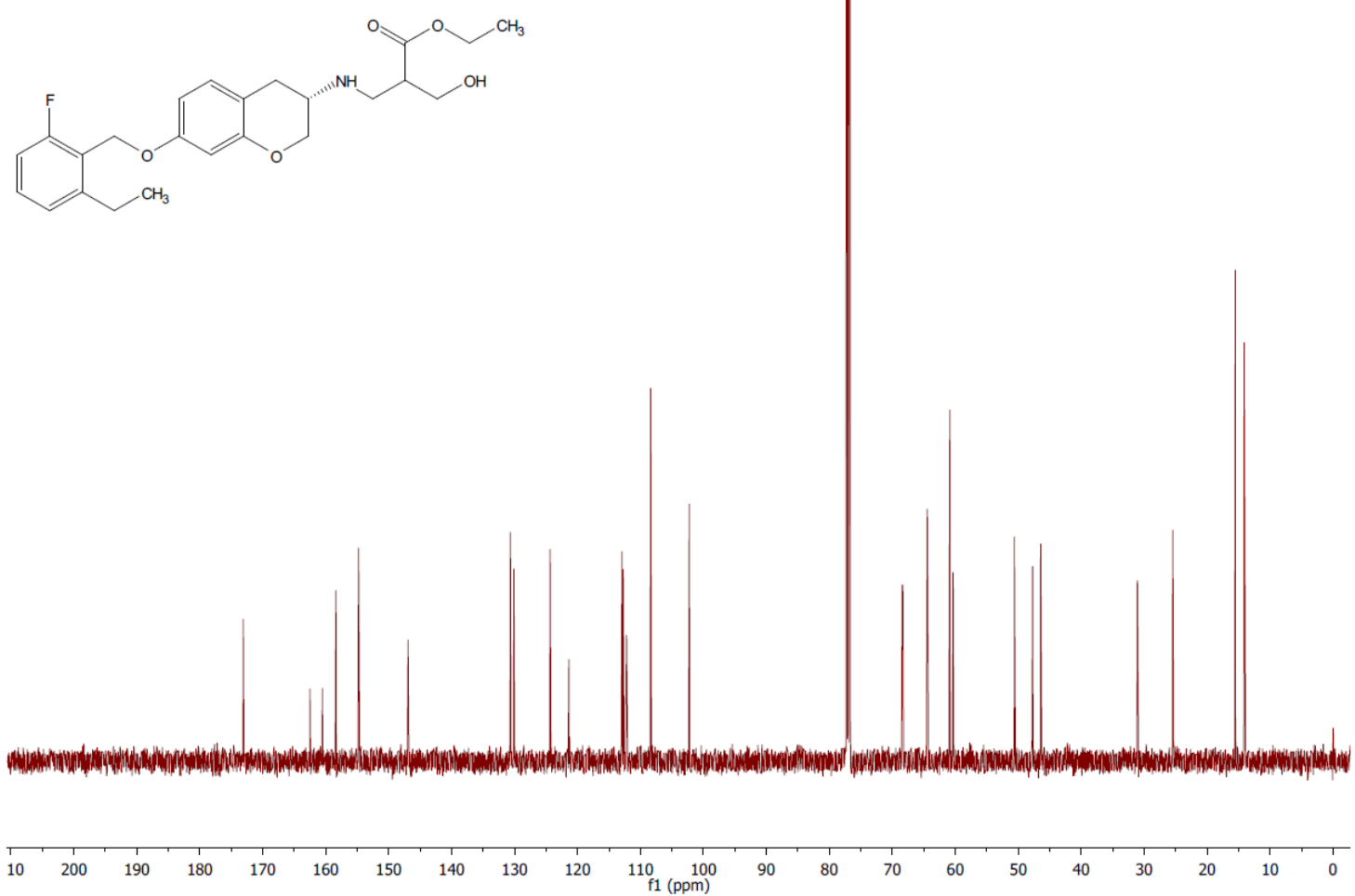

${ }^{13} \mathrm{C}$ NMR spectrum of compound $57\left(126 \mathrm{MHz}, \mathrm{CDCl}_{3}, \mathrm{rt}\right)$. 


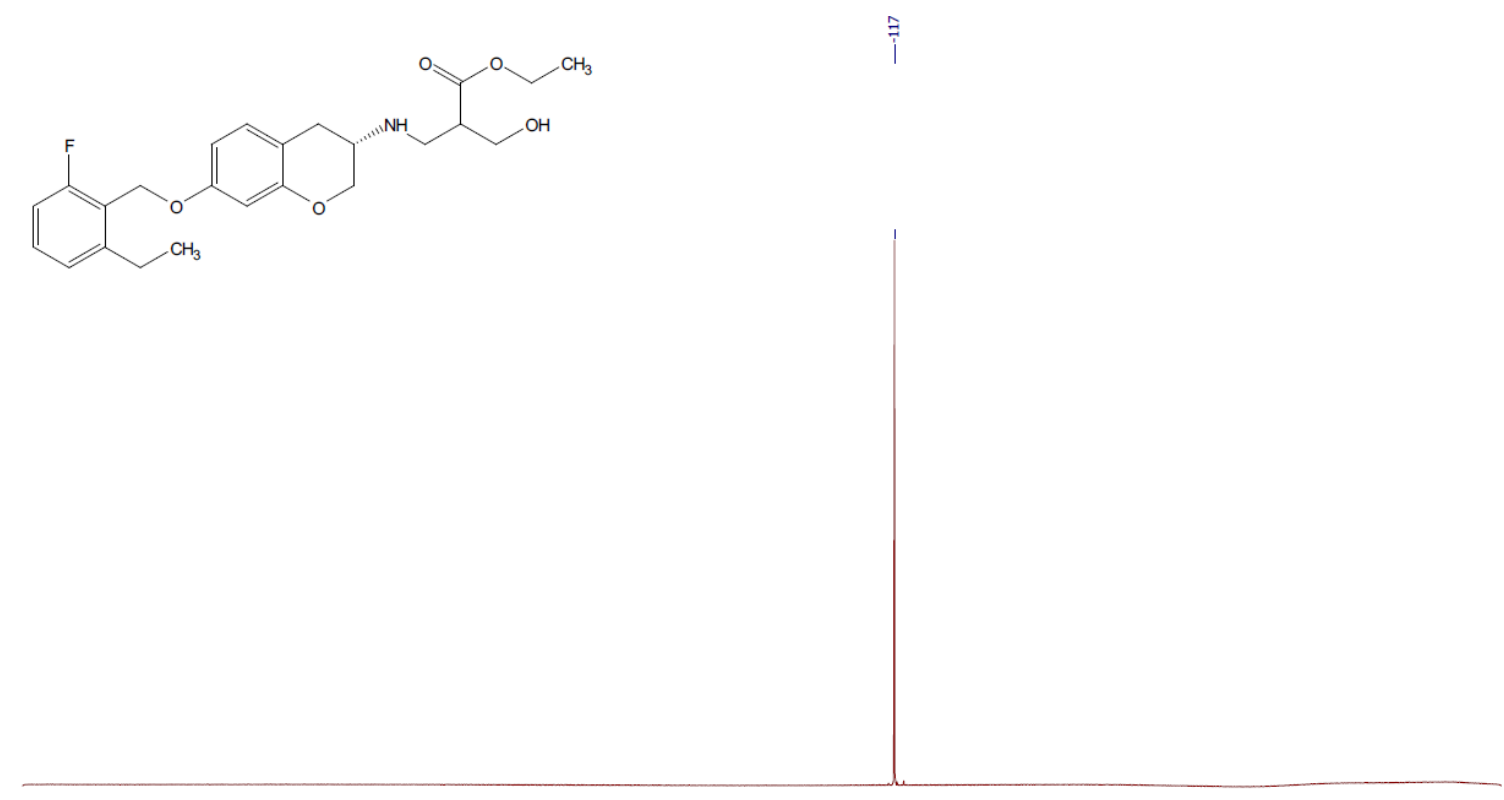

\begin{tabular}{|c|c|c|c|c|c|c|c|c|c|c|c|c|c|c|c|c|c|}
\hline & -20 & -30 & -40 & -50 & -60 & -70 & -80 & -90 & $f 1(\mathrm{nnm})$ & -110 & -120 & -130 & -140 & -150 & -160 & -170 & -180 \\
\hline
\end{tabular}

${ }^{19} \mathrm{~F}$ NMR spectrum of compound $57\left(471 \mathrm{MHz}, \mathrm{CDCl}_{3}, \mathrm{rt}\right)$. 


\section{References:}

(1) T. Ogata, J. Hartwig, J. Am. Chem. Soc. 2008, 130, 13848-13849.

(2) Y. Yoshida, Sh. Otsuka, K. Nogi, H. Yorimitsu, Org. Lett. 2018, 20, 1134-1137.

(3) A. Tili, F. Monnier, M. Taillefer, Chem. Comm. 2012, 48, 6408-6410.

(4) B. Fors, N. Davis, S. Buchwald, J. Am. Chem. Soc. 2009, 131, 5766-5768.

(5) W.-B. Sun, P.-Z. Zh., T. Jiang, Ch.-K. Li, L.-T. An, A. Shoberu, J.-P. Zou, Tetrahedron 2016, 72, 6477-6483.

(6) J. Jiang, H. Zhu, Y. Shen, T. Tu, Org. Chem. Front. 2014, 1, 1172-1175.

(7) A. Hajra, Y. Wei, N. Yoshikai, Org. Lett. 2012, 14, 5788-5491.

(8) Ch. Salom, P. Wagner, M. Bollenbach, F. Bihel, J.-J. Bourguignon, M. Schmitt, Tetrahedron 2014, 70, 3413-3421.

(9) M. Xiong, Z. Gao, X. Liang, P. Cai, H. Zhu, Y. Pan, Chem. Commun. 2018, 54, 9679-9682.

(10) C. Guan, Y. Feng, G. Zou, J. Tang, Tetrahedron 2017, 73, 6906-6913.

(11) M. O. Konev, T. A. McTeague, J. W. Johannes, ACS Catal. 2018, 8, 9120-9124.

(12) P. Wagner, M. Bollenbach, Ch. Doebelin, F. Bihel, J.-J. Bourguignon, Ch. Salomé, M. Schmitt, Green Chem. 2014, 16, 4170-4178.

(13) Ch. Desmarets, R. Schneider, Y. Fort, J. Org. Chem. 2002, 67, 3029-3036.

(14) A. Correa, C. Bolm, Angew. Chem. Int. Ed. 2007, 46, 8862-8865.

(15) W. Paisuwan, Th. Chantra, P. Rashatasakhon, M. Sukwattanasinitt, A. Ajavakom, Tetrahedron 2017, 73, 3363-3367.

(16) F. D. Huang, C. Xu, D. D. Lu, D. S. Shen, T. Li, F. S. Liu, J. Org. Chem. 2018, $83,9144-9155$

(17) T. N. Ansari, A. Taussat, A. H. Clark, M. Nachtegaal, S. Plummer, F. Gallou, S. Handa, ACS Catal. 2019, 9, 10389-10397.

(18) C. Gabriel, M. Keener, F. Gallou, B. Lipshutz, Org. Lett. 2015, 17, 3968-3971.

(19) C. Guissart, A. Barrros, L. R. Barata, G. Evano, Org. Lett. 2018, 17, 5098-5102. 